\title{
Studying climate signals through microscale chemical variability in diatoms
}

A high-resolution chemical imaging study of biogenic silica

Shaun Akse 

Utrecht Studies in Earth Sciences 206

\section{Studying climate signals through microscale chemical variability in diatoms.}

A high-resolution chemical imaging study of biogenic silica

Shaun Akse

Utrecht 2020 


\section{Members of the dissertation committee:}

Prof. dr. Raja Ganeshram

Dr. Kate Hendry

Prof. dr. Caroline Slomp

Prof.dr. Appy Sluijs

Prof. dr. George Swann
The University of Edinburgh, United Kingdom

University of Brisol, United Kingdom

Utrecht University, The Netherlands

Utrecht University, The Netherlands

University of Nottingham, United Kingdom

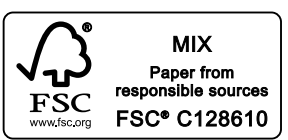

ISBN 978-90-6266-569-3

ISSN 2211-4335

USES No. 206

Author contact: shaun.akse@gmail.com

Cover: Diatom illustrations by Mae Akse. Design by Margot Stoete

Copyright (C) 2020 S.P. Akse. All rights reserved. No part of this publication may be reproduced in any form, by print or photo print, microfilm or any other means, without written permission by the publishers.

Printed in the Netherlands by Ipskamp 


\title{
Studying climate signals through microscale chemical variability in diatoms
}

\author{
A high-resolution chemical imaging study of biogenic silica \\ Het in beeld brengen van chemische variabiliteit in diatomeeën op microschaal \\ om het klimaat te bestuderen \\ (met een samenvatting in het Nederlands)
}

\begin{abstract}
Proefschrift
ter verkrijging van de graad van doctor aan de Universiteit Utrecht

op gezag van de rector magnificus, prof. dr. H.R.B.M. Kummeling, ingevolge het besluit van het college voor promoties in het openbaar te verdedigen op
\end{abstract}

vrijdag 28 februari 2020 des middags te 4.15 uur

door

Shaun Patrick Akse

geboren op 11 januari 1991 te Delft 


\section{Promotor:}

Prof. dr. J.J. Middelburg

\section{Copromotor:}

Dr. L. Polerecky

This thesis was accomplished with funding from the Netherlands Earth System Science Centre (NESSC), which is financially supported by the Ministry of Education, Culture and Science (OCW) of the Netherlands.

The NanoSIMS facility at Utrecht University was financed through a large infrastructure grant by the Netherlands Organisation for Scientific Research (NWO, grant no. 175.010.2009.011). 
"Few objects are more beautiful than the minute siliceous cases of diatomaceae: were these created that they might be examined and admired under the higher powers of the microscope?"

- Charles Darwin; On the Origin of Species by Means of Natural Selection. 1859 



\section{Contents}

\section{Chapter 1}

Introduction and synopsis $\quad 11$

1.1 The global silicon cycle 11

1.2 Biomineralization and structure of biogenic silica 12

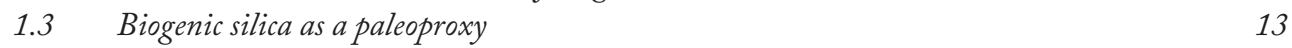

1.3.1 Isotopic signatures of biogenic silica $\quad 13$

1.3.2 Elemental ratios in diatom frustules $\quad 15$

1.4 The application of high-resolution imaging techniques to biogenic silica 17

1.5 Aim and outline of this research 20

\section{Chapter 2}

Assessing the proxy potential of diatom-bound Fe using high-resolution elemental imaging

2.1 Introduction 24

2.2 Materials E' Methods 25

2.2.1 Materials 25

2.2.2 Sample preparation and NanoSIMS imaging 26

$\begin{array}{ll}2.2 .3 & \text { SIMS analysis }\end{array}$

2.3 Results 27

2.3.1 Distribution of $\mathrm{Fe}$ and $\mathrm{Al}$ in cleaned fossil diatom frustules $\quad 27$

2.3.2 Analysis of diatom frustule interiors vs. exteriors $\quad 27$

2.3.3 SIMS measurements with the novel approach 29

2.4 Discussion 31

2.4.1 Aluminium contamination in fossil diatom frustules 31

2.4.2 Protective nature of the diatom frustule 31

2.4.3 SIMS measurements: applying the new approach 32

2.4.4 Implications and applications 33

2.5 Conclusion 34

Supplements $\quad 35$

\section{Chapter 3}

Mapping of organic signals in individual fossil diatom frustules with nanoSIMS and Raman spectroscopy $\quad 49$

3.1 Introduction 50

3.2 Materials E Methods 51

3.2.1 Materials 51 
3.2.2 Sample preparation and selection

3.2.3 NanoSIMS imaging 51

3.2.4 Raman spectroscopy and imaging

3.3 Results $\quad 52$

3.3.1 Identification of organics by Raman spectroscopy

3.3.2 NanoSIMS imaging of frustules 53

3.3.3 Mapping of organics by Raman spectroscopy 53

3.4 Discussion 56

3.4.1 Comparison of the nanoSIMS and Raman measurements 56

3.4.2 Implications $\quad 56$

3.5 Conclusions $\quad 57$

Supplementary figures $\quad 58$

\section{Chapter 4}

Rapid post-mortem oxygen isotope exchange in biogenic silica $\quad 61$

4.1 Introduction $\quad 62$

4.2 Materials E' Methods 64

4.2.1 Materials 64

4.2.2 Incubation experiments 64

4.2.3 NanoSIMS measurements - diatom frustules 65

4.2.4 NanoSIMS measurements - sponge spicules and crystalline silica 65

4.2.5 NanoSIMS data analysis 66

4.2.6 Raman spectroscopy $\quad 67$

4.3 Results 67

4.3.1 ${ }^{18} O$-enrichment in biogenic amorphous silica vs. crystalline silica $\quad 67$

4.3.2 Rapid post-mortem ${ }^{18} \mathrm{O}$-enrichment in diatom frustules 69

4.3.3 ${ }^{18} \mathrm{O}$-enrichment in sponge spicules $\quad 70$

4.3.4 Raman spectroscopy of amorphous and crystalline silica $\quad 71$

4.3.5 Influence of heating on the ${ }^{18} \mathrm{O}$ atom fraction of sponge spicules 73

$\begin{array}{lll}4.4 & \text { Discussion } & 74\end{array}$

4.4.1 Post-mortem oxygen exchange in amorphous biogenic silica 74

4.4.2 Oxygen exchange in crystalline silica 76

4.4.3 Distribution of ${ }^{18} \mathrm{O}$ in biogenic amorphous silica 76

4.4.4 Raman measurements on amorphous and crystalline silica 77

4.4.5 The potential sources of oxygen signal 78

4.5 Conclusion 79

Supplementary material $\quad 80$

\section{Chapter 5}

The influence of sediment diagenesis and aluminium-content on the secondary oxygen isotope exchange of diatom frustules $\quad 87$

5.1 Introduction 88

5.2 Materials \& Methods $\quad 89$

5.2.1 Materials $\quad 89$ 
5.2.2 Sediment incubation experiment 90

5.2.3 Incubation experiment with added dissolved Al 90

5.2.4 Sample preparation and NanoSIMS imaging 90

5.2.5 NanoSIMS data processing $\quad 91$

5.3 Results 92

5.3.1 Post-mortem ${ }^{18} \mathrm{O}$-enrichment of diatom frustules in the sediment 92

5.3.2. Presence and influence of clay and other aluminosilicate contaminants on fossil frustules 92

5.3.3 Influence of dissolved Al on ${ }^{18} \mathrm{O}$-enrichment in fresh frustules 95

$\begin{array}{lll}5.4 \text { Discussion } & 97\end{array}$

5.5 Conclusion 99

Supplementary figures 100

\section{Chapter 6}

The application of high-resolution imaging techniques to biogenic silica - Evaluation of sample preparation methods and analytical approaches 109

$\begin{array}{ll}6.1 \text { Introduction } & 110\end{array}$

6.2 Sample preparation 110

$\begin{array}{ll}\text { 6.2.1 Requirements for analysis } & 110\end{array}$

6.2.2 Upper water column and cultured samples $\quad 111$

6.2.3 Sediment and sponge samples 112

6.3 NanoSIMS data acquisition $\quad 113$

6.3.1 Elements and interferences 113

$\begin{array}{ll}\text { 6.3.2 Performance over time } & 114\end{array}$

$\begin{array}{lll}\text { 6.3.3 Standards in nanoSIMS } & 115\end{array}$

6.3.4 Primary ion implantation and pre-sputtering protocols 116

$\begin{array}{ll}\text { 6.4 NanoSIMS data processing } & 118\end{array}$

6.4.1 Avoiding edge effects 118

6.5 Which other methods are suitable for the analysis of diatom frustules and other forms of biogenic silica? $\quad 121$

6.5.1 Secondary Ion Mass Spectrometry (SIMS) 121

6.5.2 Raman micro-spectroscopy 121

6.5.3 Electron Probe Microanalyser 122

6.5.4 Transmission electron microscope (TEM) 122

References

$\begin{array}{ll}\text { Nederlandse Samenvatting (Summary in Dutch) } & 141\end{array}$

$\begin{array}{ll}\text { Aknowledgments (Dankwoord) } & 145\end{array}$

$\begin{array}{ll}\text { Curriculum Vitae } & 149\end{array}$ 


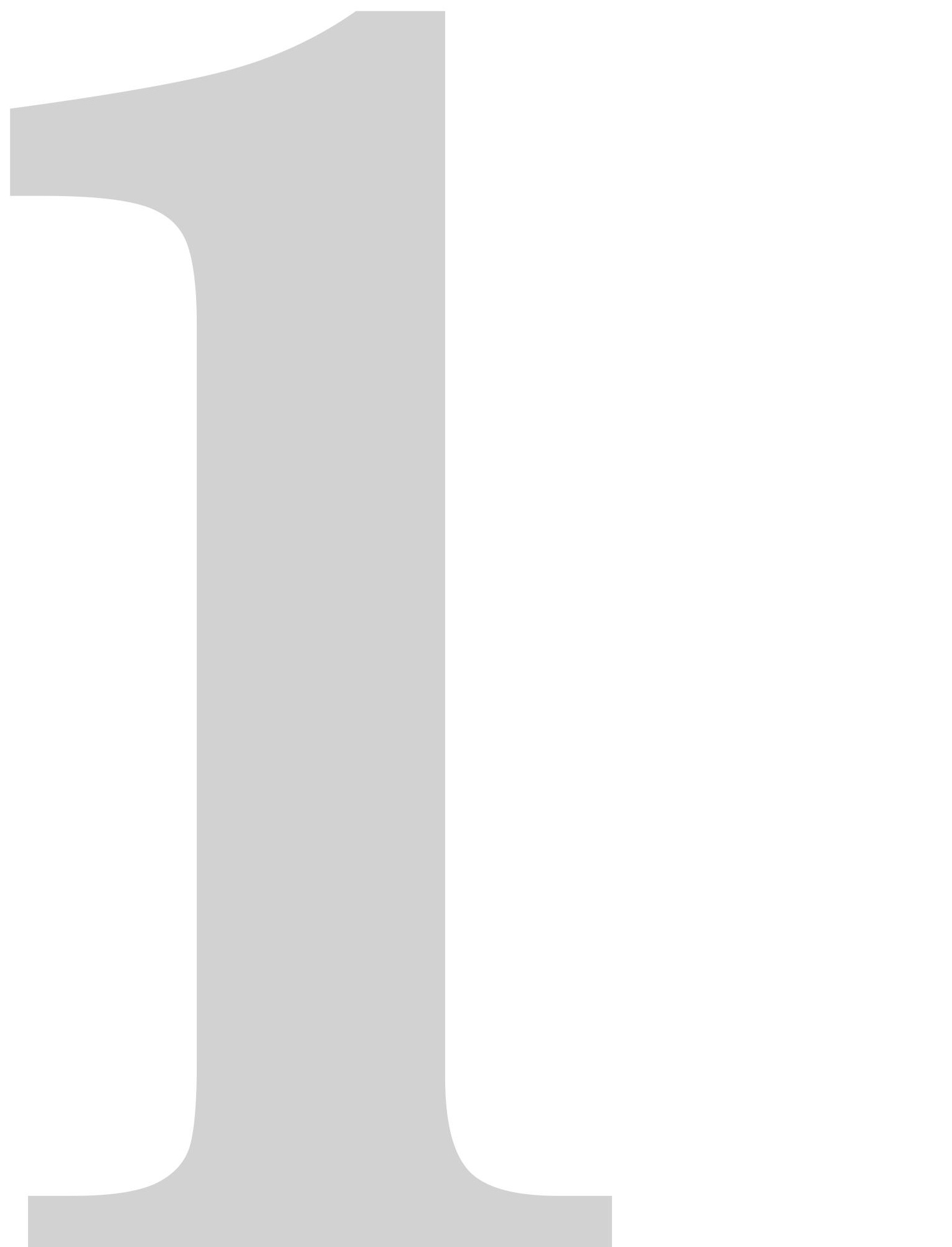




\section{Chapter 1}

\section{Introduction and synopsis}

\subsection{The global silicon cycle}

Silicon is the seventh-most-abundant element in the universe, and after oxygen the secondmost-abundant element in the Earth's crust. Furthermore, biogenic silica (opal) is, after calcium carbonate, the second most abundant mineral type formed by organisms (Brümmer, 2003). As such, the global silicon $(\mathrm{Si})$ cycle is an essential component of system Earth. It plays a critical role in maintaining climatic stability on geological timescales by forming an important sink of atmospheric $\mathrm{CO}_{2}$ through silicate weathering and carbon sequestration by diatoms (Walker, 1981; Berner et al., 1983; Siever, 1992; Ragueneau et al., 2000; Frings et al., 2016; Conley et al., 2017). Moreover, the organic carbon produced by siliceous phytoplankton acts as an important food resource and serves as a base for marine food webs (Sarthou et al., 2005; Armbrust, 2009).

Silicon pools exist in solid (crystalline \& amorphous) or dissolved forms in all reservoirs, from extra-terrestrial to continental to oceanic. In aqueous environments, silicon often occurs as dissolved silicic acid $\left(\mathrm{Si}(\mathrm{OH})_{4}\right)$ (Iler, 1973), an essential nutrient for the production of siliceous phytoplankton and other forms of biogenic silica (Ragueneau et al., 2000, and references therein). On geological timescales, the global oceanic Si cycle has evolved mainly due to the appearance and subsequent uptake of dissolved $\mathrm{Si}(\mathrm{dSi}$ ) for biomineralization of biogenic silica (Siever, 1992). The first drawdown of dSi occurred with the appearance of sponges and radiolaria in the Cambrian period (Conley et al., 2017) while diatoms did not appear until the Triassic (Sorhannus, 2007). The first diatoms in the fossil record appear in the Early Jurassic about 190 Myr ago (Sims et al., 2006 and references therein), but it is their rise to dominance in the Late Cretaceous (Damsté et al., 2004) that has led to almost ubiquitous depletion of $\mathrm{Si}(\mathrm{OH})_{4}$ observed in the surface and intermediate depths of the oceans today (Conley et al., 2017; Siever, 1992).

In modern oceans, the total gross biogenic silica $\left(\mathrm{bSiO}_{2}\right)$ production is estimated to be $240 \pm 40$

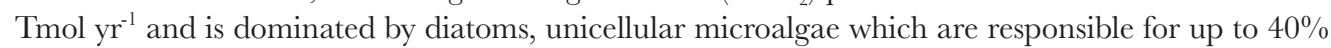
of marine primary production (Nelson et al., 1995). The contributions of other siliceous protists (such as silicoflagellates and radiolarians) and siliceous sponges are unknown but these likely play a more marginal role (Tréguer and De La Rocha, 2013).

Of the biogenic silica produced by diatoms, only about $5-7 \%$ is exported from the euphotic zone and ultimately buried in sediments, because the majority is recycled in the water column (Tréguer et al., 1995). As seen in Fig. 1.1, burial of diatoms forms the largest $\mathrm{bSiO}_{2}$ sink $(6.3 \pm 3.6$ Tmol Si yr ${ }^{-1}$; Tréguer and De La Rocha, 2013) followed by the burial of sponges $(1.7 \pm 1.6 \mathrm{Tmol}$ 
Si $\mathrm{yr}^{-1}$; Maldonado et al., 2019) and radiolaria (0.09 $\pm 0.05 \mathrm{TmolSiyr}^{-1}$; Maldonado et al., 2019). As efficient exporters of organic matter from the surface oceans, the recycling of $\mathrm{bSiO}_{2}$ directly influences the efficiency of the biological $\mathrm{CO}_{2}$ pump; hence atmospheric carbon dioxide.

\subsection{Biomineralization and structure of biogenic silica}

Solid-phase biogenic silica structures are created by several aquatic species such as diatoms, radiolaria, silicoflagellates and sponges, but also by continental species such as higher plants (Tréguer and De La Rocha, 2013; Conley, 2002). Biogenic silica is composed of amorphous silica, a random network mainly composed of interlinked $\mathrm{SiO}_{4}$ tetrahedral units connected through covalent Si-O-Si bonds of variable bond angle and bond lengths (Mann and Perry, 1986; Sutton et al., 2018). This structure forms via condensation reactions where two Si-OH groups of the silicic acid react to form $\mathrm{SiO}_{2}+\mathrm{H}_{2} \mathrm{O}$, which subsequently precipitates as solid $\mathrm{SiO}_{2}$. All the aforementioned organisms form amorphous silica by utilization of silicic acid, but the exact biomineralization processes involved in this differ per groups and species.

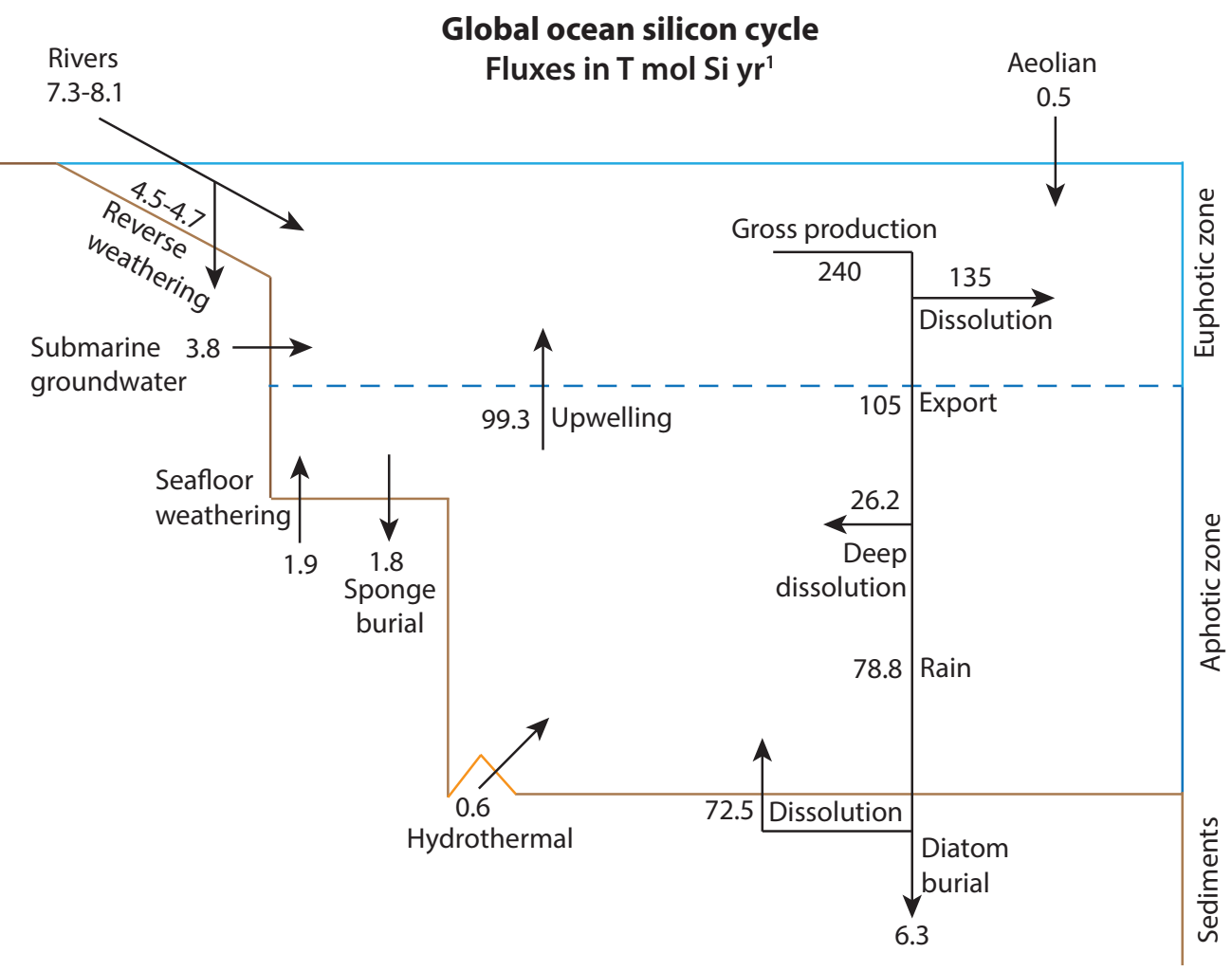

Figure 1.1: The global silicon cycle based primarily on Tréguer and De La Rocha, 2013; Rahman et al., 2017; Maldonado et al., 2019; Rahman et al., 2019 
Diatoms, for example, are a predominantly planktic species that are represented in the sediment by their siliceous housing termed frustule. Diatoms form their frustules by creating an intracellular supersaturated solution of silicic acid (silicon deposition vesicle), supplied through a passive diffusion across the cell membrane, and depositing the newly formed biosilica structure on an organic template (Thamatrakoln and Hildebrand, 2008; Hildebrand et al., 2018). Alternatively, siliceous sponges are benthic species and are commonly represented in the sediments by spicules, the structural elements which support the skeleton of the animal. For sponge spicules, the precipitation of solid silicate is governed by an enzyme termed silicatein (Cha et al., 1999). This enzyme forms an organic filament, called an axial filament, around which concentric layers of hydrated silica are deposited (Uriz et al., 2003; Uriz, 2006).

\subsection{Biogenic silica as a paleoproxy}

Early proxy research concerning biogenic silica focused on fossil diatom frustule species assemblages. Specifically, in the Southern Ocean these changes were of great importance in studying, for example, glacial-interglacial transitions (e.g., Crosta et al., 2004). In the last few decades, the chemical signature of fossil biogenic silica has been receiving increased attention. This increased interest can be linked to the scarcity or complete absence of carbonates, a traditional target material for paleoproxy research, in sections of the world's sediment records (particularly in high-latitude regions). Fortunately, these records are often dominated by silica microfossils. As diatom frustules are often an order of magnitude smaller than popular calcareous microfossils such as foraminiferal tests, the increased interest in frustule chemistry can also be linked to the development of high spatial resolution analytical techniques. Overall, a variety of geochemical tools have been developed looking at changes in isotopic signatures, elemental ratios and organic biomarkers. Proxies relevant to this thesis will be summarized in the next section while comprehensive overviews of the different diatom proxies can be found in Leng \& Swann (2010) and Sutton et al. (2018).

\subsubsection{Isotopic signatures of biogenic silica}

\section{Oxygen isotopes}

The oxygen isotope composition of diatom frustules has been the subject of study because it is thought that, similar to the $\delta^{18} \mathrm{O}_{\text {foraminifera, }}$ the $\delta^{18} \mathrm{O}_{\text {diatom }}$ reflects the isotopic composition of the ambient seawater. Degens and Epstein (1962) were the first to expand the $\delta^{18} \mathrm{O}$ analytical techniques to silica, by measuring cherts and diatomites. Since then this type of analysis has been under steady development and has been applied to several diatom records (e.g. Shemesh et al., 1995; Swann et al., 2006) The $\delta^{18} \mathrm{O}$ of water is, among others, a reflection of changes in global ice volume, as well as local changes in evaporation, freshwater inputs and thus salinity, and temperature. Different from the $\delta^{18} \mathrm{O}$ of foraminifera, it is generally assumed that the $\delta^{18} \mathrm{O}$ of diatom frustules found in the sediment, is representative only of $\delta^{18} \mathrm{O}$ changes in the euphotic zone of the water column due to the need for light by diatoms to photosynthesize (Swann and Leng, 2009).

Other forms of biogenic silica are not commonly used for $\delta^{18} \mathrm{O}$ analysis. Radiolaria have been shown to undergo extensive isotopic reequilibration (Matheney and Knauth, 1989) and studies have suggested that the fractionation between sponge spicules and seawater is not systematically reliable, despite some studies showing clear trends in $\delta^{18} \mathrm{O}$ (Matheney and Knauth, 1989; Matteuzzo et al., 
2013; Snelling et al., 2014). Instead, the study of sponge spicules as a paleoproxy has focused on silicon isotope studies (e.g. Hendry et al., 2010; Hendry and Robinson, 2012).

Despite a continued development, there are still significant concerns surrounding the $\delta^{18} \mathrm{O}_{\text {diatom }}$ proxy. These concerns arise around the uncertainty associated with the calculated silica-water fraction factors (Fresh/recent: Brandriss et al., 1998; Dodd and Sharp, 2010; Sedimentary: e.g., Juillet-Leclerc and Labeyrie, 1987; Matheney and Knauth, 1989; Shemesh et al., 1992). The hydrous ( $\mathrm{Si}-\mathrm{OH}$ ) layer of the frustule has been identified as a notable problem for the analysis of $\delta$ ${ }^{18} \mathrm{O}$ in diatoms. The oxygen in the silanol groups $(\mathrm{Si}-\mathrm{OH})$ are thought to be in continual exchange with any water the frustule is exposed to both during and after sedimentation. As such the hydrous layer will reflect a combination of signals from surface water, sediment pore water, laboratory water and atmospheric moisture (Tyler et al., 2017). Studies indicate that between 7-40\% of all oxygen in a diatom frustule may originate post-depositionally (Swann and Leng, 2009 and reference therein). Several analytical techniques have been developed to reduce the role of exchangeable oxygen in silanol groups in the measured $\delta^{18} \mathrm{O}$ which are reviewed in Chapligin et al. (2011). However, despite these efforts, there is still much uncertainty surrounding the potential of post-mortem oxygen exchange on geological timescales (Dodd et al., 2017).

\section{Silicon isotopes}

The temporal and spatial variability in the silicon isotope composition of diatom frustules has been used for paleoreconstructions (De La Rocha et al., 1997; Basile-Doelsch et al., 2005; Brzezinski et al., 2002; Leng et al., 2009). Diatoms fractionate silicon isotopes during growth uptake (preferring the incorporation of lighter isotopes), allowing the possibility to trace both the modern marine biogeochemical cycle of silicon as well as potential changes in the past (De La Rocha et al., 1997). Due to the close link of the $\mathrm{Si}$ and $\mathrm{C}$ cycles, information obtained from the $\delta^{30} \mathrm{Si}_{\text {diatom }}$ marine records can help study the role of the biological pump in the drawdown and transport of carbon from the atmosphere to the deep ocean (Brzezinski et al., 2002; Matsumoto et al., 2002). The study of $\delta^{30} \mathrm{Si}$ in siliceous sponge spicules was first discussed in De la Rocha (2003) and has since been developed further by several other studies (e.g. Hendry et al., 2011; Wille et al., 2010). It has been suggested that $\delta^{30} \mathrm{Si}$ of spicules is not affected by environmental factors other than $\mathrm{Si}(\mathrm{OH})_{4}$ concentrations and therefore represents a robust proxy for changes in past bottom-water $\mathrm{Si}(\mathrm{OH})_{4}$ levels (Hendry and Robinson, 2012). By combining this with surface water information provided by $\delta^{30} \mathrm{Si}$ of diatoms, this is a promising tool to reconstruct silicon levels and surface-to-bottom water silicon gradients in the past.

A common issue with isotope analysis of solid-phase records is the impact that dissolution (the removal of lighter isotopes) and potential reprecipitation can have on the acquired signal. Multiple studies have looked at this; Demarest et al. (2009), for example, found a small silicon isotope fractionation during dissolution of diatom opal collected from surface waters and sediment traps ( 1000m depth), whereas Wetzel et al. (2014) and Ehlert et al., 2016 present results that indicate that silicon isotope fractionation during dissolution of sediment core diatoms is absent. It is suggested that fractionation during dissolution is controlled by the reactivity of the frustule, and potentially the aluminum content. As reactivity may vary temporally and spatially, this should be kept in mind when interpreting $\delta^{30} \mathrm{Si}$ data. And while a constant fractionation factor is assumed for the interpretation of $\delta^{30} \mathrm{Si}$ for dSi utilization, this has recently been challenged by a study showing species-dependent $\delta^{30}$ Si fractionation variability (Sutton et al., 2013). Furthermore, a study looking at secondary exchange and fractionation between aqueous and amorphous Si found that about $50-85 \%$ isotope exchange was achieved within $\sim 60$ days at room temperature (Zheng et al., 2019). 
Overall, additional research is needed to constrain the environmental, species and diagenetic effects on $\delta^{30} \mathrm{Si}$ of diatom frustules.

\section{Nitrogen and carbon isotopes}

The analysis of $\delta^{13} \mathrm{C}$ and $\delta^{15} \mathrm{~N}$ in diatom frustules is based on the presence of organic matter entombed in the silica structure (Sigman et al., 1999; De La Rocha, 2006). The organic matter from which these isotopes can be measured is thought to originate from several proteins that are necessary for construction of the silica frustule itself (Kröger et al., 2002; Hecky et al., 1973; Kröger and Poulsen, 2008; Bridoux and Ingalls, 2010). However, as of yet, it is unknown where exactly in the frustule this organic matter resides. Despite this, research suggests that frustule-bound $\mathrm{N}$ and $\mathrm{C}$ are protected from diagenesis. This assumption is based on indirect observations such as the similar relative amino acid abundances in the organic matter of fossil frustules and living diatoms (Shemesh et al., 1993); the apparent absence of diagenetic variability in frustule-bound N when compared to bulk sediment N content (Sigman et al., 1999); and high diatom-bound amino acid concentrations in silica rich sediments relative to sediment traps (Ingalls et al., 2003).

The $\delta^{15} \mathrm{~N}_{\text {diatom }}$ proxy has been used to reconstruct nitrate utilization in surface waters (Sigman et al., 1999; Brzezinski et al., 2002; Robinson et al., 2004; Schneider-Mor et al., 2005; Des Combes et al., 2008). During a period of growth, phytoplankton draw down nutrient concentrations, resulting in an increase of residual nitrate $\delta^{15} \mathrm{~N}$ due to fractionation of $\mathrm{N}$ isotopes during nutrient uptake (through Rayleigh distillation). This rise in nitrate $\delta^{15} \mathrm{~N}$ with nutrient depletion should be recorded in the opal produced by diatoms (De La Rocha, 2006). As such, high $\delta^{15} \mathrm{~N}_{\text {diatom }}$ indicate that a greater fraction of available nutrients (nitrate) has been consumed during the growing season (Shemesh et al., 1993). The $\delta^{13} \mathrm{C}$ of diatom frustules is seen as a proxy for $\mathrm{CO}_{2}$ (aq) concentration and primary production (Singer and Shemesh, 1995; Schneider-Mor et al., 2005; Des Combes et al., 2008; Rosenthal et al, 2000; Stoll et al., 2016). The signal is predominantly controlled by the balance between supply and demand for Dissolved Inorganic Carbon (DIC), which in turn is driven by changes in biological productivity and cellular carbon concentrations. In addition to the uncertainty surrounding the distribution of organic signals in the frustules, an uncertainty in the $\delta^{15} \mathrm{~N}$ proxy comes from variability observed in the magnitude of nitrogen isotopic discrimination by diatoms. These changes may be attributed to several factors including light levels, growth rates and possible species-specific vital effects (Karsh et al., 2003; Needoba et al., 2003; Needoba and Harrison, 2004; Des Combes et al., 2008). Similarly, factors such as cell growth rate, size and shape as well biochemical metabolic pathways can affect $\delta^{13} \mathrm{C}_{\text {diatom }}$ (Popp et al., 1998; Schneider-Mor et al., 2005).

\subsubsection{Elemental ratios in diatom frustules}

Diatoms frustules contain various trace elements which may be utilized for paleoproxies. This thesis focuses on $\mathrm{Fe}$ and $\mathrm{Al}$.

\section{$\mathrm{Fe} / \mathrm{Si}$}

A direct correlation between dissolved $\mathrm{Fe}(\mathrm{dFe})$ concentrations in surface sea water and frustule $\mathrm{Fe} /$ Si has been observed, leading to the introduction of frustule Fe/Si ratios as a novel proxy to track iron availability during diatom growth (Pichevin et al., 2014). Frustule-bound iron concentrations in a sediment core were reported from an upwelling region (the Gulf of California) and used to link dissolved Fe in surface water with sediment silica burial. By coupling the Fe/Si measurements to other established diatom-bound geochemical tracers such as silicon isotopes and the bulk 
biogenic silica $\left(\mathrm{wt}^{\mathrm{o}} \%\right)$, a more comprehensive picture can be obtained on the role of iron availability in upwelling regions. Understanding changes in iron-availability is of interest because Fe is a key nutrient for diatom growth and is closely associated with the presence of other macronutrients such as $\mathrm{N}$ and $\mathrm{P}$ as well as $\mathrm{Si}(\mathrm{OH})_{4}$. For example, Fe-replete growth conditions can lead to more heavily silicified diatom frustules and therefore greater Si burial (Hutchins and Bruland, 1998; Takeda, 1998). Since changes in diatom frustule silicification and $\mathrm{Si}$ burial have been shown to impact the oceanic carbon cycle via changes in the transport of organic carbon (Arellano-Torres et al., 2011; Pichevin et al., 2012) the study of $\mathrm{Fe} / \mathrm{Si}$ in frustules may give insights into $\mathrm{CO}_{2}$ uptake changes in past upwelling regions. While it has been observed that Fe is structurally incorporated into the frustule during diatom growth (Ingall et al., 2013), frustules may also obtain additional Fe during post-mortem diagenetic processes. An important marker to check for potential diagenesis is the concentration of $\mathrm{Al}$ present in the frustule.

$\mathrm{Al} / \mathrm{Si}$

$\mathrm{Al} / \mathrm{Si}$ ratios have often been associated with post-depositional alteration of frustules. Fossil diatoms often have relatively high $\mathrm{Al}$ levels, as several studies have suggested that structural incorporation of $\mathrm{Al}(\mathrm{III})$ reduces the solubility of biogenic silica (e.g. van Bennekom et al., 1989), improving the likeliness of preservation. Diatoms can also take up aluminium during frustule biosynthesis, but studies have shown that the $\mathrm{Al}$ content of the frustule does not exceed $0.8 \%$, even when grown at high Al concentrations (Van Beusekom et al., 1989; Vrieling et al., 1999; Koning et al., 2007). Interestingly, studies have shown that $\mathrm{Al} / \mathrm{Si}$ ratios of open ocean plankton assemblages are at least an order of magnitude lower than those found in diatoms isolated from sediments (van Bennekom et al., 1989; Dixit et al., 2001; Koning et al., 2002; Koning et al., 2007). Therefore high Al/Si ratios in diatom frustules from the sediments would suggest post mortem incorporation of $\mathrm{Al}$. $\mathrm{Al}$ can be taken up quickly at the sediment-water interface by either forming an aluminosilicate coating or through structural incorporation (Cappellen and Qiu, 1997; Dixit et al., 2001; Koning et al., 2007; Ren et al., 2013). It is argued in Ren et al. (2013), that the aluminium incorporated secondarily into the diatom frustules is primarily released from the dissolution of lithogenic minerals in the sediments. It has further been shown that the formation of cation-rich aluminosilicates on biogenic silica, due to reverse weathering, can occur on relatively short timescales $(<1 \mathrm{yr})$ (Michalopoulos and Aller, 2004; Michalopoulos et al., 2000). Taken together, Al/Si may not form a traditional proxy but may instead be used as a marker of preservation and diagenesis.

\section{Other trace elements}

Biogenic silica can host a wide array of different trace elements in the silica matrix, many of which have received much attention for their paleoproxy potential. Germanium, for example, mimics the behaviour of silica and may thus be incorporated into the frustule matrix of diatoms (Azam et al., 1973; Shemesh et al., 1988). Furthermore, seawater germanium is derived predominantly from weathering of silicate minerals and is effectively removed from the surface ocean by diatoms and deposited into the sediment (Bareille et al., 1998; Tréguer et al., 1995; Froelich et al., 1985). The $\mathrm{Ge} / \mathrm{Si}$ in opal should thus reflect changes in relative rates of continental weathering (Shemesh et al., 1988; Froelich et al., 1992).

The uptake of $\mathrm{Zn}$ by diatoms has also been a point of interest as a strong coupling of the $\mathrm{Zn}$ and Si nutrient profiles is observed in the oceans (Bruland et al., 1978). Even though the majority of $\mathrm{Zn}$ in diatoms $(>97 \%)$ is hosted in the organic matter (Ellwood and Hunter, 2000; Twining and Baines, 2013) the $\mathrm{Zn}$ in opal is thought to reflect $\mathrm{Zn}^{2+}$ availability (Andersen et al., 2011). This 
proxy has been applied, for example, for changes in Zn supply to surface waters due to changes in mixed-layer salinity (or freshwater input) in coastal Antarctica (Hendry and Rickaby, 2008).

Other trace elements in frustules that have received attention include: B (Mejía et al., 2013); Ag (Zhang et al., 2004; Chang et al., 2015) and Ni (Twining and Baines, 2013)

\subsection{The application of high-resolution imaging techniques to biogenic silica}

The size of most diatom frustules (micrometer scale) can complicate measurements with the traditional bulk techniques, such as inductively coupled plasma mass spectrometry (ICP-MS), as relatively large amounts of sample are necessary. This can necessitate the use of sediment size fractions, which can increase the amount of impurities present in the sample and thus lead to uncertainties. Clay mineral particles, for example, are extremely difficult to remove and complicate the structural and compositional characterization of diatom frustules (Gehlen et al., 2002). Being able to differentiate between the original metal content of the frustule, and secondary signals originating from diagenesis and contamination is an essential development needed for the advancement of the use of elemental ratios in biogenic silica. Similarly, for determining the isotopic composition of diatom silica, samples are required that consist of almost pure diatomite as the analytical techniques used (i.e. Fluorination methods and Isotope ratio mass spectrometry) will also liberate any contaminants (Leng and Swann, 2010). Studies have shown that even small contributions of contaminants can have significant influences on the isotope values (Brewer et al., 2008). Determining what factors influence the produced signals is therefore an important step forwards for the study of changing isotopic compositions in biogenic silica.

In modern laboratories there is an increasing array of potential techniques that may be applicable for diatom frustule analysis. Progress has been made with increasingly higher spatial resolution. For example, on a cell level, the localization and distribution of elements has been studied with synchrotron-based X-ray fluorescence (SXRF), in order to provide insights into cell chemistry (Twining et al., 2003). Raman spectroscopy has been applied to fresh diatom detritus (Kammer et al., 2010; De Tommasi et al., 2017). However, most studies focus on creating spectra while the imaging capabilities of the Raman have not been utilized as often. High-resolution studies of fossil frustules are less common but have been attempted. The Secondary Ion Mass Spectrometer (SIMS), for example, has been applied to cleaned fossil diatom frustules originating from a sediment in an attempt to exclude potential unwanted influences from clay particles (Pichevin et al., 2014). In another study, high-resolution imaging was performed using transmission electron microscopy (TEM) and scanning electron microscopy (SEM) combined with energydispersive X-ray spectroscopy (EDS) mapping analysis (Yuan et al., 2019), this technique allowed the study of the distribution and sources of minor elements in the frustule structure. The study by Yuan et al., (2019) is an example of the potential that high-resolution imaging techniques have for the application to fossil biogenic silica. In this thesis, we utilize this potential to improve existing paleoproxies using SIMS, nanoSIMS and Raman spectroscopy.

The SIMS and nanoSIMS employ the same basic principles but the nanoSIMS has markedly improved spatial resolution due to adjustments to the beam column and the source. The nanoSIMS employs two types of primary beams where the Cs+ source is used for negative ion analysis and Osource is used for positive ion analysis. Under ideal circumstances, beam sizes down to $\sim 50 \mathrm{~nm}$ can be achieved with a $\mathrm{Cs}^{+}$source and $\sim 150 \mathrm{~nm}$ with a $\mathrm{O}^{-}$duoplasmatron source (Kilburn and Wacey, 2014). 


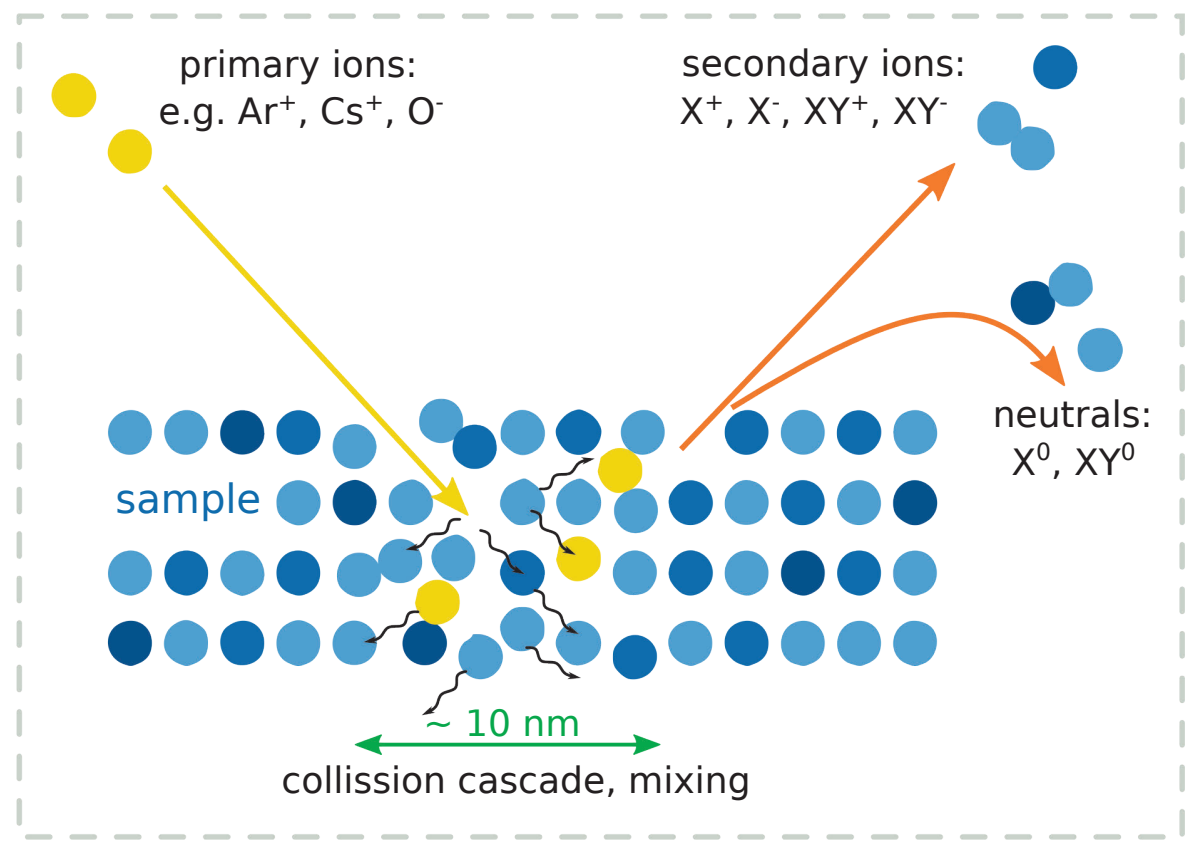

Figure 1.2: Principle of SIMS (Used with permission from Roepert, 2019) - The basic principles of the SIMS technique can be summarized as follows: a primary beam of energized ions is focused on a sample surface. The impact of these primary ions removes the top few atomic layers of sample material in a process referred to as sputtering. The released material consists of neutral and ionized atoms and molecules of which the ionized species are diverted by ion optics to the mass spectrometer where they are separated based on their mass-to-charge ratio and measured.

To achieve high spatial resolution, a significant reduction in beam current is needed compared to the traditional SIMS measurement (nA range), going down to $0.5 \mathrm{pA}$ in many of the measurements presented in this thesis. So, while this results in considerably better spatial resolution than the SIMS, this also implies significantly longer measuring time to obtain sufficient secondary ion counts.

In this thesis, the Raman technique has proven to be a strong complementary technique to the high-resolution imaging capabilities of the nanoSIMS. Raman spectroscopy is a relatively nondestructive technique that enables the study of the molecular composition of the target through the measurement of the vibrational modes of molecules. By being able to structurally fingerprint the silica samples, we gain additional insights into the nature of the chemical patterns we observe with the nanoSIMS. Furthermore, where topography changes are a cause of concern in nanoSIMS analyses (see Chapter 6), the Raman technique is less sensitive to this. 


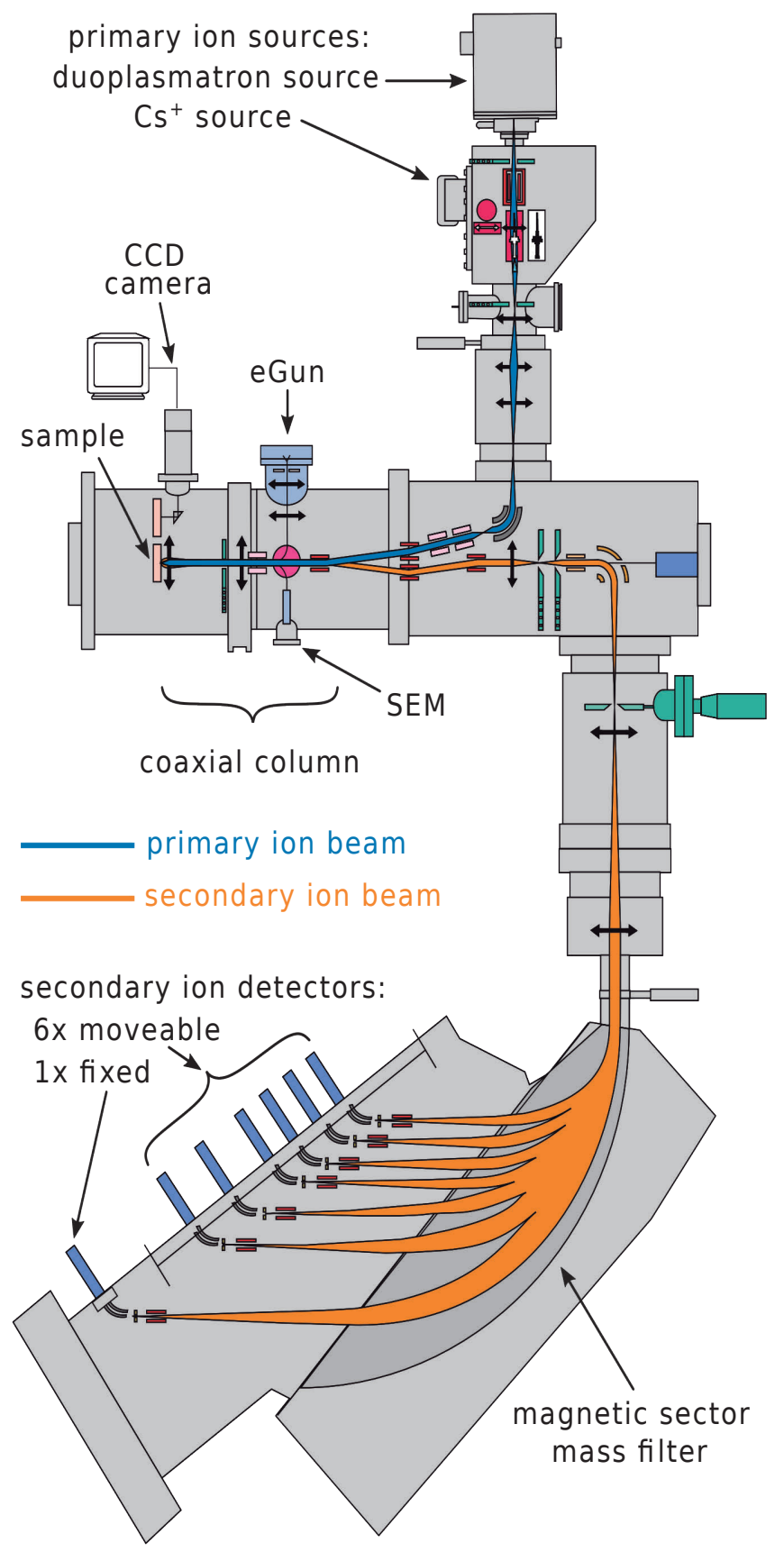

Figure 1.3: Schematic of the nanoSIMS 50L instrument used with permission from Cameca 


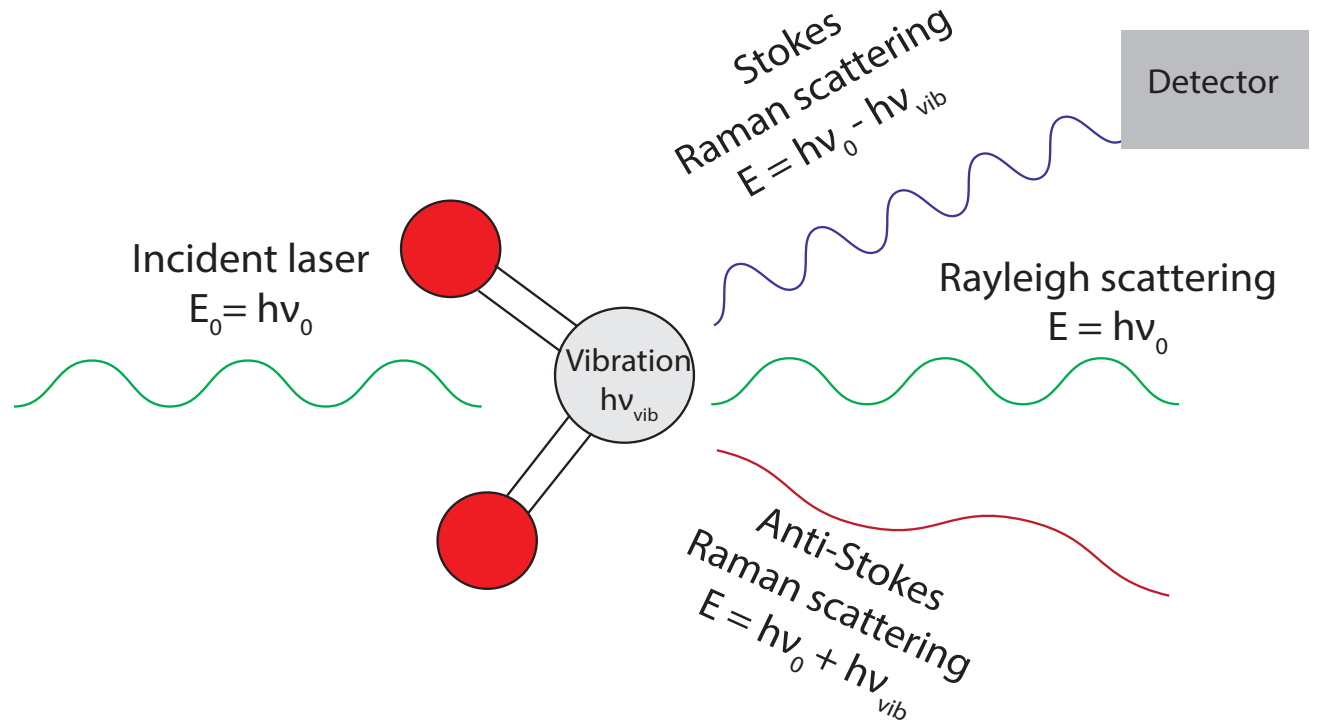

Figure 1.4: Principle of Raman spectroscopy Photons from a narrow-bandwidth light source (e.g., a high intensity laser) interact with a molecule. The majority of the photons are scattered elastically, i.e., without changing their energy and thus their wavelength (Rayleigh scattering). Due to the non-linear character of the interaction, however, the energy of a small fraction of photons $(0.0000001 \%)$ is increased or decreased by the amount corresponding to the vibrational energies of the molecule (Raman scattering). Measurement of the scattered light by the Raman spectrometer thus allows identification of chemical bonds between atoms in a molecule.

\subsection{Aim and outline of this research}

The aim of this research is to apply high-resolution chemical imaging techniques in an attempt to better understand and thus improve existing paleoproxies based on biogenic silica.

In Chapter 2 we study fossil diatom frustules with the aim to distinguish the original Fe-content, biologically incorporated into the frustule during diatom growth, from secondary $\mathrm{Fe}$ originating from terrigenous contamination and diagenesis. The Fe/ $\mathrm{Si}$ proxy has the potential to provide information on Fe-availability in past upwelling regions, and indirectly on Si burial and $\mathrm{CO}_{2}$ uptake. However, concerns linked to post-mortem diagenesis of diatom frustules, hampered the advancement of this proxy. By applying the nanoSIMS technique we were able to study the distribution of $\mathrm{Fe}$ and $\mathrm{Al}$ (a marker for diagenesis levels) in cleaned fossil diatom frustules. Despite the application of a well-established cleaning technique, we observed the presence of $\mathrm{Al}$ hotspots that co-localized with stronger Fe signals. However, it was also observed that the interior of the frustule had generally lower $\mathrm{Al} / \mathrm{Si}$ values, suggesting reduced diagenetic influences. Based on these results, sample preparation and measuring protocols for $\mathrm{Fe} / \mathrm{Si}$ determination with SIMS were adjusted by (i) including an additional cleaning step and (ii) targeting of frustule interiors. These adjustments lowered the $\mathrm{Al} / \mathrm{Si}$ and $\mathrm{Fe} / \mathrm{Si}$ of measured frustules significantly, and should therefore lessen the concerns surrounding diagenetic overprinting of diatom frustules and hence result in a more robust paleoproxy.

In Chapter 3 we aim to image the presence and distribution of organic signals in thoroughly cleaned fossil diatom frustules. The application of $\delta^{15} \mathrm{~N}_{\text {diatom }}$ is based on the assumption that the 
organic matter occluded in the silica is protected from diagenesis. However, the location of the organic matter within the frustule has hitherto not been identified. By combining high spatial resolution imaging by nanoSIMS (surface technique) and Raman micro-spectroscopy (larger depth of probing), we were able to, for the first time, image the presence and distribution of the organics tightly bound to the silica matrix. The nanoSIMS images revealed that the organic signals were present throughout, but localized concentrations were observed around the pores. Raman measurements confirmed the heterogenous presence of organics but could not resolve the spatial patterns observed by nanoSIMS.

Chapter 4 looks at diagenetic overprinting of the $\delta^{18} \mathrm{O}$ signal in various forms of silica. Both biogenic amorphous silica (diatom frustules and siliceous sponge spicules) and crystalline silica samples were incubated in ${ }^{18} \mathrm{O}$-enriched seawater for various time intervals. The nanoSIMS results show that, on timescales similar to that of the sinking diatom detritus in marine systems (days to weeks), the $\delta^{18} \mathrm{O}$ signal of both diatom frustules and sponge spicules can be homogenously overprinted. In contrast, crystalline silica does not show any presence of ${ }^{18} \mathrm{O}$-enrichment, most likely due to the absence of silanol (Si-OH) groups as observed in Raman measurements. Together these results confirm that the $\mathrm{Si}-\mathrm{OH}$ groups can be diagenetically overprinted, and suggest that the rest of the silica structure is also susceptible. The final measured $\delta^{18} \mathrm{O}_{\mathrm{bSiO} 2}$ will most likely represent a mixed signal from the original growing stage, water-column settling, sediment-water interface, and sediment pore waters.

Chapter 5 continues our research on secondary oxygen overprinting but shifts the focus to diagenetic processes in the sediment. After our results from Chapter 4 showed that diagenesis of $\delta^{18} \mathrm{O}_{\text {diatom }}$ already occurs in the water column, the question arose whether this would continue in the sediment or if this would be inhibited by additional processes. To resolve this, two experiments were set up. The first ${ }^{18} \mathrm{O}$-incubation experiment put packages of fresh diatom detritus in a diatomaceous clay and at the sediment-water interface. From this experiment we could compare different phases of diagenesis in the sediment. NanoSIMS analysis showed that the fresh diatom detritus and fossils frustules underwent significant ${ }^{18} \mathrm{O}$-enrichment. The degree to which this occurred varied; the fresh frustules placed at the sediment-water interface were most enriched followed by the fresh diatoms buried in the sediment and finally the fossil frustules. Furthermore, we observed that surface $\mathrm{Al}$ deposits on the fossil frustules did not inhibit the ${ }^{18} \mathrm{O}$-enrichment of the silica underneath. In a second experiment, varying concentrations of dissolved $\mathrm{Al}(\mathrm{dAl})$ were added to ${ }^{18} \mathrm{O}$-enriched seawater. The increase of dAl led to an increase of the Al-content of the frustules. The distribution of the additional $\mathrm{Al}$ appeared to be homogenous throughout the frustule valve, suggesting an incorporation mechanism where $\mathrm{Al}$ replaces $\mathrm{Si}$ in the matrix. The addition of $\mathrm{Al}$ significantly lowered the amount of ${ }^{18} \mathrm{O}$-enrichment. These results led us to hypothesize that, although the incorporation of $\mathrm{Al}$ can slow the exchange of secondary oxygen, it likely continues to some degree on geological timescales. This confirms our conclusion from Chapter 4 that the $\delta^{18} \mathrm{O}$ signal measured in fossil diatom frustules likely reflects a combination of signals originating from the growth phase to the phase where the frustules interact with the porewaters.

In Chapter 6, I provide an overview of my experiences with the nanoSIMS and other highresolution imaging techniques gained during my work with biogenic silica. There is very little precedent concerning the application of the nanoSIMS to biogenic silica. This chapter showcases the many different approaches that were attempted to best utilize the nanoSIMS for biogenic silica. Both the successful and unsuccessful attempts are evaluated, which will hopefully aid future researchers interested in unravelling the many mysteries still surrounding the chemistry of diatom frustules. 


$$
2
$$




\title{
Chapter 2
}

\section{Assessing the proxy potential of diatom-bound Fe using high-resolution elemental imaging}

\author{
Shaun P. Akse', Laetitia E. Pichevin ${ }^{2}$, Lubos Polerecky ${ }^{1}$ and Jack J. Middelburg ${ }^{1}$ \\ Department of Earth Sciences, Utrecht University, PO Box 80021, 3508 TA Utrecht, The Netherlands \\ 2 School of Geosciences, Grant Institute, University of Edinburgh, West Main Road, Edinburgh EH9 37W, UK
}

\begin{abstract}
The Fe/Si ratio in fossil diatom frustules has the potential to provide information on Fe-availability in past oceans, and indirectly on $\mathrm{Si}$ burial and $\mathrm{CO}_{2}$ uptake. However, concerns have been raised regarding the possible role of post-mortem diagenesis on the measured frustule $\mathrm{Fe} / \mathrm{Si}$. Here, we study cleaned fossil diatom frustules with the aim to distinguish the original Fe-content, biologically incorporated into the frustule during diatom growth, from secondary Fe originating from terrigenous contamination and diagenesis. By applying nanoSIMS imaging we were able to study the distribution of $\mathrm{Fe}$ and $\mathrm{Al}$ (a marker for diagenesis) in the frustules. We observed that chemical contamination signals were present despite the application of established cleaning techniques. It was further observed that the interior of the frustule had generally lower $\mathrm{Al} / \mathrm{Si}$ values, suggesting reduced diagenetic influences. Based on these results, sample preparation and measuring protocols for Fe/Si determination with SIMS were adjusted by (i) including an additional cleaning step with diluted $\mathrm{HF}$ and (ii) targeting of frustule interiors. These adjustments lowered the $\mathrm{Al} / \mathrm{Si}$ and $\mathrm{Fe} / \mathrm{Si}$ of measured frustules significantly, and should therefore lessen the concerns surrounding diagenetic overprinting of diatom frustules and hence result in a more robust paleoproxy.
\end{abstract}


Reconstructing changes in ocean chemistry from microfossils buried in the sediment is a powerful tool for understanding past climate changes. Over the last few decades, paleoclimate research has focused on studying calcareous microfossils. Unfortunately, carbonates are poorly preserved in large swathes of the ocean floor resulting in significant gaps in the paleoclimate records. To fill these gaps, researchers have searched for alternatives. One such alternative are frustules of diatoms, a ubiquitous group of microalgae that evolved during the Triassic (Sorhannus, 2007). Diatom frustules are constructed from hydrated amorphous silica, which allows them to be preserved in carbonate-poor areas and thus makes them a valuable asset for paleoclimate reconstructions (e.g. Hendry \& Rickaby, 2008).

Diatom frustules buried in the sediment can be used to infer past water-column conditions by studying diatom assemblages (e.g., Crosta et al., 2004), diatom-specific biomarkers such as longchain polyamines (Bridoux \& Ingalls, 2010), or the isotopic and elemental composition of the frustules, such as $\delta^{30} \mathrm{Si}$ (De La Rocha et al., 1997), $\delta^{15} \mathrm{~N}$ (Robinson et al., 2004) and Zn/Si ratios (Hendry \& Rickaby, 2008). Recently, Pichevin et al. (2014) observed a direct correlation between dissolved $\mathrm{Fe}$ concentrations in surface sea water and frustule $\mathrm{Fe} / \mathrm{Si}$, leading to the introduction of frustule $\mathrm{Fe} / \mathrm{Si}$ ratios as a novel proxy to track iron-availability during past diatom growth. The authors reported frustule-bound Fe concentrations in a sediment core from an upwelling region (the Gulf of California), and used these to link dissolved Fe in surface water with sediment biogenic silica burial. Interestingly, the $\mathrm{Fe} / \mathrm{Si}$ values measured in summer month diatom traps were significantly higher than those measured in the sediment, indicating an accumulation bias in the sediment towards winter conditions. Moreover, by coupling the novel Fe/Si measurements to other established diatom-bound geochemical tracers such as silicon isotopes and bulk biogenic silica wt $\%$, Pichevin et al. (2014) obtained information on the role of iron-availability in upwelling regions.

Understanding changes in iron-availability is of interest because Fe is a key nutrient for primary producers and is closely associated with the presence of several macronutrients such as $\mathrm{N}$ and $\mathrm{P}$ as well as $\mathrm{Si}(\mathrm{OH})_{4}$. During winter upwelling events, replete macronutrients can induce Fe limitation and stress, which can in turn lead to more heavily silicified diatoms and eventually greater Si burial (Hutchins \& Bruland, 1998; Takeda, 1998). Accordingly, climatic periods marked by damped winter upwelling conditions may maintain Fe-replete conditions and thus exhibit reduced Si burial. Since changes in diatom frustule silicification and Si-burial have been shown to impact the oceanic carbon cycle via changes in the transport of organic carbon (Arellano-Torres et al., 2011; Pichevin et al., 2012), the study of $\mathrm{Fe} / \mathrm{Si}$ in frustules may give insights into changes in $\mathrm{CO}_{2}$ uptake in past upwelling regions. This may even be expanded to High Nutrient Low Chlorophyll (HNLC) areas, which are important for biogenic silica (opal) burial, accounting for as much as two thirds of the Si sink in modern oceans (DeMaster, 2002). Changes in Si burial in these areas can have large influences on $\mathrm{pCO}_{2}$ levels (Dymond and Lyle, 1985; Sigman and Boyle, 2000). Today, HNLC areas are subject to severe Fe-limitation, but it is thought that this has varied over time (Arellano-Torres et al., 2011). The possibility to reliably monitor Fe-availability in the geological past would greatly enhance our understanding of these different mechanisms.

Even though the exact physiological mechanisms behind Fe incorporation into diatom frustules are presently unknown, it has been observed that Fe is structurally incorporated into the frustule during diatom biosynthesis (Ingall et al., 2013; Yuan et al., 2019). However, frustules may also obtain additional Fe during post-mortem diagenetic processes (Yuan et al., 2019; Ren et al., 2013). An important marker to check for potential diagenesis is the concentration of $\mathrm{Al}$ present in the 
frustule. Research has shown that $\mathrm{Al}$, originating from detrital materials, can become structurally incorporated into the silica framework (van Capellen \& Qiu, 1997; Dixit et al., 2001). Thus, Al/ Si ratios in diatom frustules found in the sediments may exceed that of primary uptake during biosynthesis ( $\mathrm{Al} / \mathrm{Si}$ ratios of $\left.10^{-4}-10^{-3}\right)$, as observed in open-ocean assemblages (van Bennekom et al., 1989; Van Bennekom et al., 1991; Koning et al., 2007; Yuan et al., 2019).

To limit diagenetic biasing of Fe/Si determination in frustules, Pichevin et al. (2014) took several measures to minimize the role of secondary $\mathrm{Fe}$ and $\mathrm{Al}$ contaminates. One, they applied published and well-established cleaning protocols for the removal of clays and other terrigenous components (Morley et al., 2004; Hendry and Rickaby, 2008). Two, they used scanning electron microscopy (SEM) to identify and select samples with little detrital contamination. Three, by using an ion microprobe instead of dissolution techniques, they were better able to differentiate between terrigenous coatings and biologically-fixed metals inside the siliceous matrix. Despite these precautions, high concentrations of $\mathrm{Al}$ were nevertheless observed in some samples. Although a lithogenic source for the observed high $\mathrm{Al} / \mathrm{Si}$ could not be excluded, this was unlikely because of the rigorous cleaning protocols and SEM checks employed. Instead, the Al enrichment was likely due to a post-mortem alteration of the silica structure, either by the formation of an aluminosilicate coating on the surface of the frustule or as a strong alteration of the existing silica framework (Koning et al., 2007). Determining the most likely source of $\mathrm{Al}$-contamination, and exploring strategies for its elimination, or at least minimalization of its influence on diatom $\mathrm{Fe} / \mathrm{Si}$ ratios, is essential for the advancement of this proxy.

This study aims to distinguish the original Fe-content of the frustule, biologically incorporated into the frustule during diatom growth, from secondary Fe originating from clay contamination and diagenesis. The nanoSIMS imaging technique was applied to determine the distribution of $\mathrm{Fe}$ and $\mathrm{Al}$ within diatom frustules previously studied by Pichevin et al. (2014). Our results showed that Al, a marker for diagenetic influences, was still present in the form of 'hotspots' despite the application of established cleaning techniques. These areas were found to strongly colocalize with higher Fe signals, therefore influencing the final $\mathrm{Fe} / \mathrm{Si}$ signal. Our data further suggested that the frustule interiors, specifically those of centric diatoms, generally have lower $\mathrm{Al} / \mathrm{Si}$ values. Based on these results, an additional cleaning step with diluted $\mathrm{HF}$ was performed to remove the observed surfacebound $\mathrm{Al}$ hotspots followed by ion microprobe measurements targeting frustule interiors of centric diatoms. This approach lowered $\mathrm{Al} / \mathrm{Si}$ by one order of magnitude, suggesting a reduced influence of diagenetic overprinting, and resulted in a more robust paleoproxy to study Fe-availability in upwelling regions and its link to silicon and carbon cycles.

\subsection{Materials \& Methods}

\subsubsection{Materials}

The samples in this study were previously studied by Pichevin et al. (2014). They originate from sediment Core MD-022515 retrieved from the Guaymas Basin $\left(27^{\circ} 29.01 \mathrm{~N} ; 12^{\circ} 04.46 \mathrm{~W} ; 881 \mathrm{~m}\right.$ water depth) during the MONA (Marges Ouest Nord Américaines) cruise of the R/V Marion Dufresne (International Marine Global Changes-IMAGES VIII) in June 2002 (Pichevin et al. 2012). The dataset generated by Pichevin et al. (2014) covers the range of 31,347- 6840 years BP and includes records of diatom $\mathrm{Fe} / \mathrm{Si}$, diatom $\delta^{30} \mathrm{Si}$, bulk biogenic silica $(\mathrm{wt} \%$ ) and the $\mathrm{Si} / \mathrm{Corg}$ ratio of the bulk sediment. For this study we selected a subset of ten samples covering a time interval of 20,354-8739 years BP. 
The frustules used for this study were previously cleaned for the Pichevin et al. (2014) study. This was done by first performing mechanical separation (e.g., sieving), after which the diatom frustules were cleaned following established and published protocols (Morley et al., 2004; Hendry and Rickaby 2008) that included both mechanical and chemical cleaning steps (for further details see Pichevin et al., 2012; Pichevin et al., 2014). For the nanoSIMS measurements in this study, no additional cleaning steps were taken.

\subsubsection{Sample preparation and NanoSIMS imaging}

A needle-tip portion of the thoroughly cleaned diatom frustules was pressed on double-sided conductive carbon tape attached to a SEM pin stub. The sample was subsequently coated with a $12 \mathrm{~nm}$ thick Au layer using a sputter coater (JOEL JFG-2300HR high-resolution fine coater, JEOL FC-TM20 thickness controller). Finally, the sample was imaged with a table-top SEM (JEOL JCM-6000PLUS NeoScope Benchtop SEM) operating at a 10-kV accelerating voltage to identify specimens suitable for nanoSIMS analysis (individual frustules with minimal 3D topography).

Nanoscale secondary ion mass spectrometry was performed with the nanoSIMS 50L instrument (Cameca) operated at Utrecht University. Electron multiplier detectors were set using a standard (SPI Supplies, 02757-AB 59 Metals \& Minerals Standard) to enable the detection of secondary ions $24 \mathrm{Mg}+, 27 \mathrm{Al}+, 28 \mathrm{Si}+, 40 \mathrm{Ca}+, 56 \mathrm{Fe}+, 64 \mathrm{Zn}+$ and $138 \mathrm{Ba}+$. Of these ions, $27 \mathrm{Al}+, 28 \mathrm{Si}+$ and $56 \mathrm{Fe}+$ are the main focus of this study, while the other ions were used to aid the identification of possible contamination by particulate clay and other non-opaline phases (Morley et al., 2004; Ellwood and Hunter, 1999; Shemesh et al., 1988). Sputtering was done with the $\mathrm{O}^{-}$primary ion beam generated by the duoplasmatron oxygen source. Prior to the analysis of each target diatom frustule, the measurement area was pre-sputtered with the primary ion beam (FCo current 20pA) until stable secondary ion yields were established. Stable ion yields proved difficult to achieve so a very thorough pre-sputtering protocol was established. During pre-sputtering diaphragm settings were first set to D1-0, with C4y set to 50 bits to avoid detector overloading, and the pre-sputtering was monitored until the counts at detector \#3 (28Si) reached $\sim 170 \mathrm{kcps}$. This continued with the diaphragm switched to D1-1 until stable secondary ion counts were reached. After pre-sputtering, analysis was carried out in the imaging mode by rastering a high-energy $\mathrm{O}^{-}$beam $(16 \mathrm{keV}, 20 \mathrm{pA}$ FCo, nominal beamsize 300-600 nm) over a target area on the frustule (between $4 \times 4 \mu \mathrm{m}$ and $55 \mathrm{x}$ $55 \mu \mathrm{m}$ in size) and measuring the sputtered secondary ions at a resolution of either $128 \times 128$ or 256 x 256 pixels and with a dwell time of $1 \mathrm{~ms}$ /pixel The diaphragm and slit settings were D1-3, ES-3, AS-2, EnS-1. Target areas were measured multiple times (typically over 300 planes) to provide depth resolution and thus assess potential surface contamination. The combination of the primary ion current, dwell time and number of measured planes did not allow measurements through the entire thickness of the frustule.

Since nanoSIMS allowed the analysis of individual frustules, initial measurements targeted different species of centric or pennate diatoms, radiolaria, and silicoflagellates. In the course of these initial tests, however, radiolaria and silicoflagellates proved difficult to measure as their pronounced 3D topography made it difficult to tune the instrument. Pennate frustules were, at times, successfully measured but the collected data was insufficient to draw conclusions. Consequently, our analysis focuses on frustules of centric diatoms, which were rather straightforward to measure. 


\subsubsection{SIMS analysis}

The samples that were previously cleaned for the Pichevin et al. (2014) record, and measured with the nanoSIMS in this study, received an additional washing with diluted $\mathrm{HF}$ to remove the outer layer of silica and with this any remaining surface contamination. Frustules were then cracked by ultrasonication and pressed into an indium mount. Measurements of trace metals took place with the ims-4f Ion Microprobe (Cameca) operated at the NERC (National Environment Research Council) Microprobe Facility, School of Geosciences, University of Edinburgh. Specifically, samples 2926, 1591, 1160 and 1106 were remeasured. The clean diatom samples were pressed into an indium foil and 12-14 frustules were analysed for up to 10 cycles with the ion microprobe. Only frustule interiors were targeted in these measurements. A $10 \mathrm{nA}$ primary beam was used with a 25-micron image field and energy filtering $(75 \mathrm{eV})$. The metals $\left({ }^{23} \mathrm{Na},{ }^{26} \mathrm{Mg},{ }^{27} \mathrm{Al},{ }^{39} \mathrm{~K},{ }^{54} \mathrm{Fe},{ }^{138} \mathrm{Ba}\right)$ were normalised over $\mathrm{SiO}_{2}$, mimicking the earlier measurements performed for the Pichevin et al. (2014) study. The final metal: $\mathrm{SiO}_{2}$ values represent the average of the 12-14 measurements on centric diatom frustules made for each sample.

\subsection{Results}

This study aims to distinguish the original Fe-content, biologically incorporated into the frustule during diatom growth, from secondary Fe originating from (terrigenous) clay contamination and diagenesis. To do this, an explorative approach with nanoSIMS imaging was used to identify the distribution of $\mathrm{Fe}$ and $\mathrm{Al}$. Based on these results, additional measurements were performed with the ion microprobe.

\subsubsection{Distribution of $\mathrm{Fe}$ and $\mathrm{Al}$ in cleaned fossil diatom frustules}

NanoSIMS images of individual diatom frustules revealed the presence of $\mathrm{Al}$ and Fe-rich 'hotspots' (Fig. 2.1). We interpret these areas as contamination. Some of the hotspots could not be associated with anything in the corresponding SEM images. Some of the smaller areas with highintensity a signals were situated around the pores and could be distinguished in the SEM images as a narrowing of the pore (Fig. 2.1a), suggesting a deposition of aluminosilicates. Another larger presence of $\mathrm{Al}$ on the frustule appeared to be due to 'loose' terrigenous debris stuck to the frustule. This presence was also most closely associated with high Fe signals (Fig. 2.1b).

\subsubsection{Analysis of diatom frustule interiors vs. exteriors}

To minimize the influence of the contamination on the Fe/Si ratios in the frustules, we exploited the high lateral and depth resolution of the nanoSIMS measurements and processed the image stacks in three steps (see Supplement 1). First, regions of interests (ROIs) corresponding to the $\mathrm{Al}$ and Fe-rich hotspots were removed from the analysis. This generally led to a significantly lower $\mathrm{Fe} / \mathrm{Si}$ ratio in the frustule. Second, variation of the Fe/Si ratios with depth was checked in each individual frustule. Such variation could occur due to insufficient removal of surface contamination by pre-sputtering, or due to insufficient stability of the secondary ion yields caused by insufficient implantation of the primary O- ions into the frustule matrix. If the coefficient of variance of this depth profile exceeded $20 \%$, the frustule was excluded from further analysis. The last step was based on a purely statistical treatment, and involved removal of outliers based on the $\mathrm{Al} / \mathrm{Si}$ ratio. This was performed only on $\mathrm{Al} / \mathrm{Si}$ as this ratio is seen as a marker for strong diagenetic influences. 
a)

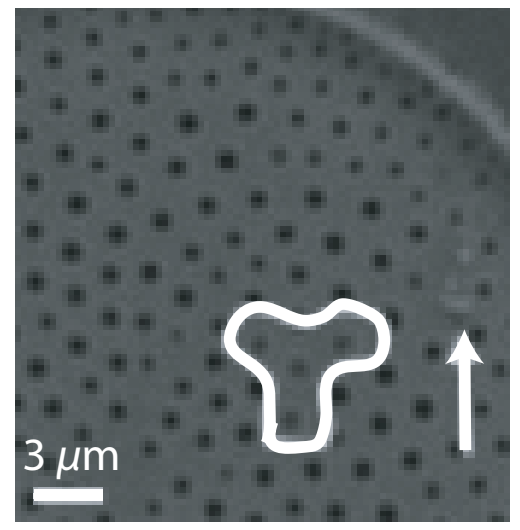

c)

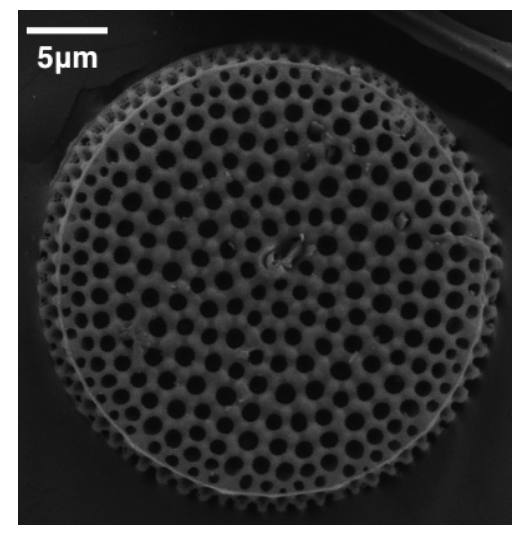

b)

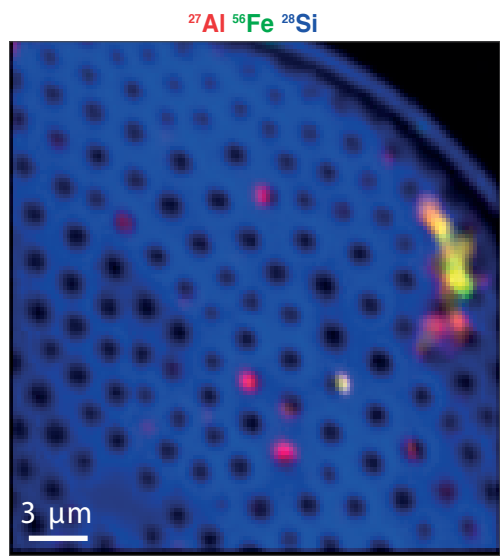

RGB frustule interior

d)

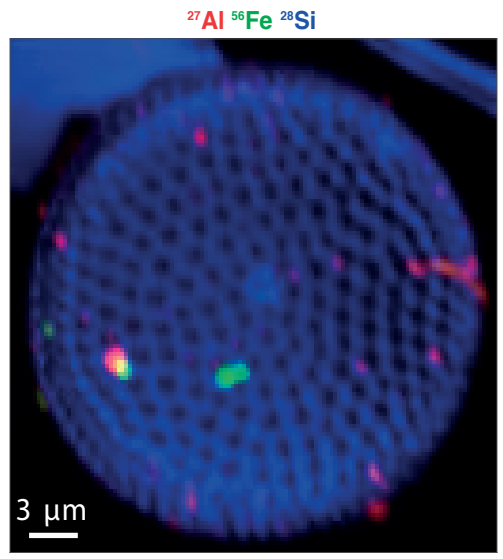

RGB frustule exterior

Figure 2.1: Imaging of individual frustules of fossil diatoms. Shown are SEM images (left) and the corresponding nanoSIMS images (right) of a frustule interior (top) and exterior (bottom). Outline in image (a) marks narrowing of pores, arrow points to loose particles. NanoSIMS images of the Al, Fe and Si ion counts were overlaid in a single RGB image. Individual ion count images are shown in Supplement 1. 
Out of the total of 140 frustules measured, this procedure resulted in quality-checked $\mathrm{Al} / \mathrm{Si}$ and $\mathrm{Fe} / \mathrm{Si}$ ratios for 75 individual frustules (Fig. 2.2).

This data was analysed by ANOVA with exterior/interior considered as a fixed factor and the sample set as the random factor (Supplement 2). The analysis revealed that there was a significant main effect of the exterior/interior factor for $\mathrm{Al} / \mathrm{Si}(\mathrm{p}=0.036$; Table $\mathrm{S} 2.3)$, with frustule exteriors showing significantly higher $\mathrm{Al} / \mathrm{Si}$ ratios than the frustule interiors. The interaction term was not significant $(\mathrm{p}=0.128$; Table $\mathrm{S} 2.3)$, indicating that this difference was consistent among all samples. For the $\mathrm{Fe} / \mathrm{Si}$ ratios the main effect of the exterior/interior factor was also significant $(\mathrm{p}=0.03$; Table S2.4). In this case, however, the interaction term was significant ( $p=0.003$; Table S2.4). A post-hoc analysis (Table S2.5) revealed that that frustule exteriors had significantly higher Fe/ $\mathrm{Si}$ ratios than frustule interiors in 3 out of 11 samples (2926, 1466, 1106), while no significant differences between exteriors and interiors were found in the remaining 8 samples (primarily due to insufficient number of replicate measurements). The interpretation of the difference between frustule interior and exterior for the $\mathrm{Fe} / \mathrm{Si}$ ratio needs to be done with caution as the homoscedasticity condition was not met. Comparison of the centric diatoms based on their size revealed no significant differences (data not shown).

\subsubsection{SIMS measurements with the novel approach}

The presence of Al-rich hotspots observed by nanoSIMS revealed the remaining influence of diagenetic overprints and contamination despite the application of well-established cleaning protocols. Therefore, frustules from selected samples were additionally cleaned with diluted HF and remeasured by conventional SIMS. To account for the apparent differences between frustule interiors and exteriors, these additional measurements exclusively targeted frustule interiors.

The novel measurement approach significantly reduced $\mathrm{Al} / \mathrm{Si}$ values by 82 to $90 \%$, suggesting a strongly reduced diagenetic presence (Table 2.1). A similar decrease for $\mathrm{Fe} / \mathrm{Si}$ ratios (84 to 88\%) was also observed. These results show that the impact of the cleaning was very substantial. Despite this, the downcore trend observed in the originally published Fe/Si record remains comparable (see Fig. 2.3)

Table 2.1: Al and Fe content in fossil diatom frustules obtained by conventional SIMS. Shown are calibrated molar ratios measured in 10-14 individual frustules by the original approach of Pichevin et al. (2014) and by our novel approach involving an additional HF cleaning step and targeting of frustule interiors.

\begin{tabular}{lccccc}
\hline Core depth $(\mathrm{cm})$ & Approach & $\begin{array}{c}\mathrm{Al}: \mathrm{SiO}_{2} \text { ppm } \\
(\mathbf{n}=10-14)\end{array}$ & $\pm \mathrm{SD}$ & $\begin{array}{c}\mathrm{Fe}: \mathrm{SiO}_{2} \mathbf{p p m} \\
(\mathbf{n}=10-14)\end{array}$ & \pm SD \\
\hline $\mathbf{1 1 0 6}$ & Original & 1561 & 454 & 441 & 182 \\
& Novel & 288 & 291 & 51 & 44 \\
1161 & Original & 4215 & 2430 & 563 & 191 \\
& Novel & 609 & 450 & 88 & 53 \\
1491 & Original & 437 & 155 & 82 & 19 \\
& Novel & 44 & 32 & 13 & 6 \\
2926 & Original & 964 & 853 & 206 & 68 \\
& Novel & 151 & 159 & 31 & 19 \\
\hline
\end{tabular}


a)

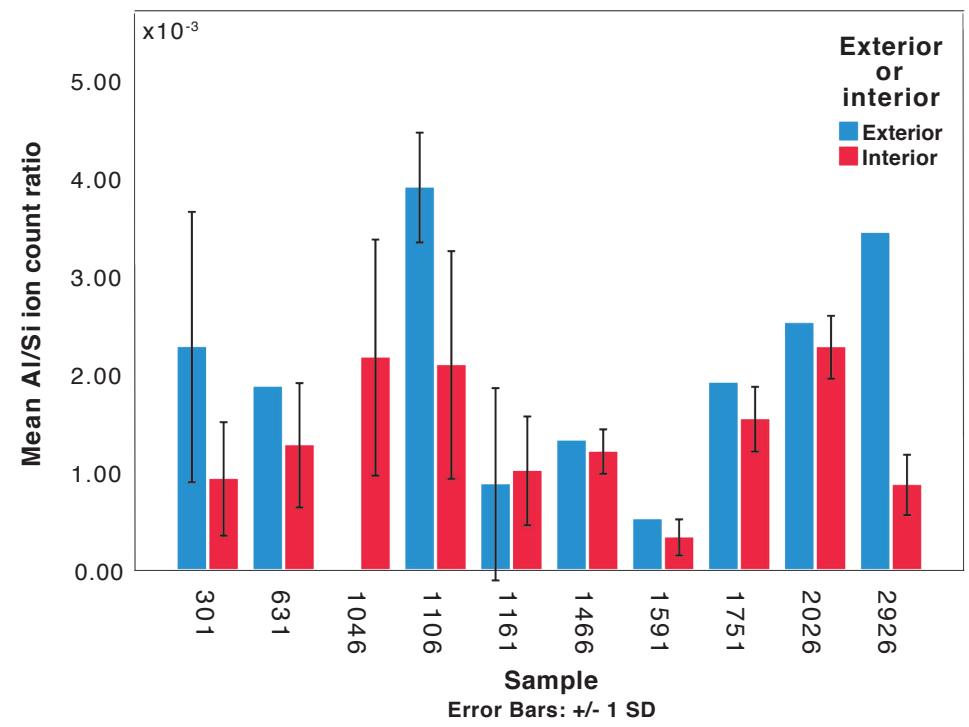

b)

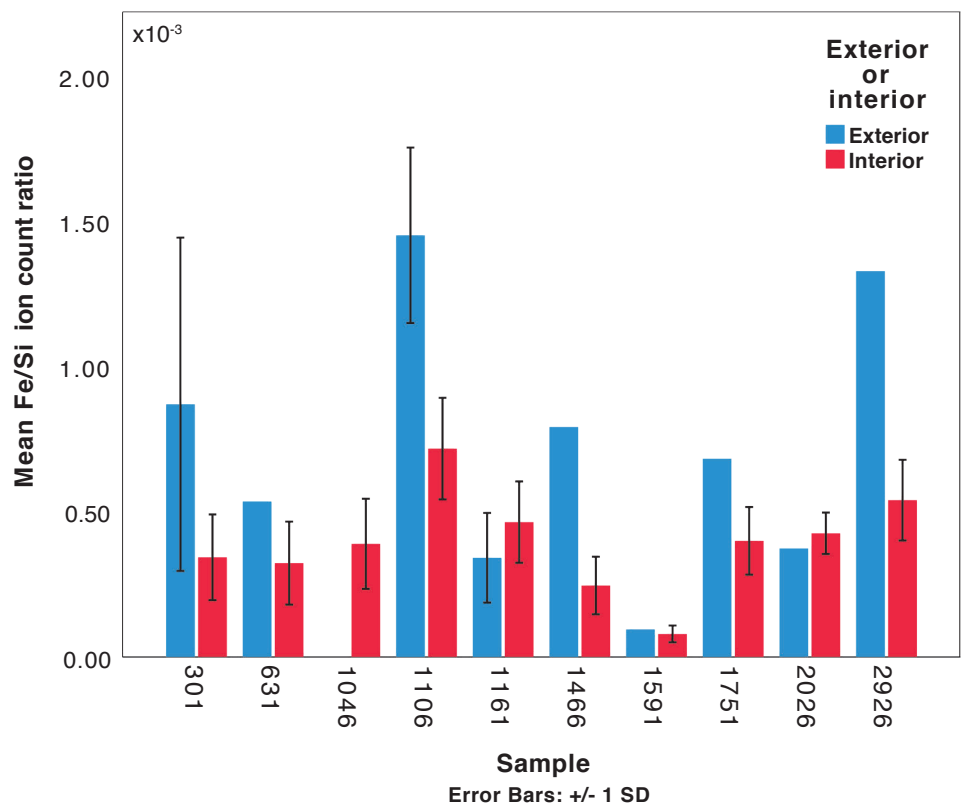

Figure 2.2: Comparison between frustule interior and exterior metal/Si ratios Data represents values after data processing steps as discussed in Supplement 1; a) Al/Si means and SD throughout the record; b) Fe/Si means and SD throughout the record 


\subsection{Discussion}

The $\mathrm{Fe} / \mathrm{Si}$ ratio of diatom frustules has great paleoproxy potential, but concerns have been raised regarding the role of sedimentary post-mortem diagenesis in influencing the measured values. In this paper we use high-resolution correlative imaging to evaluate these concerns. Based on our results we propose several steps that can be undertaken to reduce the uncertainty of the proxy.

\subsubsection{Aluminium contamination in fossil diatom frustules}

Research has shown a discrepancy between frustule $\mathrm{Al} / \mathrm{Si}$ ratios in specimens retrieved from surface waters and those found in the sediment (Van Bennekom et al., 1989). This discrepancy has been attributed to aluminium originating from detrital material, which can alter the silica framework (van Capellen \& Qiu, 1997; Dixit et al., 2001). To reduce the impact of this diagenetic overprint on metal/Si ratios, the samples in this study have been thoroughly cleaned with established protocols (see Morley et al., 2004; Pichevin et al., 2014) and have been checked with SEM analysis for visible clay contamination. In addition to this, trap data from Pichevin et al. (2014) showed frustule Fe/Si values similar or greater than those measured in the sediment, suggesting that the diagenetic effect in the sediment was not very strong. Despite these extensive precautions, detailed spatially resolved elemental data produced with the nanoSIMS revealed an undeniable presence of Al-rich areas on the frustule surface which colocalized with increased Fe-signals. We interpret these enrichments as residual contamination. The source of many of the Al-rich hotspots could not be identified with SEM analysis, while others could be associated with a narrowing of the pores or as loose particles on the surface. The degree to which the removal of these Al-hotspots during data-processing led to a decrease in the frustule $\mathrm{Al} / \mathrm{Si}$ differed greatly between frustules (between $\sim 4$ and $\sim 70 \%$; Fig. $\mathrm{S} 2.4)$.

The irregular presence, placement and magnitude of the Al-hotspots, and their influence on the frustule $\mathrm{Al} / \mathrm{Si}$ ratios, suggest that the $\mathrm{Al}$ contamination we observe is predominantly present in the form of aluminosilicate deposits on the frustule surface instead of the structural incorporation of $\mathrm{Al}$ into the silica matrix. This is further supported by the observed differences between the frustule interior and exterior, as a structural incorporation would most likely lead to a more homogenous distribution. The suggestion that surface aluminosilicate precipitates are the main diagenetic product is in agreement with recent research (Koning et al., 2007; Ren et al., 2013). Evidence suggesting structural incorporation of $\mathrm{Al}$ has been presented in the literature (e.g. Dixit et al., 2001) and cannot be ignored, but it is unlikely that this increases the Fe/ $\mathrm{Si}$ ratios of frustules. It should be considered that $\mathrm{Al}$ can be incorporated into the frustule during diagenesis because its properties are similar to $\mathrm{Si}$; as this is not the case for $\mathrm{Fe}$, it is unlikely that this incorporation mechanism can be linked to the secondary Fe presence. Instead, aluminosilicate precipitates and terrigenous contamination seem to have a dominant role in increasing the metal/Si ratios of the fossil diatom frustules above the natural abundance. Considering the observed presence of surface $\mathrm{Al}$ contamination in hard-to-reach areas such as the pores, a more severe cleaning protocol would be beneficial and was therefore applied to later SIMS measurements.

\subsubsection{Protective nature of the diatom frustule}

By using the nanoSIMS imaging approach, it is possible to identify and remove possible contamination signals and to determine the chemical composition of the residual silica matrix. The underlying silica shows a statistically significant trend towards lower $\mathrm{Al} / \mathrm{Si}$ values when studying the interior of the diatom frustule, compared to the exterior of the frustule, suggesting a reduced 
diagenetic impact for the interior. These observations greatly improve the applicability of the $\mathrm{Fe} / \mathrm{Si}$ proxy as this suggests that lower-resolution techniques may be applied for better count yields, and with fewer contamination concerns, as long as the frustule interior is targeted.

The observed differences between the frustule interior and exterior can likely be linked to the post-mortem diagenesis susceptibility and contamination. As long as the diatom frustule remains intact in the sediment, the inside of the centric diatom appears to remain better protected. However, if the frustule breaks when still in the sediment, the frustule interior may also become susceptible to diagenesis. Therefore, in order to avoid strongly altered specimens, $\mathrm{Al} / \mathrm{Si}$ boundary values should be maintained. Even with this precaution, every imaged diatom frustule contained at least some Al-hotspots. Thus, even when the frustule remains intact, it cannot be guaranteed that the inside of the diatom is unaffected by diagenesis. Diagenetic effects may be caused by an exchange through the pores with surrounding pore waters. This mechanism is supported by the observed presence of aluminum contaminations (interpreted as aluminosilicates) around the pores. The results from this study suggest that the influence of this on the interior of the frustule is limited, but the exact degree to which this may occur is something that needs to be studied further. Until this is fully understood, an extra cleaning step with diluted HF is recommended in order to remove any superficial contamination still present on the surface of the silica.

Without additional data, potential alternative drivers of the signal such as a species effect, frustule size or growing-stage bias cannot be ruled out. With the nanoSIMS it is possible to target individual diatom frustules, making it certain that only centric diatoms are targeted. With the SIMS method, it may happen that when targeting clusters, other species such as pennate diatoms and radiolarians are part of the measurement and may, therefore, influence the data. Even though the nanoSIMS data did not show a conclusive bias, care should be taken to ensure that only a single type of diatom is targeted. Moreover, despite not observing a bias, similarly sized diatom frustules should be targeted to rule out any uncertainty due to species or ontogeny.

\subsubsection{SIMS measurements: applying the new approach}

We propose that an additional HF cleaning step and the targeting of frustule interiors is sufficient to remove contamination concerns for the Fe/Si proxy. In order to test the suggested additions, conventional SIMS measurements were performed, using this approach, on frustules from samples that were previously measured for both the published SIMS record (Pichevin et al., 2014) as well as the nanoSIMS record from this study (Fig. S2.12). These measurements showed that the revised approach yielded significantly lower $\mathrm{Al} / \mathrm{Si}$ values, showing a strongly reduced diagenetic presence. On average, the $\mathrm{Al} / \mathrm{Si}$ ratios decreased by $85 \%$ resulting in ratios considerably lower than the generally accepted contamination levels. With the $\mathrm{Al} / \mathrm{Si}$ ratios, the $\mathrm{Fe} / \mathrm{Si}$ ratios were also lowered considerably (see Table 2.1). Interestingly, while the metal/Si ratios are significantly lower in the SIMS measurements after the additional HF treatment, the relative changes between samples are comparable to those in the Pichevin et al. (2014) record (Fig. 2.3). 


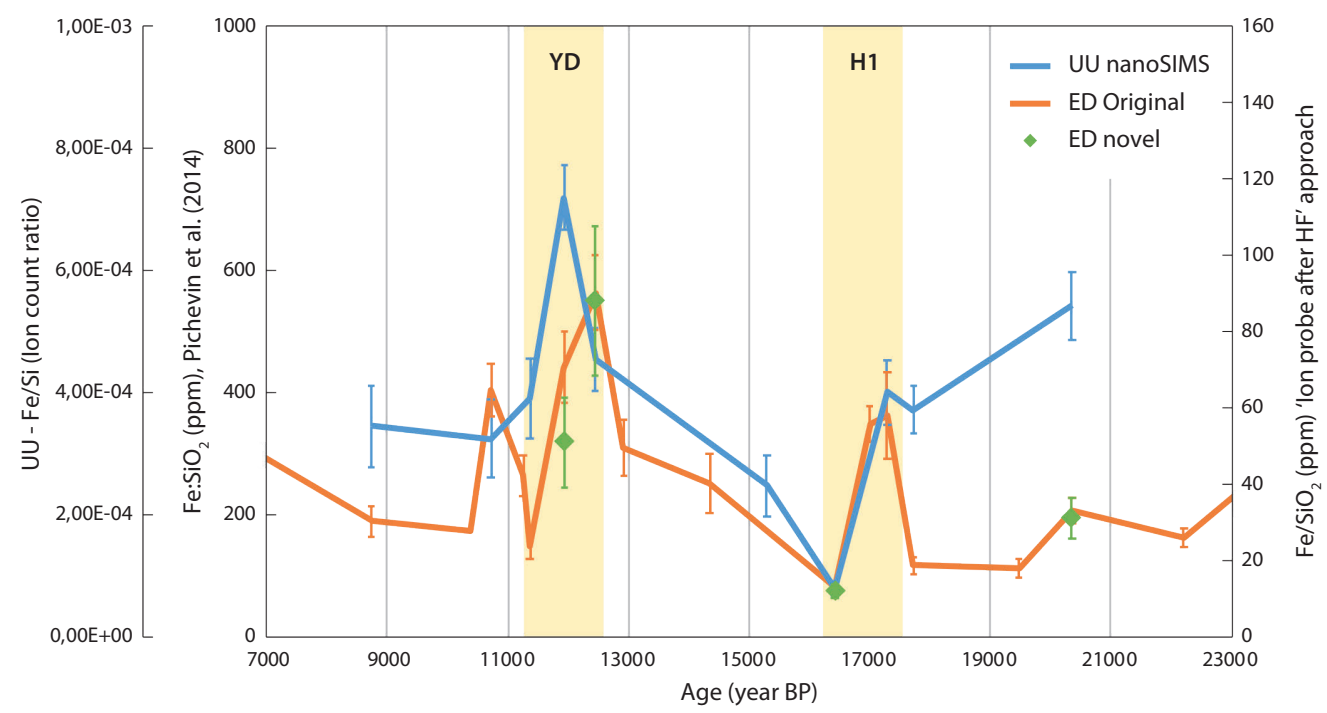

Figure 2.3: Comparison between Fe/Si records UU nanoSIMS record (ion count ratio), original ED (Pichevin et al., 2014) and new ED after HF approach (Calibrated molar ratios). Error bars represent SE. The Heinrich 1 (H1) and Younger Dryas (YD) cold events are marked

\subsubsection{Implications and applications}

The results from both the nanoSIMS and the revised SIMS method confirmed, for a large part, the trends observed in the Pichevin et al. (2014) Fe/Si record (Fig. 2.3). The novel method did however lower the metal:Si values considerably below accepted contamination levels, suggesting a minimal diagenetic influence. Thus, the addition of an extra cleaning step, together with the targeting of frustule interiors, greatly reduced the uncertainty concerning the potential impact of diagenesis in controlling $\mathrm{Fe} / \mathrm{Si}$ in fossil diatom frustules. As the original down-core trend perseveres in the ultraclean samples, the original Fe/Si proxy approach (Pichevin et al., 2014) in fossil diatom frustules can be applied with more confidence. However, considering that nanoSIMS analysis showed a presence of Al-hotspots on frustules treated with the original approach, and as only four samples were remeasured with the new approach, the methodology described in this manuscript should be applied, when possible, to avoid uncertainty.

The importance of the Fe/Si proxy is that we can identify two main modes: Fe-replete (elevated $\mathrm{Fe} / \mathrm{Si}$ ) and Fe-deplete conditions (base-level $\mathrm{Fe} / \mathrm{Si}$ ). These two modes are likely to be driven primarily by nutrient availability. Replete macronutrient conditions during upwelling events can lead to increased productivity, which in turn can create Fe-stress conditions if additional influx of iron (e.g. from terrestrial sources) does not compensate for the deficiency created by increased Fe-demand (Hutchins \& Bruland, 1998; Pichevin et al., 2014). Instead, diatoms become more heavily silicified under Fe-stress conditions (Hutchins \& Bruland, 1998; Takeda, 1998) and thus $\mathrm{Si}(\mathrm{OH})_{4}$ uptake relative to other macronutrients is high, resulting in low $\mathrm{Fe} / \mathrm{Si}$ and high $\mathrm{Si}: \mathrm{C}_{\text {org. }}$. Being able to identify Fe-replete and Fe-deplete conditions in paleoceans without major diagenesis concerns is therefore a useful addition to the proxy toolbox. 


\subsection{Conclusion}

The application of the $\mathrm{Fe} / \mathrm{Si}$ proxy in fossil diatom frustules has great potential for improving our understanding of changing ocean dynamics in the past. However, some concerns have been raised regarding the role of diagenesis in the sediments on measured frustule $\mathrm{Fe} / \mathrm{Si}$. The nanoSIMS technique has, through high-resolution imaging and spot measurements, shown that possible chemical contamination signals were indeed present despite the application of established cleaning techniques. This contamination appeared to be predominantly present in the form of aluminosilicate precipitates on the surface of the frustule exterior and occasionally terrigenous material. Both of these have the potential to influence measured Fe/Si signals. Therefore, an additional precautionary cleaning step with diluted HF was applied and this is shown to further reduce the presence of diagenetic Al. Together, these steps should greatly reduce any bias originating from diagenetic overprints and improve confidence in the proxy to faithfully reflect changing Fe-availability conditions of surface waters. 


\section{Supplements}

\section{Supplement 2.1 Data quality steps}

To ensure the quality of the nanoSIMS data, several data-quality steps were undertaken to reduce uncertainties from both the sample as well as the analytical techniques. The quality criteria are summarized in Figure S2.1, and the contribution of each processing step is discussed in the following sections.

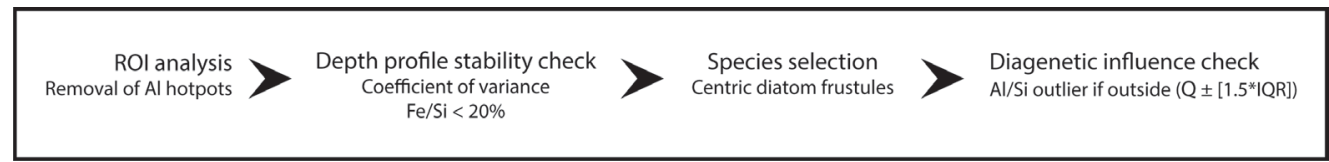

Figure S2.1: Overview of the quality criteria process

ROI removal of metal (predominantly 27Al) hotspots was applied in order to remove possible localized contamination. Next the stability of the measurements was tested. If the coefficient of variation $(\mathrm{COV})$ with depth of the $\mathrm{Fe} / \mathrm{Si}$ ion count ratios exceeded $20 \%$, suggesting an unstable sputtering profile, the data were removed. A species selection was also applied to only include centric diatom frustules. This species selection limits any variability due to species specific Fe uptake or susceptibility towards diagenesis. Finally, outliers in the $\mathrm{Al} / \mathrm{Si}$ data were removed in order to avoid strongly diagenetically altered frustules. This was determined by first carrying out a squareroot transformation to normalize the data and then identifying outliers with SPSS software. The measurements for this manuscript took place during several measuring cycles over a time period of just over a year (late 2017 to early 2019). Over this period a total of 140 measurements were performed. However, several data-quality checks led to a final dataset of 59 measurements on centric interiors.

\section{S.2.1.1 - Removal of contamination hotspots}

Processing of the nanoSIMS data was done using an updated version of the Look@nanoSIMS software (Polerecky et al., 2012). Data-analysis was performed in a structural manner through ROI (region of interest) analysis. By outlining the possible contamination (high-intensity signals from $27 \mathrm{Al}, 56 \mathrm{Fe}, 7 \mathrm{Li}, 24 \mathrm{Mg}, 40 \mathrm{Ca}, 64 \mathrm{Zn}, 136 \mathrm{Ba}$ ) with the ROI tool, these signals could be removed from the frustule's signal. Furthermore, for images involving the frustule interior, possible topography influence of the upright edge of the frustule was avoided by not including this is in the final ROI.

An example showing the different imaged (contamination) ions are presented in Figures S2.2 and S2.3. These are all the measured elements of the frustules shown in section 3.1. There is no consistent co-localization of the different elements, showing that various forms of contamination can exist on the frustule surface. 


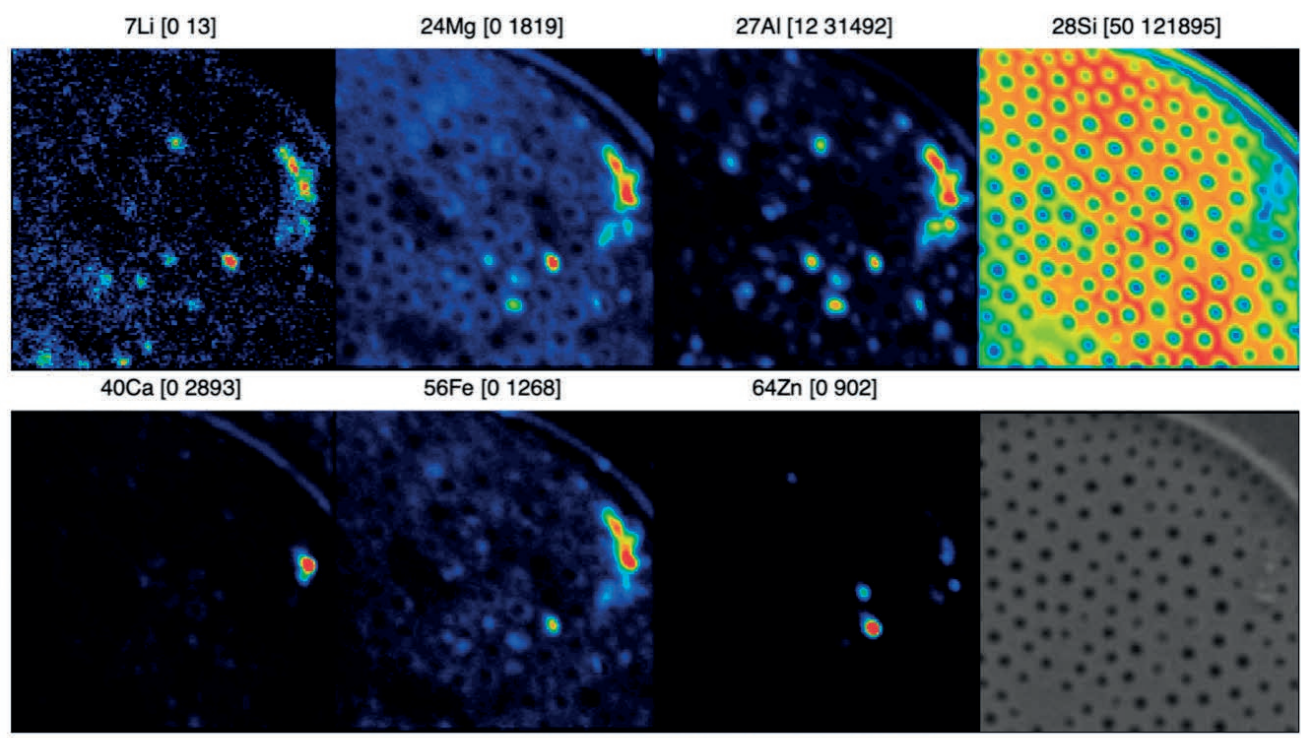

25x25um 128x128pix 300 planes (15.01.19@14:51; $1 \mathrm{~ms}$ )

Figure S2.2: Ion images and corresponding SEM image for a frustule interior Corresponds with Fig. 1 in section 3.1; Red hotspots in the metal ion counts are considered contamination.

$24 \mathrm{Mg}[0$ 4921]

27Al [78 14784]

28Si [68 72852]

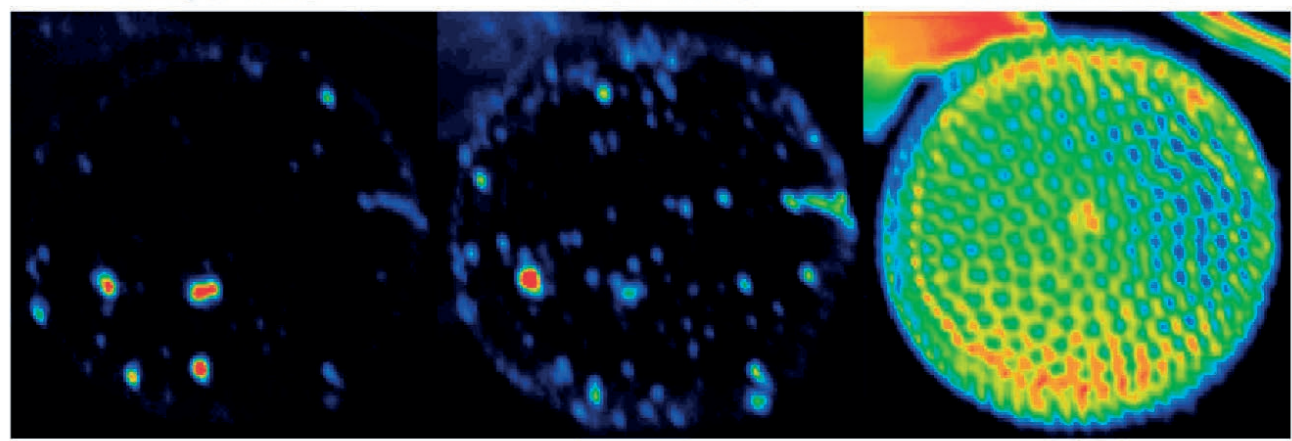

$56 \mathrm{Fe}[0$ 2263]

$64 \mathrm{Zn}[014]$

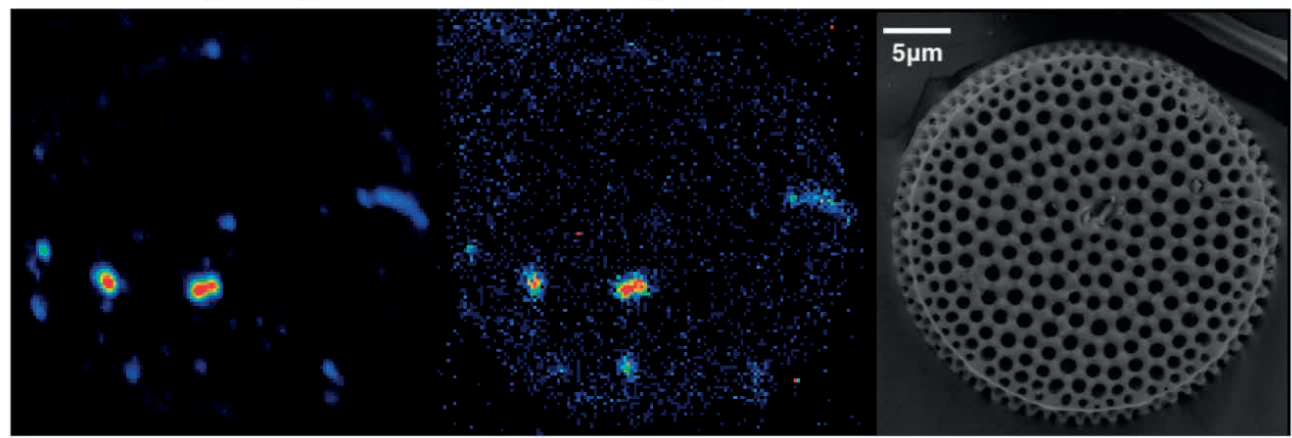

30x30um 128x128pix 300 planes (07.03.18@16:33; $1 \mathrm{~ms})$

Figure S2.3: Ion images and corresponding SEM image for a frustule exterior Corresponds with Fig. 1 in section 3.1; Red hotspots in the metal ion counts are considered contamination. 
a)

All data - influence ROI analysis

- $\mathrm{Fe} / \mathrm{Si}$ after * $\mathrm{Fe} / \mathrm{Si}$ before

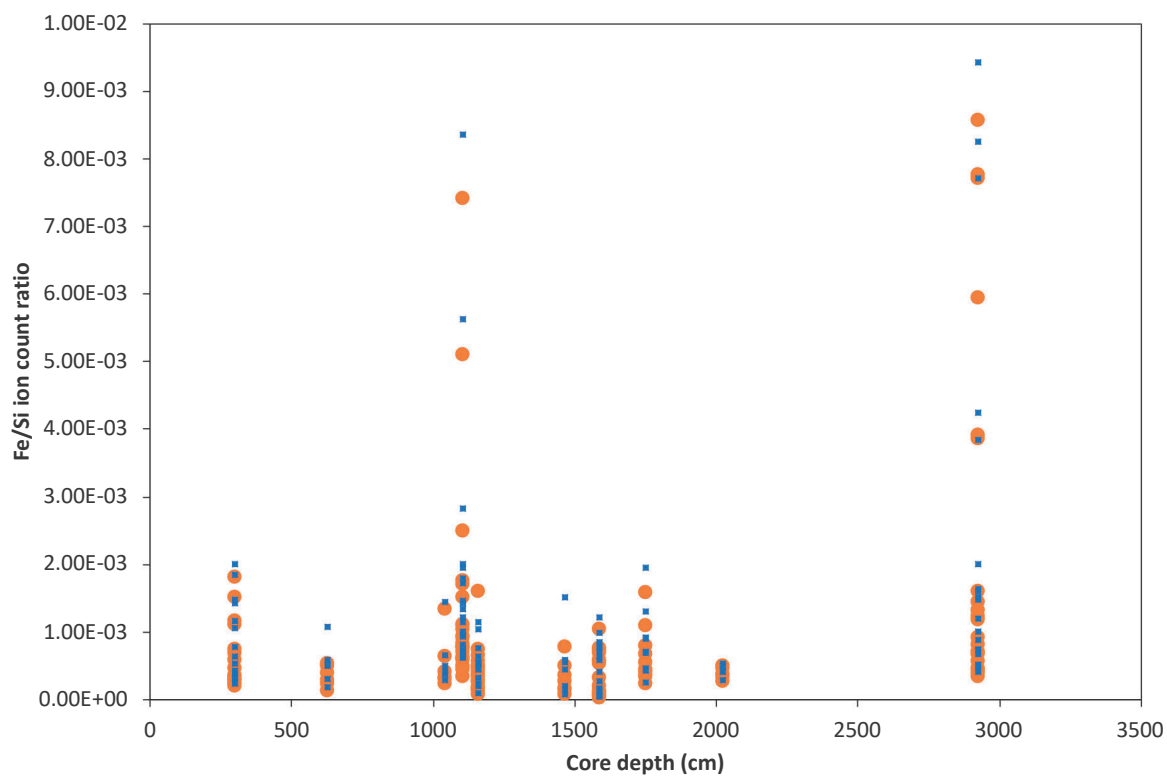

b)

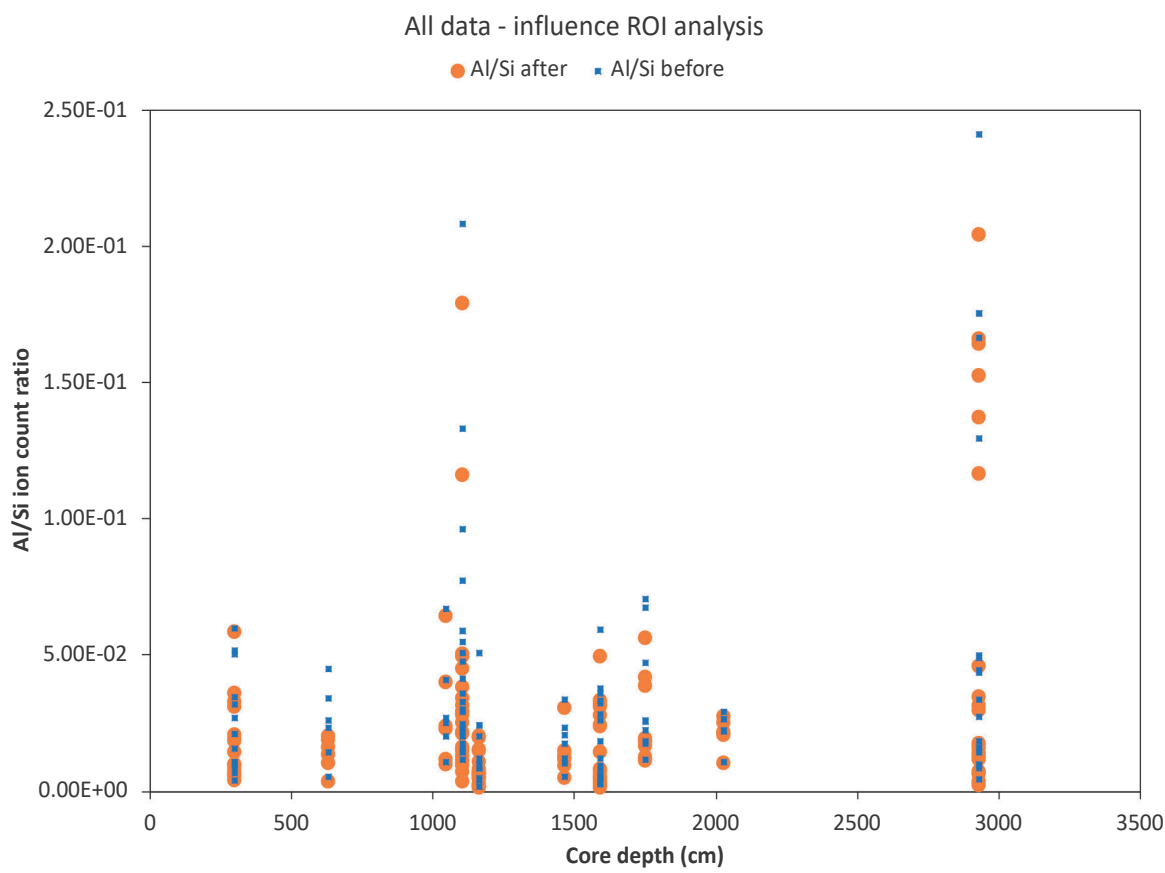

Figure S2.4: Datapoints before and after the removal of contamination ROIs; $\mathbf{N}=140$; This data illustrates the impact of ROI removal on the metal/Si ratios for each measured frustule. This step led to lower metal/Si ratios but the degree at which this occurred varied per frustule. (a) $56 \mathrm{Fe} / 28 \mathrm{Si}$ ion count ratio; (b) $27 \mathrm{Al} / 28 \mathrm{Si}$ ion count ratio 


\section{S.2.1.2. Depth profile stability check}

This check is necessary to evaluate the stability of the measurement. Relatively stable depth profiles are indicative of sufficient pre-sputtering. An example of an unstable sputtering profile (that was removed due to this quality control) is presented in Fig S2.5.
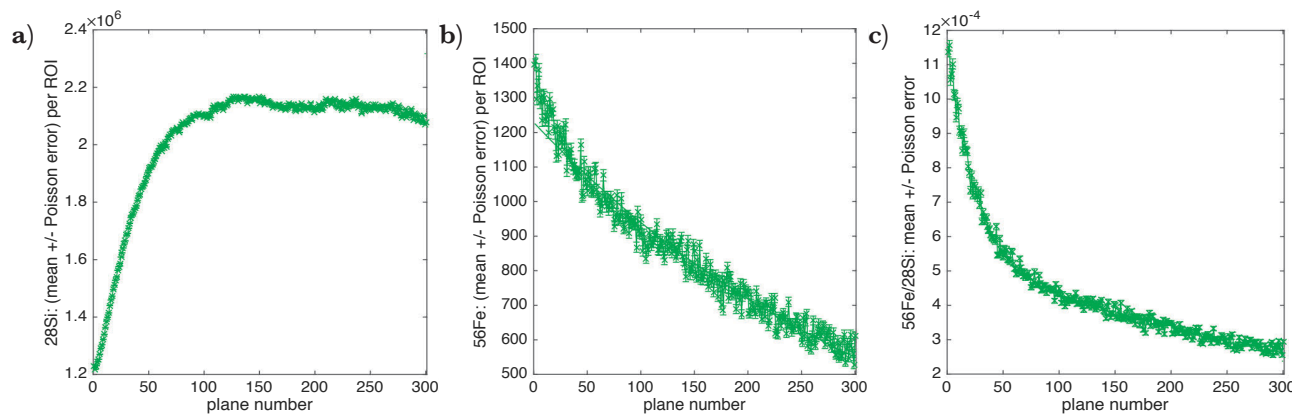

Figure S2.5: Unstable depth profiles in a diatom ROI. Shown are the depth profiles for (a) 28Si ion counts; (b) $56 \mathrm{Fe}$ ion counts; (c) $56 \mathrm{Fe} / 28 \mathrm{Si}$ ion count ratio. The $\mathrm{CV}$ for this ratio profile is $31 \%$. This example shows how an unstable sputtering profile can influence the final ratio of the composite image. The Fe/Si ratio starts at $\sim 12 \mathrm{E}-4$ and reaches $\sim 3 \mathrm{E} 04$ after 300 planes. Even though the rate of change decreases as the silica signal stabilizes after $\sim 100$, the Fe signal does not stabilize within the 300 planes complicating the interpretation of the ratio profile. The removal of unstable sputtering profiles should result in a more reliable dataset. 
a)

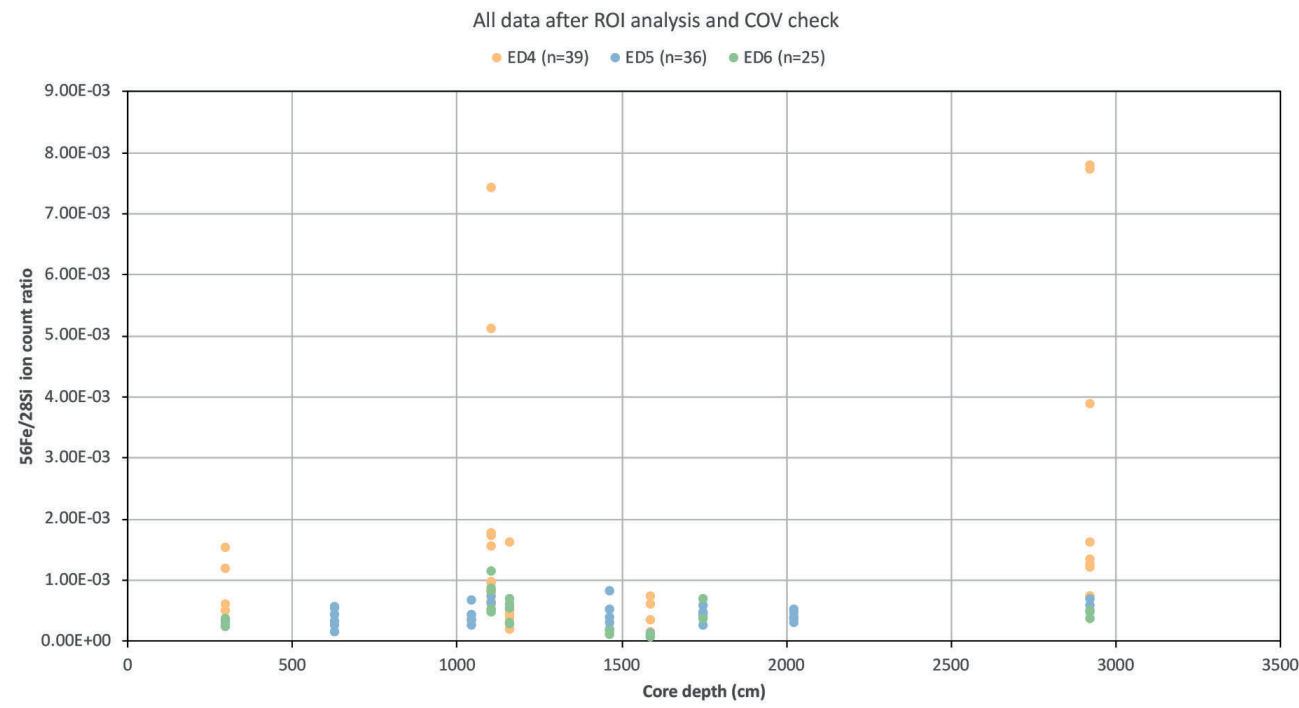

b)

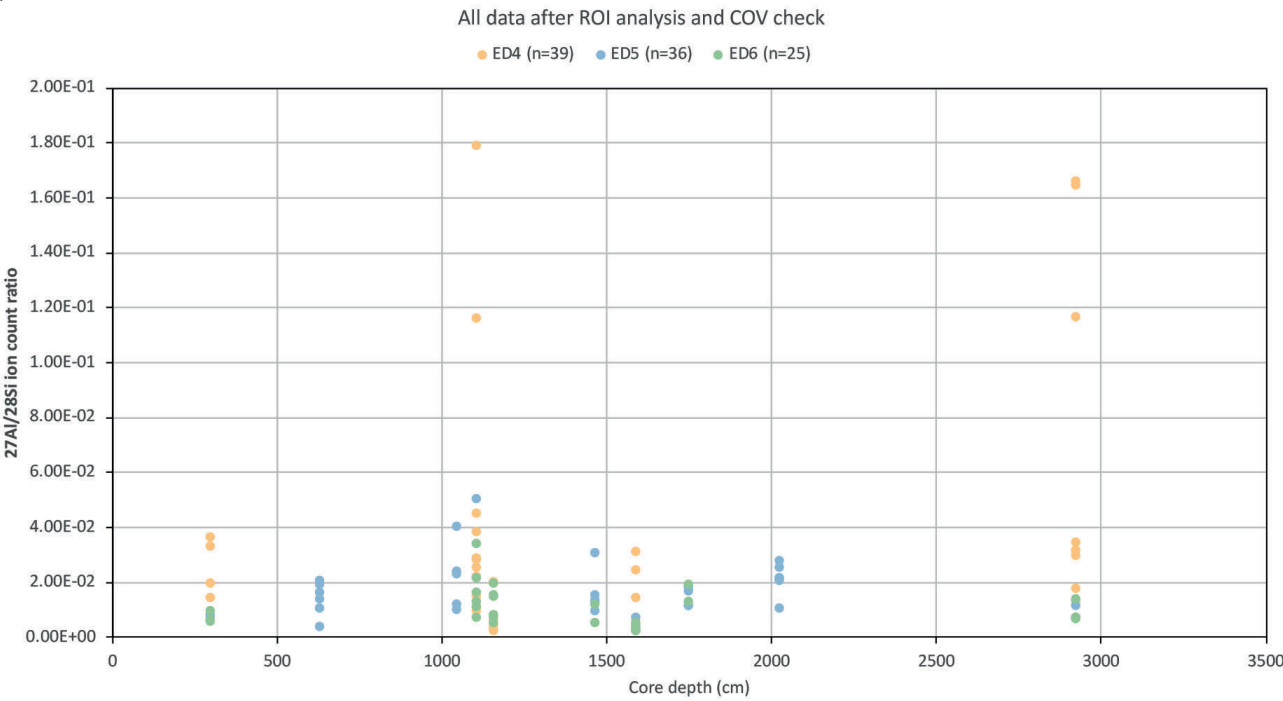

Figure S2.6: Datapoints from three different measuring cycles after removal of contamination

hotspots and the depth profile stability check; $\mathbf{N}=\mathbf{1 0 0}$ In the dataset, the different measuring cycles are shown: Late 2017 (ED4), Early 2018 (ED5) and Early 2019 (ED6). The removal of unstable depth profiles (set at COV Fe/Si $>20 \%$ led to these data-sets (a) Fe/Si ion count ratio; (b) $\mathrm{Al} / \mathrm{Si}$ ion count ratio 


\section{S.2.1.3 Species selection}

Measurements of species other than centric diatoms were removed. These included pennate diatoms, radiolaria and silicoflagellates. Due to this step, 18 measurements were removed.

a)

All data after ROI analysis, COV check and species selection

- ED4 (n=22) - ED5 (n=35) - ED6 (n=25)

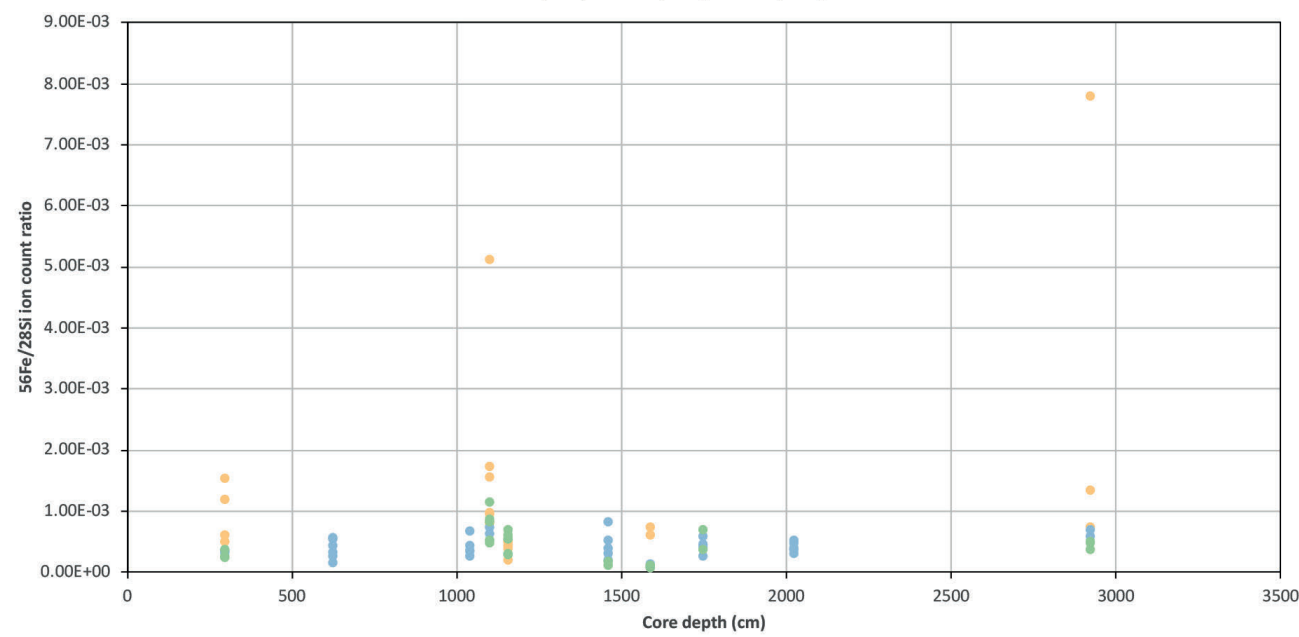

b)

All data after ROI analysis, COV check and species selection - ED4 (n=22) - ED5 ( $n=35)-E D 6(n=25)$

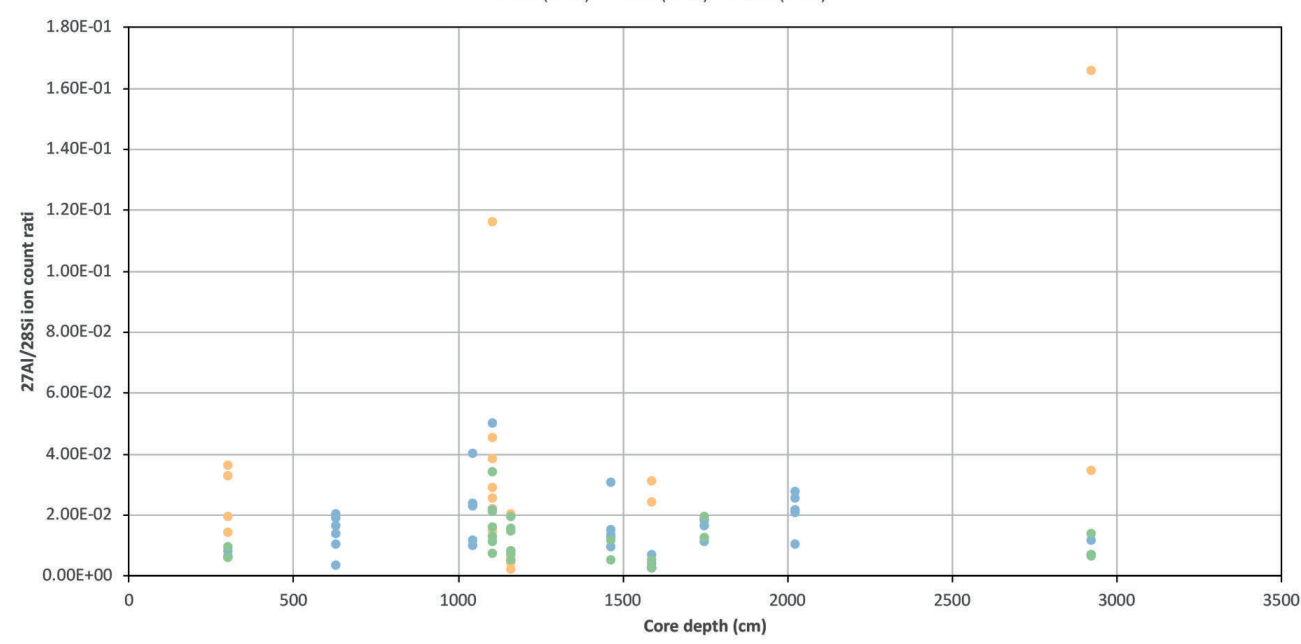

Figure S2.7: Datapoints from three different measuring cycles (ED4; ED5; ED6) after the removal of contamination hotspots, depth profile stability check and species correction; $\mathbf{N}=82$ Shown are the datapoints that represent centric diatom frustules (a) $\mathrm{Fe} / \mathrm{Si}$ ion count ratio (b) $\mathrm{Al} / \mathrm{Si}$ ion count ratio 


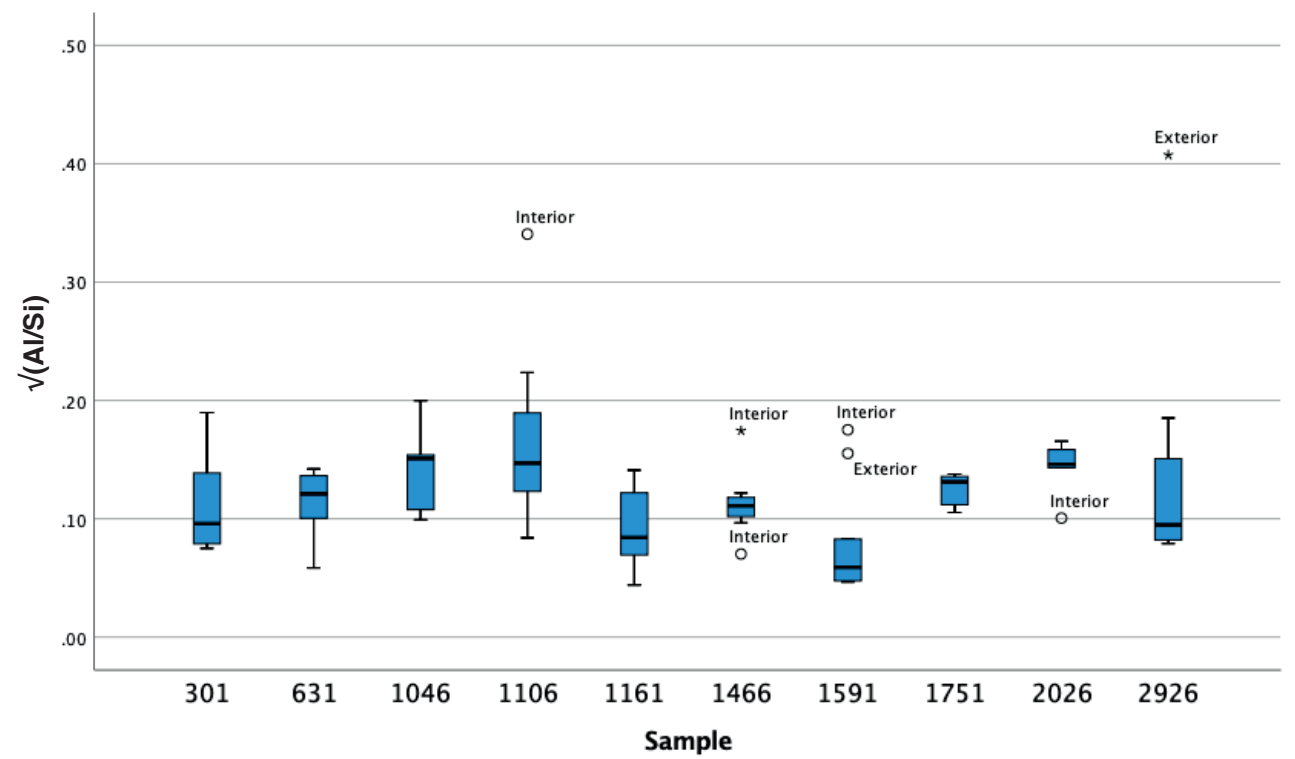

Figure S2.8: Identifying outliers in the square-root transformed A1/Si data. In order to identify strongly altered frustules, the outliers in the data were identified. To account for the varying intrinsic $\mathrm{Al} / \mathrm{Si}$ ion count ratios of the different samples, a square-root transformation was applied to the data.

Outlier $\left({ }^{\circ}\right)=$ Upper/Lower Quartile \pm (Interquartile range*1.5); Extreme outlier $\left(^{*}\right)=$ Upper/Lower Quartile \pm (IQR*3)

a)

All data after ROI analysis, COV check, species selection and outlier removal - ED4 $(n=18)$ - ED5 $(n=33)$ ED6 $(n=24)$

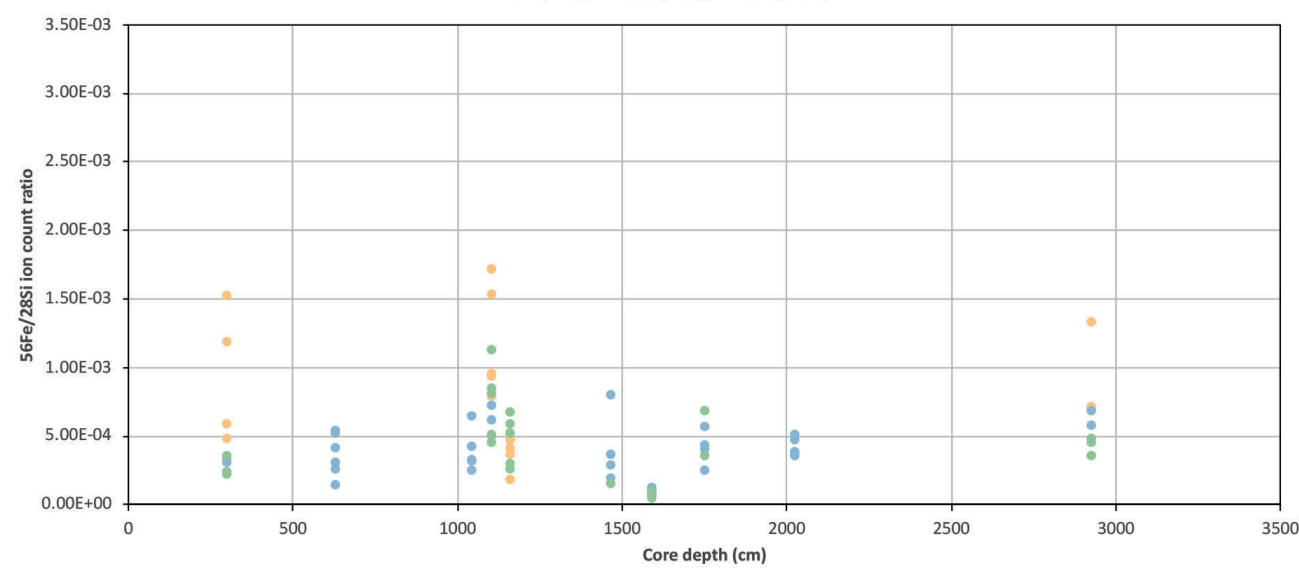


b)

- ED4 ( $n=18) \cdot \operatorname{ED} 5(n=33)$ ED6 ( $=24)$

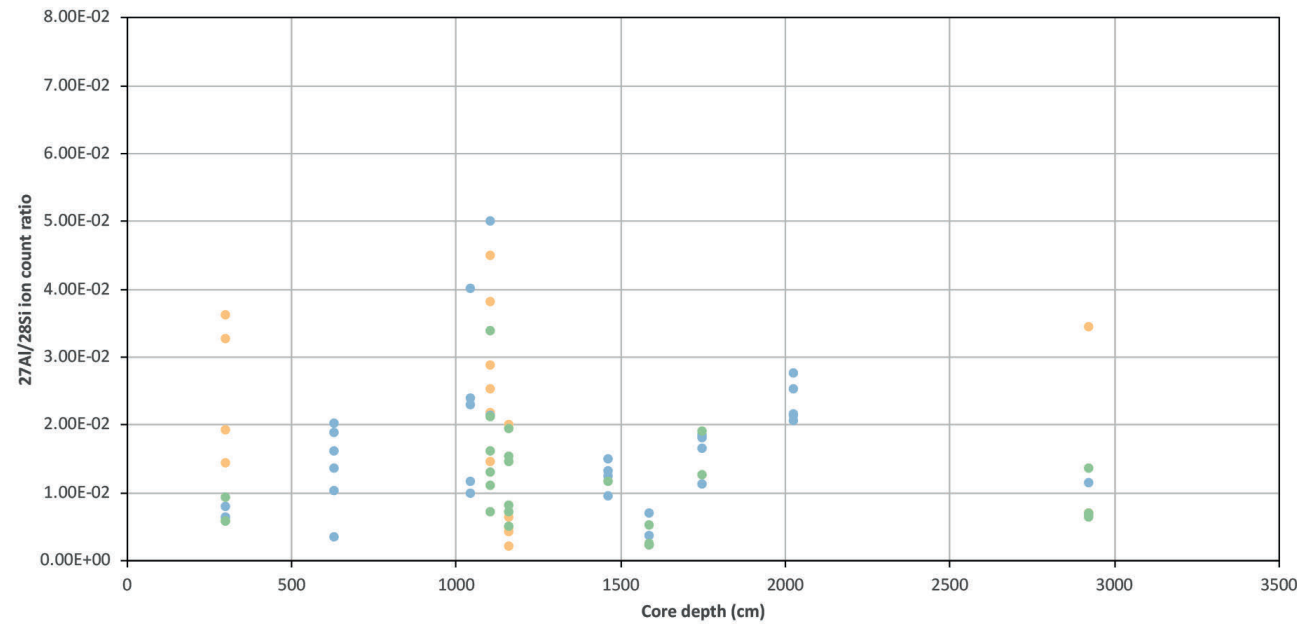

Figure S2.9: Datapoints from three different measuring cycles (ED4; ED5; ED6) after the removal of contamination hotspots, depth profile stability check, species correction and removal of outliers; $\mathrm{N}=$ 75. (a) $\mathrm{Fe} / \mathrm{Si}$ ion count ratio (b) $\mathrm{Al} / \mathrm{Si}$ ion count ratio

\section{Supplement 2.2 Statistical evaluation of 'interior vs. exterior' metal/Si ratios}

a)

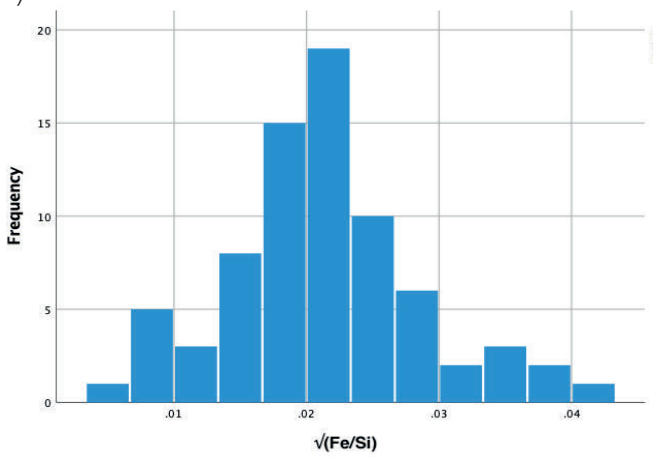

b)

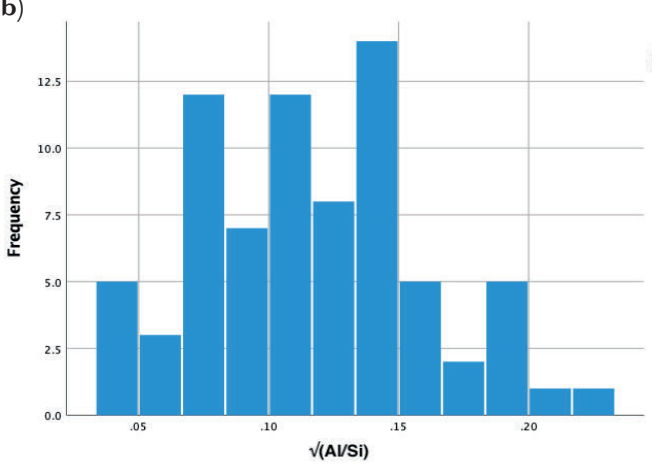

Figure S2.10: Histograms of the square-root transformed data after data processing. The ion count ratio data was square-root transformed to account for the varying intrinsic metal/Si ratios of the different downcore samples (a) Fe/Si; (b) $\mathrm{Al} / \mathrm{Si}$

Table S2.1: Shapiro-Wilk test of normality All data was shown to be normally distributed after square root transformation.

\begin{tabular}{lccc}
\hline & Statistic & df & Sig. \\
\hline SQRT(Al/Si) & .979 & 75 & .242 \\
SQRT(Fe/Si) & .975 & 75 & .139 \\
\hline
\end{tabular}


Table S2.2: Brown-Forsythe test for homoscedasticity This test was used as it is most appropriate when group sizes are unequal. The assumption was met for $\mathrm{Al} / \mathrm{Si}$ but not for $\mathrm{Fe} / \mathrm{Si}$.

\begin{tabular}{lcccc}
\hline & Levene Statistic & df & df2 & Sig. \\
\hline SQRT(Al/Si) & 1.422 & 75 & 56 & .183 \\
SQRT(Fe/Si) & 2.825 & 75 & 56 & .004 \\
\hline
\end{tabular}

Table S2.3: Two-factor ANOVA (A1/Si) The 'Exterior/Interior' factor was set as fixed and the 'Sample' factor was set as random. The measurements on the interior and the exterior were shown to be significantly different. The interaction term between sample and exterior/interior was not significant.

\begin{tabular}{|c|c|c|c|c|c|c|c|}
\hline Source & & $\begin{array}{l}\text { Type III Sum } \\
\text { of squares }\end{array}$ & df & Mean Square & $F$ & Sig. & $\omega^{2}$ \\
\hline \multirow[t]{2}{*}{ Intercept } & Hypothesis & .598 & 1 & .598 & 131.464 & .000 & 0.931 \\
\hline & Error & .044 & 9.746 & .005 & & & \\
\hline \multirow[t]{2}{*}{ Exterior vs Interior } & Hypothesis & .008 & 1 & .008 & 5.835 & .036 & 0.368 \\
\hline & Error & .014 & 10.025 & .001 & & & \\
\hline \multirow[t]{2}{*}{ Sample } & Hypothesis & .051 & 9 & .006 & 3.727 & .048 & 0.826 \\
\hline & Error & .011 & 7.059 & .002 & & & \\
\hline \multirow[t]{2}{*}{ Interaction } & Hypothesis & .012 & 8 & .001 & 1.662 & .128 & 0.192 \\
\hline & Error & .049 & 56 & .001 & & & \\
\hline
\end{tabular}

Table S2.4: Two-factor ANOVA (Fe/Si) The 'Exterior/Interior' factor was set as fixed and the 'Sample' factor was set as random. The measurements on the interior and the exterior were shown to be significantly different. The interaction term between sample and exterior/interior was also significant. The results for this test should be met with extreme caution as the homogeneity of variance assumption could not be met.

\begin{tabular}{|c|c|c|c|c|c|c|c|}
\hline Source & & $\begin{array}{l}\text { Type III Sum } \\
\text { of squares }\end{array}$ & df & $\begin{array}{l}\text { Mean } \\
\text { Square }\end{array}$ & $\mathbf{F}$ & Sig. & $\omega^{2}$ \\
\hline \multirow[t]{2}{*}{ Intercept } & Hypothesis & .019 & 1 & .019 & 123.929 & .000 & 0.930 \\
\hline & Error & .001 & 9.258 & .000 & & & \\
\hline \multirow[t]{2}{*}{ Exterior vs Interior } & Hypothesis & .000 & 1 & .000 & 7.624 & .022 & 0.460 \\
\hline & Error & .000 & 8.965 & $4.769 E-5$ & & & \\
\hline \multirow[t]{2}{*}{ Sample } & Hypothesis & .002 & 9 & .000 & 3.359 & .056 & 0.800 \\
\hline & Error & .000 & 7.534 & $5.833 E-5$ & & & \\
\hline \multirow[t]{2}{*}{ Interaction } & Hypothesis & .000 & 8 & $5.407 E-5$ & 3.406 & .003 & 0.327 \\
\hline & Error & .001 & 56 & $1.587 E-5$ & & & \\
\hline
\end{tabular}


a)

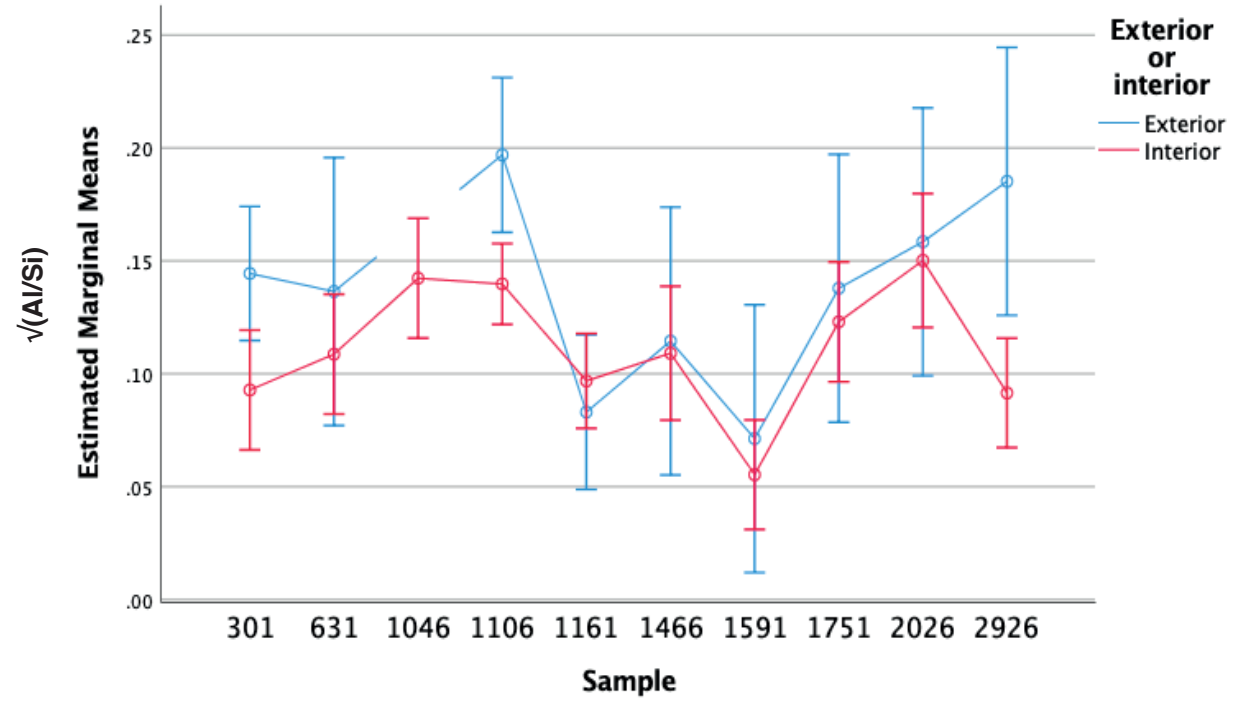

Non-estimable means are not plotted

Error bars: $95 \% \mathrm{Cl}$

b)

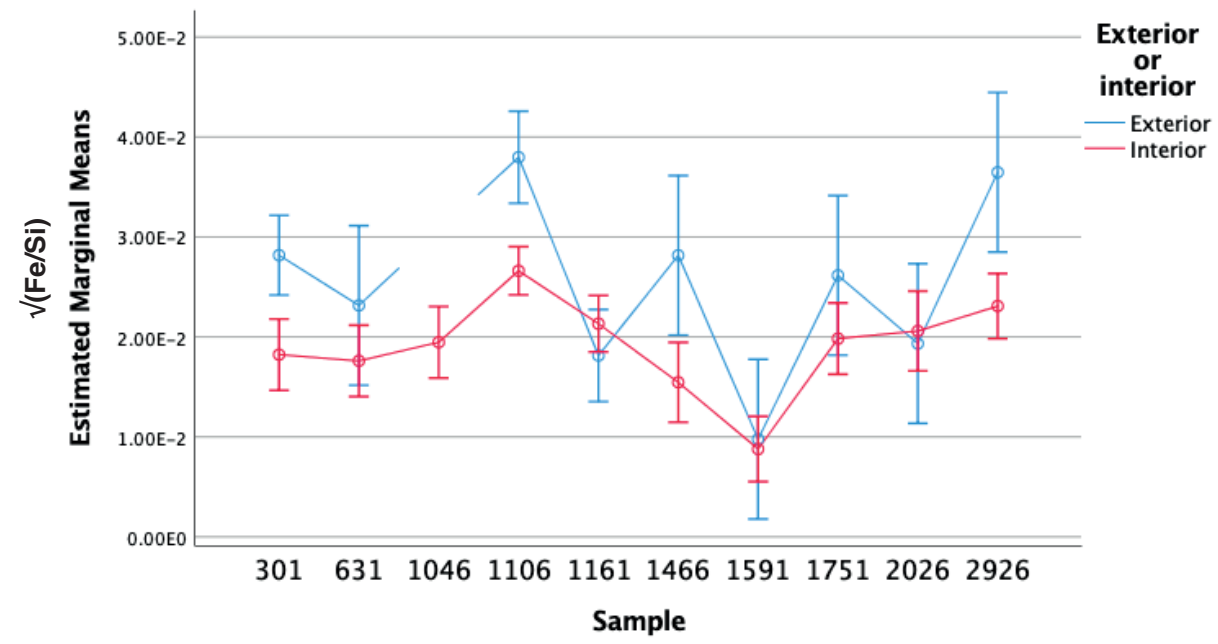

Non-estimable means are not plotted

Error bars: $95 \% \mathrm{Cl}$

Figure S2.11: Line charts showing the estimated marginal means of the square-root transformed data; (a) $\mathrm{Al} / \mathrm{Si}$; (b) $\mathrm{Fe} / \mathrm{Si}$ 
Table S2.5: Post-Hoc T-tests for 'Exterior/Interior' factor Due to lower sample counts, the Levene's test for equal variances could not be completed for most tests. Only samples 1161, 1106 and the Al/Si for sample 301 passed the test. For all others, equal variances are assumed but this is without confidence

\begin{tabular}{|c|c|c|c|c|}
\hline & Levene Statistic & $t$ & df & Sig. \\
\hline \multirow[t]{2}{*}{2926} & SQRT(Al/Si) & 4.084 & 5 & .010 \\
\hline & $S Q R T(F e / S i)$ & 5.489 & 5 & .003 \\
\hline \multirow[t]{2}{*}{2026} & $S Q R T(A l / S i)$ & -0.647 & 3 & .564 \\
\hline & $S Q R T(F e / S i)$ & 0.716 & 3 & .526 \\
\hline \multirow[t]{2}{*}{1751} & SQRT(AI/Si) & 1.953 & 4 & .123 \\
\hline & $S Q R T(\mathrm{Fe} / \mathrm{Si})$ & 0.991 & 4 & .378 \\
\hline \multirow[t]{2}{*}{1591} & SQRT(Al/Si) & 0.554 & 5 & .610 \\
\hline & $\mathrm{SQRT}(\mathrm{Fe} / \mathrm{Si})$ & 1.041 & 5 & .346 \\
\hline \multirow[t]{2}{*}{1466} & SQRT(Al/Si) & 3.588 & 3 & .037 \\
\hline & $\mathrm{SQRT}(\mathrm{Fe} / \mathrm{Si})$ & 0.464 & 3 & .675 \\
\hline \multirow[t]{2}{*}{1161} & SQRT(Al/Si) & -1.296 & 9 & .227 \\
\hline & SQRT(Fe/Si) & -0.602 & 9 & .562 \\
\hline \multirow[t]{2}{*}{1106} & SQRT(AI/Si) & 4.998 & 12 & .000 \\
\hline & $S Q R T(F e / S i)$ & 2.532 & 12 & .026 \\
\hline \multirow[t]{2}{*}{631} & SQRT(Al/Si) & 1.226 & 4 & .287 \\
\hline & $\mathrm{SQRT}(\mathrm{Fe} / \mathrm{Si})$ & 0.793 & 4 & .472 \\
\hline \multirow[t]{2}{*}{301} & SQRT(Al/Si) & 2.051 & 7 & .079 \\
\hline & $\mathrm{SQRT}(\mathrm{Fe} / \mathrm{Si})$ & 2.028 & 7 & .082 \\
\hline
\end{tabular}




\section{Supplement 2.3 The NanoSIMS record}

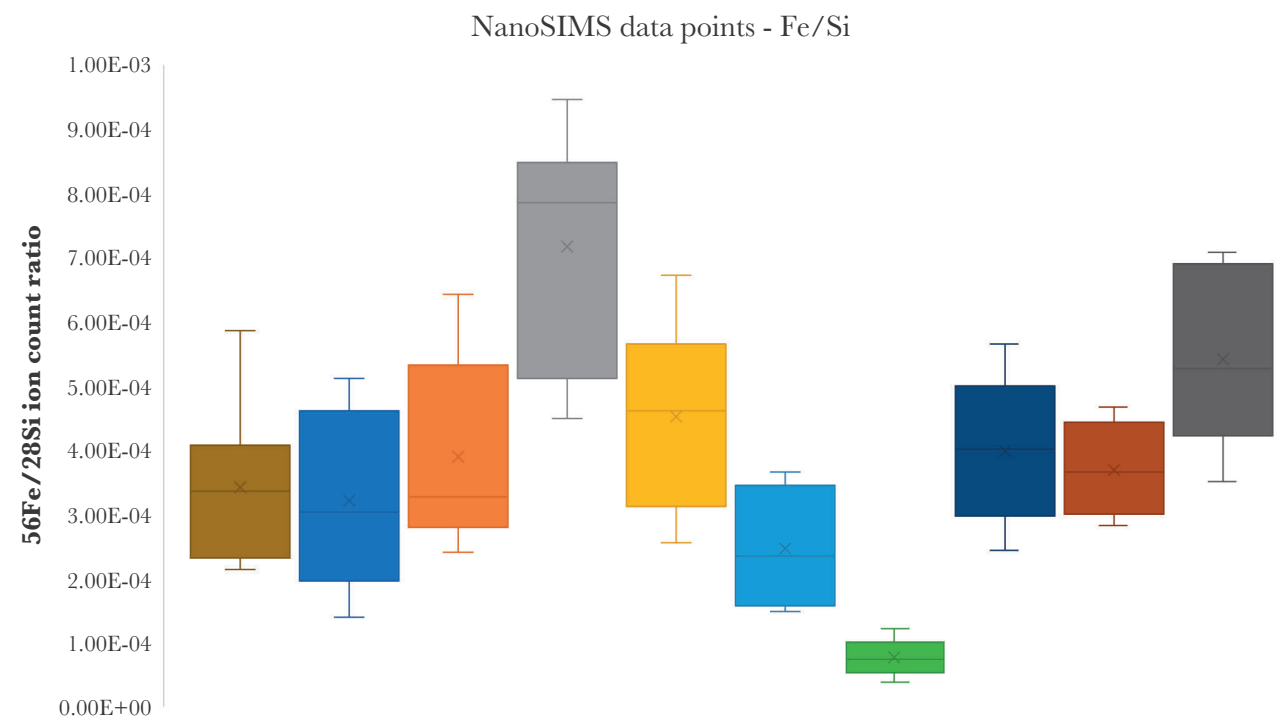

$\square 301 \square 631 \square 1046 \square 1106 \square 1161 \square 1466 \square 1591 \square 1751 \square 2026 \square 2926$

NanoSIMS data points - $\mathrm{Al} / \mathrm{Si}$

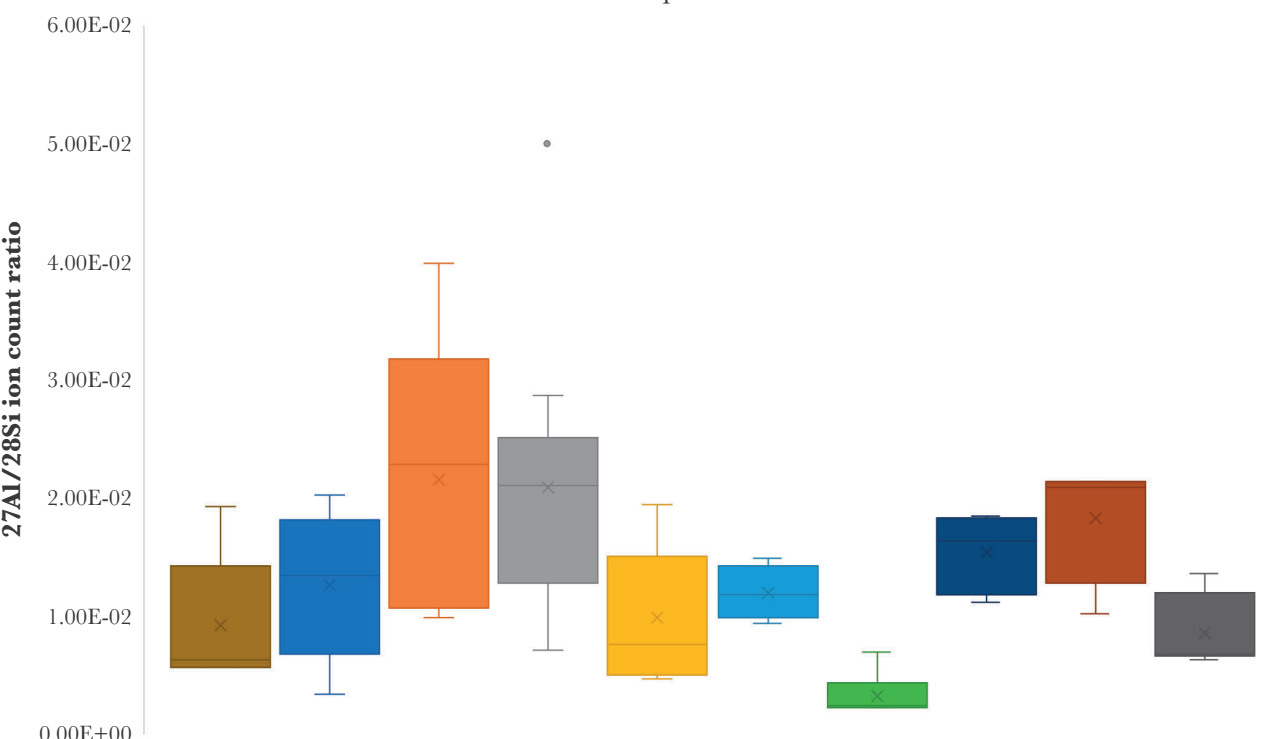

$\square 301 \square 631 \square 1046 \square 1106 \square 1161 \square 1466 \square 1591 \square 1751 \square 2026 \square 2926$

Supplementary figure S2.12: Final nanoSIMS Record Shown are the $\mathrm{Fe} / \mathrm{Si}$ and $\mathrm{Al} / \mathrm{Si}$ ion count ratios of all frustule interior nanoSIMS measurements after the data processing steps discussed in supplement 2.1. The final record has 59 datapoints, which each representing an individual frustule. Sample names in the legend represent the sample core depth. Means are represented by ' $x$ '; The two ratios do not appear to be strongly coupled $\left(\mathrm{R}^{2}=0.37\right)$ 


$$
3
$$




\title{
Chapter 3
}

\section{Mapping of organic signals in individual fossil diatom frustules with nanoSIMS and Raman spectroscopy}

\author{
Shaun P. Akse ${ }^{1}$, Gobind Das ${ }^{2 *}$, Lubos Polerecky ${ }^{1}$, Susana Agusti ${ }^{2}$ and Jack J. Middelburg ${ }^{1}$ \\ 1 Department of Earth Sciences, Utrecht University, PO Box 80021, 3508 TA Utrecht, The Netherlands \\ 2 Red Sea Research Center and Core Labs, King Abdullah University for Science and Technology, Thuwal, Saudi Arabia \\ * Correspondence to Department of Physics, Khalifa University, Abu Dhabi, P.Box-127788, UAE
}

\begin{abstract}
The application of $\delta^{15} \mathrm{~N}_{\text {diatom }}$ for determining past changes in nitrate consumption, is based on the premise that the organic matter occluded in the silica is protected from diagenesis. However, the location of the organic matter within the fossil frustule has hitherto not been identified. Here, we combine high spatial resolution imaging by nanoSIMS and Raman micro-spectroscopy to identify where the organic material is retained in the cleaned fossil diatom frustule. NanoSIMS imaging suggested that organic signals were present throughout the frustule but in higher concentrations at the pore walls. Raman measurements confirmed the heterogenous presence of organics but could (because of lower resolution) not resolve the spatial patterns observed by nanoSIMS.
\end{abstract}




\subsection{Introduction}

Diatoms, a ubiquitous group of microalgae, construct frustules made of hydrated amorphous silica that can be preserved in the sediment record. The application of diatom frustule paleoproxies has increased greatly in the last decade. The nitrogen isotope composition of diatom frustules has been proposed as a proxy for nitrogen utilization and thus surface nutrient consumption (i.e., Horn et al., 2011; Robinson et al., 2014). This proxy is based on the presence of different proteins (mainly pleuralins, silaffins and long chain polyamines) necessary for silica sequestration that are incorporated into the diatom frustule during growth (Kroger et al., 2002; Hecky et al., 1973; Kröger and Poulsen, 2008; Bridoux and Ingalls, 2010). During a period of growth, phytoplankton draw down nutrient concentrations, resulting in an increase of residual nitrate $\delta^{15} \mathrm{~N}$ due to fractionation of $\mathrm{N}$ isotopes during nutrient uptake (through Rayleigh distillation). The rise in $\delta^{15} \mathrm{~N}_{\text {nitrate }}$ with nutrient depletion is expected to be recorded in the biogenic silica (opal) produced by diatoms (De La Rocha, 2006). As such, high $\delta^{15} \mathrm{~N}_{\text {diatom }}$ indicate that a greater fraction of available nutrients (nitrate) has been consumed during the growing season (Shemesh et al., 1993).

It has been suggested that, in contrast to the bulk sediment, nitrogen encased within the diatom frustule is protected from diagenetic alteration and thus presents a more robust proxy (Shemesh et al., 1993; Sigman et al., 1999). These assumptions are based on observations such as similar relative amino acid abundances in the organic matter of fossil frustules and living diatoms (Shemesh et al., 1993), the apparent absence of diagenetic variability in frustule-bound $\mathrm{N}$ when compared to bulk sediment N content (Sigman et al., 1999), and high diatom-bound amino acid concentrations in silica-rich sediments relative to sediment traps (Ingalls et al., 2003). However, as of yet, it is unknown where exactly in the frustule this organic matter resides.

For $\delta^{15} \mathrm{~N}_{\text {diatom }}$ analysis, several precautions are generally taken to ensure that only the frustulebound nitrogen is measured, including several harsh cleaning methods to remove any loose organic matter and diagenetic alteration (Robinson et al., 2004; Horn et al., 2011b). Despite this, questions remain regarding the nature of the occluded organic matter. The isotope composition of entombed organic matter may vary, for example, due to species-specific amino acid compositions (Des Combes et al., 2008) or species-specific fractionation (Horn et al., 2011b). Possible isotopic alteration of the occluded organic matter during diagenesis remains largely unexplored, but it has been shown that the diagenetic incorporation of metals does not affect the $\mathrm{N}$ isotope values of the frustule bound organic matter (Ren et al., 2013).

This study aims to identify where in the cleaned fossil diatom frustule the organic material is retained. Our approach involved combination of nanoSIMS (nanoscale secondary ion mass spectrometry) and Raman spectroscopy, which are complementary methods suitable for highresolution imaging of signals associated with organics. NanoSIMS allows imaging of elements with a lateral resolution down to $50 \mathrm{~nm}$, but is limited by its sensitivity to the $3 \mathrm{D}$ sample topography due to the design of the ion optics. Furthermore, NanoSIMS typically probes only the upper few nm of the sample due to the low primary ion beam currents used to achieve high lateral resolution. In contrast, the Raman technique integrates signals over larger depths in the sample, but has a distinctly poorer lateral resolution ( $\mu \mathrm{m}$ scale). However, by probing of chemical bonds, Raman spectroscopy allows identification of the molecular structure of samples. By combining the data from these two techniques, we confirm the presence of organic matter in the frustules and suggest patterns of its heterogenous distribution within the frustule. 


\subsection{Materials \& Methods}

\subsubsection{Materials}

The samples for this study were obtained through collaboration with the University of Edinburgh and originate from sediment Core MD-022515. This core was retrieved from the Guaymas Basin $\left(27^{\circ} 29.01 \mathrm{~N} ; 112^{\circ} 04.46 \mathrm{~W}\right.$; $881 \mathrm{~m}$ water depth) during the MONA (Marges Ouest Nord Américaines) cruise of the R/V Marion Dufresne (International Marine Global Changes-IMAGES VIII) in June 2002 (Pichevin et al., 2012). The diatom frustules were collected from a core depth of $1161 \mathrm{~cm}$, corresponding to an age of 12450 years BP according to Pichevin et al. (2012). Cleaning of the samples was performed following published protocols (Morley et al., 2004; Hendry and Rickaby, 2008), and comprised both mechanical and chemical cleaning steps including the removal of unwanted organics with $40 \% \mathrm{H}_{2} \mathrm{O}_{2}$ at $80-90{ }^{\circ} \mathrm{C}$ (see Pichevin et al., 2014 and Hendry \& Rickaby (2008) for additional information on the performed steps).

\subsubsection{Sample preparation and selection}

To prepare the samples for nanoSIMS analysis, a needle-tip portion of the diatom frustules was pressed onto an ultra-clean indium foil (99.99\% indium; Alfa Aesar; 0.05mm thick) attached to an aluminum stub. The sample was subsequently coated with a $12 \mathrm{~nm}$ thick Au layer using a sputter coater (JOEL JFG-2300HR high resolution fine coater, JEOL FG-TM20 thickness controller). The sample was then imaged with a table-top SEM (JEOL JCM-6000PLUS NeoScope Benchtop SEM) operating at a $15-\mathrm{kV}$ accelerating voltage to identify specimens suitable for further analysis (individual frustules with minimal 3D topography).

\subsubsection{NanoSIMS imaging}

Nanoscale secondary ion mass spectrometry was performed with the nanoSIMS 50L instrument (Cameca) operated at Utrecht University. Electron multiplier detectors were set using a standard (SPI Supplies, 02757-AB 59 Metals \& Minerals Standard) to enable the detection of secondary ions ${ }^{12} \mathrm{C}^{-},{ }^{16} \mathrm{O}^{-},{ }^{12} \mathrm{C}^{14} \mathrm{~N}^{-},{ }^{28} \mathrm{Si}^{-},{ }^{31} \mathrm{P}^{-}$and ${ }^{32} \mathrm{~S}^{-}$with the ${ }^{133} \mathrm{Cs}^{+}$primary ion beam. Prior to the analysis of each target frustule, an area slightly larger than the region of interest was pre-sputtered with the primary ion beam (FGo current 20pA, $\sim 360$ s) to remove the gold coating and reach stable secondary ion yields. After pre-sputtering, the instrument was tuned with diaphragm and slit settings of D1-3, ES-3, AS-2 and EnS-1. Analysis was carried out in the imaging mode by rastering a high-energy ${ }^{133} \mathrm{Cs}^{+}$ion beam (16 keV, 2pA FCo, nominal beamsize 50-150 nm; electron gun enabled) over a target area on the frustule (between $4 \mu \mathrm{m} \times 4 \mu \mathrm{m}$ and $55 \mu \mathrm{m} \times 55 \mu \mathrm{m}$ in size) and measuring the sputtered secondary ions at a resolution of either 128x128 or $256 \times 256$ pixels and with a dwell time of $1 \mathrm{~ms} /$ pixel. Target areas were measured multiple times (typically over 400-600 planes) to increase signals and provide depth resolution. The combination of the primary ion current, dwell time and number of measured planes did not allow measurements through the entire thickness of the frustule. Data processing and analysis were done with an updated version of the Look@ nanoSIMS software (Polerecky et al., 2012).

Initial measurements targeted frustules of different species of centric and pennate diatoms. However, pennate diatom frustules proved difficult to measure with the NanoSIMS due to their pronounced 3D topography. Therefore, only data obtained from centric diatom frustules, including the valve interior and exterior, are presented. 


\subsubsection{Raman spectroscopy and imaging}

Raman measurements were performed with the Horiba LabRam spectrometer at the King Abdullah University for Science and Technology. The sample was excited with a $473 \mathrm{~nm}$ laser line in the back-scattering configuration through an 100X objective. Using a grating with 1800 grooves/ $\mathrm{mm}$, Raman spectra were collected in the range of $150-3300 \mathrm{~cm}^{-1}$ with a spectral resolution of $1.2 \mathrm{~cm}^{-1}$. Initially, the interior of an individual frustule was analysed in three spots to identify the primary signals present. This was followed by imaging analysis of the interiors and exteriors of several frustules with the step size varying between $0.5-2 \mu \mathrm{m}$ and the size of the imaged areas varying between $8 \mu \mathrm{m} \times 8 \mu \mathrm{m}$ and $15 \mu \mathrm{m} \times 15 \mu \mathrm{m}$. During imaging with the lower step size $(0.5 \mu \mathrm{m}$ and $1 \mu \mathrm{m}$ ) only the lower frequency range (up to $1700 \mathrm{~cm}^{-1}$ ) was recorded, whereas the larger step size imaging also covered higher frequencies (around $3000 \mathrm{~cm}^{-1}$ ) to include a prominent organic peak between 2826-3021 cm-1. Data analyses were performed using the manufacturer's software LabSpec (version 6).

\subsection{Results}

\subsubsection{Identification of organics by Raman spectroscopy}

The presence of organics in the silica matrix of a cleaned fossil diatom frustule was confirmed by Raman measurements in multiple spots on the frustule (Fig. 3.1; Table 3.1). Besides Raman peaks associated with tetrahedral Si-O-Si, Si-O-Si network, Si-OH stretching and aluminium, there was clear evidence for organics at wavenumbers around 1425 and $2910 \mathrm{~cm}^{-1}$.

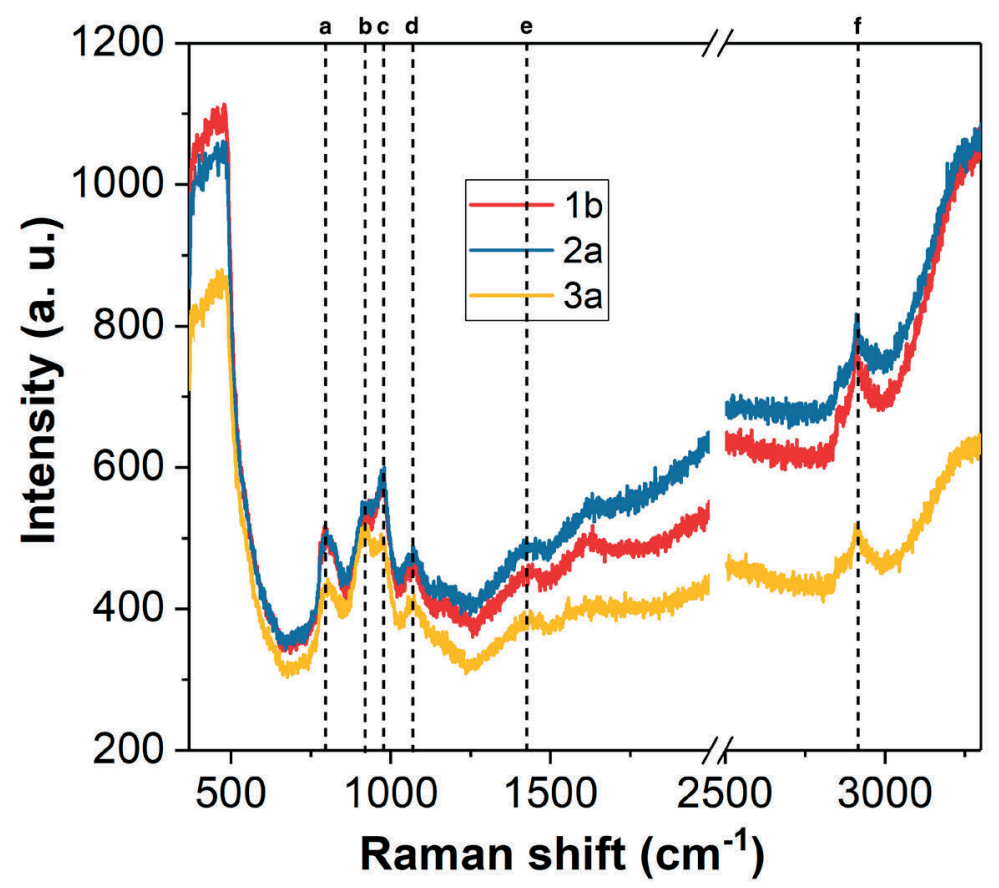

Figure 3.1: Single point Raman spectra obtained from a cleaned fossil frustule of a centric diatom. Shown are spectra measured at three different locations within the same frustule. Peak assignments are summarized in Table 1. 
Table 3.1: Raman band assignments

\begin{tabular}{ll}
\hline Band position $\left(\mathrm{cm}^{-1}\right)$ & Band assignments \\
\hline a) $768-848$ & Inter tetrahedral Si-O-Si \\
b) $870-952$ & Aluminium derivatives \\
c) $945-1010$ & Si-OH stretching \\
d) $1020-1134$ & Si-O-Si network \\
e) $1364-1438$ & SC-Hx \\
f) $2826-3021$ & $\mathrm{VC}-\mathrm{Hx}$ \\
\hline
\end{tabular}

Identification was based on (Terpstra et al., 1990; McMillan, 1984; Kammer et al., 2010; De Tommasi et al., 2018 ).

\subsubsection{NanoSIMS imaging of frustules}

After establishing the presence of organics with single point Raman analyses, the elemental distributions were imaged with nanoSIMS. Secondary ions $\mathrm{CN}^{-}$(detected as ${ }^{12} \mathrm{C}^{14} \mathrm{~N}^{-}$) and $\mathrm{O}^{-}$were used as a proxy for organics and silica, respectively. Generally, $\mathrm{CN}^{-}$ions were detected throughout the frustule but showed significantly higher counts in ring-like structures around the pores and in hotspots scattered across the frustule or near the edges of the frustule (Fig. 3.2b and 3.2f). The patterns were similar for both the frustule interior and exterior, although the surface morphology differed markedly between the two sides (compare Fig. 3.2a-b and 3.2e-f). Similar patterns were also observed for other elements associated with organics including $\mathrm{C}$ and S (Fig. 3.2c-d and 3.2gh). High-resolution SEM images revealed that, on the frustule interior, the ring-like structures were associated with a clear thickening of the silica matrix around the pore (see inset in Fig. 3.2a). The difference in contrast in the SEM images suggested that the silica matrix in these structures was different from the rest of the matrix, consistent with the differences observed by NanoSIMS.

\subsubsection{Mapping of organics by Raman spectroscopy}

Although the signal around $2910 \mathrm{~cm}^{-1}$ had a stronger presence in the Raman spectra (Fig. 3.1), subsequent mapping focused on the peak at $1425 \mathrm{~cm}^{-1}$, which allowed reduction in the frequency range and thus improved lateral resolution. Images obtained by Raman measurements showed pronounced heterogeneity in the signals corresponding to both the silica matrix and organics (Fig. 3.3). The intensity of the peaks corresponding to $\mathrm{Si}-\mathrm{OH}$ and $\mathrm{Si}-\mathrm{O}-\mathrm{Si}$ appeared less variable than the intensity of the $\delta \mathrm{C}-\mathrm{Hx}$ peak. Images of the peak ratio $\delta \mathrm{C}-\mathrm{Hx} / \mathrm{Si}-\mathrm{O}-\mathrm{Si}$ were heterogeneous (Fig. $3.3 \mathrm{e})$, but even the lowest step size $(0.5 \mu \mathrm{m}$ step size $)$ did not resolve the spatial patterns detected by NanoSIMS (compare Fig. 3.2 and 3.3). Raman images of the frustule interior and exterior appeared similar (Fig. 3.3). 

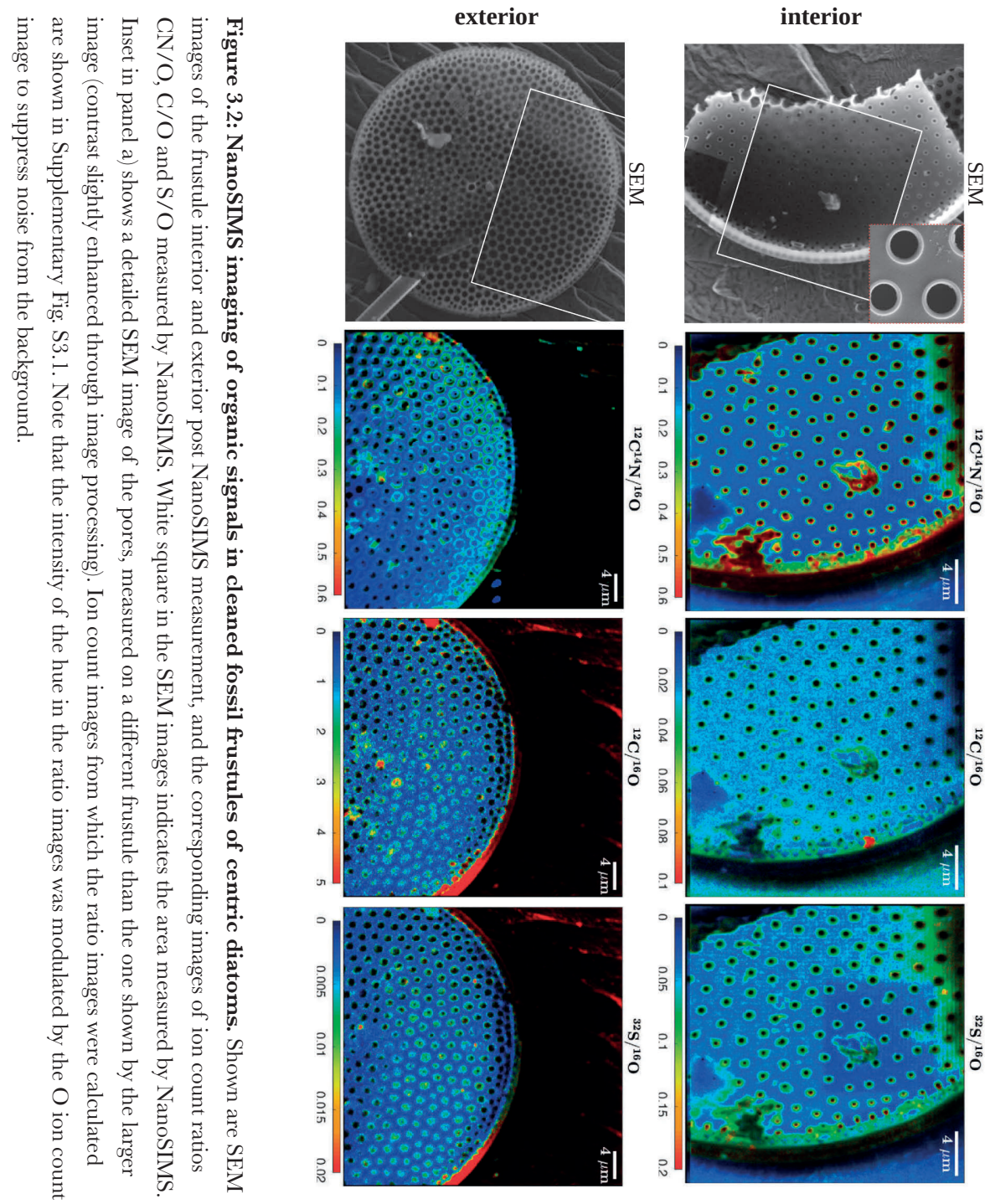


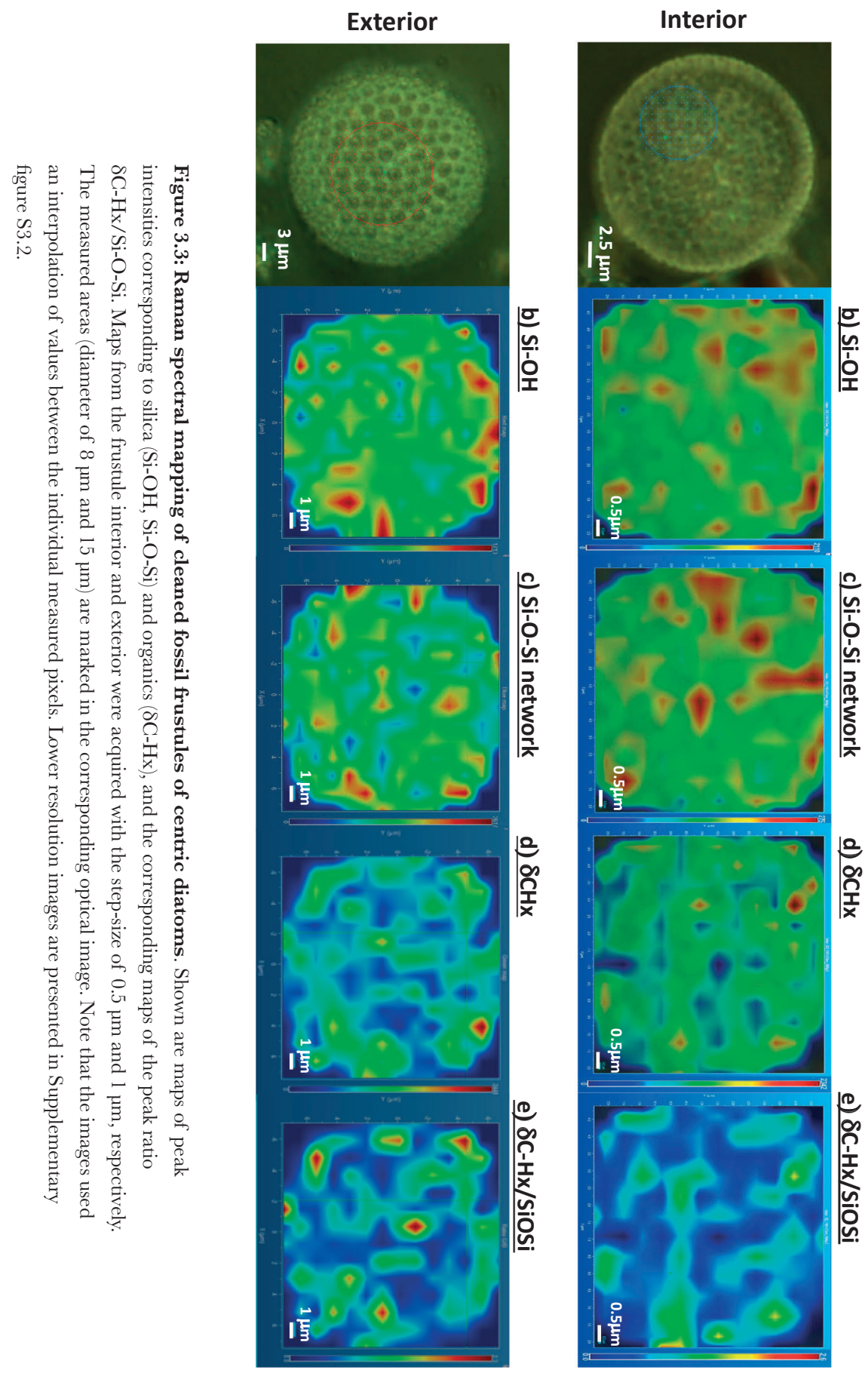




\subsection{Discussion}

By analysing cleaned fossil diatom frustules with two separate techniques, the presence of organics tightly bound to the silica matrix was confirmed. Furthermore, the chemical mapping capabilities of the techniques made it possible to resolve, to a variable degree, the patterns in the distribution of organics in the frustule. This revealed a heterogeneity and it is suggested that this is due to enrichments around the pores.

\subsubsection{Comparison of the nanoSIMS and Raman measurements}

NanoSIMS images revealed intensification of organic signals at the pore edges of the (centric) diatom frustules (Fig. 3.2). The GN signal in particular, but also the signals of $\mathrm{C}$ and $\mathrm{S}$, were clearly higher around the pores, and this spatial pattern remained as more material was sputtered away during the measurement. On the frustule interior, this signal enrichment is likely linked to the thickening of the silica matrix, which was often observed at the pore boundaries (Fig. 3.2a). As similar patterns were also observed on the frustule exterior, it is likely that the entire pore wall is marked by an increased organic presence (Fig. 3.4).

However, nanoSIMS measurements are known to be sensitive to 3D topography of the sample surface, leading for instance to higher secondary count rates at or around the edges of the studied sample. Thus, a degree of caution is required when interpreting nanoSIMS data. Although we made a thorough and critical assessment of our data, including the analysis of depth profiles of ion counts and ion count ratios, we cannot exclude that these "edge effects" played a role during the NanoSIMS measurements. Other techniques without this caveat but with a comparable sensitivity and spatial resolution are needed to verify our results.

The Raman spectroscopy method was chosen as a possible candidate for resolving this issue. This technique is also surface-specific, but it integrates the signal over a larger depth interval $(\sim \mu \mathrm{m})$ and is thus less likely affected by the edge effects. On the one hand, Raman measurements confirmed the presence of organic signals in cleaned fossil frustules, and indicated that they are heterogeneously distributed within the silica matrix. On the other hand, their lateral resolution (down to $0.5 \mu \mathrm{m}$ ) was insufficient to confirm or reject the spatial patterns observed by nanoSIMS.

\subsubsection{Implications}

An increased presence of organics in the pore walls (Fig. 3.4) may be due to a combination of the biomineralization process and the exchange of nutrients, through the pores, between the diatom cell and the surrounding water. During silicification, a protein template forms the basis onto which the silica frame is secreted (Thamatrakoln and Hildebrand, 2008; Hildebrand et al., 2018). An imaging study of the insoluble organic matrix (Tesson and Hildebrand, 2013) found that the pores of a diatom valve can be occluded by this material. Based on these observations, it is hypothesised that, during growth, the pores are not open channels but are instead lined or completely occluded by the organic material. Remains of this material embedded in the silica may be the source of the observed patterns and the carrier for proxies such as $\delta^{15} \mathrm{~N}$. 
a)

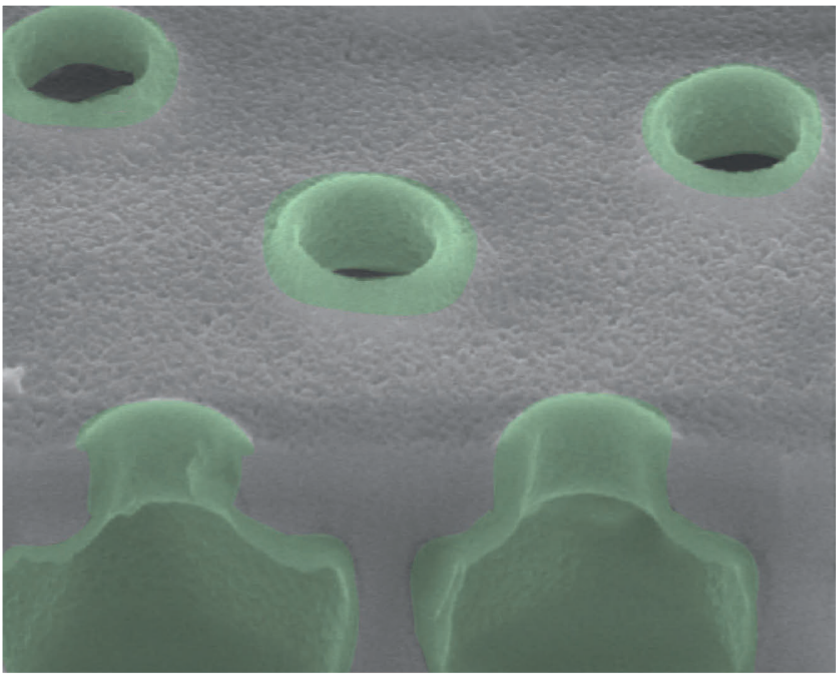

b)

Silica walls with

low organic signals

Xx

Silica walls with

high organic signals

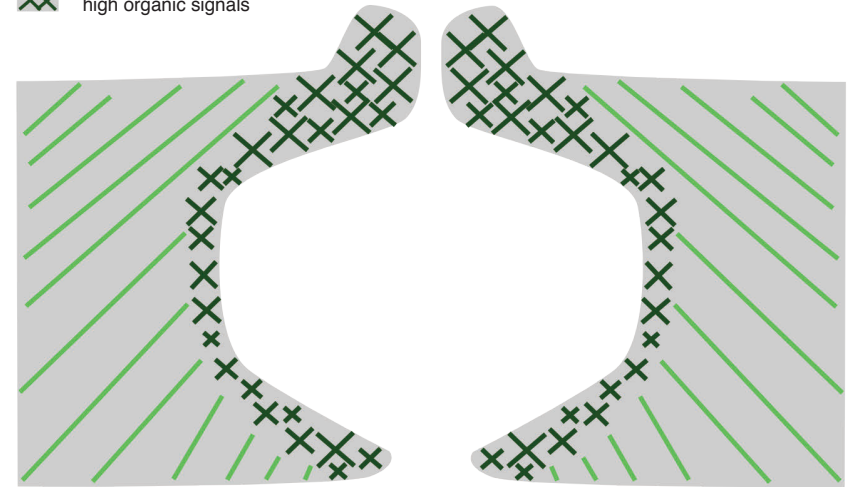

Figure 3.4: Conceptual schematic illustrating the proposed distribution of organics in fossil frustules of centric diatoms. Shown is (a) a high-resolution SEM image of a centric diatom frustule, cut with a focused ion beam (FEI Helios Nanolab G3), and (b) a conceptual diagram of the pore cross-section. Organic signals are present throughout the silica (grey/striped) but higher concentrations are present at the pore walls (green/crosses).

\subsection{Conclusions}

With a combination of two high resolution imaging techniques we were able, for the first time, to image the presence and distribution of organic signals inside the silica matrix of individual fossil diatom frustules. NanoSIMS imaging suggested that organic signals were present throughout the frustule but in higher concentrations at the pore walls. Raman measurements confirmed the presence of organics but could not resolve the spatial patterns observed by nanoSIMS. Additional research is needed to confirm and better understand the distribution of organics within the frustule of fossil diatoms. 
Supplementary figures

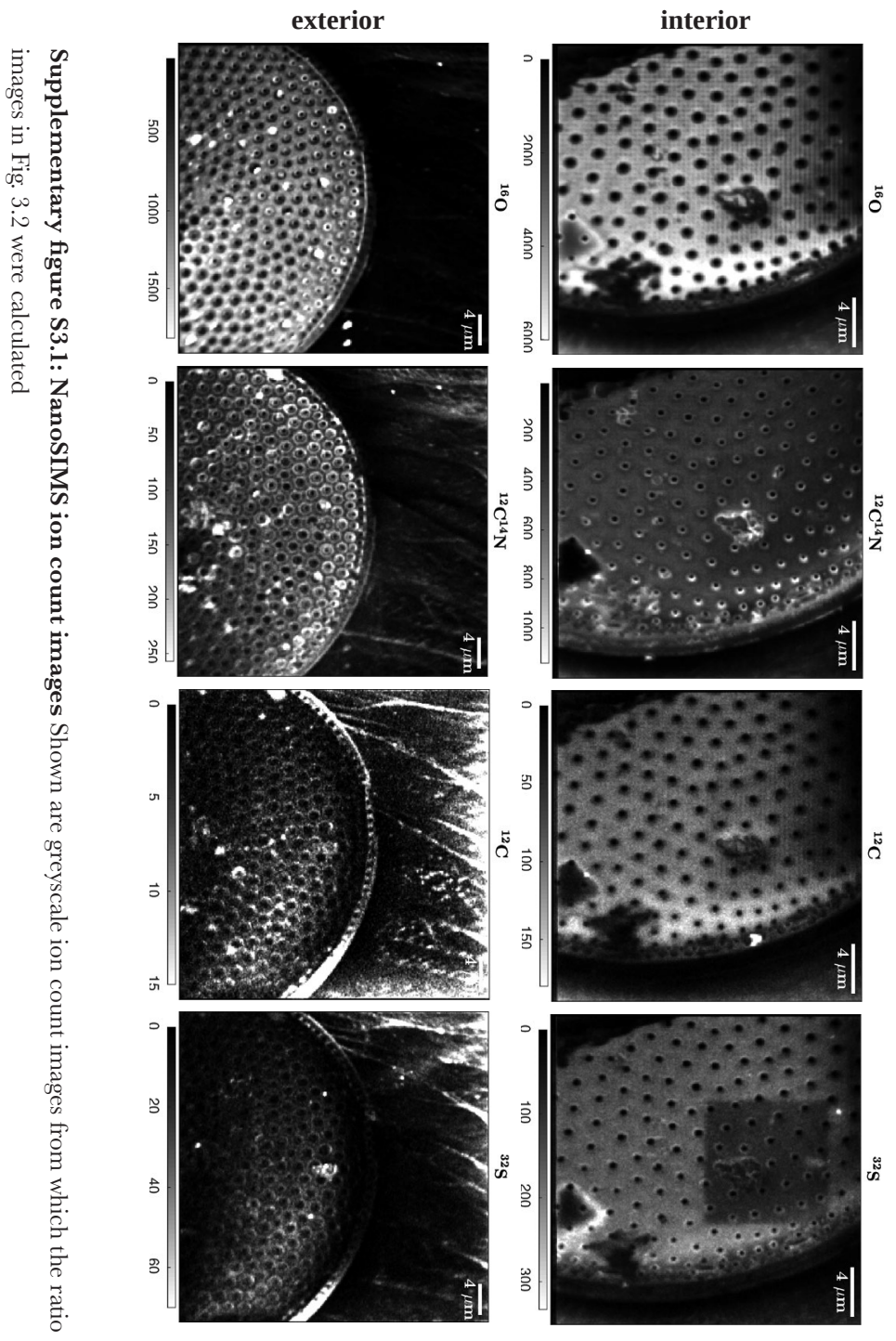




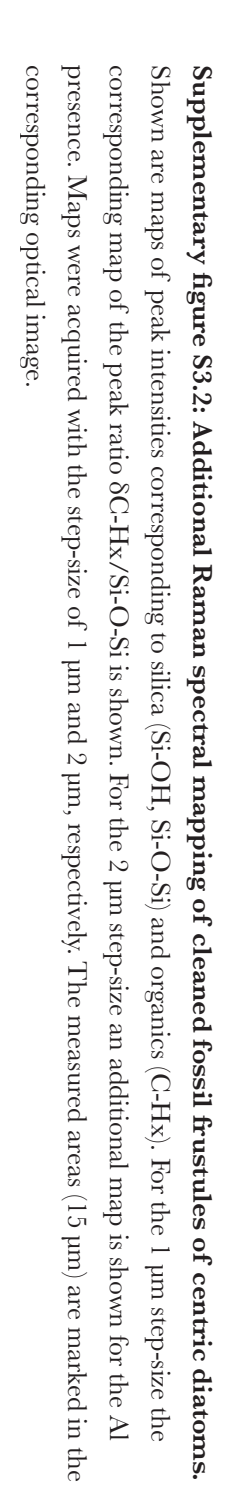

Step: $2 \mu \mathrm{m}$
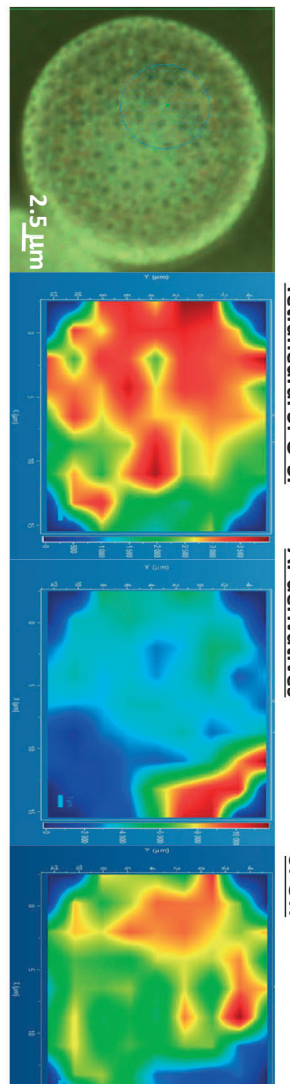

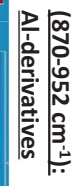
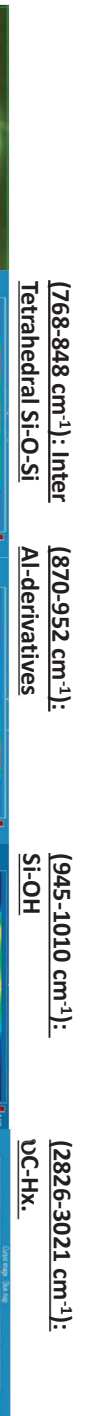

Step: $1 \mu \mathrm{m}$

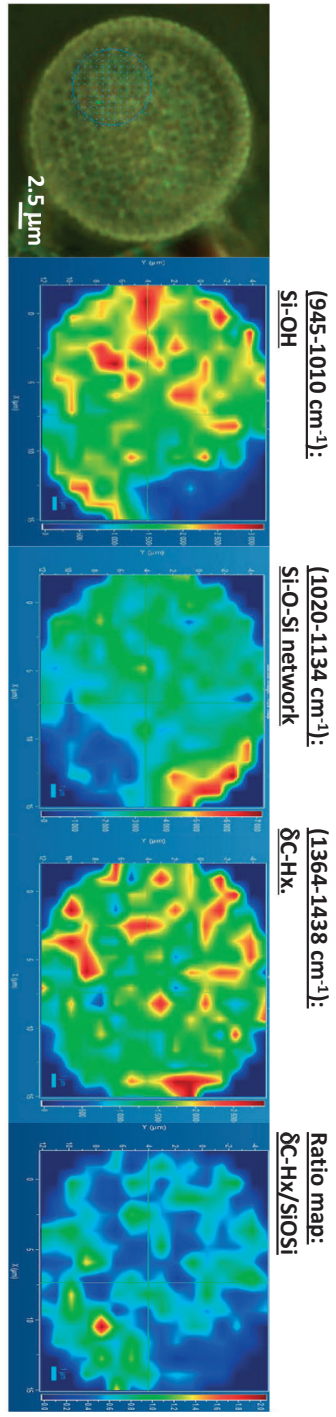




$$
4
$$




\title{
Chapter 4
}

\section{Rapid post-mortem oxygen isotope exchange in biogenic silica}

\author{
Shaun P. Akse, JackJ. Middelburg, Helen E. King and Lubos Polerecky \\ Department of Earth Sciences, Utrecht University, PO Box 80021, 3508 TA Utrecht, The Netherlands
}

\begin{abstract}
The oxygen isotope composition of diatom frustules has been the subject of study because it is thought that, similar to the $\delta^{18} \mathrm{O}_{\text {foraminifera }}$ the $\delta^{18} \mathrm{O}_{\text {diatom }}$ reflects the isotopic composition of the ambient seawater. However, significant concerns exist surrounding the degree of post-mortem diagenetic overprinting that might occur. Here, we study diagenetic overprinting of the $\delta^{18} \mathrm{O}$ signal in various forms of silica by incubating the tests in ${ }^{18} \mathrm{O}$-enriched seawater and analysing them with nanoSIMS and Raman micro-spectroscopy. The nanoSIMS results show that, on timescales similar to that of the sinking diatom detritus in marine systems (days to weeks), the $\delta^{18} \mathrm{O}$ signal of both diatom frustules and sponge spicules can be homogenously overprinted. In contrast, crystalline silica does not show any indications of ${ }^{18} \mathrm{O}$-enrichment, most likely due to the absence of silanol (Si$\mathrm{OH})$ groups as observed in Raman measurements. Together these results confirm that the silanols can be diagenetically overprinted on short timescales, and suggest that the rest of the silica structure is also susceptible. The final measured $\delta^{18} \mathrm{O}_{\mathrm{bSiO} 2}$ will therefore, most likely, represent a mixed signal from the original growing stage, water-column settling, sediment-water interface, and sediment pore waters.
\end{abstract}




\subsection{Introduction}

Studying changes in the isotopic composition of oxygen in past waters is considered an important paleoceanographic tool, as these changes reflect local as well as global changes in characteristic features of seawater, such as temperature, changes in global ice volume and other regional processes (e.g., Rohling \& Cook, 1999; Swann and Leng, 2009). Measuring $\delta^{18} \mathrm{O}$ in foraminiferal shells is a commonly used method to track changes in the $\delta^{18} \mathrm{O}$ of ambient water during growth (e.g., Zachos et al., 2001). Unfortunately, foraminifera and other carbonate remains are not always well preserved on the ocean floor, impeding our knowledge of paleoceanographic conditions in the geological past. Therefore, in areas where carbonates are absent, other forms of biogenic material are required to study the changes in seawater $\delta^{18} \mathrm{O}$ over time. One material that could help fill the gaps in the record is biogenic silica (opal), which is predominantly represented in the sedimentary record by diatom frustules and sponge spicules.

As all silicates, diatom silica is composed mainly of interlinked silica $\left(\mathrm{SiO}_{4}\right)$ tetrahedrons. These units connect via bridging oxygen atoms forming siloxane (- $\left.\mathrm{Si}-\mathrm{O}-\mathrm{Si}^{-}\right)$bonds (Zhuravlev, 2000). Formation of biogenic silica occurs via condensation reactions where two $\mathrm{Si}-\mathrm{OH}$ groups on the silicic acid units condense to form $\mathrm{SiO}_{2}+\mathrm{H}_{2} \mathrm{O}$. The $\delta^{18} \mathrm{O}$ of the resulting biogenic $\mathrm{SiO}_{2}$ is assumed to reflect the $\delta^{18} \mathrm{O}$ of the water in which it formed. Surrounding the tetrahedrally bonded - Si-O-Si layer, a less dense hydrous layer consisting of - Si-OH groups (silanols) exists (Gendron-Badou et al., 2003). Silanol groups can be either present on the surface of the frustule (external) or trapped in the porous structure as internal silanols (Loucaides et al., 2010; Zhuravlev, 2000). Moreover, the oxygen atom in these silanol groups is thought to continuously exchange with the oxygen atom in the surrounding water molecules (Labeyrie and Juillet, 1982; Frölich, 1989). The question remains whether both external and internal silanols are equally affected by secondary oxygen signal overprinting, and whether this has consequences for the tetrahedrally bonded oxygen. If this were the case, $\delta^{18} \mathrm{O}_{\text {diatom }}$ values would most likely be similar to those in the ambient water during biosynthesis but might start to deviate from this signal post-mortem.

Siliceous sponges present another form of biogenic silica found in the (paleo)oceans. These sponges are commonly represented in the sediments by their spicules (structural elements which support the skeleton of the animals), originating predominantly from the classes Demospongiae and Hexactinellida. Sponge spicules have an amorphous silica matrix similar to that of diatom frustules (Sandford, 2003) but differ in their formation processes as well as their physical characteristics. While diatom frustules are known for their large structural pores, spicules are often observed to have concentrically layered, needle-like structures with a single hollow central core (axial canal) (Müller et al., 2007). Additionally, the two groups of organisms represent different environmental conditions as diatoms live predominantly in the surface waters, while sponges are benthic organisms and thus live in the bottom waters. This combination of similarities and differences provides the potential to reconstruct not only surface and bottom water $\delta^{18} \mathrm{O}$, but also vertical gradients in $\delta^{18} \mathrm{O}$.

In recent decades, the application of oxygen isotopes in fossil diatom frustules has become an increasingly popular tool for reconstructing past climate and environmental changes, both in lacustrine and marine settings (see Leng and Barker, 2006; Swann et al., 2006; Swann and Leng, 2009). Despite increasing interest in this proxy, the presence of hydroxyl groups in the silica matrix and their susceptibility to diagenetic overprinting remain a point of concern. These concerns are strengthened by observed differences in $\delta^{18} \mathrm{O}_{\text {diatom }}$ between living diatoms and diatoms taken from deep-water traps and surface sediments. This disparity is particularly evident in the vast array of calculated silica-water fractionation factors (Fresh/recent: Brandriss et al., 1998; Dodd and Sharp 
2010; Sedimentary: e.g., Juillet-Leclerc and Labeyrie, 1987; Matheney and Knauth, 1989; Shemesh et al., 1992). Finding the source of this variability is essential for the future of this proxy, and studying the variability on a frustule level may aid this search.

Meanwhile, the use of oxygen isotope analysis in spicules is rare, as studies have suggested that the fractionation between sponge spicules and seawater is not systematically reliable, despite some studies showing clear trends in $\delta^{18} \mathrm{O}$ (Matheney and Knauth, 1989; Matteuzzo et al., 2013; Snelling et al., 2014). Instead, the study of sponge spicules as a paleoproxy has focused on silicon isotopes (e.g., Hendry et al. 2010; Hendry and Robinson, 2012).

With this knowledge, it becomes clear that, in order to analyse the potential of $\delta^{18} \mathrm{O}$ in biogenic silica as a paleoproxy tool, the driving mechanisms behind the observed variability need to be studied further. Recent studies regarding post-mortem diagenesis in diatom frustules have focused predominantly on diagenesis occurring in the sediments, assuming that no exchange of oxygen occurs during the settling of diatom detritus through the water column. This assumption is based on the presence of an organic membrane surrounding the frustule which protects it from dissolution (Bidle and Azam, 1999; Bidle et al., 2003) and appears to inhibit post-mortem changes in $\delta^{18} \mathrm{O}$ (Moschen et al., 2006). In the sediments, several processes may influence the hydroxyl groups and may thus induce isotopic re-equilibration. A study on dissolution effects in sedimentary frustules suggests that small changes in $\delta^{18} \mathrm{O}_{\text {diatom }}$ can be observed in a state of extreme dissolution (Smith et al., 2016). Other proposed processes are the precipitation of secondary silica and addition of silanol groups, and the condensation of silica (Schmidt et al., 2001; Dodd et al., 2012, 2017; Moschen et al., 2006). The effect of silica condensation on $\delta^{18} \mathrm{O}$ was studied by ageing cultured diatoms in a setting mimicking seafloor/sedimentary conditions (Dodd et al., 2017), which revealed rapid and significant changes in $\delta^{18} \mathrm{O}$ coinciding with a reduction in the total silanol abundance. Moreover, the silica-water fractionation relationship factor in the experimentally aged samples approached equilibrium quartz-water fractionation. Because of this, it was suggested that silicawater fractionation relationships derived from coretops/shallow sedimentary silica may represent a mixed signal from growth $\delta^{18} \mathrm{O}$ values and diagenetic hydroxylation. Furthermore, it was suggested that, despite the decreasing reactivity, this process is likely to continue for about $10^{4}-10^{6}$ years, possibly resulting in complete isotopic re-equilibration of the oxygen isotopes (Dodd et al., 2017).

To reduce the role that exchangeable oxygen in silanol groups can have in determining the $\delta^{18} \mathrm{O}_{\text {diatom, }}$, several analytical methods have been developed. Two main methods have been established and compared in an inter-laboratory study: the controlled isotope exchange (CIE) method and the stepwise fluorination (SWF) method (Chapligin et al., 2011). Both analytical methods were shown to produce comparable and reproducible results demonstrating that the observed mismatch is not due to the analysis method. Recent studies, however, suggest that sample preparation and laboratory protocols can have significant effects on the measured $\delta^{18} \mathrm{O}_{\text {diatom }}$ (Menicucci et al., 2017; Tyler et al., 2017), reviving concerns about the effectivity of the current methods in removing post-mortem $\delta^{18} \mathrm{O}_{\text {diatom }}$ signals.

In this study, we challenge the assumption that water-column diagenesis plays a marginal role in controlling $\delta^{18} \mathrm{O}_{\mathrm{bSiO} 2}$. A nanoSIMS study of post-mortem ${ }^{18} \mathrm{O}$ enrichment in fresh diatom detritus and siliceous sponge spicules incubated in ${ }^{18} \mathrm{O}$-enriched seawater reveals a rapid and homogenous signal overprinting, which is absent in crystalline silica. When combining the imaging data from the nanoSIMS with structural data from Raman analysis, the results suggest that oxygen exchange does not only occur in the readily available silanol groups but also in the denser -Si-O-Si- matrix of biogenic amorphous silica. 


\subsection{Materials \& Methods}

\subsubsection{Materials}

Two separate diatom batches were cultured (Thalassiosira pseudonana and Thalassiosira weisflogii) at the Royal Netherlands Institute for Sea Research (NIOZ) by using an f/2 medium. The culture collection of the NIOZ provided the T. weisflogii strain (CGAP 1085/18) while the T. pseudonana strain (CCAP 1085/12) was acquired at the Culture Collection of Algae and Protozoans (CGAP) in Scotland. Cultures were grown in $300 \mathrm{~cm}^{3}$ tissue culture flasks at a temperature of $20^{\circ} \mathrm{C}$ under a 16/8 light/dark cycle. A total of $2 \mathrm{~L}$ of medium per diatom species was produced and split into $500 \mathrm{~mL}$ containers for harvesting. After harvesting, the diatoms were killed by freeze-drying (Telstar LyoQuest) and stored at $-20^{\circ} \mathrm{C}$. An aliquot of each $500 \mathrm{~mL}$ container was taken for cell counts determination using flow cytometry (data not shown). Before use, frozen dry sample were diluted with $50 \mathrm{ml}$ of MilliQ $\left(18.2 \mathrm{M} \Omega \cdot \mathrm{cm}\right.$ at $\left.25^{\circ} \mathrm{C}\right)$.

The sponge spicule sample (Vazella pourtalesi) was obtained from M. C. Bart from the Universiteit van Amsterdam. The Hexactinellida class sponge specimens were collected attached to their rocky substrate by the ROPOS ROV at $300 \mathrm{~m}$ depth during the Hudson cruise 2016-019 (September 2016) at the Emerald Basin (43059'49.0” N, 6246’15.7” W).

The quartz crystals were obtained from A. Roepert from Utrecht University. The crystals were collected near Al Hajar, Oman $\left(23.340274^{\circ} \mathrm{N}, 58.565833^{\circ} \mathrm{E}\right)$ from a quartz vein permeating a Late Proterozoic phyllite. Age of the crystal has not been determined. The sand grains used in this study originate from standard construction-grade sand.

Fossil frustules from Chapter 5 of this thesis are also discussed in this manuscript. These originate from the 40-60 $\mu \mathrm{m}$ size fraction of a diatomaceous clay after a 2-week incubation with ${ }^{18} \mathrm{O}$-enriched seawater.

\subsubsection{Incubation experiments}

Two types of incubation experiments were performed in this study. Most experiments had multiple time-points to study the exchange dynamics of different materials. One experiment had only one time-point but was performed at two temperatures.

Similar set-ups were used for the incubation experiments with the biogenic silica (diatom frustules and sponge spicules) and the crystalline silica (quartz crystals and sand grains) samples. Incubations were done in $1.5 \mathrm{~mL}$ Eppendorf tubes containing $1.1 \mathrm{~mL}$ of water. The ${ }^{18} \mathrm{O}$-labeled incubation used water prepared by mixing $0.1 \mathrm{~mL}$ of $97 \% \mathrm{H}_{2}{ }^{18} \mathrm{O}$ (Sigma-Aldrich) with $1 \mathrm{~mL}$ of unlabeled natural seawater, resulting in $9 \%{ }^{18} \mathrm{O}$ atom fraction in the incubation water. Control incubations only used unlabeled natural seawater. At the end, the sample material was added to each separate tube. In a climate-controlled room, the Eppendorf tubes were put into Greiner tubes and placed in a horizontal position on a roller-table to keep the water moving and the material in suspension. Additional experiment-specific details are given below.

For half of the diatom ${ }^{18} \mathrm{O}$-labeled incubations, the seawater was autoclaved before the ${ }^{18} \mathrm{O}$-label was added, whereas for the other half the ${ }^{18} \mathrm{O}$-label was added to natural seawater. This was done to assess the role of microbial degradation on the protective organic layer surrounding the frustule (Bidle \& Azam, 1999; Bidle et al., 2003). No autoclaving was done for the control incubation. Diatoms (mixture of T. pseudonana \& T. weisflogii) were added to the incubation seawater in $0.01 \mathrm{~mL}$ aliquots (resulting in $\sim 10^{5}$ cells per tube). For the time-series study the diatoms were kept in solution for 0.5 h, 1 h, 24 h, 168 h (1 week), 336 h (2 weeks), 672 h (4 weeks), and 1008 h (6 weeks), and incubated in a climate-controlled room at $15^{\circ} \mathrm{C}$. 
To test for temperature dependence, additional diatom incubations were performed at 4 ${ }^{\circ} \mathrm{C}$ (bottom-water conditions) and $15^{\circ} \mathrm{C}$ (surface-water conditions) for $336 \mathrm{~h}$. The same diatom mixture was used as in the previous experiments. As the frozen diatom samples had been diluted with MilliQ, these diatoms were suspended in liquid for an extended period. Possibly resulting in elevated dissolved silica (DSi) concentrations compared to the experiment described above.

The sponge spicules received a similar treatment as the diatoms. However, only spicules incubated for $24 \mathrm{~h}$ and $336 \mathrm{~h}$ (2 weeks), and a control sample incubated in natural seawater (NSW) for less than $1 \mathrm{~h}$, were analyzed. The quartz crystals and sand grain samples were only studied after $336 \mathrm{~h}$ (2 weeks) of incubation in the ${ }^{18} \mathrm{O}$ enriched and unlabeled seawater.

At the end of the incubation, the samples were centrifuged for $10 \mathrm{~min}(\mathrm{RCF}=15411 \mathrm{~g}, 20$ ${ }^{\circ} \mathrm{C}$ ) to settle the material. Supernatant $(1 \mathrm{~mL})$ was extracted and filtered through a sterile $0.2 \mu \mathrm{m}$ syringe-filter (VWR) for dissolved silicate determination. The remaining material was rinsed with autoclaved MilliQ and filtered onto a $0.2 \mu \mathrm{m}$ Nuclepore polycarbonate (PC) filter. The filter was then air-dried and stored in a Petri dish at $15^{\circ} \mathrm{C}$.

To check for potential changes in the $\delta^{18} \mathrm{O}$ of the water during incubation, the supernatant for two diatom samples (time-points $1 \mathrm{~h}$ and $336 \mathrm{~h}$ ) was analysed using OA-ICOS (LGR Off-Axis Integrated Cavity Output Spectroscopy). No significant change in $\delta^{18} \mathrm{O}_{\text {water }}$ was detected.

\subsubsection{NanoSIMS measurements - diatom frustules}

To prepare the samples for nanoSIMS analysis, dried PG-filters with the diatom detritus were coated with a $12 \mathrm{~nm}$ Au-layer using a sputter coater (JOEL JFC-2300HR high-resolution fine coater, JEOL FG-TM20 thickness controller). The frustules received no additional treatment prior or after this step. Target frustules were identified by imaging with a table-top Secondary Electron Microscope (JEOL JCM-6000PLUS NeoScope Benchtop SEM) operating at a 10-kV accelerating voltage.

Nanoscale secondary ion mass spectrometry was performed with the nanoSIMS 50L instrument (Cameca) operated at Utrecht University. Electron multiplier detectors were set using a standard (SPI Supplies, 02757-AB 59 Metals \& Minerals Standard) to enable the detection of secondary ions ${ }^{12} \mathrm{C}^{-},{ }^{16} \mathrm{O}^{-},{ }^{18} \mathrm{O}^{-},{ }^{12} \mathrm{C}^{14} \mathrm{~N}^{-},{ }^{28} \mathrm{Si}^{-}$and ${ }^{32} \mathrm{~S}^{-}$with the $\mathrm{Cs}^{+}$primary ion beam. First, samples were placed in the airlock to reach vacuum levels of $10^{-8} \mathrm{mbar}$ and heated to $\sim 50{ }^{\circ} \mathrm{C}$ for at least $24 \mathrm{~h}$ to remove any adsorbed water. After this, the samples were moved to the vessel chamber and kept in a vacuum $\left(10^{-9} \mathrm{mbar}\right)$ until analysis. For primary ion implantation, the lowenergy (LE) implantation technique was applied to preserve the target material. Initially, tuning and measurement were both performed on the same diatom frustule; however, this resulted in a significant loss of material, even with the primary ion current of $0.5 \mathrm{pA}$. Therefore, it was decided to perform tuning on a nearby diatom, thus preserving the entire target frustule for measurement. After the tuning, LE implantation was performed on the target frustule for $300 \mathrm{~s}$ after which the measurement was started. The imaged area varied among the measured specimens but ranged between $5 \times 5 \mu \mathrm{m}$ and $20 \times 20 \mu \mathrm{m}$. Images were acquired with a resolution of $128 \times 128$ pixels. To achieve the best spatial resolution, imaging was done with the primary ion current of $0.5 \mathrm{pA}$, the diaphragm and slit settings of D1-3, ES-3, AS-2, and a dwell time of $1 \mathrm{~ms} /$ pixel. Analysis generally continued until the frustules were largely sputtered away.

\subsubsection{NanoSIMS measurements - sponge spicules and crystalline silica}

Initially, the sponge spicules were prepared and measured in the same way as the diatom frustules. However, due to the lack of 'flat' areas, surface charging was a major issue. Furthermore, the solid 
structure did not allow the beam to completely sputter away the spicules with the currents used for the frustule analysis. Some broken spicules did provide cross-sections, but these surfaces were rarely well placed or sufficiently flat for the nanoSIMS analysis. Therefore, the sample preparation was adjusted.

Specifically, a circular portion $(0.5 \mathrm{~cm}$ diameter $)$ was cut from the filter, placed on a $1 \mathrm{~cm}$ conductive carbon tape and surrounded by an aluminum-ring. This ring was then filled with a resin (Araldite 2020), sonicated to remove air bubbles, and left to dry for at least $24 \mathrm{~h}$ at room temperature. After this, the carbon tape and filter were removed using P4000 silicon carbide paper. Subsequently, F1 diamond fluid was used to polish the surface. Finally, the polished surface was coated with a $12 \mathrm{~nm}$ thick Au-layer using a sputter coater. SEM analysis revealed that the inner parts of the spicules were well exposed and flat. Similar preparation steps were employed for the samples of the quartz crystals and sand grains.

NanoSIMS analysis of the spicules used the same instrument settings as the analysis of frustules. To prevent possible charging of the sample, the e-gun was used. Contrary to the frustule measurements, LE-implantation of the primary $\mathrm{Cs}^{+}$ions was not employed for the spicules as they appeared to be more robust and contained more material. Instead, pre-sputtering was performed with D1-1, 2 pA and C4Y:50 until secondary ion yields stabilized. Imaging was performed with the primary beam current of either $1 \mathrm{pA}$ or $2 \mathrm{pA}$, resolution of $128 \times 128$ pixels, dwell time of 1 ms/pixel, and over 300 planes. The analysis of the quartz crystals and sand grains proceeded in a similar manner.

\subsubsection{NanoSIMS data analysis}

NanoSIMS data were analyzed with an updated version of the Matlab-based freeware software Look@nanoSIMS (Polerecky et al., 2012). The general approach involved the analysis of ion count ratios in manually drawn regions of interest (ROIs), and their variation with depth (measurement plane) in the sample. Five critical observations guided the final data analysis strategy.

First, the ${ }^{18} \mathrm{O} /\left({ }^{16} \mathrm{O}+{ }^{18} \mathrm{O}\right)$ ion count ratio measured in diatom frustules increased significantly over the initial $\sim 50$ planes (Supplementary Fig. S4.1d). We attributed this to a combined effect of the sample preparation and measurement. Specifically, the frustules were enriched in ${ }^{18} \mathrm{O}$ as a result of their incubation with ${ }^{18} \mathrm{O}$-labeled seawater (see Results), but during sample preparation they were rinsed with unlabeled MilliQ. This resulted in a lower ${ }^{18} \mathrm{O}$ atom fraction in the very top layers of the frustule due to rapid ${ }^{16} \mathrm{O}_{-}{ }^{18} \mathrm{O}$ exchange. Because we first used the low-energy implantation technique to increase the secondary ion yields without sputtering away the frustule material, the measurement with the high-energy beam made it possible to detect this local decrease. Unfortunately, due to the lack of calibration between the measurement plane and depth within the sample, we were unable to estimate the range over which this surface-bound decrease occurred. Nevertheless, to avoid the influence of this surface effect, the initial $\sim 50$ planes were excluded from the analysis. These trends were not detected in the sponge spicules and crystalline silica materials because they were initially pre-sputtered with a high-energy beam.

Second, the frustule material was not sputtered uniformly during the analysis, leading to progressively increasing heterogeneity in the ion count images as the number of imaged planes increased (Fig. S4.1a-c). Most importantly, material from the underlying filter became detected in ROIs that corresponded to the frustule at the beginning of the measurement. Because the ions from the filter could strongly affect the final ion count ratios, only areas on the frustule which persisted throughout the measurement were used in the analysis. This was determined based on the ${ }^{28} \mathrm{Si}$ ion 
count image (Fig. S4.1e) combined with the depth profiles of the ${ }^{28} \mathrm{Si}$ ion counts and the ${ }^{18} \mathrm{O} /{ }^{16} \mathrm{O}$ ion count ratios in ROIs.

Third, the frustules often contained localized remnants of biomass, as revealed by the detected ${ }^{12} \mathrm{C}^{14} \mathrm{~N}$ ions. These organics had lower ${ }^{18} \mathrm{O}$ fractions than the frustule. To avoid the influence of this, areas with significant counts of the ${ }^{12} \mathrm{C}^{14} \mathrm{~N}$ ions were excluded from the analysis.

Fourth, for the embedded sponge spicules and quartz specimens, the imaged area always targeted both the sample and the resin to facilitate internal referencing of the ${ }^{18} \mathrm{O}$ atom fraction determination in the target material.

Finally, to rule out edge effects, edges of frustules were usually avoided during ROI definition. More details about this ROI approach can be found in Chapter 6 of this thesis.

\subsubsection{Raman spectroscopy}

Analysis was performed with the WITec Alpha 300R Raman spectrometer using the $532 \mathrm{~nm}$ line generated by a Nd-YAG laser. The instrument is equipped with an optical microscope and a 100x objective lens (numerical aperture of 0.8 ) allowing the measurement of lateral profiles across spicules and quartz crystals with a resolution of $\sim 1 \mu \mathrm{m}$ and depth profiles with a resolution of slightly larger than $1 \mu \mathrm{m}$. A grating of 600 grooves $/ \mathrm{mm}$ was used to obtain the entire spectral region of interest $\left(100-3700 \mathrm{~cm}^{-1}\right)$ in a single spectrum allowing direct comparison of bands in different regions. Signal to noise was improved by taking each spectrum for five seconds and averaging over 10 acquisitions. Background removal was performed using the WITec Project Plus software (version 4), and band fitting was conducted in Fityk (Wojdyr 2010).

For the spicules incubated in ${ }^{18} \mathrm{O}$-enriched water for two weeks, a $2 \mathrm{D}$ intensity map in the $\mathrm{x}, \mathrm{z}$ direction was created, as well as depth line scan analyses scanning the same $\mathrm{x}, \mathrm{y}$ point at increasing z. For the control spicule and quartz, only depth line scans were generated. A fossil frustule $(\sim 50 \mu \mathrm{m}$ diameter) with ${ }^{18} \mathrm{O}$-enrichment from Chapter 5 was also targeted for spectral analysis to determine water content. Fresh diatom samples were not studied because the depth resolution of the Raman spectroscopy $(\sim 1 \mu \mathrm{m})$ exceeds the thickness of the frustule valves, which is for T. pseudonana in the range 63-150 nm (Hildebrand et al., 2006), and this would not produce sufficient signal.

\subsection{Results}

\subsection{1 $\quad{ }^{18} \mathrm{O}$-enrichment in biogenic amorphous silica vs. crystalline silica}

After two weeks of incubation, there were no significant differences between the ${ }^{18} \mathrm{O}$-atom fractions measured in the crystalline silica samples (quartz and sand grains) incubated in ${ }^{18} \mathrm{O}$-enriched seawater and the control samples $(\mathrm{p}>0.05$; Table S4.3). This is in contrast to the biogenic silica samples, which showed significant enrichment in ${ }^{18} \mathrm{O}$ compared with the controls (Fig. 4.1). Of the biogenic silica samples, the ${ }^{18} \mathrm{O}$ enrichment was lowest in the sponge spicules $\left({ }^{18} \mathrm{O}\right.$ atom fractions around 2.8E-3) while it was quite substantial for the fresh diatom frustules $\left({ }^{18} \mathrm{O}\right.$ atom fractions around 3E-2). Fossil diatom frustules presented in Chapter 5 of this thesis also showed significant enrichment $\left({ }^{18} \mathrm{O}\right.$ atom fractions around 3.6E-2). No significant differences were observed for spicules that were remeasured after having spent three months in the vacuum $\left(10^{-9} \mathrm{mbar}\right)$ of the vessel chamber of the NanoSIMS instrument (data not shown). Repeated measurements of the very same frustules were not possible as the frustules were completely sputtered away during the nanoSIMS measurement. 


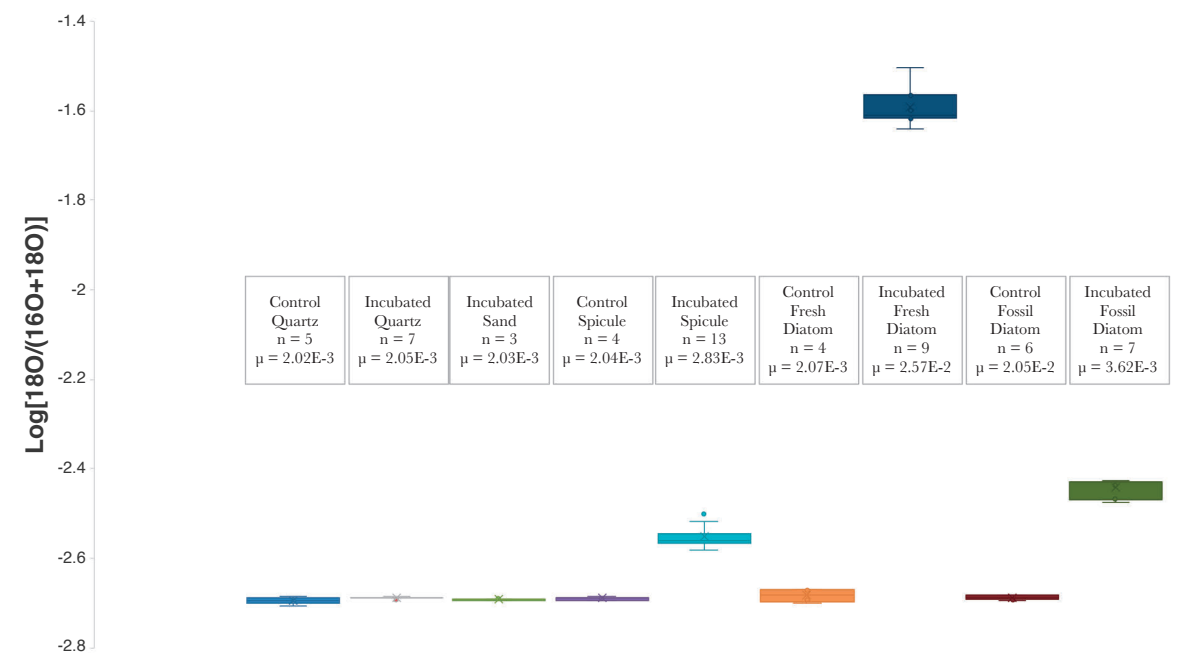

Figure 4.1: ${ }^{18} \mathrm{O}$ atom fractions in various forms of silica. Compared are control samples with samples incubated for 2 weeks in ${ }^{18} \mathrm{O}$-enriched seawater $\left({ }^{18} \mathrm{O}\right.$ atom fraction of $\left.\sim 0.09\right)$. Mean ${ }^{18} \mathrm{O}$ atom fractions and number of measured specimens are shown for each sample type. Note the logarithmic scale of the y-axis. The data was collected over several measuring sessions to confirm reproducibility of the measurements.

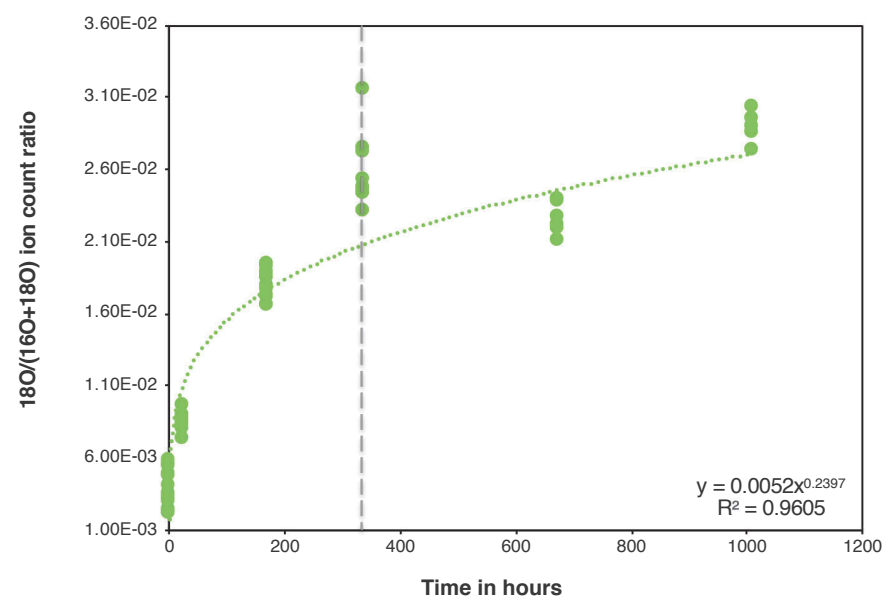

Figure 4.2: Time-evolution of the post-mortem ${ }^{18} \mathrm{O}$ atom fractions in diatom frustules. Dashed line indicates the 2-week mark. Trend is fitted with a power function. The dataset contains all the measured diatom samples including control frustules (data-points at $\mathrm{T}=0$ ), and frustules incubated in ${ }^{18} \mathrm{O}$-enriched seawater that was or was not autoclaved. Distinction between the latter two treatments is shown in Fig. S4.3 


\subsubsection{Rapid post-mortem ${ }^{18} \mathrm{O}$-enrichment in diatom frustules}

Diatom frustules incubated in ${ }^{18} \mathrm{O}$-enriched seawater at $15{ }^{\circ} \mathrm{C}$ showed rapidly increasing ${ }^{18} \mathrm{O}$ atom fractions, gradually decreasing in rate with time (Fig. 4.2). No significant differences were observed between the natural and autoclaved seawater samples at any time point measured (Fig. S4.3).

Images revealed that the ${ }^{18} \mathrm{O}$ atom fractions in the frustule valve $(8.3 \mathrm{E}-3)$ were significantly larger than in the girdle (7.1E-3; Fig. 4.3). The ${ }^{18} \mathrm{O}$ atom fraction was generally homogenous within the valve, both in the lateral and vertical dimensions (Fig. S4.1). Although the studied species have very similar morphology but slightly different frustule diameters (T. pseudonana: 4-7 $\mu \mathrm{m}$; T. weisflogii: 7-10 $\mu \mathrm{m})$, the differences in frustule diameter had no apparent effect on the measured ${ }^{18} \mathrm{O}$ atom fractions (Fig. S4.4).

Incubations in ${ }^{18} \mathrm{O}$-enriched seawater for 2 weeks revealed that frustules incubated at $4{ }^{\circ} \mathrm{C}$ had a significantly lower ${ }^{18} \mathrm{O}$ atom fraction than the frustules incubated at $15{ }^{\circ} \mathrm{C}$ (Fig. 4.4). However, both were significantly enriched compared to the control samples $(\sim 2 \mathrm{E}-3)$. Based on these two temperature-points, the activation energy could be estimated graphically using the Arrhenius equation, assuming first-order reaction kinetics. The resulting estimated activation energy was $\mathrm{E}_{\mathrm{a}}=$ $30.9 \pm 4.6 \mathrm{~kJ} \mathrm{~mol}^{-1} \mathrm{~K}^{-1}$.
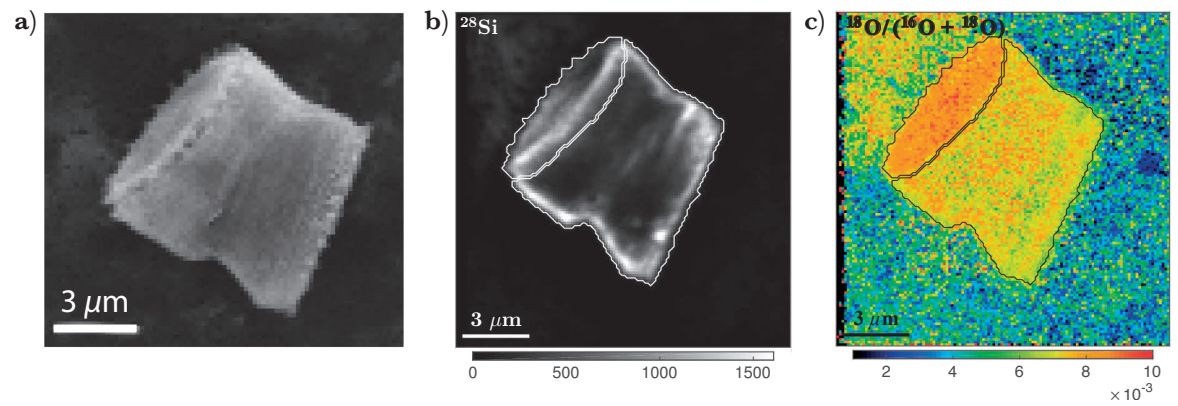

Figure 4.3: Distribution of the ${ }^{18} \mathrm{O}$ enrichment in a diatom frustule. Shown are images of a frustule incubated in ${ }^{18} \mathrm{O}$-enriched seawater for $24 \mathrm{~h}$. (a) SEM image of the side view of an intact frustule. (b) Accumulated 28Si ion count image. (c) Image of the ${ }^{18} \mathrm{O}$ atom fraction showing relatively greater enrichment in the valve. White and black lines in panels (b) and (c) mark the valve and the girdle. 

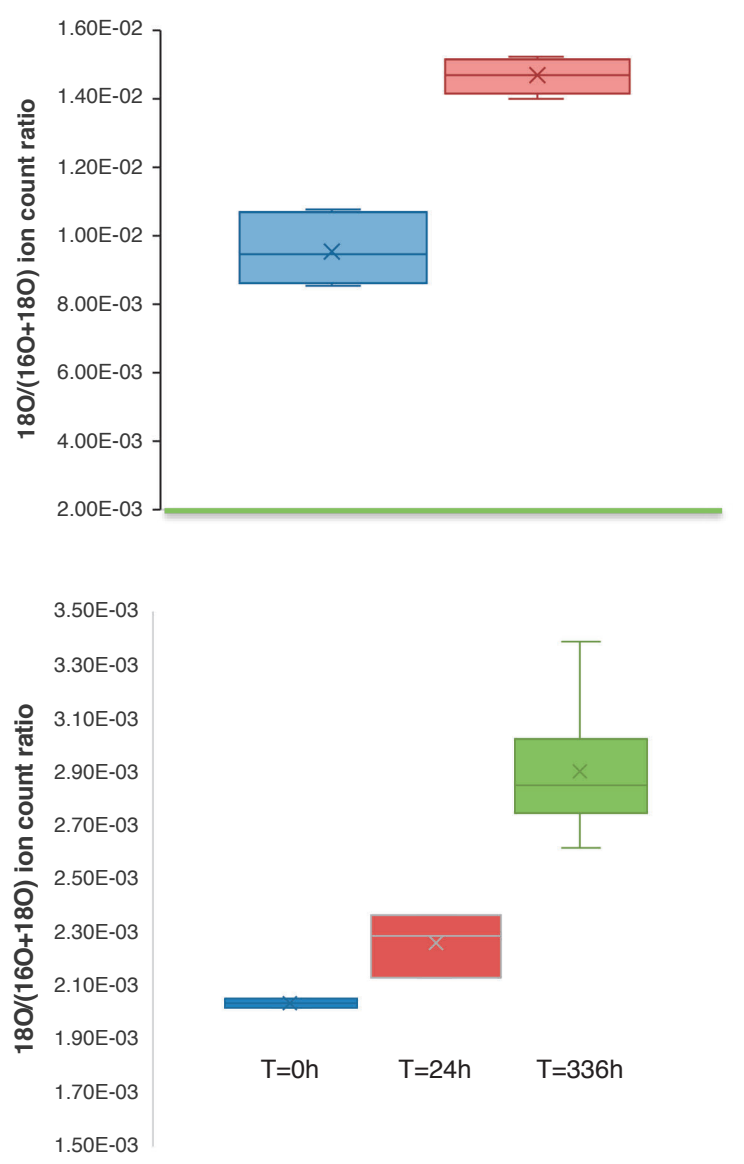

Figure 4.4: ${ }^{18} \mathrm{O}$ atom fractions in diatom frustules incubated for 2 weeks in ${ }^{18} \mathrm{O}$-enriched seawater at different temperatures. Green line represents the ${ }^{18} \mathrm{O}$ atom fraction in the control samples $(\sim 2 \mathrm{E}-3)$. Crosses $=$ mean values.

Figure 4.5: Time-evolution of ${ }^{18} \mathrm{O}$ atom fractions in sponge spicules incubated in ${ }^{18} \mathrm{O}$-enriched seawater. Mean values are marked by ' $\mathrm{x}$ '. The ${ }^{18} \mathrm{O}$ atom fraction of the resin in the background was significantly lower at $1.93 \mathrm{E}-3 \pm 0.04 \mathrm{E}-3$.

\subsection{3 $\quad{ }^{18} \mathrm{O}$-enrichment in sponge spicules}

Similar to diatom frustules, ${ }^{18} \mathrm{O}$ atom fractions in sponge spicules incubated in ${ }^{18} \mathrm{O}$-enriched seawater showed a clear increase over time (Fig. 4.5). The variability among specimens also increased with time (Fig. 4.5). However, after the same incubation time (2 weeks) the average ${ }^{18} \mathrm{O}$ atom fraction in the spicules $\left(2.84 \times 10^{-3}\right)$ was significantly lower than in the diatom frustules (2.57 $\times 10^{-2}$; Fig. 4.1).

The distribution of the ${ }^{18} \mathrm{O}$ atom fractions in the spicules was mostly homogeneous throughout the silica structure (Fig. 4.6). Occasionally, a locally increased ${ }^{18} \mathrm{O}$ enrichment was observed in areas of $0.5-1 \mu \mathrm{m}$ in size along the edges of the spicule (Fig. 4.6a), but these areas could not be identified based on the SEM and NanoSIMS data available. In several spicules, a depression (axial canal) running through the spicule was observed (Fig. 4.6d). This axial canal contained markedly higher amounts of $\mathrm{S}$ and was slightly more enriched in ${ }^{18} \mathrm{O}$ than the rest of the spicule silica (Fig. 4.6ef; Supplementary Fig. S4.5). Similar axial canals have previously been observed in hexactinellids (Sandford, 2003). 
a)

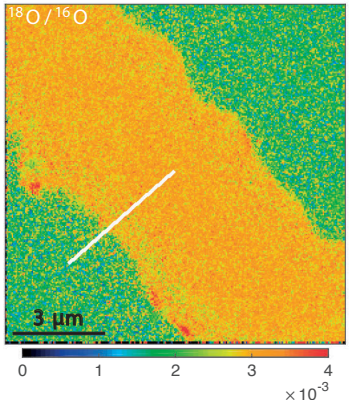

d)

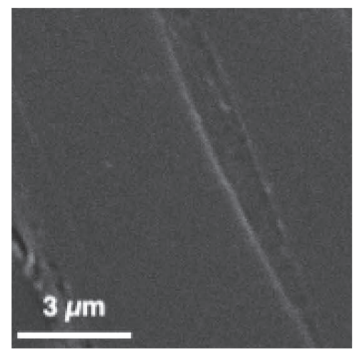

b)

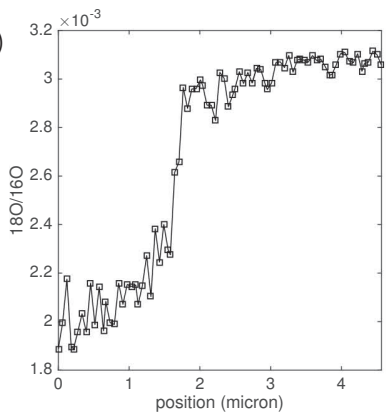

e)

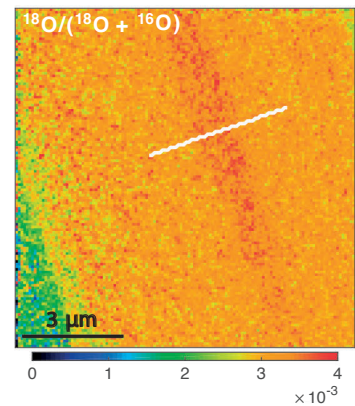

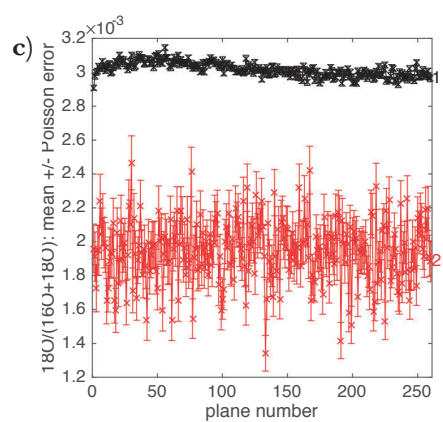

f)

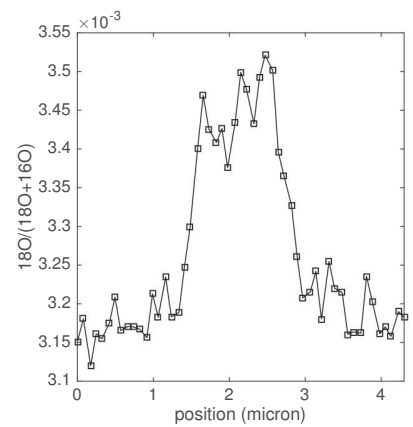

Figure 4.6: Distributions of ${ }^{18} \mathrm{O}$ atom fractions in sponge spicules incubated in ${ }^{18} \mathrm{O}$-enriched seawater for 2 weeks. (a) Image of the 18O/16O ion count ratio. (b) Lateral profile of the 18O/16O ratio along a line shown in panel (a). (c) Depth profile of the ${ }^{18} \mathrm{O}$ atom fractions in the spicule (black) and in the resin (red) (d) SEM image of another spicule showing a depression identified as the axial canal. (e) Image of the ${ }^{18} \mathrm{O}$ atom fraction in the spicule shown in panel (d). (f) Lateral profile of the ${ }^{18} \mathrm{O}$ atom fraction along a line shown in panel (e).

\subsubsection{Raman spectroscopy of amorphous and crystalline silica}

While nanoSIMS provides information on the distribution of oxygen isotopes, it does not provide information on the molecular structures in which the oxygen atoms are present. Raman spectroscopy, however, can distinguish these forms and may provide information about which of the potential O-bearing pools in the silica may have been contributing to the observed ${ }^{18} \mathrm{O}$-enrichment.

Table 4.1: Raman band assignments

\begin{tabular}{ll}
\hline Band position $\left(\mathrm{cm}^{-1}\right)$ & Band assignments \\
\hline a) $460-490$ & Si-O-Si stretching \\
b) $768-848$ & Inter tetrahedral Si-O-Si \\
c) $945-1010$ & Si-OH stretching \\
d) $1020-1134$ & Si-O-Si network \\
e) $3590-3610$ & $-\mathrm{OH}$ stretching within a solid Si structure \\
$3000-3700$ & Water $-\mathrm{OH}$ stretching region
\end{tabular}

Identification was based on (McMillan et al., 1982; Terpstra et al., 1990; Kammer et al., 2010; De Tommasi et al., 2018) 
a)

(a) (b) (c)(d)

(e)

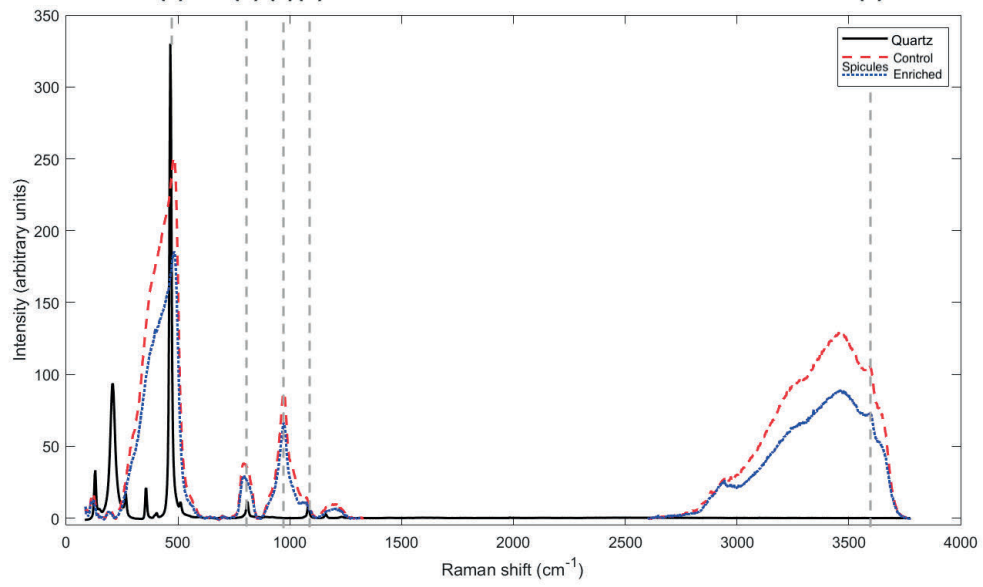

b)

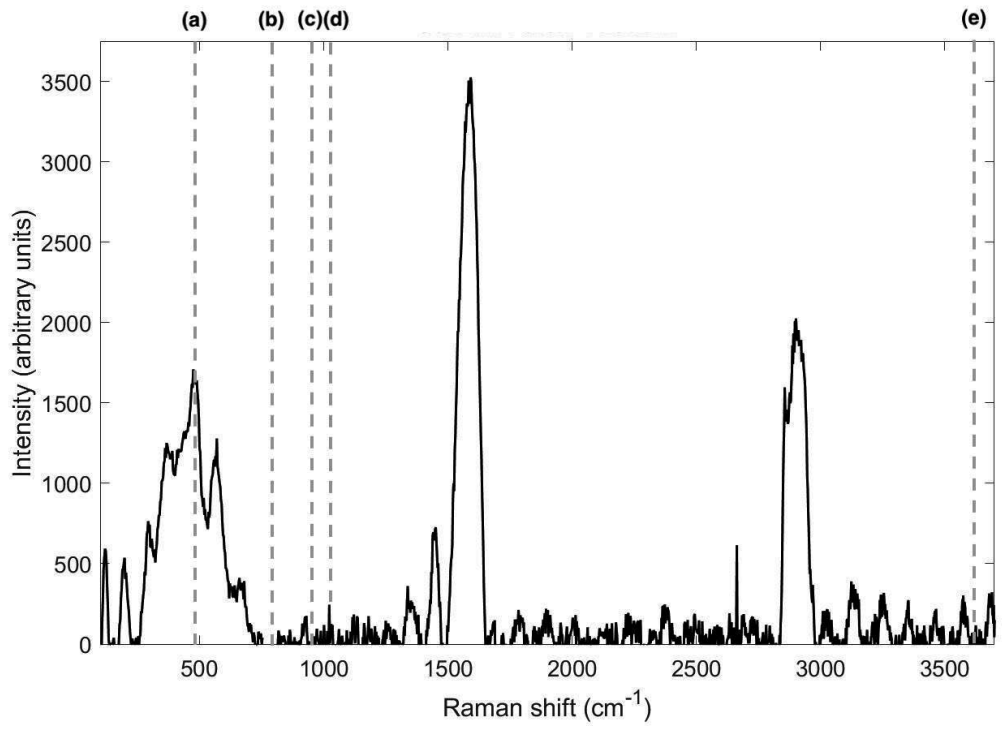

Figure 4.7: Raman spectra from sponge spicules, quartz and fossil diatom frustule. (a) Spicules and quartz. The spicule (blue line) and quartz (black line) were incubated in ${ }^{18} \mathrm{O}$-enriched seawater for 2 weeks. Red line corresponds to the control spicule. Identities of the target peaks are listed in Table 1. (b) Fossil diatom frustule. The frustule was incubated for two weeks in ${ }^{18} \mathrm{O}$-enriched seawater, and subsequently stored for five days in a vacuum $\left(10^{-9}\right.$ mbar) to remove any residual adsorbed water. Identities of the target peaks are listed in Table 1. Strong signals for aC-Hx $\left(1364-1438 \mathrm{~cm}^{-1}\right)$ and $v \mathrm{C}-\mathrm{Hx}\left(2826-3021 \mathrm{~cm}^{-1}\right)$ are visible in the spectrum but are not marked for this study. The ${ }^{18} \mathrm{O}$ atom fraction for this frustule was $3.57 \mathrm{E}-3$. 
A distinct feature in the Raman spectra of the sponge spicules (amorphous structure) is the peak around $975 \mathrm{~cm}^{-1}$, which is absent in the Raman spectra of quartz (crystalline structure) (Fig. 4.7a). This peak was attributed to the stretching of Si-OH groups (De Tommasi et al., 2018 and references therein). The presence of this group was further confirmed by the sharp band in the water region around $3610 \mathrm{~cm}^{-1}$, which is characteristic in vibrational spectroscopy for $\mathrm{O}-\mathrm{H}$ stretching within a solid Si structure rather than liquid water (Kammer et al. 2010). The [Si-O-Si $\left.i_{790 \mathrm{~cm}-1} / \mathrm{Si}_{-} \mathrm{OH}_{975 \mathrm{~cm}-1}\right]$ peak ratio did not vary between the control spicule and the spicule incubated in ${ }^{18} \mathrm{O}$-enriched water. The presence of $-\mathrm{OH}$ is one of the distinguishing characteristics of amorphous silica, as these groups cannot be present in crystalline silica due to the orderly and tightly packed atomic structure. The distribution of the -OH group within sponge spicules was also studied, but no spatial patterns were detected due to the low spatial resolution of the Raman measurements (Fig. S4.6).

Another distinctive feature in the Raman spectra of the sponge spicules was the broad water band around $3400 \mathrm{~cm}^{-1}$. Both the spicules and quartz used for measurements shown in Fig. 4.7a did not spend time in a vacuum. In order to check if water signals were the source of the ${ }^{18} \mathrm{O}$-enrichment observed in the nanoSIMS measurements, measurements were performed on samples that had spent five days in a vacuum $\left(10^{-8} \mathrm{mbar}\right)$. The measurements showed that the spectrum for spicule did not change (data not shown), meaning that the water signal was still present. However, we also analyzed a fossil diatom frustule that had been incubated in ${ }^{18} \mathrm{O}$-seawater, and here the water signals were absent (Fig. 4.7b), despite showing significant enrichment in ${ }^{18} \mathrm{O}$.

\subsubsection{Influence of heating on the ${ }^{18} \mathrm{O}$ atom fraction of sponge spicules}

Since the Raman measurements showed the presence of water in the silica structure after five days in a vacuum, the spicules previously measured by nanoSIMS were heated for an additional 7 hours in a vacuum of the NanoSIMS airlock $\left(10^{-8} \mathrm{mbar}\right)$ and remeasured. The exact temperature could not be determined but was estimated to be higher than $80{ }^{\circ} \mathrm{C}$ (M. Kienhuis, personal communication). This treatment increased the mean ${ }^{18} \mathrm{O}$ atom fraction from $2.83 \mathrm{E}-3$ to $2.93 \mathrm{E}-3$, but the increase was not statistically significant $(\mathrm{p}=0.391$; Supplementary table S4.5).

$\square$ Before heating $\square$ After heating

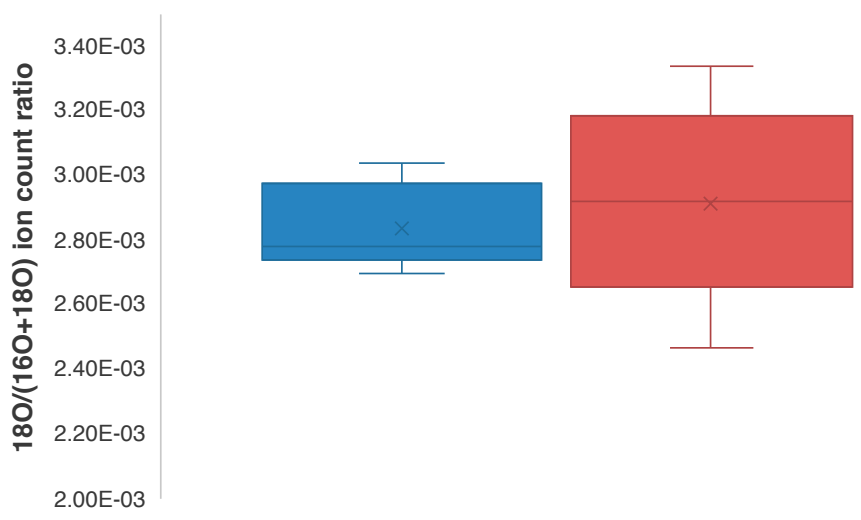

Figure 4.8: ${ }^{18} \mathrm{O}$ atom fractions of spicules before and after heating under vacuum. Data represents measurements from the same individual spicules $(\mathrm{N}=7)$. 


\subsection{Discussion}

This study revealed that biogenic silica undergoes a rapid resetting of the O-isotope signature when incubated in ${ }^{18} \mathrm{O}$-enriched water. Moreover, the exchange appears to take place not only at the surface hydroxyl groups but also in the rest of the silica matrix without an apparent alteration of the structure. This confirms concerns that have been raised regarding the role of diagenesis in influencing $\delta^{18} \mathrm{O}$ of fossil diatom frustules. While previous research has focused on diagenesis in the sediment, this study shows that oxygen isotope exchange can already take place rapidly in the water column. By comparing different forms of biogenic amorphous silica and crystalline silica, the significance of the isotope exchange in biogenic silica becomes clear.

\subsubsection{Post-mortem oxygen exchange in amorphous biogenic silica}

In the case of diatom frustules, their susceptibility to post-mortem oxygen exchange becomes evident from the rapid changes in ${ }^{18} \mathrm{O}$ atom fractions with time. After spending only 24 hours in the ${ }^{18} \mathrm{O}$-enriched seawater, we already observed significant changes, while after one week in ${ }^{18} \mathrm{O}$-enriched water, the frustules displayed ${ }^{18} \mathrm{O}$ atom fractions that are an order of magnitude higher compared to the natural abundance values of the control frustules. After six weeks, the rate of change slowed and the measured ${ }^{18} \mathrm{O}$ atom fraction of the frustules stabilized around 2.9E-2, which was about a third of the water signal. This study confirms an earlier study which also shows similar alterations on the timescale of days to weeks (Tyler et al., 2017). Considering that diatoms may spend around 2-4 weeks in the water column before reaching the seafloor, a considerable part of the original ${ }^{18} \mathrm{O}$ oxygen signal will have been overprinted before the frustule reaches the sediments.

In this study, the rate of change decreases over time, likely in part due to the decreasing difference between the ${ }^{18} \mathrm{O}$ atom fraction of the water and the frustule. Over longer timescales a decrease in reactivity is also expected due to other processes. In sediment samples decreasing reactivity is often attributed to the diminishing number of silanol groups by silica maturation (Dodd et al., 2012; Schmidt et al., 2001; Moschen et al., 2006; Lewin, 1961; Brandriss et al., 1998 among others). Despite the decreasing reactivity, the impact over geological timescales can be substantial. This decreasing but persistent reactivity was also acknowledged by Dodd et al. (2017), who mentioned that "the dehydroxylation process has the potential to continue to alter $\delta^{18} \mathrm{O}$ values for $\sim 10^{4}-10^{6}$ years."

A potential inhibitor of post-mortem oxygen transfer in the water column is the presence of a protective organic membrane surrounding the frustule (Moschen et al., 2006). Indeed, the presence of the organic membrane has been shown to strongly reduce silicon dissolution rates, confirming its protective nature when not strongly altered by the presence of bacteria (Bidle and Azam, 1999, 2001). Observations of varying $\delta^{18} \mathrm{O}$ in settling diatom detritus in a lacustrine environment are instead attributed to resuspended sedimentary frustules affecting the deep trap rather than rapid frustule diagenesis in the water column (Moschen et al., 2006). In an attempt to assess the role of the protective organic membrane in inhibiting secondary oxygen exchange in this study, experiments were performed with both natural seawater (biotic) as well as autoclaved seawater (abiotic). By autoclaving the water, the bacterial population should be minimized, preventing attacks on the organic membrane, leaving it intact. The ensuing data showed no significant differences in dissolution (Fig. S4.2) or ${ }^{18} \mathrm{O}$-exchange (Fig. S4.3) between the two environments, suggesting that the NSW experiment likely also had very little bacterial activity. The observed ${ }^{18} \mathrm{O}$-enrichment in 


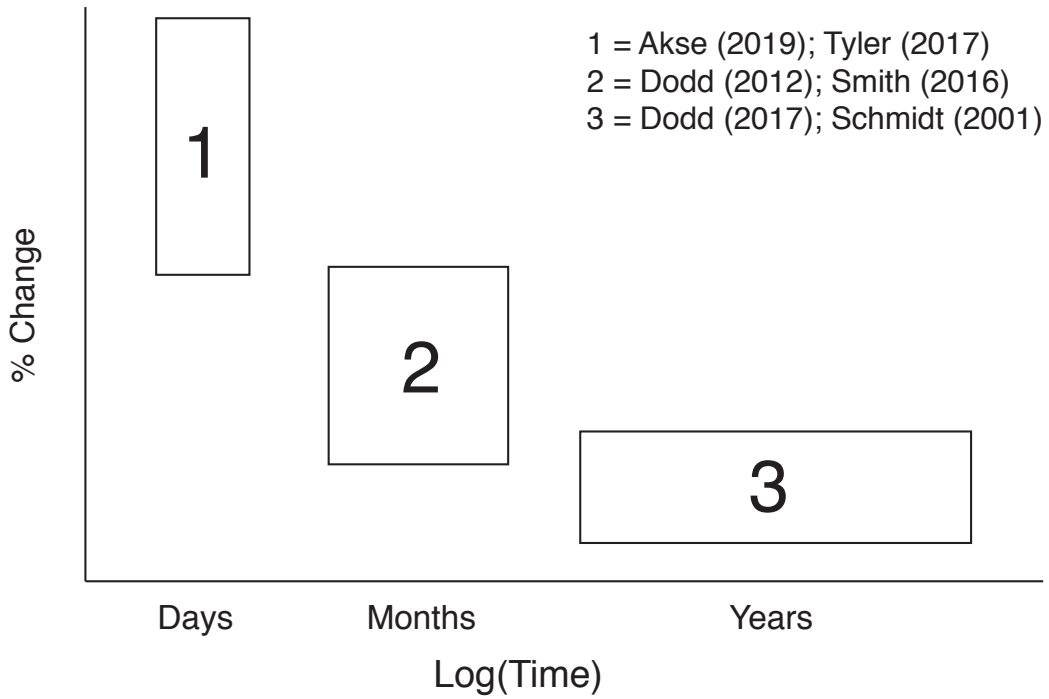

Figure 4.9: Schematic diagram putting this study in a context of different studies looking at diagenetic overprinting of frustule $\delta^{18} \mathrm{O}$.

the frustules incubated in autoclaved water suggests that post-mortem oxygen exchange is possible despite the potential presence of a protective membrane.

Instead of dissolution, the hydrous $\mathrm{Si}-\mathrm{OH}$ layer is seen as the main contributor to the differences observed in $\delta^{18} \mathrm{O}_{\text {frustule }}$ of surface diatoms and those found in the sediment (e.g. Brandriss et al., 1998; Moschen et al., 2005; Dodd \& Sharp 2010). Though the diatom data in this study clearly confirms the post-mortem interaction of diatom frustules with their surrounding waters, the porous and relatively thin morphology of the frustules makes it challenging to determine if only surface silanol groups were affected or if the tetrahedrally bonded silica was similarly altered. Therefore, the experiment was repeated with siliceous sponge spicules, which have a comparable amorphous silica matrix but a thicker and larger physical structure, providing more material for nanoSIMS measurements.

Similar to the diatom samples, spicules incubated in ${ }^{18} \mathrm{O}$-enriched seawater displayed increased ${ }^{18} \mathrm{O}$ atom fractions. The 2-week incubated spicules showed an increase in ${ }^{18} \mathrm{O}$ atom fraction from $\sim 2 \mathrm{E}-3$ to $\sim 3 \mathrm{E}-3$ (Fig. 4.5). Some spicules were remeasured after spending three months in a vacuum at $20{ }^{\circ} \mathrm{C}$ while others were remeasured after spending seven hours in a vacuum under increased temperatures $\left(>80^{\circ} \mathrm{C}\right)$. These spicules showed reproducible values, suggesting that adsorbed water was sufficiently removed for all measurements and did not play a role in the observed ${ }^{18} \mathrm{O}$-enrichment. Instead, it appears that entire spicule can become enriched with ${ }^{18} \mathrm{O}$ in a matter of several days. Compared to diatom frustules, the rate of exchange is lower in spicules, probably due to the reduced surface area. Unfortunately, the exact rate cannot be obtained from this experiment. To determine this, unknowns such as surface area and silanol abundance need to be constrained, which is outside the scope of this manuscript. The increasing variability of values as time progresses further demonstrates the need to constrain these uncertainties for future work. 


\subsubsection{Oxygen exchange in crystalline silica}

By measuring additional forms of silica, it became clear that, unlike the biogenic amorphous silica of sponge spicules and diatom frustules, crystalline silica was not affected by oxygen overprinting. Both quartz crystals and sand grains did not show changes in their ${ }^{18} \mathrm{O}$ atom fractions after spending time in the ${ }^{18} \mathrm{O}$-enriched water. This is an important observation for multiple reasons. First, these results confirm that the elevated ${ }^{18} \mathrm{O}$ atom fractions in the embedded and polished spicules cannot be attributed to sample preparation, as the same methodology was used for the crystalline silica. Second, this means that the secondary exchange of oxygen on the timescale of weeks is a phenomenon reserved for biogenic amorphous silica.

\subsubsection{Distribution of ${ }^{18} \mathrm{O}$ in biogenic amorphous silica}

For the $\delta^{18} \mathrm{O}$ proxy in biogenic silica, it is vital that we further understand the effect of diagenetic processes. Therefore, the chemistry of the individual tests was studied at a sub-micron level. In this way, it was possible to determine whether the oxygen exchange only took place at local hotspots, as a surface deposit or throughout the entire silica structure.

\section{Diatom frustules}

Our analyses showed that the ${ }^{18} \mathrm{O}$ atom fraction was affected uniformly throughout the silica matrix of the diatom valve. This was clear from both the homogenous lateral distribution in the ${ }^{18} \mathrm{O}$ atom fraction image as well as from the stable ${ }^{18} \mathrm{O} /{ }^{16} \mathrm{O}$ ratios with depth for the frustule silica (Fig. S4.1). In the depth profiles, the high ${ }^{18} \mathrm{O}$ values persisted for as long as the frustule silica was detected. The detected ${ }^{18} \mathrm{O}$ atom fraction started to decrease not because the frustule material was less enriched in ${ }^{18} \mathrm{O}$, but because of increasing interference from the underlying substrate as the thin frustule was sputtered away (Fig. S4.1). Laterally, the diatom girdles were less enriched in ${ }^{18} \mathrm{O}$ than the valves (Fig. 4.3), but as valves are commonly represented in the sediment archive, these were the focus of this study. Our NanoSIMS data from the diatom frustules could not conclusively distinguish between the enrichment of the hydroxyl groups and a possible enrichment of the tetrahedrally bonded oxygen. What they do tell us, however, is that the oxygen in the ambient water molecules can similarly affect both the external silanols as well as the internal silanols (Loucaides et al., 2010).

This is of importance as studies have shown that internal silanol groups may be more abundant than external silanols, as well as more difficult to remove with the current dehydroxylation techniques (e.g., Dodd et al. 2017). Furthermore, as studies have suggested that the condensation of Si-OH groups might be responsible for $\delta^{18} \mathrm{O}_{\text {diatom }}$ changes during maturation (e.g., Dodd et al., 2017; Schmidt et al., 2001), infiltrating secondary oxygen could become part of the silica matrix throughout the frustule. So even though the presence of an organic membrane encompassing the frustule prevents rapid dissolution (Bidle \& Azam, 1999), a small amount of infiltrating water can start this reaction. This suggests that current dehydroxylation techniques are not sufficient to remove the entire secondary oxygen signal.

\section{Sponge spicules}

Generally, the measured spicules showed relatively homogenous ${ }^{18} \mathrm{O}$ atom fractions throughout. Variations only occurred in the axial canal and as small hotspots at the edge between the spicule and the resin. The source of the hotspots could not be determined, but these were not consistently present throughout the measurements. The presence of enriched surface silanol groups seemed to be confirmed by the elevated ${ }^{18} \mathrm{O}$ atom fractions observed in the axial canals. The labeled water was likely able to invade the axial canal and exchange oxygen with the - $\mathrm{OH}$ groups. Similar to 


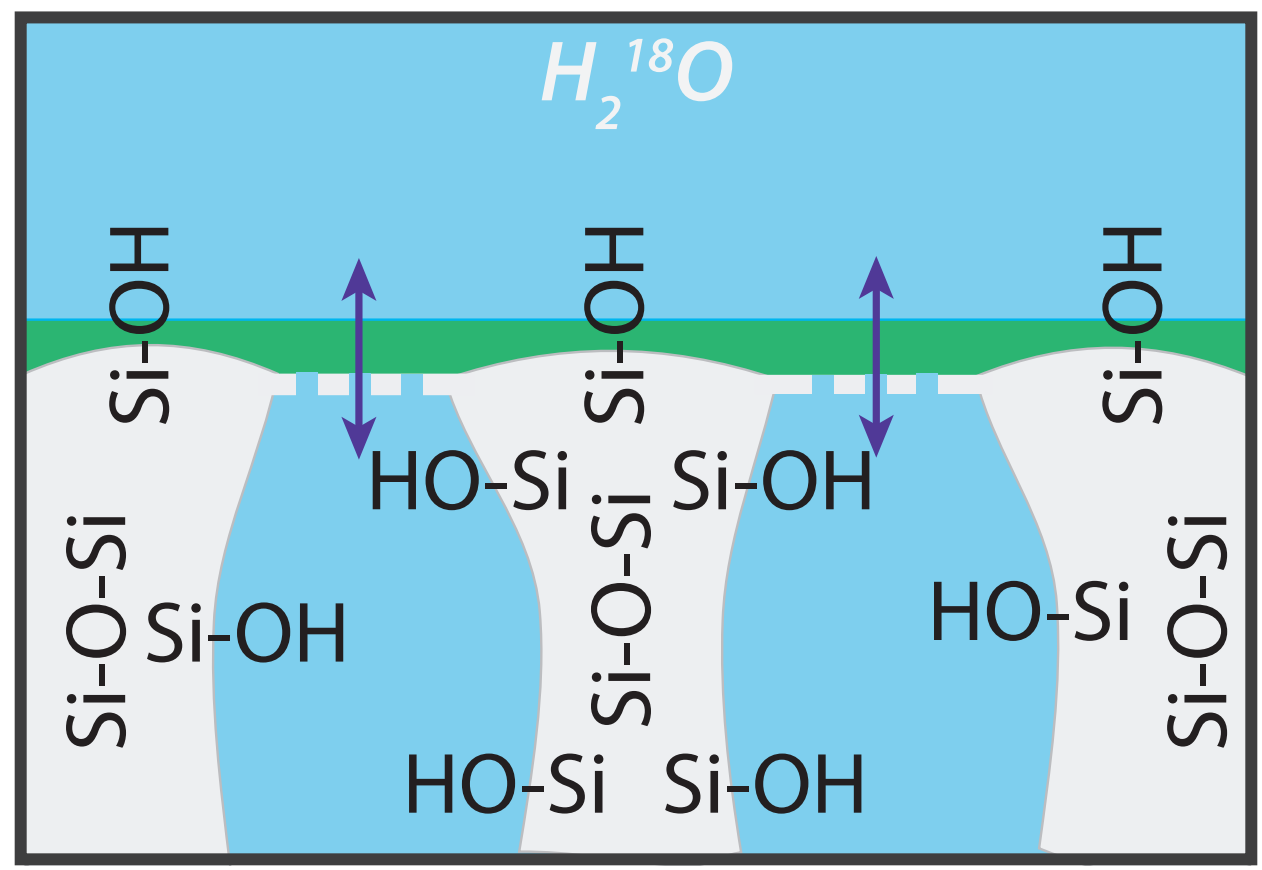

Figure 4.10: Schematic diagram of oxygen exchange between the diatom frustule and ambient water. Despite the presence of an organic membrane surrounding the frustule, ${ }^{18} \mathrm{O}$ enriched water infiltrates into the frustule pores leading to exchange of oxygen with internal silanols.

the ${ }^{18} \mathrm{O}$ signal, the $32 \mathrm{~S}$ signal was also elevated in the axial canal (See Fig. 4.6 and Fig. S4.5). A similar observation was previously made in calcareous sponge spicules (Kopp et al., 2011) where the detected $\mathrm{S}$ was attributed to the presence of sulphated polysaccharides embedded in the organic matrix of the biomineral. It is possible that the presence of proteins enhanced the uptake of secondary oxygen. However, it is more likely that the observed ${ }^{18} \mathrm{O}$ enrichment was a result of the presence of surface silanols. These observations imply two active processes: the swift oxygen exchange and condensation of labile hydroxyl groups followed by a slower diffusion of oxygen into the rest of the opal matrix.

\subsubsection{Raman measurements on amorphous and crystalline silica}

A significant result from the Raman measurements was the reproducibility of the data among the different spicules, meaning that the observed isotope exchange was not related to a physical phase change such as recrystallisation. Moreover, according to the Raman measurements, the main distinguishing feature of the spicule relative to the quartz was the presence of Si-OH, which suggests that the presence of silanol groups in biogenic silica is indeed an enabler of oxygen isotope exchange. Moreover, the more open nature of the amorphous silica structure enables more facile diffusion of water and consequently oxygen. Thus, the availability of reactive silanol groups and the open amorphous structure of biogenic silica allow the diagenetic overprinting of oxygen signal in the entire silica matrix. 


\subsubsection{The potential sources of oxygen signal}

The nanoSIMS results show a homogenous distribution of ${ }^{18} \mathrm{O}$ through the amorphous silica structures of the diatom frustules and sponge spicules. However, the nanoSIMS does not provide information on the structural site in which the oxygen atom is located. Within the silica, there are four potential O-bearing pools: (1) water adsorbed to the silica, (2) water trapped within the silica architecture, (3) silanol (Si-OH) units (Knauth and Epstein, 1982) and (4) siloxane (Si-O-Si) within the silica structure.

The first pool, loosely adsorbed water, is removed before measurement by initial drying of the samples in air as well as by degassing in the vacuum chambers of the nanoSIMS instrument. Therefore, this pool should not contribute to the measured signal. Determining the relative contribution of the other three pools (crystalline water, siloxane and silanol) is more challenging as the Raman measurements of the spicules show that all three are present within the structure (Fig. 4.7) even after exposure to the nanoSIMS vacuum for five days. It is likely that water entered the structure as the activation energy of ${ }^{18} \mathrm{O}$ exchange in diatoms was estimated $\left(\sim 31 \mathrm{~kJ} \mathrm{~mol}^{-1} \mathrm{~K}^{-1}\right)$ to be higher than the activation energy of self-diffusion of free liquid water $\left(\sim 19 \mathrm{~kJ} \mathrm{~mol}^{-1} \mathrm{~K}^{-1}\right.$; Mills, 1973; Fripiat et al., 1984). Water might be present in the ultramicropores (size $<1 \mathrm{~nm}$ ) and in the $\mathrm{OH}$ groups that are located at the walls of these pores, classified as internal silanols (Zhuravlev, 2000; Sato et al., 2011). Together with external silanol groups, these pools account for $7-12 \%$ of the total oxygen presence (Knauth et al., 1973). The remaining $\sim 90 \%$ can be attributed to oxygen in siloxane groups ( $\mathrm{Si}-\mathrm{O}-\mathrm{Si})$.

Considering the magnitude of the ${ }^{18} \mathrm{O}$ atom fraction observed in the diatom frustules (reaching $\sim 30 \%$ of the ${ }^{18} \mathrm{O}$ atom fraction of the incubation seawater), and the small contribution of silanol groups to the total $\mathrm{O}$ pool, our results suggest that oxygen in the siloxane groups of the frustules must also be altered due to the secondary oxygen exchange with the surrounding water.

In the spicules the presence of internal oxygen-bearing water in the silica matrix (even after having spent several days in the vacuum) is undeniable and this could contribute to the ${ }^{18} \mathrm{O}$-enrichment. However, it is unlikely that this pool contributed significantly to the ${ }^{18} \mathrm{O}$-enrichment observed by nanoSIMS. Firstly, fossil diatom frustules incubated in ${ }^{18} \mathrm{O}$-enriched water for 2 weeks showed higher ${ }^{18} \mathrm{O}$ enrichment than spicules, but Raman spectroscopy did not detect any water in these samples (Fig. 4.7b).

Secondly, ${ }^{18} \mathrm{O}$ atom fraction measured by nanoSIMS increased with the exposure time of the samples to the ${ }^{18} \mathrm{O}$-enriched water (Fig. $4.2 \& 4.5$ ). The lack of a diffusion profile across the spicule samples indicates that diffusion into the pores was efficient on the timescale of the experiments. In fact, in the depth profiles of fresh frustules, an indication of a very rapid diffusion found that is likely due to a very brief (minutes) exposure to MilliQ. This hints at the efficiency to which the water diffuses into the structure. Therefore, if water in the pores had a significant contribution in the nanoSIMS measurements, the ${ }^{18} \mathrm{O}$ atom fractions would be stable, rather than increasing with time.

Lastly, volatilization of water through heating to $\sim 100{ }^{\circ} \mathrm{C}$ has been found previously to occur without isotopic exchange with silica-bound oxygen (Labeyrie, 1974). Therefore, it is unlikely that the water signature can give rise to the ${ }^{18} \mathrm{O} /{ }^{16} \mathrm{O}$ signatures through exchange during sample pretreatment for nanoSIMS analysis. Indeed, even additional heating of the spicules under vacuum in the nanoSIMS airlock did not result in significantly lower ${ }^{18} \mathrm{O}$ atom fractions (Fig. 4.8).

Water within the diatoms appears to be removed easier than from the spicules, most likely due to the thinner and more open architecture of the frustules. If water was indeed removed from the frustules, only the hydroxyl and siloxane groups would remain. Moreover, the presence of 
structurally bound water may actually aid the diffusion processes of oxygen into the silica structure by providing an internal pool directly influenced by the external water signature with which internal silanols can exchange. If condensation of the internal silanols occurs due to maturation (Dodd et al., 2017) or sample preparation (Tyler et al., 2017), then the ${ }^{18} \mathrm{O}$ signal of the intrinsic water would become part of the silica structure.

\subsection{Conclusion}

Our data show that the oxygen signal in biogenic amorphous silica can become rapidly overprinted post-mortem, supporting recent concerns in the literature about early diagenetic effects on $\delta^{18} \mathrm{O}_{\text {diatom. }}$. While these concerns have focused predominantly on sediment diagenesis, we show that post-mortem diagenesis can already start playing a major role in the water column. This hypothesis is based on the observation that two forms of biogenic silica (diatom frustules and sponge spicules), when placed in ${ }^{18} \mathrm{O}$-enriched seawater, become rapidly enriched in ${ }^{18} \mathrm{O}$. NanoSIMS analysis of individual frustules showed that this enrichment occurred throughout the structure. This means that, at the very least, both external and internal silanol groups are similarly affected. Additional mapping of sponge spicules revealed that, despite the more solid structure of the spicules, the entire silica matrix was enriched in ${ }^{18} \mathrm{O}$, albeit to a lesser degree than the porous diatom frustules. Moreover, a similar experiment with crystalline silica (which lack hydroxyl groups) did not show enrichment in the ${ }^{18} \mathrm{O}$ atom fraction. These experiments confirm that the presence of surface silanol groups can lead to a rapid exchange of oxygen between biogenic silica and the ambient water. However, as the entire silica matrix appears to be altered, these experiments suggest that siloxane groups are also affected by secondary oxygen overprinting. This implies that despite the current dehydroxylation methods (see Chapligin et al., 2011 for a comparative study), the final measured $\delta^{18} \mathrm{O}_{\text {diatom }}$ from the frustule will most likely represent a mixed signal from the original growing stage, water-column settling, sediment-water interface, and sediment pore waters. As reactivity has been shown to decrease with time (Fig. 4.9), the oxygen signal may be dominated by early conditions, but continued reactivity on geological timescales cannot be ruled out. Therefore, records may still show large-scale trends, but detailed interferences about environmental conditions should be avoided. 


\section{Supplementary material}

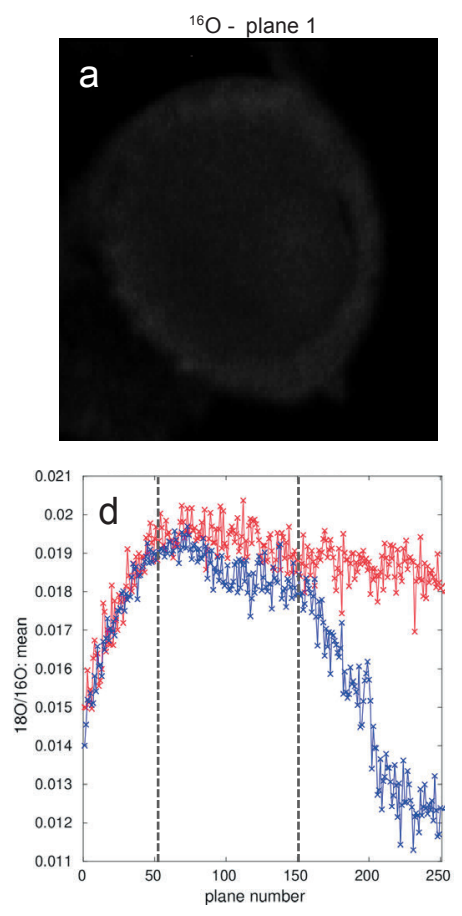

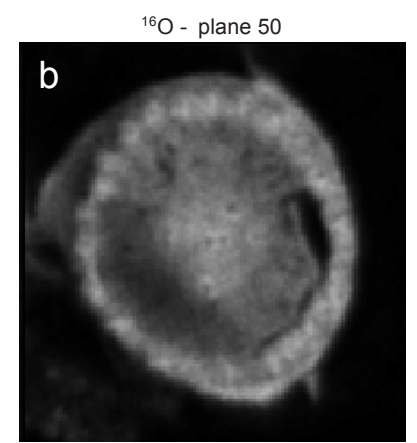

${ }^{16} \mathrm{O}{ }^{28} \mathrm{Si}{ }^{12} \mathrm{C}$ planes $50-150$

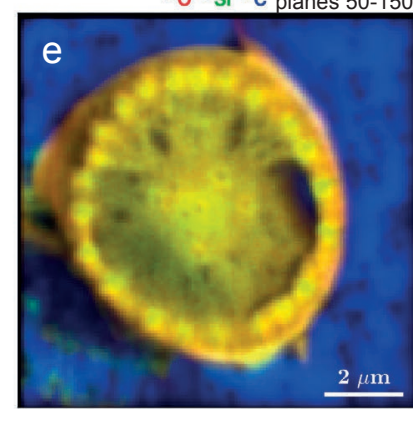

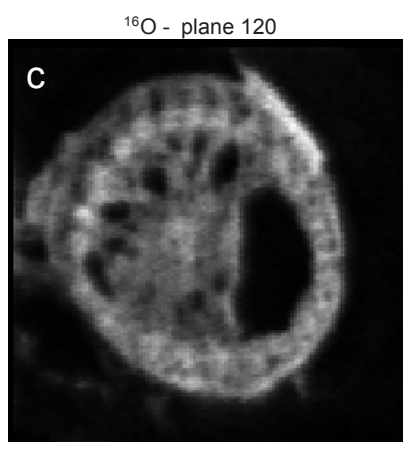

${ }^{18} \mathrm{O} /{ }^{16} \mathrm{O}$ - planes $50-150$

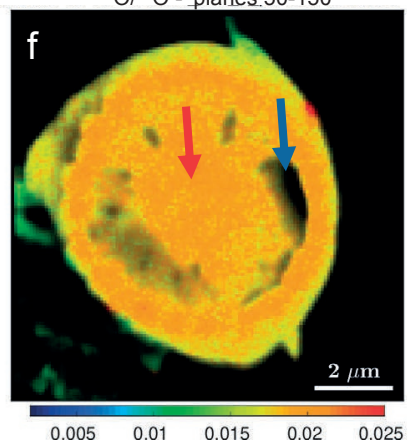

Figure S4.1: NanoSIMS imaging of $\mathbf{1 8 O} / \mathbf{1 6 O}$ in a diatom frustule. $(\mathrm{a}-\mathrm{c})$ Variation in the ${ }^{16} \mathrm{O}$ ion counts with the measurement plane. (a) Initially, ion counts are low due to insufficient implantation of the primary $\mathrm{Cs}^{+}$ions into the frustule matrix by the low-energy implantation procedure performed before the measurement. (b) As more highenergy $\mathrm{Cs}^{+}$ions are implanted into the matrix during the measurement, the secondary ion yield increases leading to higher ion counts. (c) However, sputtering of the frustule material is uneven, leading to increasing heterogeneity of the ion count image. Dark pixels correspond to areas where the frustule was completely sputtered away. $(\mathbf{d}){ }^{18} \mathrm{O} /{ }^{16} \mathrm{O}$ ratio as a function of the measurement plane (i.e., depth), shown for two areas on the frustule (red and blue data points correspond to areas pointed to by the red and blue arrow in panel $\mathrm{f}$ ). Following an initial increase (planes 0-50), the ${ }^{18} \mathrm{O} /{ }^{16} \mathrm{O}$ ratio stabilizes (planes $50-150$ ), but then starts to decrease towards the value corresponding to the underlying substrate (carbon-tape) as the frustule matrix becomes sputtered away (planes 150-250). The final mean was calculated using planes where the ${ }^{18} \mathrm{O} /{ }^{16} \mathrm{O}$ ratio was stable (see interval bound by the dotted lines). (e) Composite RGB image showing the distribution of the ${ }^{16} \mathrm{O}$ (red), ${ }^{28} \mathrm{Si}$ (green) and ${ }^{12} \mathrm{C}$ (blue) ion counts accumulated over planes 50-150. The frustule lying on top of the carbon-tape substrate (blue) is clearly visible. $(\mathbf{f}){ }^{18} \mathrm{O} /{ }^{16} \mathrm{O}$ image obtained from the ion count images accumulated over planes 50-150. Only pixels with sufficiently high ${ }^{16} \mathrm{O}$ ion counts are displayed. Pixels where the ${ }^{12} \mathrm{C}$ ion counts were significant (areas corresponding to the carbon-tape) are shown as dark. 


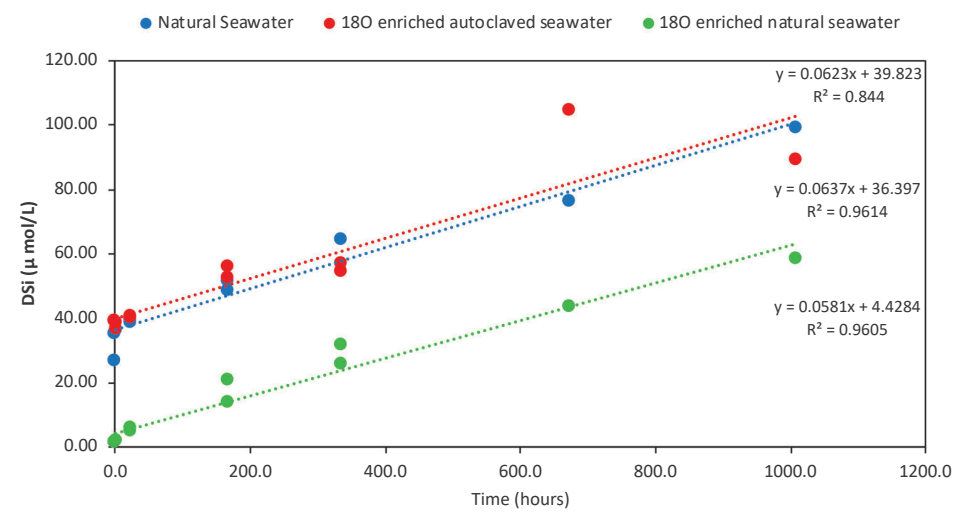

Figure S4.2: DSi changes over time during incubation experiments in ${ }^{18} \mathrm{O}$-enriched seawater. Shown are the influences of three different treatments on the DSi of the water. Diatom frustules were incubated either in natural seawater, ${ }^{18} \mathrm{O}$-enriched seawater or autoclaved ${ }^{18} \mathrm{O}$-enriched seawater. Each datapoint represents the DSi concentration in the supernatant of an individual experiment vial. The DSi concentrations displayed a linearly increasing trend with slopes $(0.062 \pm 0.010)$ that did not significantly differ among the three treatments $(\mathrm{p}=0.794$; Supplementary table S8). This indicates that diatom frustule dissolution rates were similar in all treatments. Starting conditions of the natural seawater samples were lower than those containing the $\mathrm{H}_{2}{ }^{18} \mathrm{O}$ label due to the glass vial in which the label was stored by the manufacturer before it was used for this study.

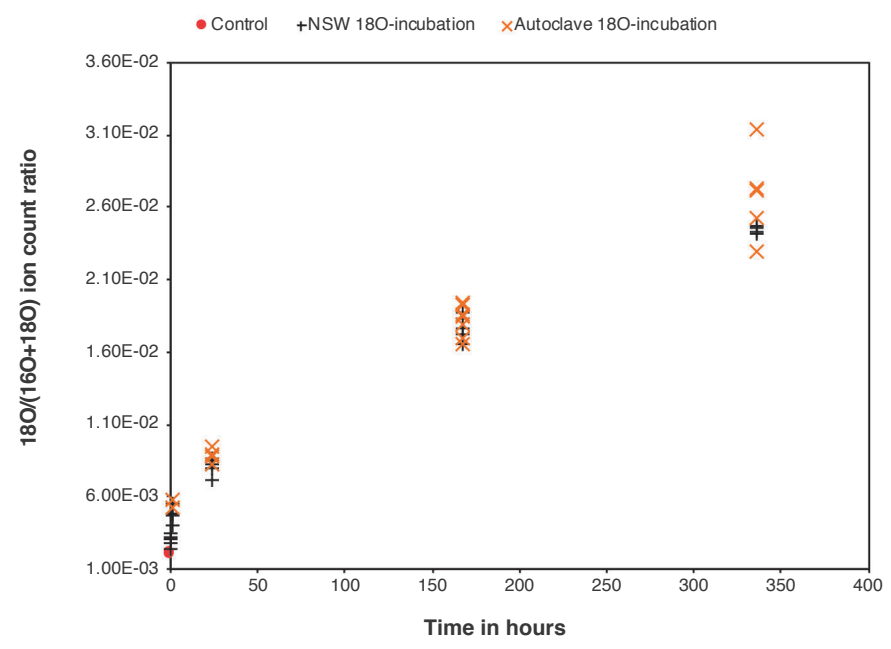

Figure S4.3: Post-mortem ${ }^{\mathbf{1 8}} \mathrm{O}$ enrichment under different experimental conditions. Shown are the ${ }^{18} \mathrm{O}$ atom fractions for the three different incubation treatments: Natural seawater, ${ }^{18} \mathrm{O}$-enriched natural seawater and ${ }^{18} \mathrm{O}$-enriched autoclaved seawater. Each datapoint represents an individual frustule. No differences are observed between the labelled incubations. The $12 \mathrm{C} 14 \mathrm{~N} / 28 \mathrm{Si}$ ratio did also not show differences between the treatments (data not shown) 


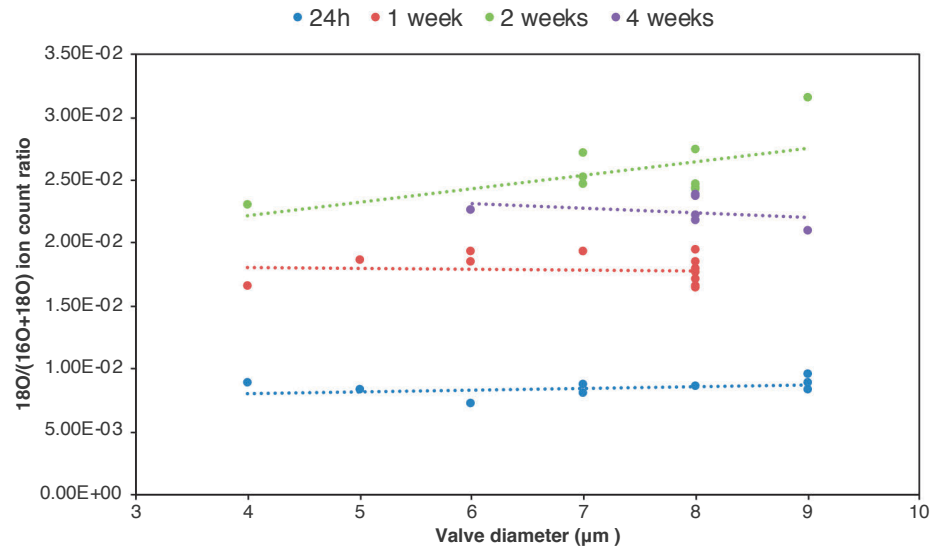

Figure S4.4: Influence of the frustule valve diameter on the 180 atom fraction. Shown are the $1^{8} \mathrm{O}$ atom ratios at different timepoints. Each datapoint represents an individual frustule. Smaller valves are often associated with T. pseudonana while the larger valves are associated with T. weisflogii. A statistical test could not be performed with confidence on this data due to the uneven distribution of the data over the different valve diameters, with some treatments showing a complete absence of data points for certain vale diameters.
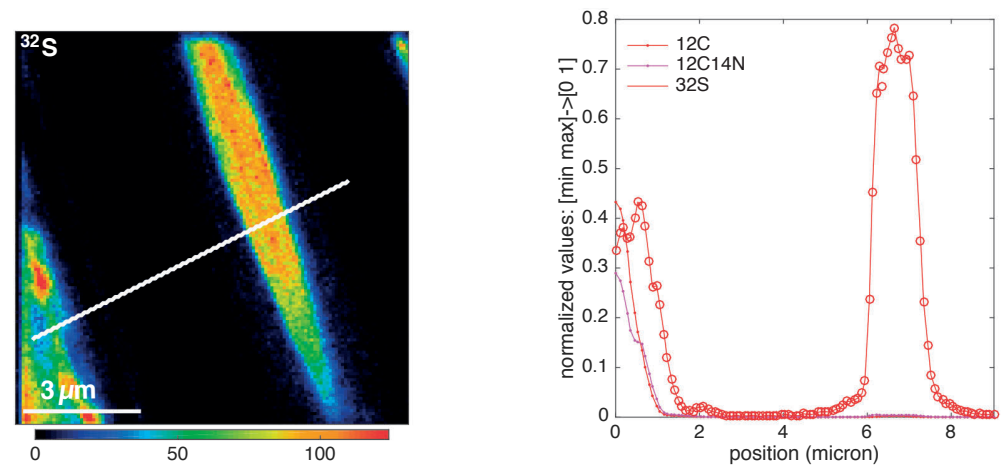

Figure S4.5: Sponge spicule with visible axial canal Shown are the ${ }^{32} \mathrm{~S}$ ion count ratio image and lateral profile of an incubated spicule with visible organic enrichment in the axial canal. In contrast to the resin, in which high counts of secondary ions 12C-, 12C14N- and 32S- were detected, only $32 \mathrm{~S}$ ion counts were detected in the axial canal of the sponge spicule. Thus, infill of axial canal with the resin is an unlikely explanation of the high $\mathrm{S}$ signal detected in the axial canal. 
a)

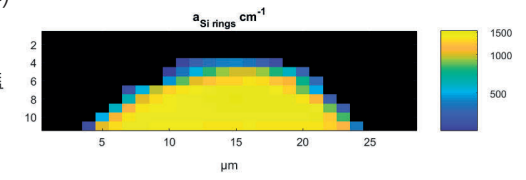

c)

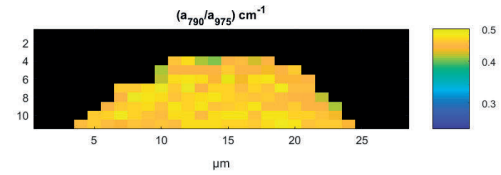

b)

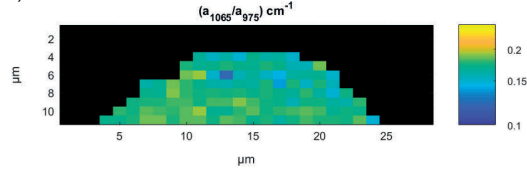

d)

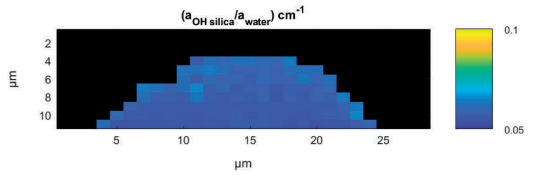

Figure S4.6: Raman 2D intensity maps of a spicule (a) Si-rings intensity (b) Si-O-Si/Si-OH (c) Inter tetrahedral $\mathrm{Si}-\mathrm{O}-\mathrm{Si} / \mathrm{Si}-\mathrm{OH}(\mathbf{d}) \mathrm{Si}-\mathrm{OH} / \mathrm{OH}$ water; The composite images show that, at this resolution, there are no clear patterns concerning the Si-OH distribution within the spicule. The maps show fairly homogenous ratio maps.

Table S4.1: Shapiro-Wilk test of normality - Crystalline silica incubations For these tests only the quartz crystals were compared as we did not measure a control for the sand grains. The assumption of normality could not be met for the incubated group. Transformation of the data did not change this. This is likely due to the low measurement number. A test over all the data does give a normal distribution

\begin{tabular}{lccc}
\hline & Statistic & df & Sig. \\
\hline Control quartz & 0.935 & 5 & .629 \\
${ }^{18}$ O-incubate quartz & 0.718 & 7 & .006 \\
All quartz & .902 & 15 & .102 \\
\hline
\end{tabular}

Table S4.2: Brown-Forsythe test for homoscedasticity

\begin{tabular}{lcccc}
\hline & Levene Statistic & df & df2 & Sig. \\
\hline $180 /(160+180))$ & 1.422 & 2 & 12 & .258 \\
SQRT(Fe/Si) & 2.825 & 75 & 56 & .004 \\
\hline
\end{tabular}

Table S4.3: Independent sample T-Test The difference between the control quartz and the quartz incubated in ${ }^{18} \mathrm{O}$-enriched seawater is not significant

\begin{tabular}{lccc}
\hline $\mathbf{t}$ & df & Sig. \\
\hline $180 /(160+180))$ & -2.103 & 10 & .062 \\
\hline
\end{tabular}


Table S4.4: Descriptive statistics - Spicule heating influence In section 3.5 we study the effect of heating on the ${ }^{18} \mathrm{O}$ atom fractions of spicules. Here we perform a test to see if this resulted in significant changes.

\begin{tabular}{lccc}
\hline $\mathbf{1 8 0} /(\mathbf{1 6 0 + 1 8 0 )}$ & Mean & Std. Deviation & $\mathrm{N}$ \\
\hline Pre-heating & $2.84 E-03$ & $1.30 E-04$ & 7 \\
Post-heating & $2.93 E-03$ & $3.13 E-04$ & 7 \\
\hline
\end{tabular}

Table S4.5: Pillai's Trace - Spicule heating influence

\begin{tabular}{cccccc}
\hline Value & $\mathbf{F}$ & Hypothesis df & Error df & Sig. & Partial Eta Squared \\
\hline 0.125 & $.856 b$ & 1 & 6 & 0.391 & 0.125 \\
\hline
\end{tabular}

Table S4.6: Shapiro-Wilk test of normality - DSi Three different treatments were maintained for the diatom incubation in the first three weeks. The supernatants of these tests were analysed for DSi content. The assumption of normality was met for the measurements of only one of the three treatments. However, this assumption was met for the total-dataset. Transformation did improve the normality significantly. The final result should be met with caution.

\begin{tabular}{lccc}
\hline & Statistic & df & Sig. \\
\hline${ }^{18}$ O-labelled natural SW & 0.887 & 10 & 0.156 \\
${ }^{18}$ O-labelled autoclaved SW & 0.759 & 12 & 0.003 \\
Natural Seawater & 0.844 & 12 & 0.031 \\
All treatments & 0.939 & 34 & 0.059 \\
\hline
\end{tabular}

Table S4.7: Levene's test of equality of error variances - DSi $(\mu \mathrm{mol} / \mathrm{L})$

\begin{tabular}{lcccc}
\hline & $\mathbf{F}$ & $\mathbf{d f}$ & $\mathbf{d f 2}$ & Sig. \\
\hline All groups & 0.992 & 2 & 31 & 0.382 \\
\hline
\end{tabular}

Table S4.8: ANGOVA analysis - DSi This analysis yields a non-significant interaction factor, indicating that the slopes are statistically the same. The treatment type was taken as a fixed factor and the time as a covariate. Note the results from the normality test in Table S6.

\begin{tabular}{lcccccc}
\hline Source & $\begin{array}{c}\text { Type III Sum } \\
\text { of Squares }\end{array}$ & df & Mean Square & F & Sig. & $\mathbf{h}^{2}$ \\
\hline Corrected Model & $22185.533 a$ & 5 & 4437.107 & 109.149 & 0.000 & 0.951 \\
Intercept & 15734.8 & 1 & 15734.8 & 387.063 & 0.000 & 0.933 \\
Type & 5764.193 & 2 & 2882.097 & 70.897 & 0.000 & 0.835 \\
Time & 12473.101 & 1 & 12473.101 & 306.828 & 0.000 & 0.916 \\
Type ${ }^{*}$ Time & 18.875 & 2 & 9.438 & 0.232 & 0.794 & 0.016 \\
Error & 1138.251 & 28 & 40.652 & & & \\
Total & 79154.671 & 34 & & & & \\
Corrected Total & 23323.784 & 33 & & & & \\
\hline
\end{tabular}

$* R^{2}=0.951$ (Adjusted $R^{2}=.942$ ) 


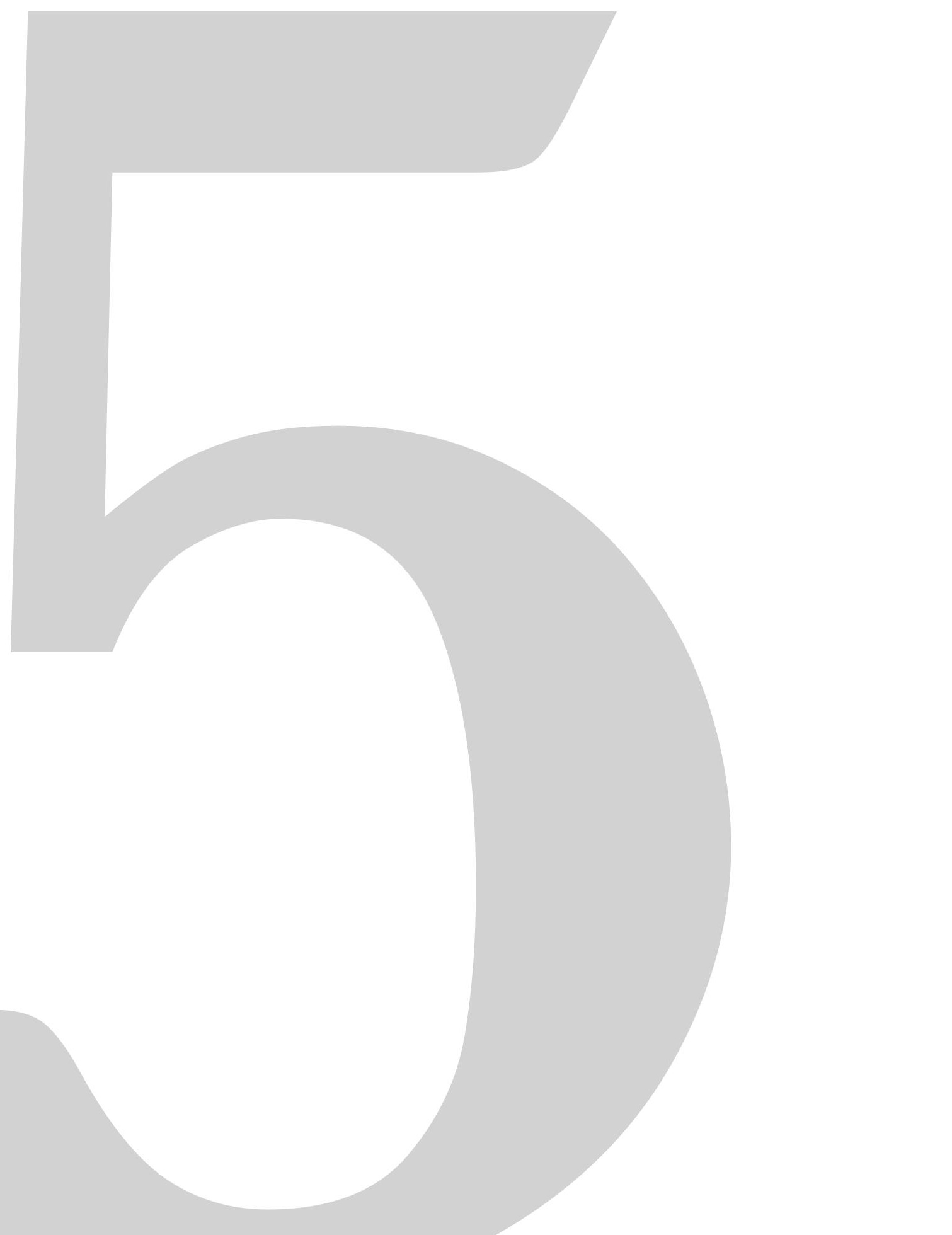




\title{
Chapter 5
}

\section{The influence of sediment diagenesis and aluminium-content on the secondary oxygen isotope exchange of diatom frustules}

\author{
Shaun P. Akse; Lubos Polerecky; Michiel V.M. Kienhuis and JackJ. Middelburg \\ Department of Earth Sciences, Utrecht University, PO Box 80021, 3508 TA Utrecht, The Netherlands
}

\begin{abstract}
The results from Chapter 4 of this thesis showed that diagenesis of $\delta^{18} \mathrm{O}_{\text {diatom }}$ already occurs in the water-column. Here we investigate whether overprinting of the $\delta^{18} \mathrm{O}$ signal in diatom frustules continues during different diagenetic phases in the sediment. A diatomaceous clay was incubated with ${ }^{18} \mathrm{O}$-enriched seawater during which fresh diatom detritus was placed at the sediment-water interface and at depth in the sediment. NanoSIMS analysis showed that the fresh diatom detritus and fossils frustules underwent significant ${ }^{18} \mathrm{O}$-enrichment. The degree to which this occurred varied; the fresh frustules placed at the sediment-water interface were most enriched followed by the fresh diatoms buried in the sediment and finally the fossil frustules. The potential role of $\mathrm{Al}$ as an inhibitor of secondary oxygen exchange was also studied. Concentrations of $\mathrm{Al}$ on the surface of the fossil frustules originating from the sediment did not reduce the extent of ${ }^{18} \mathrm{O}$-enrichment in the underlying silica. Incubations with various concentrations of dissolved $\mathrm{Al}$ showed that the addition of $\mathrm{Al}$ led to a significant increase in frustule $\mathrm{Al} / \mathrm{Si}$, homogenously distributed in the frustule valve, and significantly lowered the amount of ${ }^{18} \mathrm{O}$-enrichment. It is hypothesized that $\mathrm{Al}$ incorporated into the silica structure can slow down ${ }^{18} \mathrm{O}$ exchange while $\mathrm{Al}$ present as surface contaminants (clays or other aluminosilicates) has no inhibitory role.
\end{abstract}




\subsection{Introduction}

Studying changes in the oxygen isotope composition of various carbonate and silica remains is a common tool in paleoceanography as these changes are thought to reflect changes in the temperature and the isotope composition of the water in which they were formed (e.g. Rohling \& Cook, 1999; Zachos, 2001; Swann \& Leng, 2009). One of the potential targets of $\delta^{18} \mathrm{O}$ studies is diatomaceous silica which has been receiving increasing attention over the last few decades. This is due to the fact that the most commonly used material for oxygen isotope analysis, calcium carbonate, is absent in large parts of the ocean, hampering our understanding of changing climates. In order to complement the foraminiferal studies, isotopic signature studies in diatom frustules have been performed in both marine and lacustrine settings (see Leng and Barker, 2006; Swann and Leng, 2009). However, despite increasing interest, compared to the oxygen isotope proxy in for example foraminifera, the proxy in diatom frustules is still largely underdeveloped. This is in part due to persisting worries about possible diagenesis (DeMaster et al., 2003).

Concerns surrounding diagenetic influences exist due to the nature of the amorphous silica structure. While the structure of frustules (as well as other silicates) is composed predominantly of silica tetrahedrons, a less dense hydrous layer consisting of loosely bonded -Si-OH molecules (silanol groups) surround it (Gendron-Badou et al., 2003). This layer poses a problem for the proxy as the oxygen in the - $\mathrm{Si}-\mathrm{OH}$ molecules is known to rapidly exchange with oxygen in the surrounding water. As such, the oxygen signal of the layer is thought to represent a combination of signals, including the formation signal but also pore-water signals. Studies suggest that between $7 \%$ and $40 \%$ of the entire oxygen signal may originate post-deposition (Knauth, 1973; Labeyrie, 1979; Labeyrie and Juillet, 1982; Leng et al., 2001; Leng and Sloane, 2008; Swann et al., 2008). These concerns have been strengthened by observed differences in $\delta^{18} \mathrm{O}_{\text {diatom }}$ between surface-water diatoms and diatoms taken from deep-water traps and surface sediments (Schmidt et al., 2001). This disparity is particularly evident in the wide array of calculated silica-water fractionation factors (Fresh/recent diatom frustules: Brandriss et al., 1998; Dodd and Sharp 2010; Sedimentary diatomaceous silica: e.g. Juillet-Leclerc and Labeyrie 1987; Matheney and Knauth, 1989; Shemesh et al., 1992).

Studies looking at post-mortem diagenesis have suggested several active processes that may influence the $\delta^{18} \mathrm{O}$ signal of diatom frustules. These include post-depositional partial dissolution (Smith et al. 2016), the precipitation of secondary silica and addition of silanol groups and the condensation of silanol groups to siloxane (Schmidt et al., 2001; Dodd et al., 2012, 2017; Moschen et al., 2006, Rickert et al., 2002; Louciades et al., 2010). A result of these diagenetic processes is the decreasing reactivity of the frustules during maturation (Lewin, 1961). Silica condensation and subsequent reduction in silanol abundance has been suggested to be a main contributor of the observed decreasing reactivity, a process that is suggested to continue for about $10^{4}-10^{6}$ years (Dodd et al., 2017). This would mean that isotopic re-equilibration of oxygen could continue on geological time-scales.

Based on this research, it appears that the amount of available reactive sites (e.g., silanol groups) is a predominant factor controlling potential oxygen exchange. Next to the proposed maturation processes, studies have shown that the incorporation of metals can also strongly reduce the reactivity of frustules. In particular the presence and incorporation of aluminium (Al) in the silica structure has been linked to reducing reactivity and solubility (Van Bennekom et al., 1991; Dixit et al., 2001). This incorporation is driven by the presence of the reactive silanol groups which are excellent ligands for metals (Dixit \& Van Cappellen, 2002). The Al taken up 
during secondary processes, has been shown to originate from detrital materials and can become structurally incorporated into the silica framework by forming a new surface phase on the frustule (aluminosilicate coating) or by altering the existing mineral structure by replacing Si atoms (van Capellen \& Qiu, 1997; Dixit et al., 2001; Koning et al., 2007; Ren et al., 2013; Michalopoulos and Aller, 2004). The resulting deposition and incorporation of $\mathrm{Al}$ appears to take place rapidly on the time-scales of mere days or weeks (Koning et al., 2007). The formation of aluminosilicate anions can result in a negative charge, which repels the hydroxyl ions responsible for dissolution of the silica (Iler, 1973, 1979), thus protecting the frustule from dissolution and possibly also from secondary oxygen entering the structure.

In Chapter 4 of this thesis, we found that overprinting of the $\delta^{18} \mathrm{O}$ signal in diatom frustules can already take place in the water column on short time-scales (days to weeks). We observed such overprinting not only in diatom frustules but also in siliceous sponge spicules, another form of biogenic amorphous silica. These results showed that the final $\delta^{18} \mathrm{O}$ signal of fossil biogenic silica is at least a combination of different signals including the original surface water signal, the signal of the water column during sinking, and the signal of the sediment-water interface.

This study aims to investigate whether overprinting of the $\delta^{18} \mathrm{O}$ signal in diatom frustules continues similarly during the different diagenetic phases in the sediment: sediment-water interface, after burial and after maturation. In this study, fresh diatom detritus was folded into polycarbonate filters to create packets and placed on top of and in a siliceous ooze, after which natural or ${ }^{18} \mathrm{O}$-enriched seawater was added under anoxic conditions. The frustules from these packets, as well as the fossil frustules from the 40-60 $\mu \mathrm{m}$ fraction of the surrounding sediment, were extracted after two weeks and studied with nanoSIMS (Nanometer Secondary Ion Mass Spectrometer) analysis. In this way we were able to study oxygen exchange in diatom frustules at the sediment-water interface, after burial and after maturation. The potential influence of decreasing reactivity, due to metal incorporation, on secondary oxygen exchange was further studied by adding ${ }^{18} \mathrm{O}$-label and varying aluminium concentrations to natural seawater. With the nanoSIMS, we were able to study individual frustules and determine the relationships between $\mathrm{Al}$-concentrations and the exchange of oxygen.

\subsection{Materials \& Methods}

\subsubsection{Materials}

Two separate diatoms batches were cultured (Thalassiosira pseudonana and Thalassiosira weisflogii) at the Royal Netherlands Institute for Sea Research (NIOZ) by using an f/2 medium. The T.weisflogii strain (CCAP 1085/18) was provided by the culture collection of the NIOZ while the T.pseudonana strain (CAAP 1085/12) was acquired at the Culture Collection of Algae and Protozoans (CAAP). Cultures were grown in $300 \mathrm{~cm}^{3}$ tissue culture flasks at a temperature of $20{ }^{\circ} \mathrm{C}$ under a $16 / 8$ light/ dark cycle. A total of $2 \mathrm{~L}$ of medium per diatom species was produced and split into $500 \mathrm{~mL}$ containers for harvesting. After harvesting the frustules were freeze-dried (Telstar LyoQuest) and stored at $-20^{\circ} \mathrm{C}$. An aliquot of each $500 \mathrm{~mL}$ container was taken for cell count determination using flow cytometry. Before use, the frozen dry sample was diluted with $50 \mathrm{ml}$ of MilliQ $(18.2 \mathrm{M} \Omega \cdot \mathrm{cm}$ at $25^{\circ} \mathrm{C}$ ).

The sediment for this study was provided by Henko de Stigter (Netherlands Institute for Sea Research). This sediment is a diatomaceous clay originally collected in the Peru basin $\left(07^{\circ} 04.4^{\prime} \mathrm{S}\right.$ $088^{\circ} 27.8^{\prime} \mathrm{W}$ ) at $4150 \mathrm{~m}$ during the summer of 2015 . This sediment was the remainder of a boxcore 
after the top $20 \mathrm{~cm}$ had been removed. After collection the sediment was stored at $5{ }^{\circ} \mathrm{C}$. After being used for the sediment incubation experiment, the fossil diatom frustules from the $40-60 \mu \mathrm{m}$ size fraction of the sediment were also analysed with the nanoSIMS.

\subsubsection{Sediment incubation experiment}

For this experiment, a batch of the acquired sediment (see materials) and a beaker of natural seawater were placed in a glovebox for over 24 hours, in order to simulate anoxic bottom water conditions, and then mixed. For the mixing, the sediment-water ratio was kept at a 1:1 volume ratio. After the diatom pellets from the cultures were diluted with MilliQ, $0.1 \mathrm{~mL}$ of each diatom solution was pipetted onto a Nuclepore polycarbonate (PC) filter (3 $\mu \mathrm{m}$ pore size). These filters were folded several times to enclose the diatoms in the packet. A $1.5 \mathrm{~mL}$ Eppendorf vial was filled with $0.25 \mathrm{~mL}$ of the sediment-water mixture. One of the filter packets was placed in the tube and topped with another $0.25 \mathrm{~mL}$ of the sediment-water mixture, placing the packet at $\sim 1 \mathrm{~cm}$ depth. After another diatom packet was placed on the sediment, each sample tube was topped with a seawater mixture (1 mL natural seawater $(\mathrm{NSW})+0.1 \mathrm{~mL} 97 \% \mathrm{H}_{2}{ }^{18} \mathrm{O}$ resulting in ${ }^{18} \mathrm{O}$ atom fraction of $9 \%$ ). It was approximated that it took three days for the ${ }^{18} \mathrm{O}$-enriched water to diffuse through the sediment. The tightly closed vials were placed in an upright position in a water bath (to avoid potential rapid changes in the environment) and incubated for $336 \mathrm{hr}$. When ready to extract, the vials were removed from the glovebox. After this, the filters were removed and, using a filtration set-up, the contents of the filters were rinsed onto clean $0.2 \mu \mathrm{m}$ PC filters and left to dry in air. Finally, the remaining sediments were washed and sieved with $100,60 \& 40 \mu \mathrm{m}$ sieves. The $40-60 \mu \mathrm{m}$ size fraction was dried in a Petri dish for nanoSIMS analysis while the other fractions were disposed.

\subsubsection{Incubation experiment with added dissolved Al}

For this experiment, three seawater mixtures were prepared. For the control no additional Al was added to the natural seawater. For the other two seawater mixtures, $150 \mathrm{nM}$ and $500 \mathrm{nM} \mathrm{Al}$ were added (Inorganic ventures; $10032 \pm 32 \mu \mathrm{g} / \mathrm{mL}$ aluminium; 99.9981\% material purity; 7\% $\mathrm{HNO}_{3}$ matrix). For each experiment, $1 \mathrm{~mL}$ of the seawater solution was mixed with $0.1 \mathrm{~mL}$ of $97 \%$ $\mathrm{H}_{2}{ }^{18} \mathrm{O}$ in a $1.5 \mathrm{~mL}$ Eppendorf tube (resulting in ${ }^{18} \mathrm{O}$ atom fraction of $9 \%$ ). Diatoms (mixture of T.pseudonana \& T.weisflogii) were added to the incubation seawater in $0.01 \mathrm{~mL}$ aliquots. In a climatecontrolled room, the sample-containing Eppendorf tubes were put into Greiner tubes and placed, in a horizontal position, on a roller-table to keep the water moving and the material in suspension. At the end of the incubation, the samples were centrifuged for $10 \mathrm{~min}\left(\mathrm{RCF}=15411 \mathrm{~g}, 20^{\circ} \mathrm{C}\right)$. The material was rinsed with MilliQ and filtered unto a $0.2 \mu \mathrm{m}$ PC filter. The filter was then airdried and stored in a Petri dish at $15^{\circ} \mathrm{C}$.

\subsubsection{Sample preparation and NanoSIMS imaging}

To prepare the samples for nanoSIMS analysis, dried PC-filters with the diatom detritus were coated with a $12 \mathrm{~nm}$ Au-layer using a sputter coater (JOEL JFC-2300HR high-resolution fine coater, JEOL FG-TM20 thickness controller). The frustules received no additional treatment prior or after this step. The samples were then imaged with table-top SEM (JEOL JCM-6000PLUS NeoScope Benchtop SEM) operating at a $10-\mathrm{kV}$ accelerating voltage for target identification.

Nanoscale secondary ion mass spectrometry was performed with the nanoSIMS 50L instrument (Cameca) operated at Utrecht University. Electron multiplier detectors were set using a standard (SPI Supplies, 02757-AB 59 Metals \& Minerals Standard) to enable the detection of secondary ions ${ }^{12} \mathrm{C}_{-}^{-},{ }^{16} \mathrm{O}^{-},{ }^{18} \mathrm{O}^{-},{ }^{12} \mathrm{C}^{14} \mathrm{~N}^{-},{ }^{28} \mathrm{Si}^{-}$and ${ }^{27} \mathrm{Al}^{16} \mathrm{O}^{-}$with the $\mathrm{Cs}^{+}$primary ion beam. The 
oxygen isotopes and ${ }^{27} \mathrm{Al}^{16} \mathrm{O}$ are the main focus of research. The siliceous nature of the frustules can be identified with ${ }^{28} \mathrm{Si}$ and the organic signals $\left({ }^{12} \mathrm{C}\right.$ and $\left.{ }^{12} \mathrm{C}^{14} \mathrm{~N}\right)$ are used to identify the background filter and any remaining organic matter on the frustules. First, samples were placed in the airlock to reach vacuum levels of $10^{-8}$ mbar and heated to $\sim 50^{\circ} \mathrm{C}$ for at least 24 hours in order to remove any adsorbed water. After this the samples were moved to the vessel chamber and kept in vacuum $\left(10^{-9} \mathrm{mbar}\right)$ until analysis.

Prior to the analysis of each target diatom frustule, the measurement area was pre-sputtered with the primary ion beam (FCo current 20pA). For the fresh diatom frustules, low-energy implantation was used to implant Gs + ions into the surface of the frustules for better secondary ion yields without eroding the structure of the sample. For the fossil frustules, to reach stable secondary ion yields, pre-sputtering diaphragm settings were first set to D1-0, with C4y set to 50 bits to avoid detector overloading, and the pre-sputtering was monitored until the counts at detector \#3 (28Si) reached $\sim 170 \mathrm{kcps}$. This continued with the diaphragm switched to D1-1 until stable secondary ion counts were reached. After pre-sputtering, analysis was carried out in the imaging mode by rastering a high-energy $\mathrm{Cs}+$ beam $(16 \mathrm{keV}$, current at the sample surface of $0.5 \mathrm{pA}$, nominal beam size 50-150 nm) over a targeted area on the frustule (between $7 \times 7 \mu \mathrm{m}$ and $20 \times 20 \mu \mathrm{m}$ in size) and measuring the sputtered secondary ions at a resolution of $128 \times 128$ pixels and with dwell time of 1ms/pixel. The diaphragm and slit settings were D1-3, ES-3, AS-2, EnS-1. Analysis generally continued until the integrity of the frustules was lost as observed by strong mutations to the physical structural.

\subsubsection{NanoSIMS data processing}

NanoSIMS data were analysed with an updated version of the Matlab-based freeware software Look@NanoSIMS (Polerecky et al., 2012). An important step included the analysis of depth profiles. Specifically, in fresh frustules the depth profiles showed an increase in the ${ }^{18} \mathrm{O}$ atom fraction and a decrease in the ${ }^{27} \mathrm{Al}^{16} \mathrm{O} /{ }^{28} \mathrm{Si}$ ion count ratios during the first 50-200 planes (Supplementary Fig. S5.1). The initial increase in the ${ }^{18} \mathrm{O}$ atom fraction was attributed to sample preparation (rinsing with MilliQ), whereas the decrease in the ${ }^{27} \mathrm{Al}^{16} \mathrm{O} /{ }^{28} \mathrm{Si}$ ion count ratio was likely due to secondary ion yields not being completely stable after the initial low-energy implantation step of the measurement (additional details in Fig. S5.1). For the final dataset in Fig. 5.4, the initial 50-200 planes were therefore disregarded, and the data-points represent the mean and standard error over the planes where the ratios were stable. Furthermore, only the frustule valves were considered as these are commonly represented in the sedimentary archive.

Similar variation with depth was observed in fossil frustules for the ${ }^{27} \mathrm{Al}^{16} \mathrm{O}^{-} /{ }^{28} \mathrm{Si}$ ion count ratios but not for the ${ }^{18} \mathrm{O}$ atom fractions (Fig. S5.2). In contrast to fresh frustules, the fossil frustules were thoroughly pre-sputtered before each measurement, which was expected to stabilize the secondary ion yields (section 5.2.4). Thus, it is likely that the observed decline in the ${ }^{27} \mathrm{Al}^{16} \mathrm{O} /{ }^{28} \mathrm{Si}$ ion count ratio represented true variation of $\mathrm{Al} / \mathrm{Si}$ with depth in the silica frustule. The final dataset in Fig. 5.3 therefore shows statistics obtained from all planes measured in the individual frustules. Specifically, the data-points correspond to the median, while the error bars indicate the range between the 17-83 percentiles (i.e., encompassing $66 \%$ of values) divided by $\sqrt{ } \mathrm{N}$, where $\mathrm{N}$ is the number of planes measured. The error bars therefore represent the uncertainty with which the frustule-specific ${ }^{18} \mathrm{O}$ atom fractions and $\mathrm{Al} / \mathrm{Si}$ ratios were determined. Because the depth-variation in the ${ }^{18} \mathrm{O}$ atom fractions was only determined by the ion counts (Poisson statistics), the uncertainty is equal to the Poisson error, i.e., the analytical precision of the NanoSIMS measurement. In 
contrast, the uncertainty of the $\mathrm{Al} / \mathrm{Si}$ determination was mostly due to the variation of the ratio with depth while the Poisson error was negligible.

\subsection{Results}

\subsubsection{Post-mortem ${ }^{18} \mathrm{O}$-enrichment of diatom frustules in the sediment}

Representative images of ${ }^{18} \mathrm{O}$ atom fractions in diatom frustules clearly show that frustules incubated in ${ }^{18} \mathrm{O}$-enriched seawater had a significantly higher ${ }^{18} \mathrm{O}$ atom fraction than the control frustules (Fig. 5.1). Thus, placement of the frustules at or in the sediment did not stop secondary O-exchange. The fresh frustules placed at the sediment-water interface (Fig. 5.1b) were more enriched than those buried in the sediment (Fig. 5.1c), but this difference may have been partly due to the different exposure to ${ }^{18} \mathrm{O}$-enriched water linked to limited diffusive transport of the label through the sediment. Fossil frustules showed the lowest ${ }^{18} \mathrm{O}$-enrichment (Fig. 5.1d). In all frustules the ${ }^{18} \mathrm{O}$ enrichment was homogeneously distributed across the valve. However, in fresh frustules, where we could additionally observe the girdle (proteinaceous silica bands connecting the valves), the ${ }^{18} \mathrm{O}$ enrichment in the girdle was significantly lower than in the valve (Fig. 5.1b).

\subsubsection{Presence and influence of clay and other aluminosilicate contaminants on fossil frustules}

NanoSIMS measurements of fossil frustules revealed highly heterogeneous distribution of $\mathrm{Al}$ signals (measured as ${ }^{27} \mathrm{Al}^{16} \mathrm{O}^{-}$) on the frustule surface. In the SEM images (Fig. 5.2a) the larger Al-rich areas could be identified as particulate material blocking the pores or as pore narrowing. Thus, we attribute these Al-rich signals to surface-bound or pore-bound contamination, possibly clay particles or aluminosilicates.

The Al-rich contaminants did not appear to be enriched in ${ }^{18} \mathrm{O}$. Although pixels near the contaminants showed slightly higher ${ }^{18} \mathrm{O}$ atom fractions $(\sim 2.4 \mathrm{E}-3)$, this was likely the result of mixing of signals from the ${ }^{18} \mathrm{O}$-enriched silica and the ${ }^{18} \mathrm{O}$-unenriched contaminant. Importantly, the ${ }^{18} \mathrm{O}$ atom fraction in the $\mathrm{Al}$-rich areas increased with the measurement plane (i.e., depth) and reached the same level as in the surrounding silica when the Al-rich contaminant was sputtered away (Fig. 5.2c-f). This showed that the surface-bound particulate contamination did not prevent the underlying silica matrix from becoming enriched in ${ }^{18} \mathrm{O}$ during the incubation.

To ensure that the silica-specific ${ }^{18} \mathrm{O}$ atom fractions and $\mathrm{Al} / \mathrm{Si}$ ratios were not affected by the particulate contamination, the Al-rich areas were excluded from the final analysis of fossil frustules. The results showed that the fossil frustules incubated in ${ }^{18} \mathrm{O}$-enriched seawater had significantly greater ${ }^{18} \mathrm{O}$ atom fractions than the control frustules (Fig. 5.3). The ${ }^{18} \mathrm{O}$ atom fractions and $\mathrm{Al} / \mathrm{Si}$ ratios differed among individual frustules, but their correlation was not significant $(\mathrm{p}=.602$; Table S5.2). The sediment incubated in ${ }^{18} \mathrm{O}$-enriched seawater contained some foraminiferal shells, and these were not enriched in ${ }^{18} \mathrm{O}$ (Supplementary Fig. S5.3). 
a)

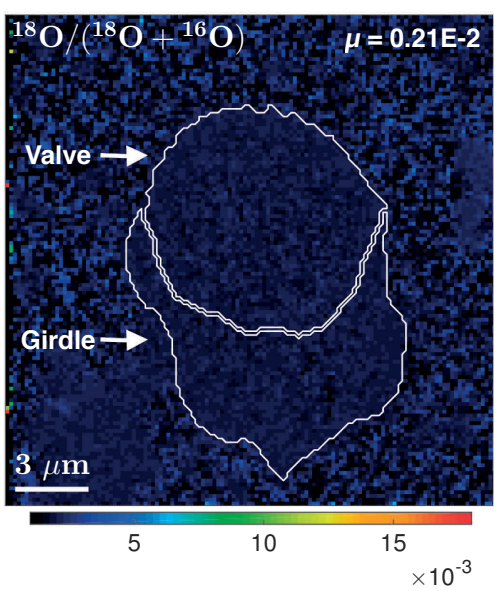

c)

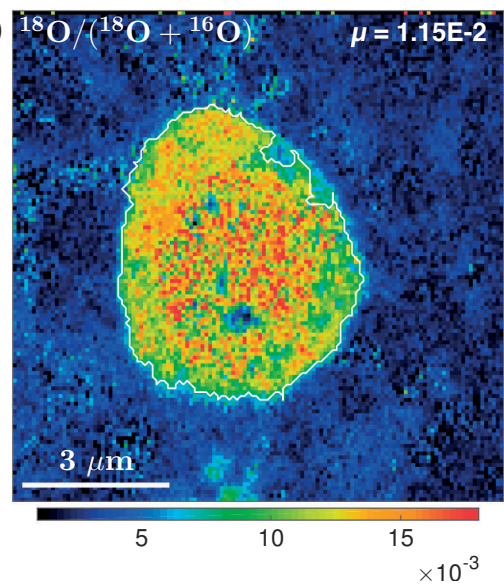

b)

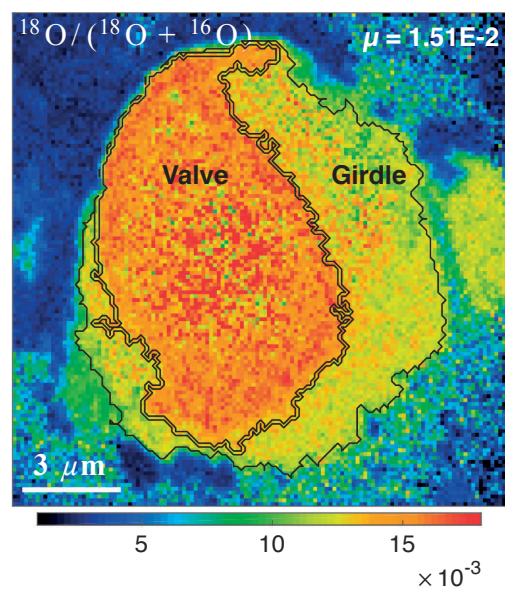

d)

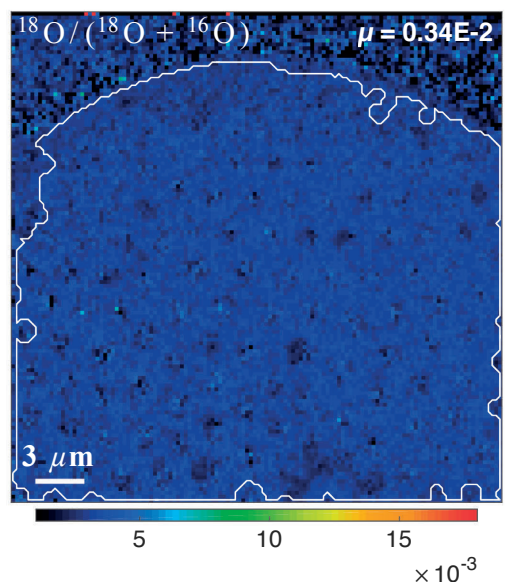

Figure 5.1: Imaging of ${ }^{18} \mathrm{O}$ atom fraction in diatom frustules incubated with ${ }^{18} \mathrm{O}$-enriched water. Shown are representative images of (a) a fresh control frustule incubated in natural seawater, (b) fresh frustule placed at the sediment-water interface and incubated in ${ }^{18} \mathrm{O}$-enriched seawater, $(\mathbf{c})$ fresh frustule placed at $\sim 1 \mathrm{~cm}$ depth in the sediment and incubated in ${ }^{18} \mathrm{O}$ enriched seawater, and (d) a fossil frustule from the 40-60 $\mu \mathrm{m}$ size fraction of the surrounding sediment and incubated in ${ }^{18} \mathrm{O}$ enriched seawater. ROI outlines mark the frustule valve and girdle. 
a)
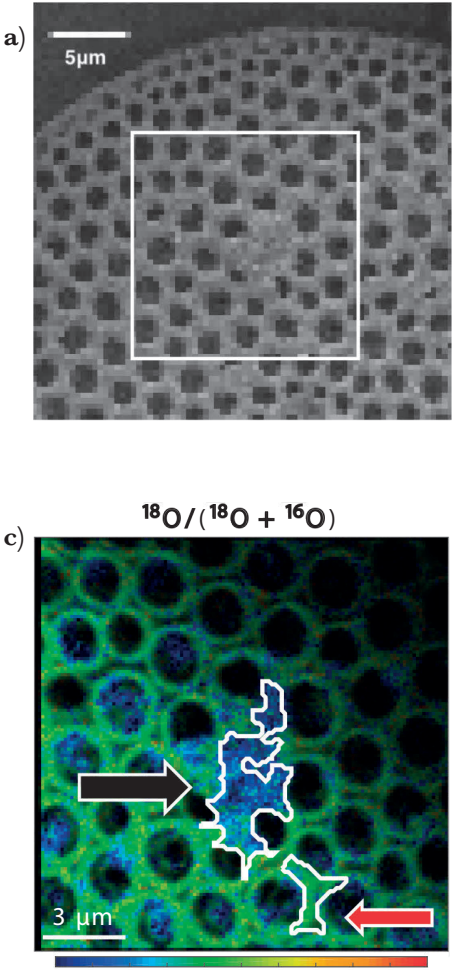

$2 \begin{array}{lllllllllll}2 & 2.2 & 2.4 & 2.6 & 2.8 & 3 & 3.2 & 3.4 & 3.6 & 3.8 & 4\end{array}$

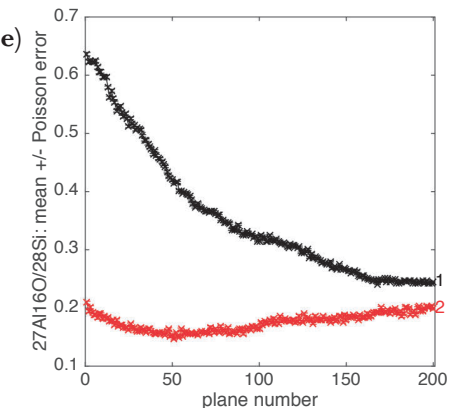

b)
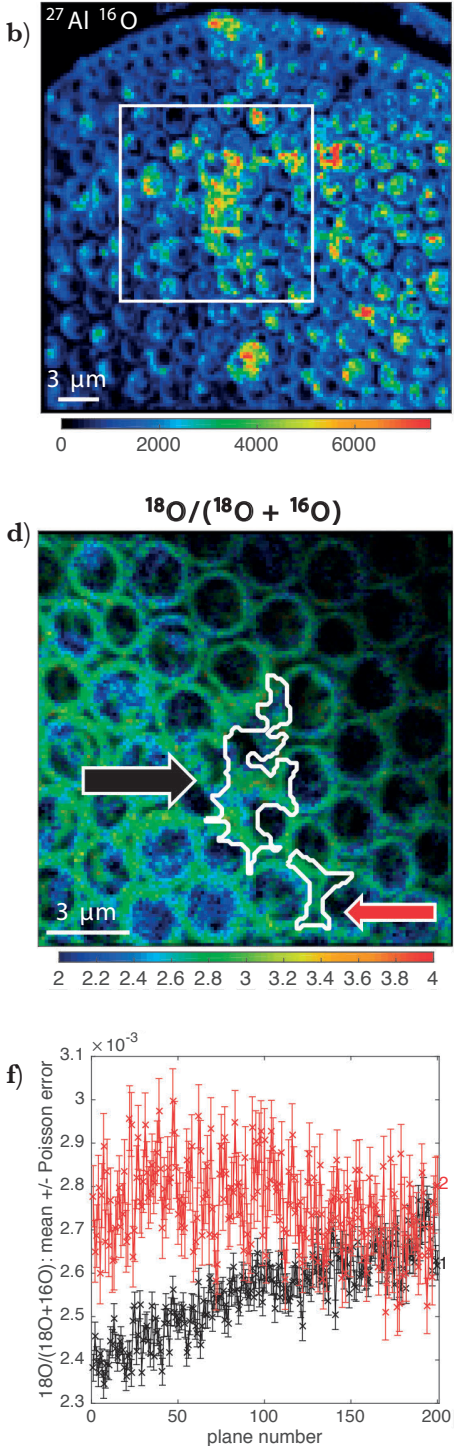

Figure 5.2: The presence and removal of $\mathrm{Al}$ on a fossil diatom frustule incubated in an ${ }^{18} \mathrm{O}$-enriched seawater. (a, b) SEM and ${ }^{27} \mathrm{Al}^{16} \mathrm{O}$ ion count image with white square showing the field-of-view for $\mathrm{c}-\mathrm{d}$; (c, $\mathbf{d}$ ) Ion count maps of the ${ }^{18} \mathrm{O}$ atom fraction. The hue of the image was modulated by the ${ }^{16} \mathrm{O}$ intensity image to suppress noise in the background (c) Planes 1-300; (d) Planes 1700-2000; Outlines mark the ROIs used for (e,f); (e, f) Shown are ratios as a function of the measurement plane (i.e. depth) accumulated in blocks of 10 planes. The lines represent the $\mathrm{Al}$-contaminant (black line) and the surrounding silica (red line) from (c,d). A decreasing $\mathrm{Al} / \mathrm{Si}$ trend is observed for the black line as the contaminant is sputtered away. As the contaminant is sputtered away, the ${ }^{18} \mathrm{O}$ atom fraction increases (black line) until the ratios are similar to the surrounding silica (red line). A very slight decline is observed in the ${ }^{18} \mathrm{O}$-enrichment of the silica, while a slow increase is observed in the ${ }^{27} \mathrm{Al} /{ }^{28} \mathrm{Si}$. Imaging showed that this pattern was present predominantly around the pores, suggesting that an $\mathrm{Al}$-presence from the pores may be influencing the silica data as more of the frustule is sputtered away. 


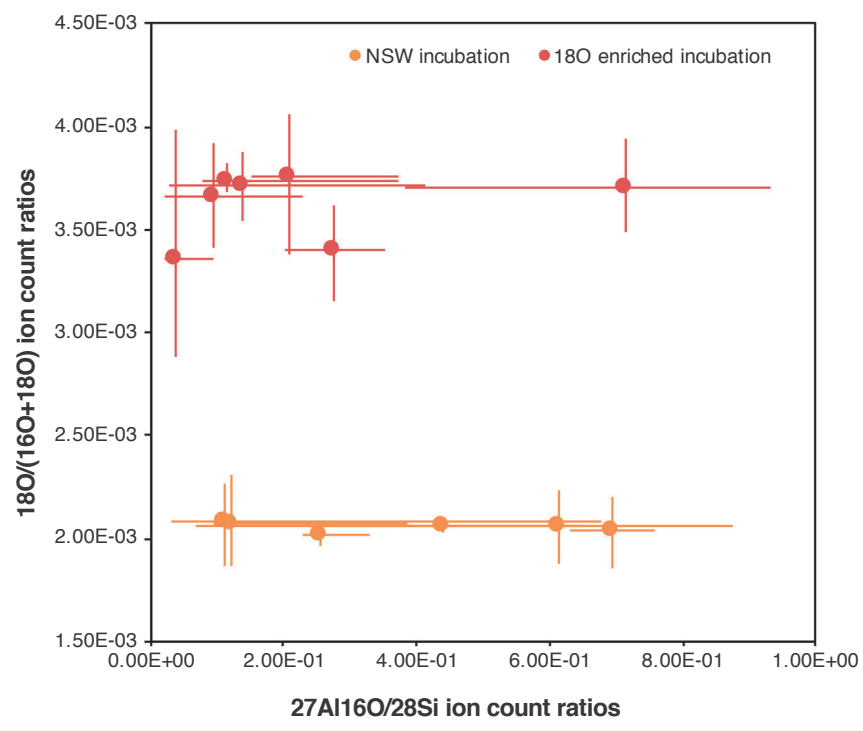

Figure 5.3: Graph showing ${ }^{27} \mathrm{Al}^{16} \mathrm{O} /{ }^{28} \mathrm{Si}$ ion count ratios (a proxy for molar $\mathrm{Al} / \mathrm{Si}$ ratio) vs ${ }^{18} \mathrm{O}$ atom fraction for fossil diatom frustules. Each datapoint corresponds to an individual frustule. See Methods for the meaning of error-bars.

\subsubsection{Influence of dissolved $\mathrm{Al}$ on ${ }^{18} \mathrm{O}$-enrichment in fresh frustules}

The influence of secondary Al-incorporation into the silica matrix on the rate of oxygen exchange between the frustule and seawater was studied in two ways. In the first approach, focusing on the impact of dissolved $\mathrm{Al}$ in the porewater (originating from the surrounding lithogenic particles), packets with fresh diatom detritus were placed at the sediment-water interface and at $1 \mathrm{~cm}$ depth in sediment and incubated in ${ }^{18} \mathrm{O}$-enriched seawater. Measurements of individual frustules revealed that the frustules at the sediment-water interface had significantly lower Al-content $(\mathrm{F}=24.7$; $\mathrm{p}<0.01$; Table S5.5) and significantly elevated ${ }^{18} \mathrm{O}$ atom fractions $(\mathrm{F}=23.0 ; \mathrm{p}<0.01$; Table S5.6) when compared with their counterparts that were buried in the sediment (Fig. 5.4a; Table 5.1).

In the second approach, fresh diatom frustules were incubated in the ${ }^{18} \mathrm{O}$-enriched natural seawater amended with dissolved $\mathrm{Al}$ (dAl; final concentrations of 0,150 , and $500 \mathrm{nM}$ ). The addition of $\mathrm{dAl}$ to the seawater was reflected in the $\mathrm{Al} / \mathrm{Si}$ values of the fresh frustules (Fig. 5.4b; Table 5.1), similar to results found in the literature (e.g., Koning et al., 2007). The addition of 500 $\mathrm{nM}$ dAl led to significantly higher 27Al16O/28Si ion count ratios in the frustules $(\mathrm{p}<0.05$; Table S5.10). NanoSIMS images showed that $\mathrm{Al}$ was distributed more homogenously through the frustule valve (Fig. S5.1), in contrast to the hotspots observed in the fossil frustules (Fig. 5.2). The high dAl treatment also significantly lowered the ${ }^{18} \mathrm{O}$ atom fractions of the frustules $(\mathrm{p}<0.05$; Table S5.12). The addition of $150 \mathrm{nM}$ also led to an increased $\mathrm{Al}$-content and reduced ${ }^{18} \mathrm{O}$-enrichment in the frustules, but the differences were not statistically significant (Tables S5.10 \& S5.12). 
a)

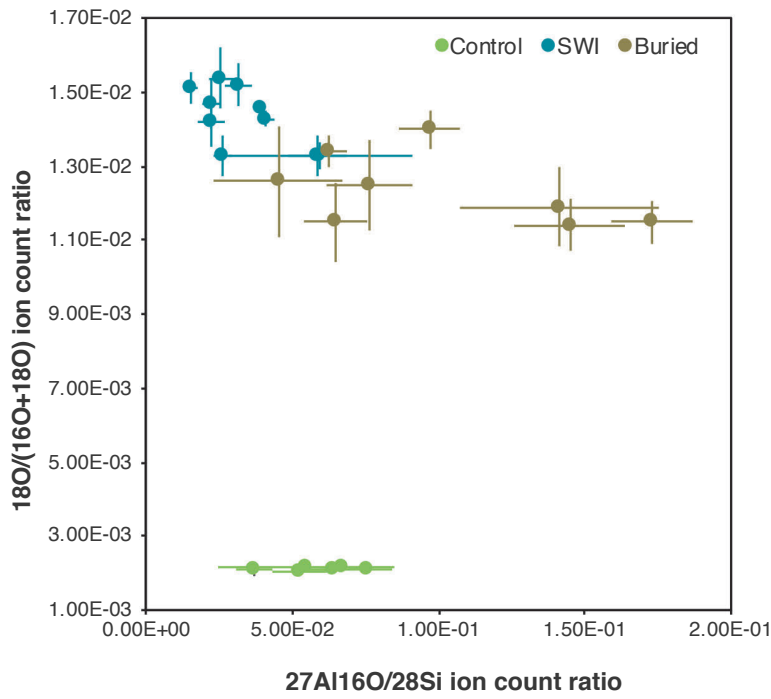

b)

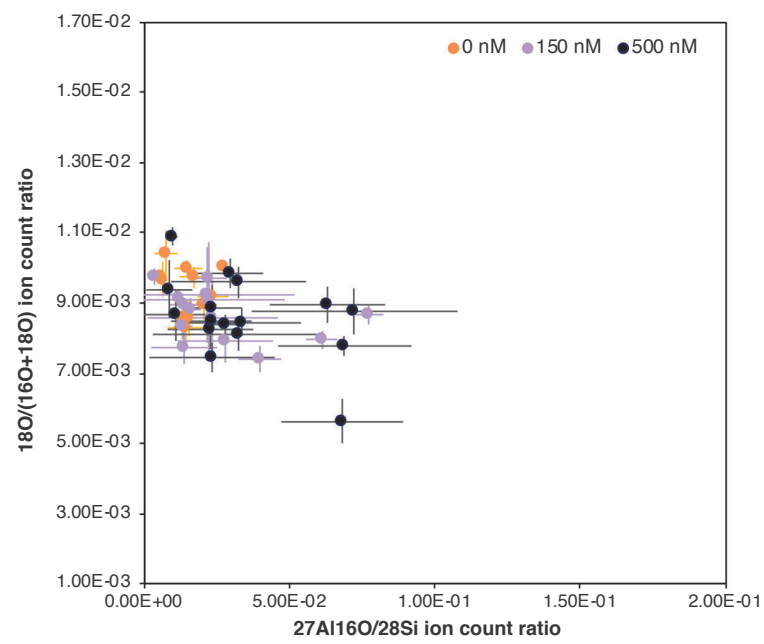

Figure 5.4: Silica-specific ${ }^{27} \mathrm{Al}^{16} \mathrm{O} /{ }^{28} \mathrm{Si}$ ion count ratios (a proxy for molar $\mathrm{Al} / \mathrm{Si}$ ratio) vs ${ }^{18} \mathrm{O}$ atom fractions in fresh diatom frustules. Shown are data from two incubation experiments in ${ }^{18} \mathrm{O}$-enriched seawater. (a) Frustules were placed at the sediment-water interface (SWI) or buried in the sediment for 2 weeks. The data for control frustules are also included. (b) Frustules were incubated for 1 week with different concentrations of dissolved $\mathrm{Al}(\mathrm{dAl})$. Additional data from the same incubation after 2 weeks are shown in Fig. S5.4. 
In addition to differences between the mean values, the comparison of individual frustules from the two incubation experiments revealed significant negative correlations between the $\mathrm{Al} / \mathrm{Si}$ ratios and the ${ }^{18} \mathrm{O}$ atom fractions (Fig. 5.4; $\mathrm{p}<0.05$; Table S5.15). The slopes of these correlations were not significantly different when comparing the incubations performed in the natural sediment and in the amended seawater (ANCOVA; $F_{\text {interaction }}=0.714 ; \mathrm{p}=0.402$; Table S5.16), indicating similar mechanisms. Furthermore, the coefficient of determination was greater for the sediment experiment $\left(\mathrm{R}^{2}=.644\right.$; Table $\left.\mathrm{S} 5.15\right)$ than the $\mathrm{dAl}$ experiment $\left(\mathrm{R}^{2}=.226\right.$; Table $\left.\mathrm{S} 5.15\right)$.

Table 5.1: Comparison of the chemistry of fresh diatom frustules incubated under different experimental settings. Shown are the means of ${ }^{27} \mathrm{Al}^{16} \mathrm{O} /{ }^{28} \mathrm{Si}$ ion count ratio (a proxy for molar $\mathrm{Al} / \mathrm{Si}$ ratio) and ${ }^{18} \mathrm{O}$ atom fraction. The 'Sediment' experiment compares the frustules placed at the sediment-water interface (SWI) and buried in the sediment for 2 weeks. The 'dAl' experiment compares the frustules incubated for 1-week with different concentrations of dissolved $\mathrm{Al}(\mathrm{dAl})$.

\begin{tabular}{lllll}
\hline Experiment & & $N$ & $27 A / 160 / 28 S i \pm S D$ & $180 /(160+180) \pm S D$ \\
\hline 'Sediment' & Control & 6 & $5.84 E-02 \pm 1.34 E-02$ & $2.11 E-03 \pm 0.03 E-03$ \\
& SWI $\left({ }^{18}\right.$ O-enriched seawater) & 10 & $3.41 E-02 \pm 1.53 E-02$ & $14.3 E-03 \pm 0.81 E-03$ \\
& Buried $\left({ }^{18}\right.$ O-enriched seawater) & 8 & $11.0 E-02 \pm 4.67 E-02$ & $12.4 E-03 \pm 0.96 E-03$ \\
\hline$' d A l '$ & OnM & 11 & $1.51 E-02 \pm 0.69 E-02$ & $9.38 E-03 \pm 0.69 E-03$ \\
& $150 \mathrm{nM}$ & 14 & $2.64 E-02 \pm 2.03 E-02$ & $8.66 E-03 \pm 0.72 E-03$ \\
& $500 \mathrm{nM}$ & 16 & $3.44 E-02 \pm 2.16 E-02$ & $8.59 E-03 \pm 1.15 E-03$ \\
\hline
\end{tabular}

\subsection{Discussion}

In Chapter 4, it was shown that biogenic silica undergoes a rapid resetting of the O-isotope signature when incubated in ${ }^{18} \mathrm{O}$-enriched seawater (Fig. 5.5). This led to the question if these post-mortem processes would continue when diatom detritus moves from the water column into the sediment, as studies have shown that the reactivity of the frustules decreases dramatically as a consequence of maturation during ageing (Dixit et al., 2001; Lewin, 1961 and others). In this chapter, we continued our study of early diagenetic processes on the O-isotope signature of frustules. More specifically, we looked at oxygen exchange during different stages of diagenesis and the role of $\mathrm{Al}$ as a potential inhibitor.

All the measured diatom frustules showed significant ${ }^{18} \mathrm{O}$ enrichment after having been incubated in ${ }^{18} \mathrm{O}$-enriched seawater, meaning that even in the sediments the oxygen signal of the frustules can be quickly altered by the oxygen signal of the surrounding water. However, the degree to which this occurred, differed. The strongest enrichment of ${ }^{18} \mathrm{O}$, in this study, was observed in the frustules placed at the sediment-water interface. These generally also had lower $\mathrm{Al}$-content than their counterparts that were buried in the sediment. In addition to the study of fresh diatom detritus, fossil frustules from the surrounding diatomaceous clay were also studied. Proof of frustule maturation is found in the higher Al-content when compared to the fresh frustules (Fig. S5.5; Gehlen et al., 2002; Van Capellen et al., 2002; Moschen et al., 2006) and the lack of hydroxyl groups during Raman measurements on these frustules in Chapter 4. Despite this more mature structure, the fossil frustules also showed an enrichment in ${ }^{18} \mathrm{O}$ albeit less strong than 
the fresh frustules. The absence of a water signal in the frustules after having spent time in the vacuum, rules out water in the frustule as a potential source of the oxygen signal. Instead, observed ${ }^{18} \mathrm{O}$-enrichment of fossil frustules together with the absence of -OH in the Raman signal (Chapter 4, Figure 4.7b) suggests that even with limited reaction sites, the silica of the fossil frustules is susceptible to diagenetic overprinting of the oxygen signal.

As mentioned, decreasing ${ }^{18} \mathrm{O}$-enrichment is likely linked to increasing maturation. In this study, we studied whether this corresponded with Al-content of the frustule; a common marker for biogenic silica diagenesis. The distribution of $\mathrm{Al}$ was different in the fossil frustules than the fresh frustules. In the valves of the fresh frustules, the increased Al-content was generally homogenously distributed (Fig. S5.1) whilst the fossil frustules showed an abundance of Al-hotpots (Fig. 5.2). The observed high $\mathrm{Al}$-content in the frustules buried in the sediment is in correspondence with the literature. It has been shown that $\mathrm{dAl}$ in the porewaters, originating from lithogenic particles, is higher and can be readily deposited on frustules post-mortem as aluminosilicates or can become structurally incorporated into the silica framework (van Capellen \& Qiu, 1997; Dixit et al., 2001; Koning et al., 2007; Ren et al., 2013; Michalopolous \& Aller, 2003).

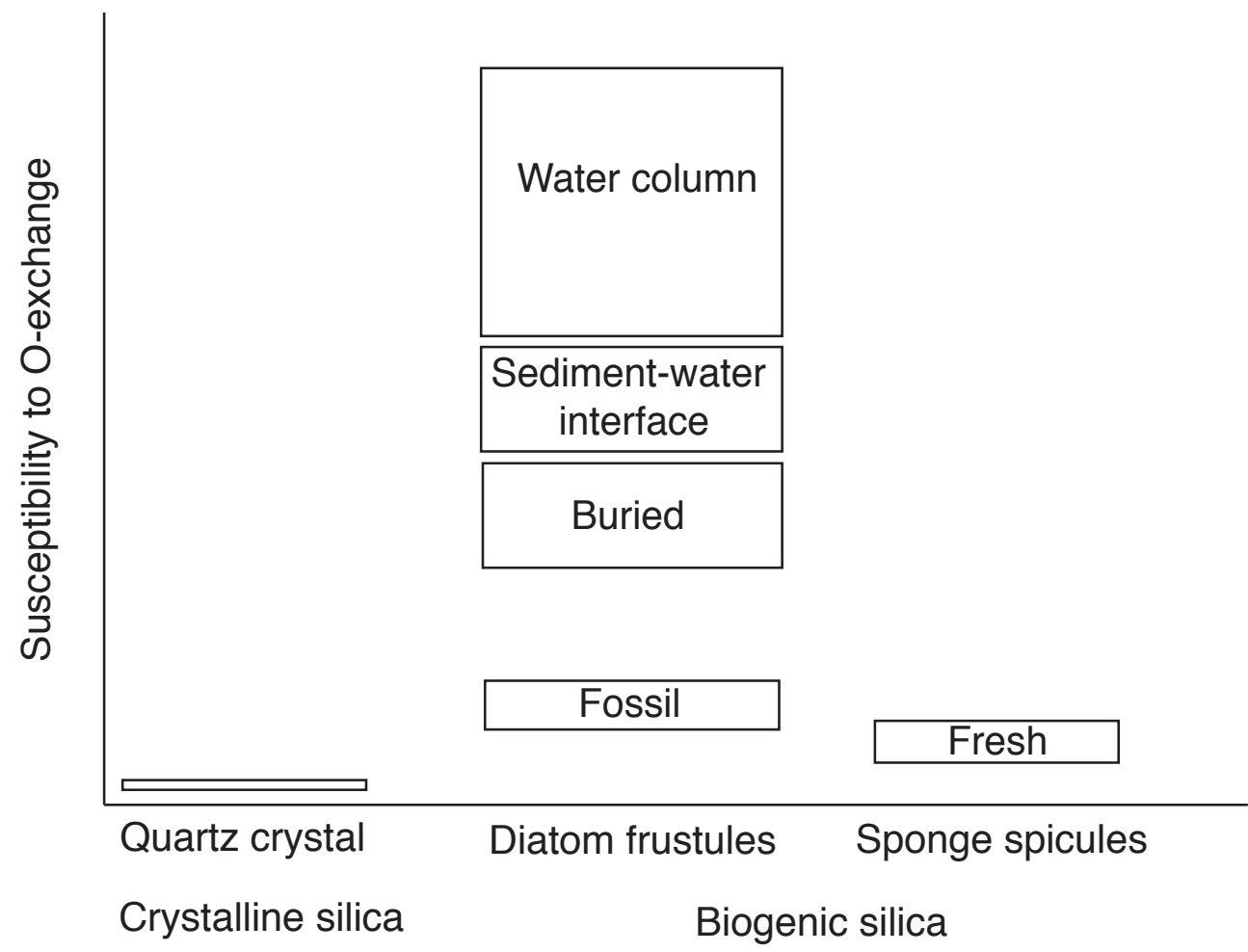

Figure 5.5: Conceptualization of the potential of different silica forms to become enriched in ${ }^{18} \mathrm{O}$

After being transported through the water column, diatom detritus will reach the sediment-water interface. Here the frustule will remain until they are buried and preserved over geological timescales. These processes lead to increasing maturity of the frustules. 
Some of the larger Al-hotspots on the fossil frustules could be identified in the SEM image by a narrowing or blocking of the pores suggesting either clay or other aluminosilicate deposits. Detailed spatial analysis of these $\mathrm{Al}$ contaminants allowed us to sputter some of them away, exposing the silica underneath. The silica underneath this surface contaminant had identical ${ }^{18} \mathrm{O}$-enrichment as the other areas of the 'clean' silica structure, unlike the contaminant itself which did not display ${ }^{18} \mathrm{O}$-enrichment. These results show that aluminosilicate presence does not inhibit post-mortem alterations to the isotopic oxygen signature of diatom frustules. Moreover, these results show how the application of imaging techniques such as the nanoSIMS can be advantageous, as even small contributions of the contaminants can have significant influences on the isotope values (Brewer et al., 2008).

The fresh diatom detritus showed a more homogenous distribution of $\mathrm{Al}$ and ${ }^{18} \mathrm{O}$ through the frustule valves (Fig. S5.1). The lower Al-content and higher ${ }^{18} \mathrm{O}$ atom ratios of the frustules placed at the sediment-water interface relative to the buried frustules may due to the higher availability of $\mathrm{Al}$ in the sediment and the diffusion gradient (estimated to be three days) of the ${ }^{18} \mathrm{O}$-enriched water into the sediment. However, it may also be that the reduced ${ }^{18} \mathrm{O}$-enrichment in the buried frustules confirms earlier observations that the transition of diatom detritus from the water column into the sediment results in decreasing reactivity due to interactions with metals in the pore water (Dodd et al., 2017, Rickert et al., 2002; Loucaides et al., 2010; Zhuravlev et al., 2000).

To further investigate the role of metals, an additional experiment was performed by placing frustules in ${ }^{18} \mathrm{O}$-enriched seawater with varying concentrations of $\mathrm{dAl}$. In this approach, other potential contributing factors are removed and only the effect of $\mathrm{Al}$ is studied. As the presence of $\mathrm{Al}$ was homogenously distributed over the frustule valve, the increasing $\mathrm{Al} / \mathrm{Si}$ with increasing $\mathrm{dAl}$ most likely reflected the structural incorporation of $\mathrm{Al}$ (when a silicon atom is substituted by an $\mathrm{Al}$ (III) ion (Ren et al., 2013)). Increasing the Al-content of the water resulted in significantly reduced ${ }^{18} \mathrm{O}$ atom fractions, suggesting an inhibitory role of diagenetically incorporated Al. The interaction between $\mathrm{Al}$-content and ${ }^{18} \mathrm{O}$-enrichment was statistically the same, suggesting similar mechanisms. However, the low to moderate coefficients of determination between $\mathrm{Al}$-content and ${ }^{18} \mathrm{O}$-enrichment suggest that the metal-content cannot be the only factor controlling the exchange of ${ }^{18} \mathrm{O}$.

\subsection{Conclusion}

After previously having observed that the oxygen signal in biogenic silica can be rapidly overprinted post-mortem in the water column (Fig. 5.5), this study shows that this process continues in the sediment. Frustules placed at the sediment-water interface and buried in the sediment both underwent significant ${ }^{18} \mathrm{O}$-enrichment. Fossil diatom frustules were also significantly influenced by secondary oxygen presence albeit to a lesser degree. This confirms previous studies reporting a decreasing reactivity of the frustules during maturation. However, this study also shows that despite the absence of silanol groups, the oxygen signal of fossil frustules may still be influenced over geological time scales. The role of $\mathrm{Al}$ in this process was also studied and the results show that $\mathrm{Al}$ can reduce ${ }^{18} \mathrm{O}$-exchange, but this depends on the form in which the $\mathrm{Al}$ is present. The $\mathrm{Al}$ seemingly incorporated into the silica structure appears to slow ${ }^{18} \mathrm{O}$-exchange while surface contaminants (clays or other aluminosilicates) do not. 


\section{Supplementary figures}

a)

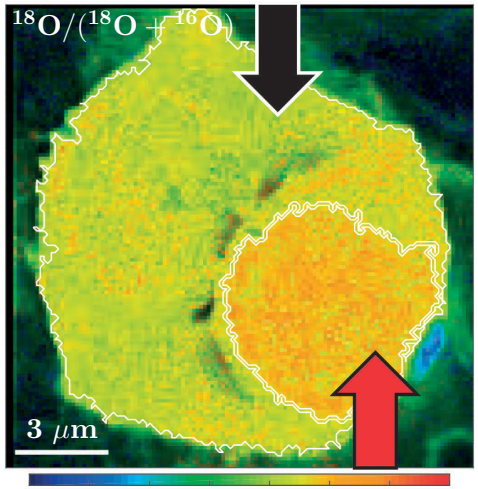

$\begin{array}{llllll}0 & 0.0020 .0040 .0060 .008 & 0.01 & 0.0120 .014\end{array}$
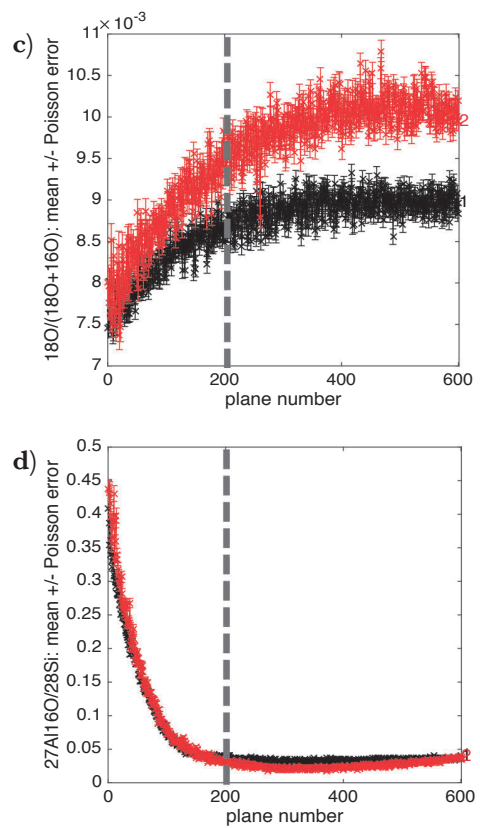

e)

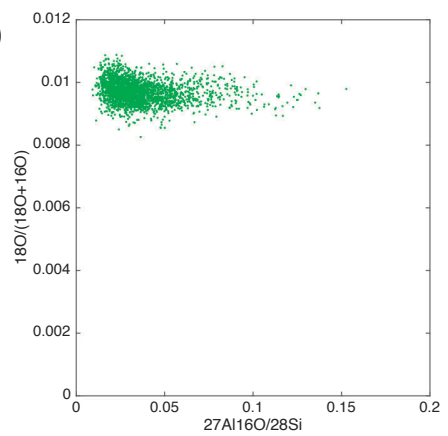

b)

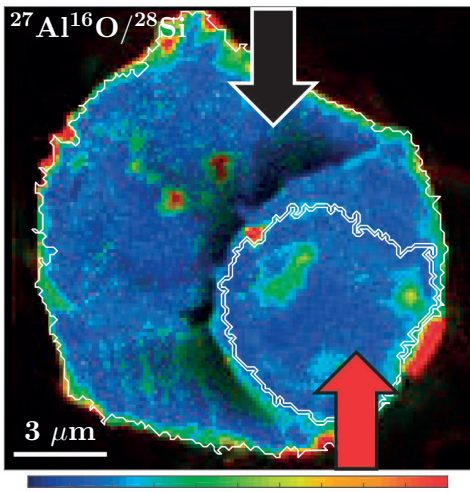

$0 \quad 0.020 .040 .060 .080 .10 .120 .140 .160 .180 .2$

Figure S5.1: NanoSIMS analysis of a fresh frustule incubated in ${ }^{18} \mathrm{O}$-enriched seawater with added dAl (500nM) Frustule valve (Red arrow \& profile) and girdle (Black arrow and profile) are defined as ROIs; (a-b) Ion ratio map with white lines distinguishing the valve and girdle a) The valve has homogenous ${ }^{18} \mathrm{O}$ atom fractions but higher than the girdle b) The frustule shows an increase in $\mathrm{Al}$ throughout but a few high $\mathrm{Al}$ areas can be observed. Clay contamination cannot be the source as the frustule was not in direct contact with the sediment. (c-d) Ratios as a function of the measurement plane (i.e., depth), shown for two areas on the frustule. Dotted line marks potential start of the accumulation if unstable planes are removed (Red and black data points correspond to areas pointed to by the red and black arrows in panel a) and b); c) ${ }^{18} \mathrm{O} /\left({ }^{16} \mathrm{O}+{ }^{18} \mathrm{O}\right)$; d $){ }^{27} \mathrm{Al}^{16} \mathrm{O} /{ }^{28} \mathrm{Si}$;. The initial increase in panel c) is attributed to sample preparation where the frustules were rinsed with MilliQ which has no ${ }^{18} \mathrm{O}$-enrichment. The rapid decrease in panel d) is attributed to the LE-implantation approach which led to initial unequal sputtering rates; (e) ${ }^{27} \mathrm{Al}^{16} \mathrm{O} /{ }^{28} \mathrm{Si}$ vs ${ }^{18} \mathrm{O}$ atom ratio for each pixel in the valve $\mathrm{ROI}$, showing that there is no correlation between the two ratios despite the presence of small high-Al areas. 

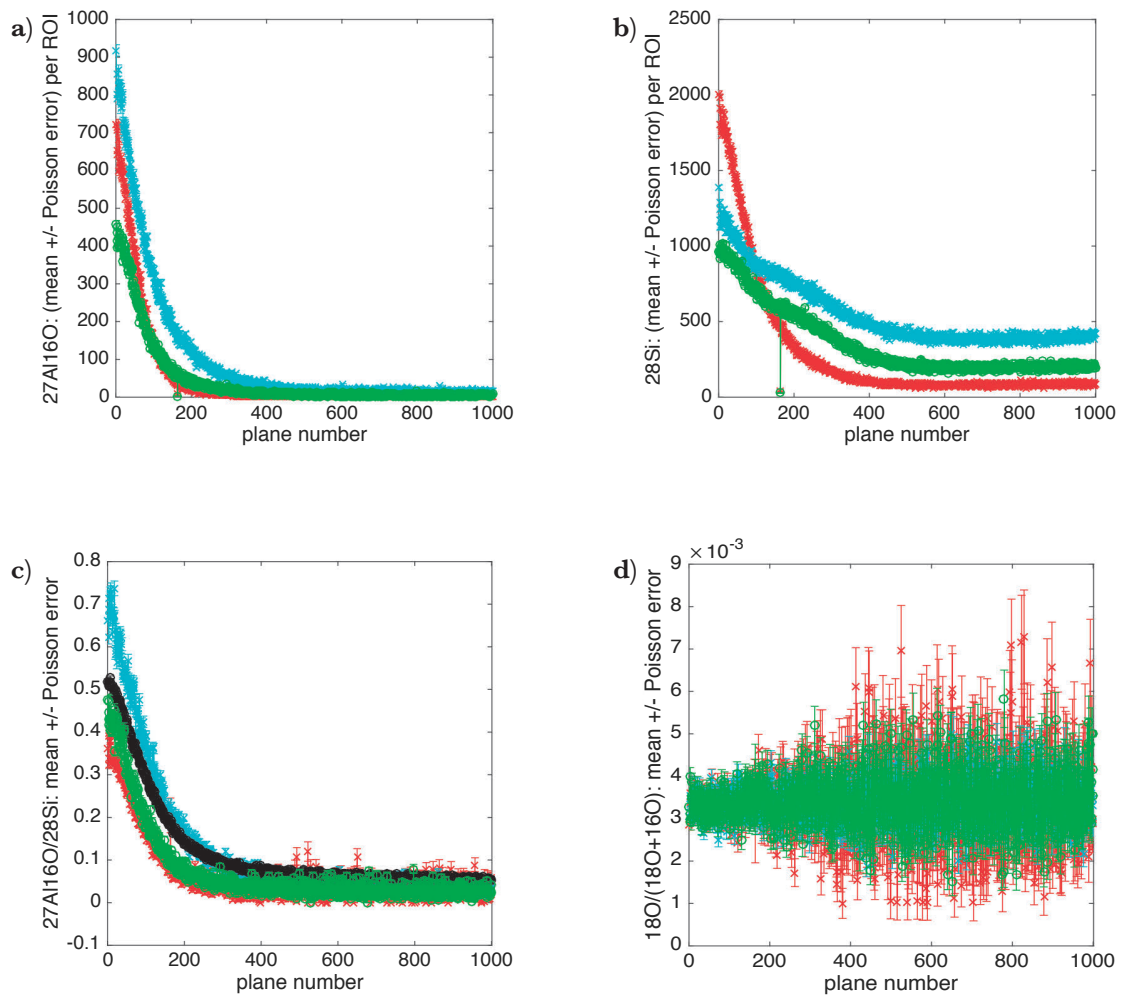

Figure S5.2: Depth profiles of an ${ }^{18} \mathrm{O}$-enriched fossil frustule Shown are depth profiles of three different ROIs on a single frustule. All ROIs were placed on 'clean' silica between pores. In the fossil frustules, we observed stable ${ }^{18} \mathrm{O} /\left({ }^{16} \mathrm{O}+{ }^{18} \mathrm{O}\right)$ values but unstable sputtering profiles for $\mathrm{Al} / \mathrm{Si}$. However, the rate of change does decrease as the measurement progresses. The $\mathrm{Al} / \mathrm{Si}$ profile appears to differ from Fig. S5.1d as the decrease is less rapid. The sputtering profiles suggest that the $\mathrm{Al} / \mathrm{Si}$ signal is a combination of true signals and analytical bias due to unstable sputtering profile. As all measured frustules received the same thorough pre-sputtering treatment, we decided to maintain the ratios of the whole measurement. In this way we do not exclude data which may be representative of a true decreasing $\mathrm{Al} / \mathrm{Si}$ ratio. To account for the unstable profiles, the error bars in Fig. 5.3 represent the error due to depth. 

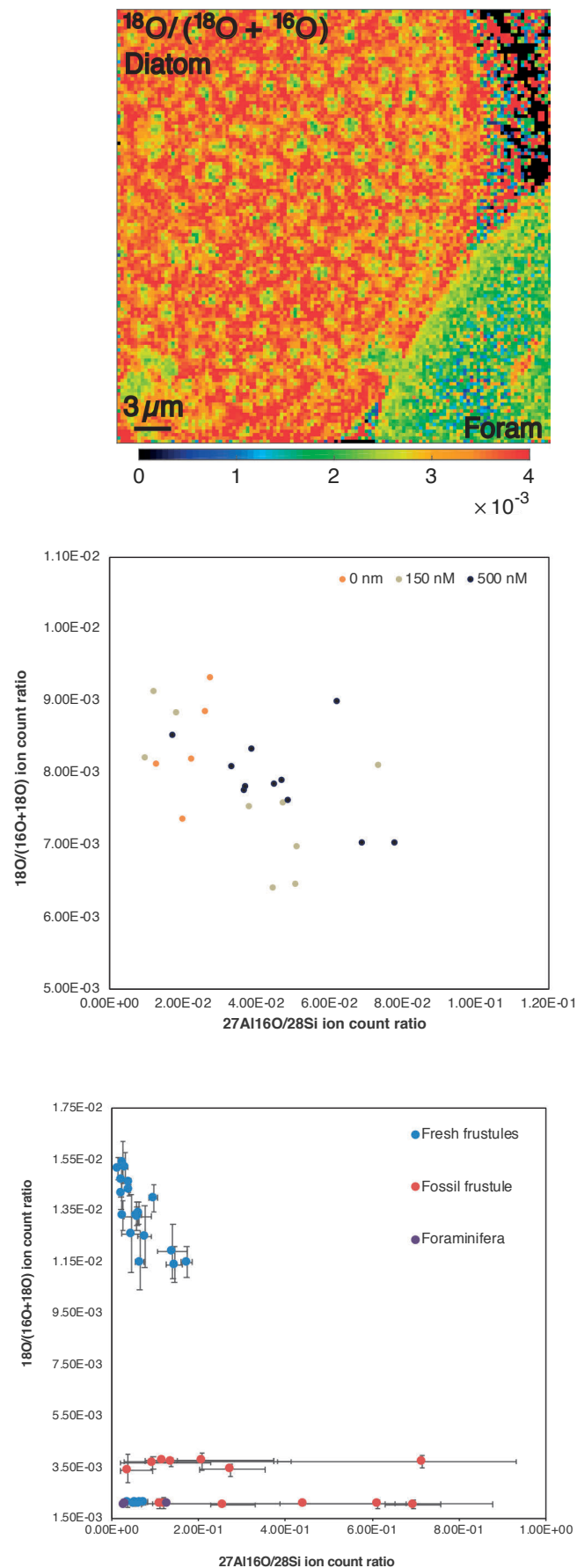

Supplementary figure S5.3: Degree of oxygen exchange between fossil frustules and foraminifera in a ${ }^{18} \mathrm{O}$-enriched sample Despite the dominance of siliciclastic fossil tests, several isolated foraminifera were also spotted in the ${ }^{18} \mathrm{O}$-enriched sample. The foraminifera did not show signs of ${ }^{18} \mathrm{O}$-enrichment. The contrast is particularly clear in the measurement that encompassed both. This lack of enrichment is fully consistent with common use of foraminiferal $\delta^{18} \mathrm{O}$ in paleoceanography.

Supplementary figure S5.4: ${ }^{18}$ O-enrichment of fresh diatoms incubated with varying additions of dAl for 2 weeks The results for the one-week incubation are presented in the main text. The same experiment was also performed for a two-week incubation. This two-week experiment confirmed the patterns of the one-week incubation but included less data points. For the total dataset the correlation between ${ }^{18} \mathrm{O}$ atom fractions and Al-content was significant (Table S5.13).

Supplementary figure S5.5: Graph showing ${ }^{27} \mathrm{Al}{ }^{16} \mathrm{O} /{ }^{28} \mathrm{Si}$ ion count ratios (a proxy for molar $\mathrm{Al} / \mathrm{Si}$ ratio) vs. ${ }^{18} \mathrm{O}$ atom fraction for all collected datapoints within one incubation. The data shown is from the sediment-incubation experiment. Each datapoint is an individual specimen; For the frustules incubated in ${ }^{18} \mathrm{O}$-enriched water, the fresh frustules showed a significantly greater enrichment than the fossil frustules. The $\mathrm{Al} / \mathrm{Si}$ ratios were lower for the fresh samples compared to the fossil samples. Also, the $\mathrm{Al} / \mathrm{Si}$ ratios of the fossil samples showed significantly greater variability. Note that for the fresh frustules, unstable planes were removed. This did not strongly alter the mean but did reduce the error. 
Supplementary Table S5.1: Ion count ratios after two weeks in ${ }^{18} \mathrm{O}$ enriched seawater and various

dAl treatments Shown are the means and SD for the various treatments. With increased dAl input, the $\mathrm{Al} / \mathrm{Si}$ ratio increases in the frustules. The addition of $\mathrm{Al}$ also decreases the $18 \mathrm{O}$ enrichment, although there is very little difference between the $150 \mathrm{nM}$ and $500 \mathrm{nM}$ treatments.

\begin{tabular}{lccccc}
\hline Treatment & $\mathbf{N}$ & 27Al160/28Si & $\mathbf{\pm S D}$ & $\mathbf{1 8 0 / ( 1 6 0 + 1 8 0 )}$ & \pm SD \\
\hline OnM & 11 & $2.20 E-02$ & $0.58 E-02$ & $8.36 E-03$ & $0.75 E-03$ \\
$150 \mathrm{nM}$ & 14 & $3.78 E-02$ & $2.12 E-02$ & $7.68 E-03$ & $0.97 E-03$ \\
$500 \mathrm{nM}$ & 16 & $4.69 E-02$ & $1.73 E-02$ & $7.89 E-03$ & $0.58 E-03$
\end{tabular}

Supplementary Table S5.2: Bivariate analysis for fossil frustules incubated in ${ }^{18} \mathrm{O}$-enriched seawater This analysis shows that the correlation between ${ }^{18} \mathrm{O}$ atom fraction and Al-content in the fossil frustules (Fig. 5.4). No significant correlation is detected.

\begin{tabular}{lccccc}
\hline & $\mathrm{N}$ & $\begin{array}{c}\text { Pearson } \\
\text { correlation }\end{array}$ & Sig. & Slope & $\mathbf{R}^{2}$ \\
\hline Fossil frustules & 7 & .241 & .602 & $1.7 E-4$ & .058 \\
\hline
\end{tabular}

Supplementary Table S5.3: Shapiro-Wilk test of normality for fresh frustules in the sedimentincubation experiment The results from frustules placed at the sediment-water interface were compared with frustules placed at a depth of $1 \mathrm{~cm}$ in the sediment. In order to comply with the assumptions of normality and homoscedasticity, the data was transformed with a natural $\log$ function.

\begin{tabular}{llccc}
\hline & & Statistic & df & Sig. \\
\hline Ln(Al/Si) & Sediment-waterinterface & .950 & 10 & .671 \\
& Buried & .941 & 8 & .621 \\
\hline $\operatorname{Ln}(180 /[160+180])$ & Sediment-waterinterface & .881 & 10 & .135 \\
& Buried & .898 & 8 & .275 \\
\hline
\end{tabular}

Supplementary Table S5.4: Brown-Forsythe test for homoscedasticity. Test for fresh frustules in the sediment-incubation experiment. Assumption is met.

\begin{tabular}{lcccc}
\hline & Levene Statistic & df & df2 & Sig. \\
\hline $\operatorname{Ln}(\mathrm{Al} / \mathrm{Si})$ & 0.152 & 1 & 16 & .702 \\
$\operatorname{Ln}(180 /[160+180])$ & 0.967 & 1 & 16 & .340 \\
\hline
\end{tabular}


Supplementary Table S5.5: ANOVA analysis $\mathbf{L n}(\mathbf{A 1} / \mathbf{S i})$ Test for fresh frustules in the sediment-incubation experiment. There are significant differences between the samples at the sediment-water interface and buried at $1 \mathrm{~cm}$ in the sediment.

\begin{tabular}{lcccccc}
\hline Source & $\begin{array}{c}\text { Type III Sum of } \\
\text { Squares }\end{array}$ & df & Mean Square & F & Sig. & 2 \\
\hline Corrected Model & $5.104 a$ & 1 & 5.104 & 24.273 & 0.000 & 0.603 \\
Intercept & 152.74 & 1 & 152.74 & 726.336 & 0.000 & 0.978 \\
Type & 5.104 & 1 & 5.104 & 24.273 & 0.000 & 0.603 \\
Error & 3.365 & 16 & 0.21 & & & \\
Total & 169.465 & 18 & & & & \\
Corrected total & 8.469 & 17 & & & & \\
\hline
\end{tabular}

$* R^{2}=0.603$ (Adjusted $R^{2}=.578$ )

Supplementary Table S5.6: ANOVA analysis $\operatorname{Ln}(\mathbf{1 8 O} /[16 \mathrm{O}+180])$ Test for fresh frustules in the sedimentincubation experiment There are significant differences between the samples at the sediment-water interface and buried at $1 \mathrm{~cm}$ in the sediment.

\begin{tabular}{lcccccc}
\hline Source & $\begin{array}{c}\text { Type III Sum of } \\
\text { Squares }\end{array}$ & df & Mean Square & F & Sig. & 2 \\
\hline Corrected Model & $.101 a$ & 1 & 0.101 & 23.018 & 0.000 & 0.59 \\
Intercept & 331.994 & 1 & 331.994 & 75880.982 & 0.000 & 1 \\
Type & 0.101 & 1 & 0.101 & 23.018 & 0.000 & 0.59 \\
Error & 0.07 & 16 & 0.004 & & & \\
Total & 335.015 & 18 & & & & \\
Corrected total & 0.171 & 17 & & & & \\
\hline
\end{tabular}

$* R^{2}=0.590$ (Adjusted $R^{2}=.564$ )

Supplementary Table S5.7: Shapiro-Wilk test of normality for the incubation experiment with varying dAl addition The results from the different treatments were compared. In order to comply with the assumptions of normality and homoscedasticity, the data was transformed with a natural log function.

\begin{tabular}{llccc}
\hline & & Statistic & df & Sig. \\
\hline Ln(Al/Si) & No Al addition & .921 & 11 & .325 \\
& $150 \mathrm{nM}$ & .943 & 14 & .463 \\
& $500 \mathrm{nM}$ & .912 & 16 & .132 \\
\hline Ln(180/[160+180]) & No Al addition & .927 & 11 & .377 \\
& $150 \mathrm{nM}$ & .957 & 14 & .678 \\
& $500 \mathrm{nM}$ & .889 & 16 & .054 \\
\hline
\end{tabular}


Supplementary Table S5.8: Brown-Forsythe test for homoscedasticity. Test for fresh frustules in the dAl incubation experiment. Assumption is met

\begin{tabular}{lcccc}
\hline & Levene Statistic & df & df2 & Sig. \\
\hline $\operatorname{Ln}(\mathrm{Al} / \mathrm{Si})$ & 0.369 & 2 & 38 & 0.694 \\
$\operatorname{Ln}(180 /[160+180])$ & 1.985 & 2 & 38 & 0.151 \\
\hline
\end{tabular}

Supplementary Table S5.9: ANOVA analysis Ln(A1/Si) Test for fresh frustules in the dAl incubation experiment. There are significant differences between the different treatments with varying dAl

\begin{tabular}{lcccccc}
\hline Source & $\begin{array}{c}\text { Type III Sum } \\
\text { of Squares }\end{array}$ & df & Mean Square & F & Sig. & 2 \\
\hline Corrected Model & $3.545 a$ & 2 & 1.773 & 4.03 & 0.000 & 0.175 \\
Intercept & 614.209 & 1 & 614.209 & 1396.494 & 0.000 & 0.974 \\
Type & 3.545 & 2 & 1.773 & 4.03 & 0.000 & 0.175 \\
Error & 16.713 & 38 & 0.44 & & & \\
Total & 634.879 & 41 & & & & \\
Corrected total & 20.258 & 40 & & & & \\
\hline
\end{tabular}

$* R^{2}=0.175$ (Adjusted $R^{2}=.132$ )

Supplementary Table S5.10: Tukey HSD Post-HOG test Ln(A1/Si) Test for fresh frustules in the dAl incubation experiment. The tests showed that only the $500 \mathrm{nM}$ treatment led to significantly increased Al-content in the frustules

\begin{tabular}{llccccc}
\hline (I) dAl content & (J) dAl content & $\begin{array}{c}\text { Mean Difference } \\
(\mathrm{I}-\mathrm{J})\end{array}$ & Std. Error & Sig. & \multicolumn{2}{c}{$\begin{array}{c}\text { 95\% Confidence Interval } \\
\text { Lower Upper }\end{array}$} \\
\hline No Al addition & $150 \mathrm{nM}$ & -0.4202 & 0.26721 & 0.27 & -1.0719 & 0.2315 \\
& $500 \mathrm{nM}$ & -.7372 & 0.25976 & $\mathbf{0 . 0 1 9}$ & -1.3707 & -0.1037 \\
\hline $150 \mathrm{nM}$ & No Al addition & 0.4202 & 0.26721 & 0.27 & -0.2315 & 1.0719 \\
& $500 \mathrm{nM}$ & -0.317 & 0.2427 & 0.401 & -0.9089 & 0.2749 \\
\hline $500 \mathrm{nM}$ & No Al addition & .7372 & 0.25976 & 0.019 & 0.1037 & 1.3707 \\
& $150 \mathrm{nM}$ & 0.317 & 0.2427 & 0.401 & -0.2749 & 0.9089 \\
\hline
\end{tabular}


Supplementary Table S5.11: ANOVA analysis $\operatorname{Ln}(18 \mathrm{O} /[16 \mathrm{O}+18 \mathrm{O}])$ Test for fresh frustules in the dAl incubation experiment. There are significant differences between the different treatments with varying $\mathrm{dAl}$

\begin{tabular}{lcccccc}
\hline Source & $\begin{array}{c}\text { Type III Sum } \\
\text { of Squares }\end{array}$ & df & Mean Square & $\mathbf{F}$ & Sig. & $\mathbf{h}^{2}$ \\
\hline Corrected Model & $.002 a$ & 2 & 0.001 & 3.596 & 0.037 & 0.159 \\
Intercept & 0.026 & 1 & 0.026 & 75.881 & 0 & 0.666 \\
Type & 0.002 & 2 & 0.001 & 3.596 & 0.037 & 0.159 \\
Error & 0.013 & 38 & 0 & & & \\
Total & 0.044 & 41 & & & & \\
Corrected total & 0.015 & 40 & & & &
\end{tabular}

$* R^{2}=0.159$ (Adjusted $R^{2}=.115$ )

Supplementary Table S5.12: Tukey HSD Post-HOG test $\operatorname{Ln}(18 \mathrm{10} /[16 \mathrm{O}+180])$ Test for fresh frustules in the $\mathrm{dAl}$ incubation experiment. The tests showed that only the $500 \mathrm{nM}$ treatment led to significantly reduced ${ }^{18} \mathrm{O}$ atom ratios in the frustules

\begin{tabular}{llccccc}
\hline (I) dAl content & (J) dAl content & $\begin{array}{c}\text { Mean Difference } \\
(\mathrm{I}-J)\end{array}$ & Std. Error & Sig. & $\begin{array}{c}95 \% \text { Confidence Interval } \\
\text { Lower Upper }\end{array}$ \\
\hline No Al addition & $150 \mathrm{nM}$ & $-1.12 E-02$ & $7.40 E-03$ & 0.294 & $-2.93 E-02$ & $6.81 E-03$ \\
& $500 \mathrm{nM}$ & $-1.9293 E-02$ & $7.19 E-03$ & 0.028 & $-3.68 E-02$ & $-1.75 E-03$ \\
\hline $150 \mathrm{nM}$ & No Al addition & $1.12 E-02$ & $7.40 E-03$ & 0.294 & $-6.81 E-03$ & $2.93 E-02$ \\
& $500 \mathrm{nM}$ & $-8.05 E-03$ & $6.72 E-03$ & 0.462 & $-2.44 E-02$ & $8.34 E-03$ \\
\hline $500 \mathrm{nM}$ & No Al addition & $1.9293 E-02$ & $7.19 E-03$ & 0.028 & $1.75 E-03$ & $3.68 E-02$ \\
& $150 \mathrm{nM}$ & $8.05 E-03$ & $6.72 E-03$ & 0.462 & $-8.34 E-03$ & $2.44 E-02$ \\
\hline
\end{tabular}

Supplementary Table S5.13: Shapiro-Wilk test of normality for the comparison between the slopes of the two experiments. This test was performed to see if the data, from the two experimental setups, have a common relationship between ${ }^{18} \mathrm{O}$ atom fraction and $\mathrm{Al}$-content. Possibly indicating similar driving factors. In order to comply with the assumptions of normality and homoscedasticity, the data was transformed with a natural log function.

Unfortunately, for the $\mathrm{dAl}$ experiment this could not be met. However, this test was met for the individual treatments (Table S5.6).

\begin{tabular}{llccc}
\hline & & Statistic & df & Sig. \\
\hline Ln(Al/Si) & Sediment experiment & .965 & 18 & .696 \\
& dAl experiment & .972 & 41 & .398 \\
\hline $\operatorname{Ln}(180 /[160+180])$ & Sediment experiment & .928 & 18 & .176 \\
& dAl experiment & .915 & 41 & .005
\end{tabular}


Supplementary Table S5.14: Levene's test of equality of error variances For comparison between slopes.

\begin{tabular}{lcccc}
\hline & $F$ & df & df2 & Sig. \\
\hline All groups & 2.579 & 1 & 57 & 0.113 \\
\hline
\end{tabular}

Supplementary Table S5.15: Bivariate analysis for slopes of two incubations experiments with fresh frustules. This analysis shows that the correlation between ${ }^{18} \mathrm{O}$ atom fraction and $\mathrm{Al}$-content is significant in both experiments. Note that the test was performed on natural-log transformed data

\begin{tabular}{lccccc}
\hline Experiment & $\mathbf{N}$ & $\begin{array}{c}\text { Pearson } \\
\text { correlation }\end{array}$ & Sig. & Slope & $\mathbf{R}^{2}$ \\
\hline Sediment & 18 & -.802 & 0.000 & -.11 & .644 \\
$d A l(1$ week) & 41 & -.516 & 0.001 & -.08 & .226 \\
$d A l(2$ week) & 25 & -.494 & 0.012 & -.09 & 0.244 \\
\hline
\end{tabular}

Supplementary Table S5.16: ANGOVA analysis for the two experimental set-ups The slope of the sediment experiment was compared with that of the $\mathrm{dAl}$ (1 week). The 1-week experiment was chosen due to the higher measurement count. The test shows that while the intercepts vary, the slopes of the two groups are statistically the same. A cautionary note is that the test for normality was not met for one group (Table S5.12).

\begin{tabular}{|c|c|c|c|c|c|c|}
\hline Source & $\begin{array}{l}\text { Type III Sum } \\
\text { of Squares }\end{array}$ & df & Mean Square & $F$ & Sig. & $\mathbf{h}^{2}$ \\
\hline Corrected Model & $2.485 a$ & 3 & 0.828 & 102.431 & 0.000 & .848 \\
\hline Intercept & 50.26 & 1 & 50.26 & 6216.089 & 0.000 & .991 \\
\hline Type & 0.086 & 1 & 0.086 & 10.694 & 0.002 & .163 \\
\hline Time & 0.231 & 1 & 0.231 & 28.589 & 0.000 & .342 \\
\hline Type * Time & 0.006 & 1 & 0.006 & 0.714 & 0.402 & .013 \\
\hline Error & 0.445 & 55 & 0.008 & & & \\
\hline Total & 1255.089 & 59 & & & & \\
\hline Corrected Total & 2.929 & 58 & & & & \\
\hline
\end{tabular}

$* R^{2}=0.848$ (Adjusted $\left.R^{2}=.840\right)$ 


$$
6
$$




\title{
Chapter 6
}

\section{The application of high-resolution imaging techniques to biogenic silica - Evaluation of sample preparation methods and analytical approaches}

\author{
Shaun P. Akse, Michiel V.M. Kienhuis, JackJ. Middelburg and Lubos Polerecky \\ Department of Earth Sciences, Utrecht University, PO Box 80021, 3508 TA Utrecht, The Netherlands
}

\begin{abstract}
The elemental and isotopic composition of biogenic silica has tremendous paleoproxy potential, and in the last few decades, the chemical compositions of, in particular, diatom frustules and sponge spicules have been receiving increased attention. As diatom frustules are an order of magnitude smaller than popular proxy targets such as foraminiferal tests, the increased interest in frustule chemistry can also be linked to the development of high spatial resolution analytical techniques. Here, we evaluate the application of high-resolution techniques to biogenic silica and discuss the various sample preparation techniques, analytical and data processing steps that we have undertaken to obtain the data presented in this thesis. Both the successful and unsuccessful attempts are evaluated, which will hopefully aid future researchers interested in unravelling the many mysteries still surrounding the chemistry of diatom frustules.
\end{abstract}




\section{1}

Introduction

At the start of this thesis, not much was known on the use of nanoSIMS to image and analyse biogenic silicates. Moreover, the nanoSIMS at Utrecht University was rarely used with the duoplasmatron $\mathrm{O}^{-}$source. Because of this lack of experience and expertise, some pioneering method development and testing was needed. A variety of different analytical approaches have been attempted, some were successful and some not. The successful approaches led, eventually, to the results presented in this thesis, but behind these results lies a treasure trove of failure. Ultimately, however, these failures can also be considered successes as they have helped us to further our understanding of biogenic silica. We continue the study on biogenic silica because it has tremendous paleoproxy potential. In areas where the traditional material for chemical paleoproxies (carbonate) is absent, biogenic silica is often abundant. Due to this, diatom frustule and sponge spicule chemistry has been receiving increased attention in recent decades. So far, most diatom frustule proxies have focused on bulk methods such as ICP-MS (Osterholz et al., 2014) and GC-IRMS (Robinson et al., 2004) in order to get sufficient precision. The application of highresolution imaging techniques to diatom frustules and other forms of biogenic silica for proxy use has been limited but can aid us in understanding the signals originating from the frustules.

In this chapter, we will discuss the various sample preparation techniques, analytical and data processing steps that we have undertaken to obtain the data presented in this thesis. First, we evaluate different techniques applied to prepare samples for nanoSIMS analysis. Next we discuss the application of nanoSIMS analysis to biogenic silica. In this section considerations for different sample types are examined, and we provide a number of suggestions that might be helpful for a reader interested in the study of biogenic silica by NanoSIMS. This is followed by recommendations surrounding nanoSIMS data processing of diatom frustules analyses. Finally, the application of other analytical imaging methods to frustules including Raman spectroscopy and transmission electron microscopy are discussed.

\subsection{Sample preparation}

Sample preparation is key to successful analytical imaging techniques such as nanoSIMS. How a sample is optimally prepared for analysis depends on several factors including the type and goals of the analysis, the type of sample (e.g., diatom frustule or sponge spicule), the source of the sample (e.g. sediment or water column), and the degree of fossilization. In this section we will discuss these different parameters and evaluate which sample preparation methods are best suited for nanoSIMS analysis of biogenic silica.

\subsubsection{Requirements for analysis}

Every analytical method has some requirements concerning the nature of the sample. For nanoSIMS, the most important sample requirements are vacuum compatibility and a flat surface. Vacuum compatibility was not an issue for any of the sample preparation methods used in this thesis. The flat surface requirement has proven more difficult to tackle. Due to the design of the ion optics, the nanoSIMS is known to be sensitive to sample topography, which can strongly influence the secondary ion yield. Internal normalization to a major element is often applied to account for changes in secondary ion yields throughout the measurement, but this does not solve individual element-specific responses to topography. There are several approaches that may be applied to reduce the potential role of topography. These can be sought in sample preparation and 
data processing; both are discussed in the succeeding sections. Another prerequisite for reliable nanoSIMS measurements is good conductivity of the sample surface in order to avoid charging effects from the polarity of the primary ion beam. At the nanoSIMS facility in Utrecht this is facilitated by sputter-coating a thin conductive layer of gold (e.g. 2-20 nm) onto the sample. Finally, it is also important to keep in mind, during sample preparation, what signals may be coming from the background and how these may interfere with the signal from the sample target. For Raman spectroscopy, topography is not an issue. Instead, it is mostly important to avoid interfering signals. To this effect it is best to leave the samples untreated, without any resins or coatings.

\subsubsection{Upper water column and cultured samples}

The extraction of diatom frustules from the water column can be done by filtering seawater and depositing the frustules on polycarbonate filters. During this $\mathrm{PhD}$. project, the filtration of water column samples was performed during expeditions in the Mediterranean (winter of 2016) and Red Sea (spring of 2017). By creating various size fractions, an effort was made to separate the diatom frustules from other particles. However, depending on the analytical technique of choice and goal of the analysis, additional steps were needed to isolate the frustules further and remove potential contamination. Three different approaches were tested:

1. Low-temperature ashing - At the Netherlands Institute for Sea Research (NIOZ), filters with diatom detritus were 'fired' with plasma in an attempt to remove organics and dirt. The filters were placed in both Petri dishes and small vials to experiment with different storing options. The samples spent around 6 hours in the 'oven'. The results were mixed as not all the filters were completely ashed, especially those placed in the vials. Also, in cases where the filter was successfully removed by ashing, some residual ash remained present and was not easily removed. However, at times where the filter was removed the frustules appeared to be well preserved and could be separated quite successfully from the container by rinsing or with the micromanipulator.

Pros: Removes unwanted organics; releases frustules from filter.

Cons: Ash-remains not easily removed; slow process.

2. Micromanipulator - Foraminifera are often picked by hand, using a fine brush, in order to isolate a single species for analysis. As diatom frustules are an order of magnitude smaller, this same setup is not possible. However, in the literature, an alternative has been presented for smaller organisms such as coccoliths and diatoms using a micromanipulator setup (Stoll and Shimizu, 2009; Snelling et al., 2013). During this PhD, the micromanipulator was used in combination with the previously mentioned ashing procedure. With the micromanipulator small movements can be made with a joystick, allowing for extreme precision $(<1 \mu \mathrm{m})$. Different tools can be fixed to the micromanipulator to fit the needs of the user. Initially, an attempt was made using an injector needle. This method uses drops of ethanol to improve the extraction of the particles. However, the size of the particles (diatoms, coccoliths ashes, dust) together with the unequal distribution of the particles in the sample complicated the targeting of individual diatom specimens. Instead, fine single brush hairs were attached. After considerable practice it was possible to pick individual frustules and deposit them in the preferred orientation. However, while it was possible to move the specimens within the sample, depositing them in a preferred orientation on a nanoSIMS holder was more difficult, as this moves away from the microscope. 
The slow but precise nature of this approach makes it predominantly suitable for studies interested in a specific species.

Pros: Individual frustules can be targeted; orientation of frustule can be altered.

Cons: Deposition on a nano-SIMS holder can lead to sample loss; Time-consuming.

3. Polycarbonate filter - The simplest way of measuring water column samples is directly measuring material on the polycarbonate filter as this requires very little preparation. However, this method does have a few caveats. Filtration does not always evenly distribute the diatom targets, potentially creating piles of frustules which are not suitable for nanoSIMS measurement. It should be noted that, as nanoSIMS analysis does not require a lot of sample, there are generally too many rather than too few individuals. Another potential caveat is the difficulty of performing cleaning procedures. Potentially, some simple cleaning steps can be performed with the original filtration setup, but for harsher cleaning steps the particles will have to be removed from the filter.

Pros: Quick and easy to use, relatively flat surface.

Cons: Limited cleaning possible; not always an even distribution.

\subsubsection{Sediment and sponge samples}

Sediment samples require very different sample preparation procedures. First of all, it is important to separate the frustules from the rest of the sediment. This includes, at minimum, sieving in order to isolate the size fraction with the frustules from other fossilized remains and the majority of clay minerals (10-75 $\mu \mathrm{m}$; Morley et al., 2004). After this additional mechanical and chemical cleaning steps can be performed to remove any remaining contamination (see Morley et al., 2004). After reaching the wanted levels of cleanliness, the next step is nanoSIMS preparation. For sediment frustules, the degree of fossilization should be kept in mind. As fossilization leads to sturdier frustules, the frustules should be broken before measurement if the aim is to target the frustule interior. The siliceous sponge spicules used in this thesis were not fossilized, but appear to have similar hardness to the fossil diatom frustules. So, for spicules, as with fossil frustules, it should be considered that it is not easy to burn through the entire structure with the typical primary ion currents used for nanoSIMS analysis (pA range). Considering these aspects of sediment and sponge samples, the following nanoSIMS preparation approaches were tested.

1. Pressing onto a carbon tape - This has been the method of choice in this thesis for the majority of the analyses looking at fossil diatom frustules. The prime benefits are the low cost and relative simplicity. This method consists of sticking a conductive carbon tape (PELCO Tabs ${ }^{\text {TM}}$; \#16084) onto an aluminum stub after which the diatom detritus is pressed onto the tape. This could be done by running a sterile needle through a sample, causing the frustules to stick to the needle, and rolling the needle onto the tape. The force at which this application occurs determines whether the frustules are kept intact or are broken. The combination of gold coating on top and the conductive carbon tape on the bottom ensures conductivity of the samples. Something to be considered with this sample preparation method is that strong organic signals can originate from the carbon tape. Therefore, this approach should be avoided when targeting organic signals (i.e. ${ }^{12} \mathrm{C},{ }^{12} \mathrm{C}^{14} \mathrm{~N}$ ).

Pros: Easy to use; cheap; possibility to break frustules.

Cons: Not useful when interested in organic signals; no control on orientation. 
2. Pressing onto an indium foil - When interested in the organic signals of frustules, a better approach is the use of a $99.99 \%$ indium foil (Alfa Aesar, $0.05 \mathrm{~mm}$ thick). The foil provides an ultra-clean background and by using a press, pressure can be dosed, and the grains can be securely located in the foil. For frustules it is not recommended to use a press, as this crushes the frustules to dust. Instead, we attached the foil to a stub with either glue or a carbon tape, and then pressed this stub onto the cleaned frustules manually. If the aim is to fracture the frustules, it is recommended to apply significant pressure, but otherwise a short press is sufficient. Determining suitable pressure is a matter of trial and error.

Pros: Ultra-clean background limits interfering signals, relatively simple method.

Cons: More expensive than carbon tape, difficult to create flat surface, no control on distribution.

3. Epoxy resin embedding - Embedding samples is a common practice for nanoSIMS measurements. This process can create a smooth cross-section ideal for measuring, for example, elemental ratio distribution within a foraminiferal shell (e.g. Geerken et al., 2019). Unfortunately, this technique is not compatible with diatom frustules. This is due to the smaller size and more porous structure of the frustules. However, in this thesis this approach has been applied with success to siliceous sponge spicules and quartz crystals. There are various ways to polish the embedded samples, these are discussed in detail in Chapter 6 of Roepert (2019). For the spicules we initially attempted a wet-polishing technique with diamond disks on turning tables, but this did not give sufficient control resulting in the complete abrasion of the spicule. Thus, we applied a manual approach for slower polishing. This approach included initial dry polishing with P4000 siliconcarbide grinding paper (Struers). This was followed by a fine polishing step using F1 diamond fluid on a polishing cloth. After final ethanol rinses and an ultrasonic bath, the resulting spicules proved sufficiently flat under the SEM and nanoSIMS.

Pros: Smooth surface; ability to create cross-sections.

Cons: Suitable only for larger physical structures, potential signals from resin and polishing suspensions.

\subsection{NanoSIMS data acquisition}

Most of the data in this thesis was acquired using the nanoSIMS 50L instrument at Utrecht University. Some background on how the nanoSIMS works is found in the introduction of this thesis and further information can be found in the literature (Roepert, 2019; Hoppe et al., 2013; Nuñez et al., 2018). In the following section, we will discuss specifically the application of the nanoSIMS technique to the measurement of biogenic silica.

\subsubsection{Elements and interferences}

With the nanoSIMS it is in principle possible to measure every element with the exception of noble gases. The ability to distinguish between all these different elements can be attributed to the high mass resolving power (MRP; defined as $\mathrm{M} / \Delta \mathrm{M}$ ) that can be achieved with the nanoSIMS instrument. Even in the case of not completely separated peaks, the high MRP (generally 35008500) of the nanoSIMS allows us to resolve most isobaric interferences. An overview of the different secondary ions targeted during this study and the possible interferences are presented below. For the studies presented in this thesis, the isobaric interferences did not pose a problem. 


\begin{tabular}{|c|c|c|c|}
\hline Secondary ion & $\mathrm{m} / \mathrm{z}$ & $\begin{array}{l}\text { Material to calibrate magnet } \\
\text { and detector positions* }\end{array}$ & Possible isobaric interferences in silica** \\
\hline${ }^{7} \mathrm{Li}^{+}$ & 7 & Spodumene (SPI) & - \\
\hline${ }^{11} B^{-}$ & 11 & Boron Nitride (SPI) & - \\
\hline${ }^{12} \mathrm{C}$ & 12 & Graphite & ${ }^{11} B^{1} H^{-}(701) ;{ }^{10} B^{1} H_{2}^{-}(420)$ \\
\hline${ }^{13} \mathrm{C}$ & 13 & Graphite & ${ }^{12} \mathrm{C}^{1} \mathrm{H}^{-}(2909) ;{ }^{11} \mathrm{~B}^{1} \mathrm{H}_{2}^{-}$ \\
\hline${ }^{16} \mathrm{O}^{-}$ & 16 & Graphite & - \\
\hline${ }^{18} \mathrm{O}^{-}$ & 18 & Graphite & ${ }^{1} \mathrm{H}_{2}{ }^{16} \mathrm{O}(1578) ;{ }^{17} \mathrm{O}^{1} \mathrm{H}^{-}(2309)$ \\
\hline${ }^{24} \mathrm{Mg}^{+}$ & 24 & Magnesium (SPI) & ${ }^{12} \mathrm{C}_{2}^{+}(1603) ;{ }^{23} \mathrm{Na}^{1} \mathrm{H}^{+}(1911)$ \\
\hline${ }^{12} \mathrm{C}^{14} \mathrm{~N}^{-}$ & 26 & Graphite & ${ }^{26} \mathrm{Mg}^{+}(1269)$ \\
\hline${ }^{12} \mathrm{C}^{15} \mathrm{~N}^{-}$ & 27 & Graphite & ${ }^{27} \mathrm{Al}^{+}(1454) ;{ }^{13} \mathrm{C}^{14} \mathrm{~N}^{-}(4271) ;{ }^{11} \mathrm{~B}^{16} \mathrm{O}^{-}(6566)$ \\
\hline${ }^{27} \mathrm{~A} l^{+}$ & 27 & Aluminum (SPI) & ${ }^{12} \mathrm{C}^{15} \mathrm{~N}^{-}(1453) ;{ }^{11} \mathrm{~B}^{16} \mathrm{O}^{-}(1190) ;{ }^{26} \mathrm{Mg}^{1} \mathrm{H}^{+}(3039)$ \\
\hline${ }^{28} \mathrm{Si}^{+}$ & 28 & Silicon (SPI) & ${ }^{27} \mathrm{Al}^{1} \mathrm{H}^{+}(2249) ;{ }^{12} \mathrm{C}^{16} \mathrm{O}^{+}(1555)$ \\
\hline${ }^{28} \mathrm{Si}^{-}$ & 28 & Silicon (SPI) & - \\
\hline${ }^{31} P^{-}$ & 31 & Indium phosphide (SPI) & - \\
\hline${ }^{32} \mathrm{~S}$ & 32 & Galena (SPI) & ${ }^{16} \mathrm{O}_{2}^{+}(1800)$ \\
\hline${ }^{40} \mathrm{Ca}^{-}$ & 40 & Calcite (SPI) & ${ }^{40} \mathrm{~K}^{+}(28383){ }^{16} \mathrm{O}^{12} \mathrm{C}_{2}^{+}(1236)$ \\
\hline${ }^{27} \mathrm{Al}^{16} \mathrm{O}^{-}$ & 43 & Aluminum (SPI) & ${ }^{32} S^{11} B^{-}(8737) ;{ }^{31} P^{12} C^{+}(15953)$ \\
\hline${ }^{56} \mathrm{Fe}^{+}$ & 56 & Iron (SPI) & ${ }^{40} \mathrm{Ca}^{16} \mathrm{O}^{+}(2479) ;{ }^{28} \mathrm{Si}_{2}(2957) ;{ }^{24} \mathrm{Mg}^{16} \mathrm{O}_{2}^{-}(1401)$ \\
\hline${ }^{28} \mathrm{Si}^{16} \mathrm{O}_{2}^{-}$ & 60 & Silicon (SPI) & ${ }^{32} \mathrm{~S}^{28} \mathrm{Si}(3377) ;{ }^{14} \mathrm{~N}_{2}{ }^{16} \mathrm{O}_{2}^{-}(2052)$ \\
\hline${ }^{64} \mathrm{Zn}^{+}$ & 64 & Zinc $(S P I)$ & ${ }^{48} \mathrm{Ti}^{16} \mathrm{O}^{-}(4661) ;{ }^{27} \mathrm{Al}^{37} \mathrm{Cl}^{+}(3439) ;{ }^{32} \mathrm{~S}_{2}(4263)$ \\
\hline${ }^{56} \mathrm{Fe}^{16} \mathrm{O}^{-}$ & 72 & Iron (SPI) & ${ }^{72} \mathrm{Ge}^{+}(9251) ;{ }^{28} \mathrm{Si}_{2}^{16} \mathrm{O}^{+}(3803)$ \\
\hline${ }^{138} \mathrm{Ba}^{+}$ & 138 & Barium fluoride (SPI) & ${ }^{137} \mathrm{Ba}^{1} \mathrm{H}^{+}(16408)$ \\
\hline
\end{tabular}

* SPI refers to the 02757-AB 59 Metals \& Minerals Standard (SPI supplies)

** The minimum MRP (M/ $\triangle M$, Cameca's $10-90 \%$ peak width definition) to be able to separate is given in parentheses; this list does not claim to be complete.

\subsubsection{Performance over time}

The strength of the nanoSIMS lies predominantly in the ability to image the distribution of different isotopes of a given element on a submicron scale. Quantification of the ratios between different elements is more challenging. One of the uncertainties that may possibly arise when measuring isotope or element ratios comes from the sensitivity of the detectors. Electron multiplier detectors, commonly used in this thesis, can become less sensitive over time as a result of ageing due to the incoming ion current. Furthermore, the degree of ageing can vary among detectors as they receive different ion flows (Gabitov et al., 2013). We have taken several measures to minimalize the effect this may have on the results.

1. Tuning of the pulse height distributions (PHD) of the detectors at the start of each analytical session. In this way, the starting conditions were comparable among sessions. Ageing during a session was assumed to be negligible as the incoming secondary ion currents were kept low during imaging (by reducing the primary ion current, when necessary).

2. Preferably detecting, in each session, the same masses on the same detectors.

3. Alternating between samples of different treatments within an experiment, and repeating measurements for a selected subset of samples during different analytical sessions.

With these precautions we are confident about the interpretation of relative variations in the ratios among samples. In contrast we are less confident about the interpretation of the absolute values, especially due to the lack of standards compatible with nanoSIMS analysis (see next). 


\subsubsection{Standards in nanoSIMS}

In order to quantify and compare ratios between measurements, methods and samples, a reliable standard is necessary. A suitable standard for nanoSIMS analysis needs to be spatially homogenous at the submicron scale to ensure measurement reproducibility. Preferably, the standard should have a similar matrix to the sample to avoid the influence of matrix-dependent differences in ionization efficiency and elemental fractionation (Dyar et al., 2001; Fayek, 2009; Shimizu, 2019). Roepert (2019) studied the possibility of creating a homogenous (carbonate) standard for nanoSIMS analysis, but this has proven difficult. For silica, standards with the necessary homogeneity for nanoSIMS analysis have also not yet been developed. Because of this, we attempted to calibrate our measurements in Chapter $2(\mathrm{Fe} / \mathrm{Si})$ by using three NIST (National Institute of Standards and Technology) glasses, which are standards with a $\mathrm{SiO}_{2}$ matrix that are used in a broad range of analytical methods. Our measurements showed that the range of $\mathrm{Fe} / \mathrm{Si}$ ion count ratios determined by nanoSIMS is not comparable to the certified error in the NIST standards (Fig. 6.1). On the one hand, nanoSIMS measurements run for sufficiently long periods of time showed very good precision $(0.7 \%$ Poisson error) and reproducibility when comparing adjacent measurement spots (15 x $15 \mu \mathrm{m}$ in size). On the other hand, a comparison of more distant measurement spots $>100$ $\mu \mathrm{m})$ showed pronounced variability (Fig. 6.1). Without sufficient homogeneity of standards, it is unrealistic to quantify element ratios to absolute values. Instead, we suggest that at present nanoSIMS studies can only tackle relative variability in element ratios within and between samples.

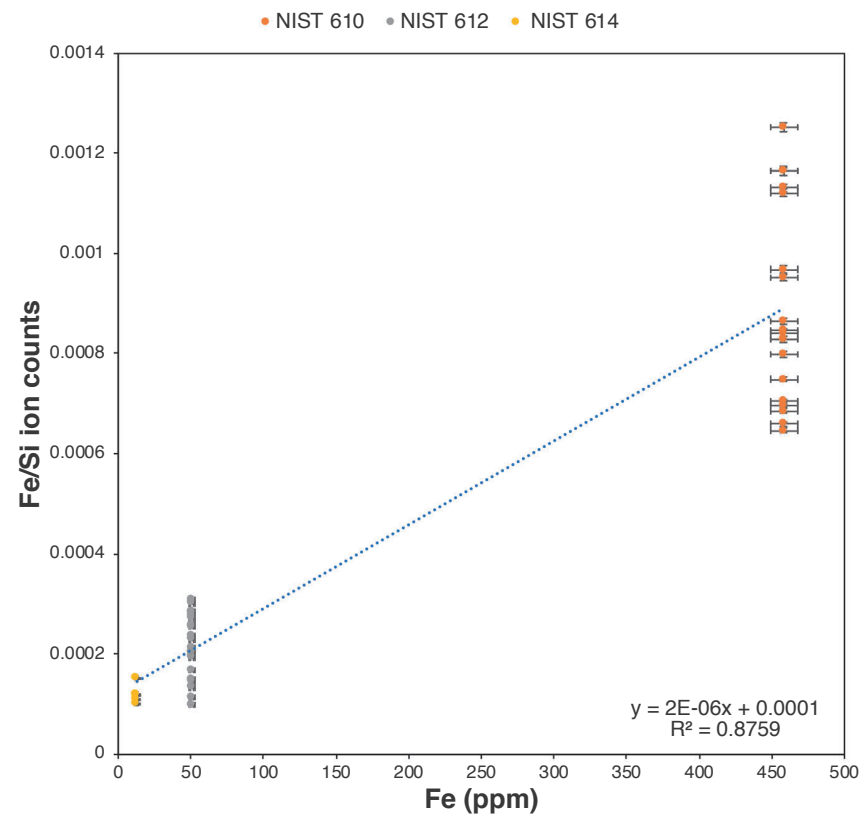

Figure 6.1: Fe content of certified NIST glass standards against Fe/Si ion count ratios determined by nanoSIMS. Horizontal error bars represent NIST certified error and the vertical error bars show nanoSIMS Poisson error, the latter determined by the amount of detected ions. 


\subsubsection{Primary ion implantation and pre-sputtering protocols}

During the course of this nanoSIMS research, one aspect has proven difficult to master: adequate primary ion implantation and pre-sputtering. When the sample is bombarded by primary ions, the primary ions become "implanted" into the sample matrix which leads to an increase in the secondary ion yields. This can be done by using either low energy primary ions (the so-called lowenergy implantation procedure) or high energy primary ions (the so-called pre-sputtering). While the ion yields can be (approximately) monitored during the pre-sputtering procedure, this is not possible during the low-energy implantation procedure as it does not lead to the erosion of the sample material. Either way, primary ion implantation and pre-sputtering are essential components of a nanoSIMS measurement that determine the secondary ion yields and thus have the potential to strongly influence the final element-to-element ratios. Achieving stable secondary ion yields is, however, not as straightforward as it might seem, as each measurement has different needs. This depends on the material, ion of interest, incoming current, measuring time, etc. During this research, we targeted different types of material, and below we discuss the successful protocols that we applied consistently for the given type of material.

\section{Cultured diatom frustules}

Of all the nanoSIMS targets studied during this research, frustules of cultured diatom have proven to be most fragile and challenging to analyse. Due to this, very conservative protocols were applied to preserve frustule integrity. Specifically, we used the low-energy primary ion implantation technique to increase the secondary ion yields and thus improve the measurement precision (i.e., minimize the Poisson error). An additional precaution was taken by performing the instrument tuning on a nearby frustule. This was decided as the tuning procedure often resulted in strongly altered frustules. With this approach it was possible to preserve the target frustule for the measurement while obtaining sufficient ion counts in each pixel of the imaged area.

However, during data processing, considerable attention had to be paid to the depth variation of the ion count ratios. This variation was observed during both the initial and final stages of the measurement (Fig. 6.2). For isotope ratios such as ${ }^{18} \mathrm{O} /{ }^{16} \mathrm{O}$ the initial variation (first $~ 50$ planes) likely represented the actual variation within the sample. However, for element ratios such as $\mathrm{Al} / \mathrm{Si}$ the initial variation was more likely associated with the unequal rates at which secondary ion yields for different elements stabilize during the LE-implantation procedure. The variation during the final stages of the measurement was largely due to uneven erosion of the sample material, whereby signals in some areas still originated from the frustule while signals in other areas originated from the underlying substrate (Fig. 6.2). Together, these observations highlight the need to carefully select the pixels and planes over which the ion counts are accumulated when obtaining the final frustulespecific ion count ratio. Our approach for frustules targeted with the LE-implantation therefore included the removal of the initial unstable planes when these were judged to be an artifact (dotted lines in Fig. 6.2d-f). For this determination, the coefficient of variance due to depth, and not the Poisson error derived from total accumulated ion counts, served as a measure of the precision of the $\mathrm{El} / \mathrm{Si}$ ratio.

\section{Fossil diatom frustules}

Compared to the cultured diatom frustules, the fossilized frustules were significantly more robust. This means that the pre-sputtering protocol with high-energy primary Cs+ ions could be used to obtain stable ion yields earlier while maintaining sufficient material for measurement. Initially, we used commonly applied pre-sputtering settings (D1-1; $\left.\mathrm{FC}_{\mathrm{O}}=20 \mathrm{pA}\right)$ with the assumption that 

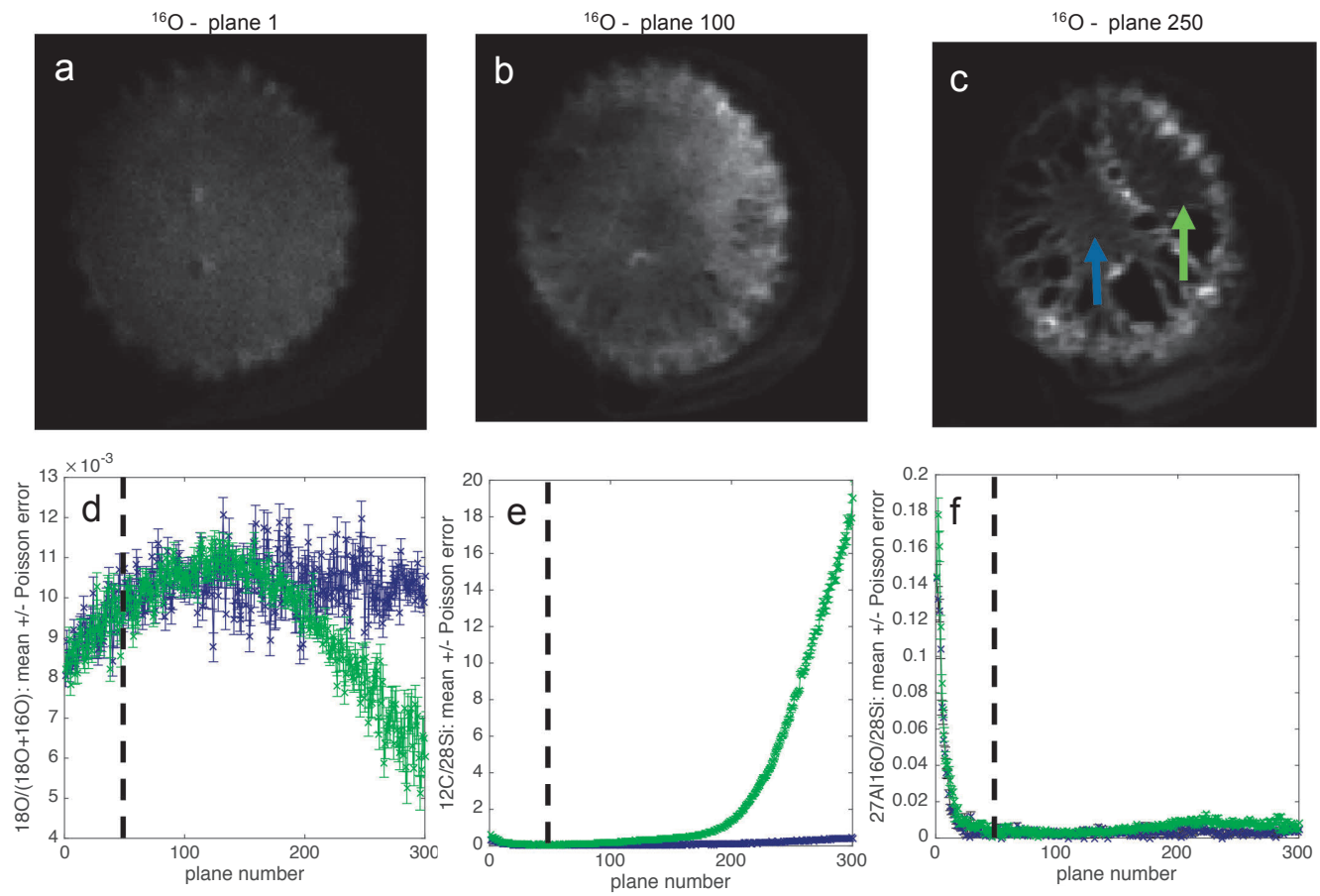

Figure 6.2: NanoSIMS imaging of $\mathbf{1 8 O} / \mathbf{1 6 O}$ in a diatom frustule. (a-c) Variation in the ${ }^{16} \mathrm{O}$ ion counts with the measurement plane. (a) Initially, ion counts are low due to insufficient implantation of the primary $\mathrm{Cs}^{+}$ions into the frustule matrix by the low-energy implantation procedure performed before the measurement. (b) As more highenergy $\mathrm{Cs}^{+}$ions are implanted into the matrix during the measurement, the secondary ion yield increases leading to higher ion counts. (c) However, sputtering of the frustule material is uneven, leading to increasing heterogeneity of the ion count image. Dark pixels correspond to areas where the frustule was completely sputtered away. (d-f) Ratios as a function of the measurement plane (i.e., depth), shown for two areas on the frustule. Dotted line marks potential start of the accumulation if unstable planes are removed (Green and blue data points correspond to areas pointed to by the green and blue arrow in panel b). (d) ${ }^{18} \mathrm{O}$ atom fraction; Following an initial increase (planes 0-50), the ${ }^{18} \mathrm{O} /{ }^{16} \mathrm{O}$ ratio stabilizes for the blue profile (planes 50-300), but the red profile starts to decrease towards the value corresponding to the underlying carbon-tape substrate as the frustule matrix becomes sputtered away (planes $150-300) \cdot(\mathrm{e}){ }^{12} \mathrm{C} /{ }^{28} \mathrm{Si}$; The green depth profile increases greatly as the underlying substrate becomes visible (planes $150-300)$. (f) ${ }^{27} \mathrm{Al} /{ }^{28} \mathrm{Si}$; The profile is dominated by a rapid decrease in the first 50 planes. This is attributed to the LE-implantation approach which led to initial unequal sputtering rates. 
this pre-sputtering, followed by tuning, would eventually result in stable secondary ion yields and thus no variation of the secondary ion count ratios with depth. However, preliminary data analysis showed that this was not sufficient. Therefore, the final protocol that we used consistently for all measurements of fossilized frustules included the following steps. First, we applied D1-0 with $\mathrm{FC}_{\mathrm{O}}$ $=20$ until detector \#3 (tuned to 28Si) reached the count rate of $170 \mathrm{kcps}$. By setting C4y to 50 bits, which deflects large part of the secondary ions away from the detector, we were able to heavily bombard the area of interest while monitoring the secondary ion yield without overloading the detector. Once the $170 \mathrm{kcps}$ count rate was reached, we switched to D1-1 for a more focused beam, and continued pre-sputtering until the 28Si counts stabilized. During data processing we analyzed the variation of the El/Si ion count ratio with depth, and deemed the measurement acceptable if, and only if, the coefficient of variation with depth was below a pre-determined threshold. We decided on the threshold of $20 \%$ based on a thorough analysis of all measured frustules.

\section{Sponge spicules}

The sponge spicules appeared to be even more robust than the fossil diatom frustules. Therefore, the same rigorous pre-sputtering protocol could be applied. However, a difference arose in the susceptibility to charging. For the spicules, the electron gun (e-gun) was an absolute must; without it, charging became an issue already during pre-sputtering. The electrons from the e-gun helped compensate for the positive charge buildup from the implanted primary ions.

\subsection{NanoSIMS data processing}

There are several software packages suitable for the processing of nanoSIMS data including the Cameca software WinImage, the program L'Image developed by L. Nittler, the ImageJ plugin OpenMIMS, or the Matlab-based freeware Look@nanoSIMS. Because in this thesis we needed to quickly develop and apply new image processing routines, we decided to use the updated version of the program Look@NanoSIMS (Polerecky et al., 2012). In the next section we describe some points to consider when dealing with diatom frustules during data-processing.

\subsubsection{Avoiding edge effects}

The strength of nanoSIMS lies in its high-resolution imaging capabilities. This allowed us to target specific areas using the Region of Interest (ROI) tool. As mentioned earlier in this chapter, sample topography can form a problem for the interpretation of nanoSIMS data. For centric diatom frustules, much of the topography comes from the edges of the frustule, in particular when targeting the interior of the frustule. By applying the ROI tool, we could target areas away from the edges during the data processing step (Fig. 6.3a-b). At times, we attempted to choose a field of view in the center of the frustule already during the measurement, to keep the edges out of view. However, this often resulted in greater charging effects (Fig. 6.3c) and was therefore rarely pursued. 

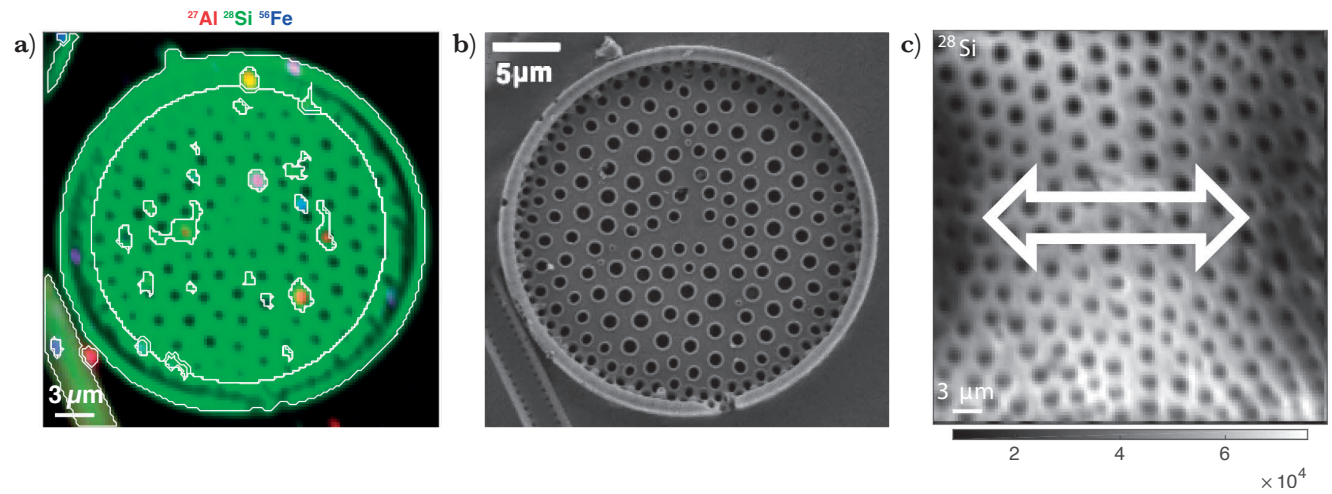

Figure 6.3: Imaging of centric diatom frustules by NanoSIMS. (a-b) RGB overlay of the secondary ion count images $\mathrm{Al}+, \mathrm{Si}+$ and $\mathrm{Fe}+$ measured from the interior of a diatom frustule. White lines represent ROI outlines, panel (b) shows the corresponding SEM image. (c) Image of the secondary ions 28Si- measured from an exterior of another frustule of a centric diatom. If the charge deposited onto the sample surface during the measurement is not removed or compensated, e.g., due to insufficient conductivity of the sample or ineffective application of the e-gun, primary ions can no longer "illuminate" the sample surface homogeneously. This leads to 'shadows' in the images of the secondary ions, as marked by the arrow.

\subsubsection{The influence of pores}

Another feature specific for diatom frustules that needs to be considered during data analysis is the presence of pores. The impact that pores can have on the accumulated $\mathrm{El} / \mathrm{Si}$ ion count ratio of the frustule ROI depends on several factors, including the relative size of the frustule ROI covered by the pores, and the type of substrate. Potentially, the substrate material may produce signals that can interfere with the signals from the frustule. For example, when studying an ${ }^{18} \mathrm{O}$-enriched fossil frustule, the natural abundance ${ }^{18} \mathrm{O}$ signals originating from the underlying filter are clearly seen through the pores in the ion count ratio image (Fig. 6.4a-c). The impact on the final ${ }^{18} \mathrm{O}$ atom fraction in this example is limited, but this might increase as the difference in ${ }^{18} \mathrm{O}$ atom fraction between the substrate and the silica increases. When studying $\mathrm{Fe} / \mathrm{Si}$ ratios, ion counts for both elements were low in the pores. Thus, exclusion of pores from the final analysis did not lead to significant differences between the frustule-specific $\mathrm{Fe} / \mathrm{Si}$ ratio, provided that the areas corresponding to Fe-rich hot-spots were already excluded (Fig. 6.4d-f). 
a)

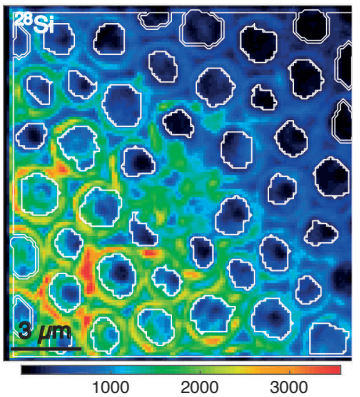

d)

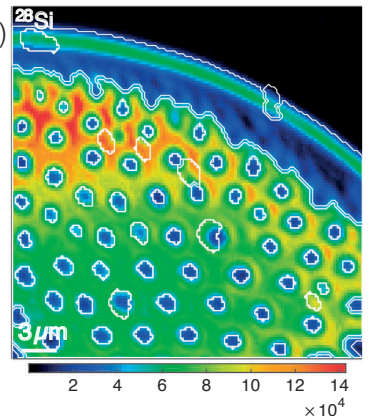

b)
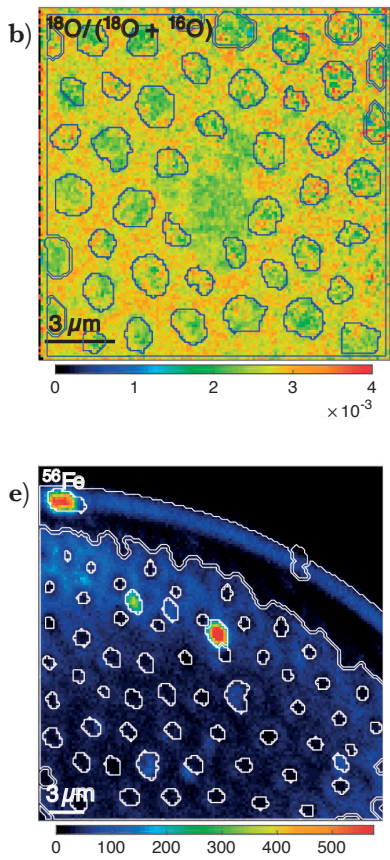
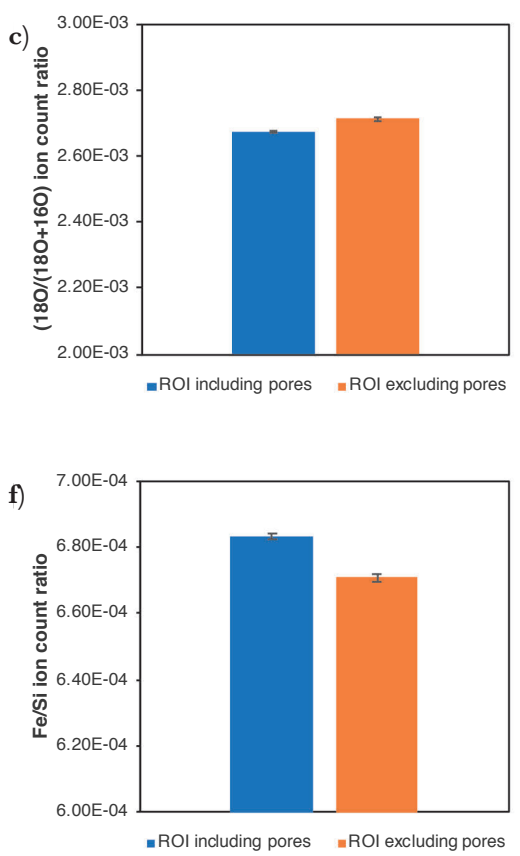

Figure 6.4: Illustrating the influence of the pores on ion count ratio. (a-c) Influence pores on the ${ }^{18} \mathrm{O}$ atom ratio (a) Accumulated secondary ion count image of ${ }^{28} \mathrm{Si}$ with white lines indicating ROI definition. (b) Accumulated secondary ion count ratio image of the ${ }^{18} \mathrm{O}$ atom fraction with blue lines indicating ROI definition. Large green area in center represents an $\mathrm{Al}$ contamination, not a pore (c) Bar chart illustrating the influence of removing the pores from the final ROI on the ${ }^{18} \mathrm{O}$ atom ratio. Error bars indicate Poisson error $(0.15 \%)$. An increase does occur $(1.84 \%)$, greater than the Poisson error, but significantly smaller than the variability between samples (d-f) Influence pores on the $\mathrm{Fe} / \mathrm{Si}$ ion count ratio (d-e) Accumulated secondary ion count image with white lines indicating ROI definition. d) $28 \mathrm{Si}$ e) ${ }^{56} \mathrm{Fe}$; (f) Bar chart illustrating the influence of removing the pores from the final ROI on the ${ }^{56} \mathrm{Fe} /{ }^{28} \mathrm{Si}$ ion count ratio. Error bars indicate Poisson error $(0.15 \%)$. A reduction does occur $(1.84 \%)$, greater than the Poisson error, but significantly smaller than the variability between samples. 


\subsection{Which other methods are suitable for the analysis of diatom frustules and other forms of biogenic silica?}

In addition to NanoSIMS, which was the main method applied in this thesis, we also used several other analytical methods to study biogenic silica. The experiences with each method are evaluated below.

\subsubsection{Secondary lon Mass Spectrometry (SIMS)}

The SIMS technique utilizes the same basic principles as the nanoSIMS but differs in the achievable spatial resolution (not better than $\sim 500 \mathrm{~nm}$ ), the primary ion currents used (nA), and the relationship between transmission and mass resolution power. Due to this SIMS does not allow imaging of diatom frustules in the way nanoSIMS does. However, this technique has proven to be a valuable partner to the nanoSIMS in Chapter 2 where we measured $\mathrm{Fe} / \mathrm{Si}$ values in fossil diatom frustules. While the nanoSIMS provided detailed spatial information on the frustules, the conventional SIMS method allowed more robust quantification of the differences between samples. This was predominantly due to the high currents employed by SIMS (typically in the nA range, compared to the pA range typically employed in nanoSIMS), which increased the overall ion counts and hence also the measurement throughput. The SIMS can perform spot analyses for determining $\mathrm{El} / \mathrm{Si}$ ratios of (large) individual frustules or groups of fossils, but without imaging capabilities. The problem of insufficiently homogeneous standards, as discussed in section 6.3.3, is less important for SIMS because higher volumes are sputtered, smoothing out the heterogeneity of the standard.

\subsubsection{Raman micro-spectroscopy}

Raman micro-spectroscopy has proven to be an important complementary method to NanoSIMS in two chapters of this thesis. In Chapter 3, we used it to confirm the spatial patterns observed in the nanoSIMS data. With the nanoSIMS we were able to image organics in frustules with a submicron resolution, but our confidence in the nanoSIMS data interpretation was lower due to the effects of surface topography on the secondary ion yield ("edge effects"). Raman microspectroscopy is also a surface technique, but it is rather insensitive to surface topography as it integrates the signal over a relatively large vertical interval (down to $\sim 1 \mu \mathrm{m}$ ). However, a downside of the Raman technique, compared to the nanoSIMS, is the lower lateral resolution ( $\mu \mathrm{m}$ scale). Overall, our Raman micro-spectroscopy measurements confirmed the large-scale spatial patterns observed in the nanoSIMS data, but not the finer details. On its own, Raman micro-spectroscopy is of limited value for the analysis of submicron variability in diatom frustules, but together with the nanoSIMS it provides a powerful verification method.

In Chapter 4, we used Raman micro-spectroscopy to assess in more detail the chemical structures (e.g., bonds) in which the target element - oxygen - participated within the different silica targets. This information is inaccessible to nanoSIMS, which only provides information on the distribution of the target element itself. In this way, we were able to identify the different O-pools present in the silica of ${ }^{18} \mathrm{O}$-enriched sponge spicules and diatom frustules, in an attempt to discern where the nanoSIMS signals originated from. 


\subsubsection{Electron Probe Microanalyser}

The latest state-of-the-art JEOL JXA-8530F Hyperprobe Field Emission Electron probe microanalyser (EPMA) was tested as a 'scouting' technique in support of the nanoSIMS, primarily to create a 'snapshot' of the different trace elements present in the diatom frustules. This seemed a suitable technique as it is quicker, simpler and cheaper than the nanoSIMS, but still has a sufficient spatial resolution to target individual specimens. If this were successful, it could make nanoSIMS measurements more efficient by providing the relative abundance of elements to be targeted. Unfortunately, the initial experiments done in November 2016 showed that the EPMA was not effective for the analysis of diatom frustules. The small thickness of the valves $(\sim 200 \mathrm{~nm})$ was limiting the acquisition of data that could be reliably interpreted. Therefore, the use of EPMA was not pursued any further.

\subsubsection{Transmission electron microscope (TEM)}

In November 2018, measurements were performed using the FEI Titan-TEM facilities at the King Abdullah University of Science and Technology. As this technique has an even higher spatial resolution (down to $0.2 \mathrm{~nm}$ ) than the nanoSIMS, it was aimed to image the distribution of organic signals around the pores as observed earlier with the nanoSIMS (in Chapter 3). While it was possible to get high-resolution images of the pores, we were unable to extract any organic signals. Apparently, the relatively low abundance of organics in diatom frustules was beyond the sensitivity of Titan-TEM.

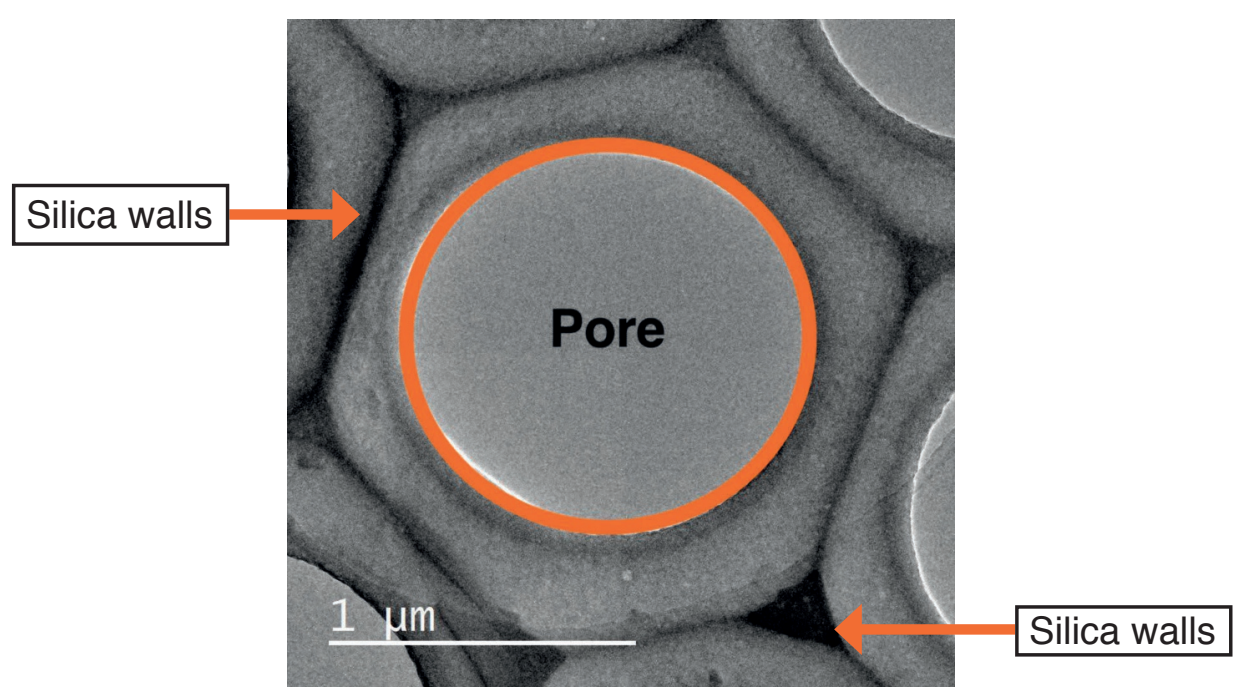

Figure 6.5: TEM imaging of fossil centric diatom frustule pores showing the silica structure. TEM image looking down on a pore in the frustule. Dark areas indicate areas of silica thickening. The silica walls are particularly evident. 


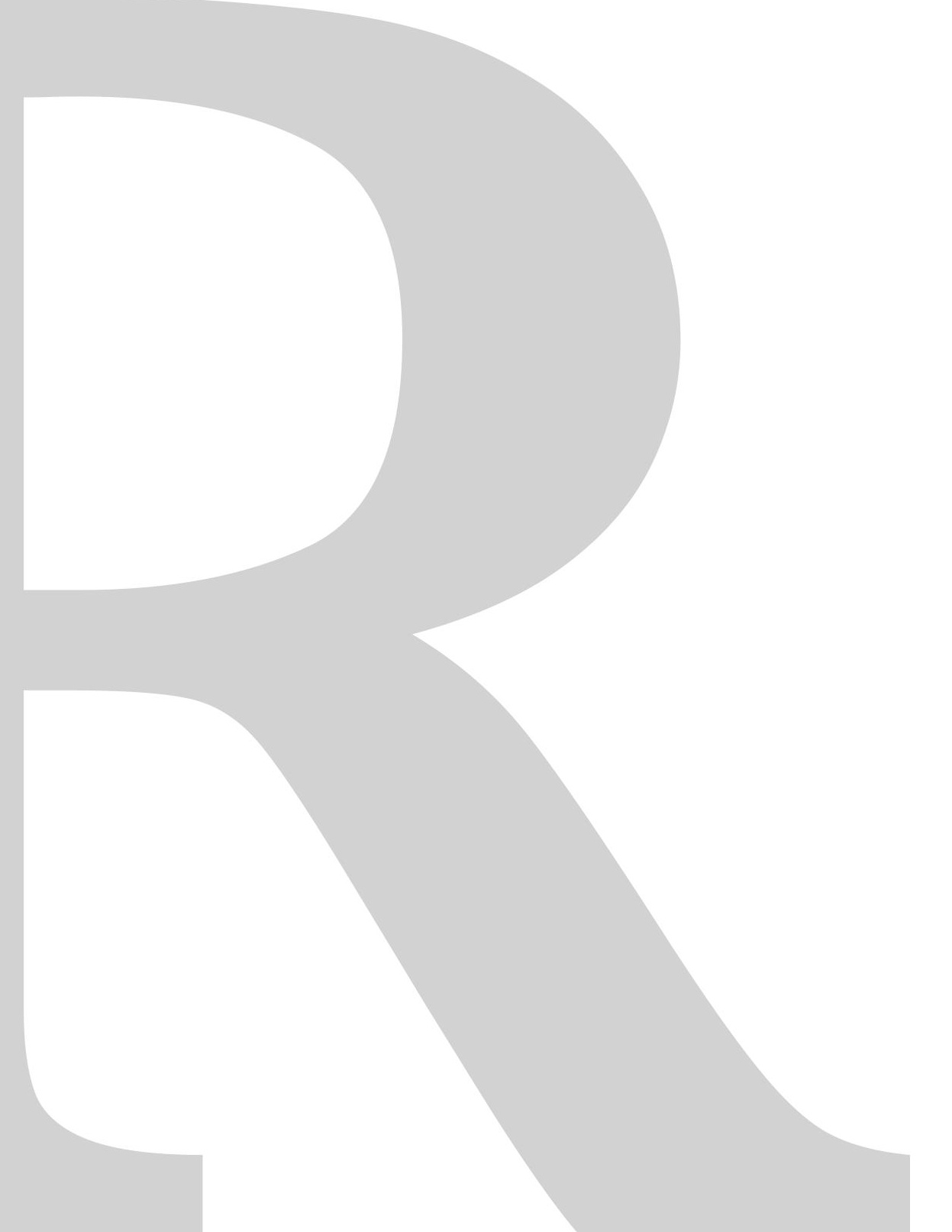




\section{References}

\section{Chapter 1}

Andersen M. B., Vance D., Archer C., Anderson R. F., Ellwood M. F. and Allen C. S. (2011) The Zn abundance and isotopic composition of diatom frustules, a proxy for Zn availability in ocean surface seawater. Earth Planet. Sci. Lett. 301, 137-145. Available at: http://dx.doi.org/10.1016/j.epsl.2010.10.032.

Arellano-Torres E., Pichevin L. E. and Ganeshram R. S. (2011) High-resolution opal records from the eastern tropical Pacific provide evidence for silicic acid leakage from HNLC regions during glacial periods. Quat. Sci. Rev. 30, 1112-1121. Available at: http:// dx.doi.org/10.1016/j.quascirev.2011.02.002.

Armbrust E. V. (2009) The life of diatoms in the world's oceans. Nature 459, 185-192. Available at: http://wrew.nature.com/ doifinder/10.1038/nature08057.

Azam F., Hemmingsen B. B. and Volcani B. E. (1973) Germanium incorporation into the silica of diatom cell walls. Arch. Mikrobiol. 92, 11-20.

Bareille G., Labracherie M., Mortlock R. A., Maier-Reimer E. and Froelich P. N. (1998) A test of (Ge/Si)opalas a paleorecorder of (Ge/Si)seawater. Geology 26, 179-182.

Basile-Doelsch I., Meunier F. D. and Parron C. (2005) Another continental pool in the terrestrial silicon cycle. Nature 433, 399-402. Available at: http://wrere.ncbi.nlm.nih.gov/pubmed/15674287.

van Bennekom A. F., Fred Jansen F. H., van der Gaast S. F., van Iperen 7. M. and Pieters F. (1989) Aluminium-rich opal: an intermediate in the preservation of biogenic silica in the Zaire (Congo) deep-sea fan. Deep Sea Res. Part A, Oceanogr. Res. Pap. 36, 173-190.

Van Beusekom 7.E.E., 1989. Wechselwirkungen zwischen gelösten Aluminium und Phytoplankton in marinen Gewässern. PhD thesis, Hamburg University, 164pp.

Brandriss M. E., O'Neil F. R., Edlund M. B. and Stoermer E. F. (1998) Oxygen isotope fractionation between diatomaceous silica and water. Geochim. Cosmochim. Acta 62, 1119-1125.

Brewer T. S., Leng M. F., Mackay A. W., Lamb A. L., Tyler F. F. and Marsh N. G. (2008) Unravelling contamination signals in biogenic silica oxygen isotope composition: the role of major and trace element geochemistry. F. Quat. Sci. 23, 321-330.

Bridoux M. C. and Ingalls A. E. (2010) Structural identification of long-chain polyamines associated with diatom biosilica in a Southern Ocean sediment core. Geochim. Cosmochim. Acta 74, 4044-4057. Available at: http://dx.doi.org/10.1016/j.gca.2010.04.010.

Bruland K. W., Knauer G. A. and Martin F. H. (1978) Zinc in north-east Pacific water. Nature 271, 741-743.

Brümmer, F. (2003). "Living inside a glass box-silica in diatoms," in Progress in Molecular and Subcellular Biology, ed W. E. G. Müller (Berlin; Heidelberg: Springer-Verlag), 3-9.

Brzezinski M. A., Pride C. F., Franck V. M., Sigman D. M., Gruber N., Rau G. H. and Coale K. H. (2002) Brzezinski et al 2002. pdf. 29, 3-6.

Cappellen P. Van and Qiu L. (1997) Biogenic silica dissolution in sediments of the Southern Ocean: II. Kinetics. Deep. Res. Part II Top. Stud. Oceanogr. 44, 1129-1149.

Cha, 7. N. N., Shimizu, K., Zhou, Y., Christiansen, S. C. C., Chmelka, B. F. F., Stucky, G. D. D., et al. (1999). Silicatein filaments and subunits from a marine sponge direct the polymerization of silica and silicones in vitro. Proc. Natl. Acad. Sci. U.S.A. 96, 361-365, doi: $10.1073 /$ pnas.96.2.361

Chang A. S., Pichevin L., Pedersen T. F., Gray V. and Ganeshram R. (2015) New insights into productivity and redox-controlled trace element ( $\mathrm{Ag}, \mathrm{Cd}, \mathrm{Re}$, and Mo) accumulation in a $55 \mathrm{kyr}$ long sediment record from Guaymas Basin, Gulf of California. Paleoceanography 30, 77-94. 
Chapligin B., Leng M. F., Webb E., Alexandre A., Dodd F. P., Ijiri A., Lücke A., Shemesh A., Abelmann A., Herzschuh U., Longstaffe F. F., Meyer H., Moschen R., Okazaki 1., Rees N. H., Sharp Z. D., Sloane H. F., Sonzogni C., Swann G. E. A., Sylvestre F., Tyler 7. F. and Yam R. (2011) Inter-laboratory comparison of oxygen isotope compositions from biogenic silica. Geochim. Cosmochim. Acta 75, $7242-7256$.

Des Combes H. F., Esper O., De La Rocha C. L., Abelmann A., Gersonde R., Yam R. and Shemesh A. (2008) Diatom $813 C, 815 \mathcal{N}$, and $C / N$ since the last glacial maximum in the southern ocean: potential impact of species composition. Paleoceanography 23, 1-12.

Conley D. F. (2002) Terrestrial ecosystems and the global biogeochemical silica cycle. Global Biogeochem. Cycles 16, 68-1-68-8.

Conley D. F., Frings P. F., Fontorbe G., Clymans W., Stadmark F., Hendry K. R., Marron A. O. and De La Rocha C. L. (2017) Biosilicification drives a decline of dissolved si in the oceans through geologic time. Front. Mar. Sci. 4.

Crosta X., Sturm A., Armand L. and Pichon F. F. (2004) Late Quaternary sea ice history in the Indian sector of the Southern Ocean as recorded by diatom assemblages. Mar. Micropaleontol. 50, 209-223.

Damsté F. S. S., Muyzer G., Abbas B., Rampen S. W., Massé G., Allard W. G., Belt S. T., Robert F. M., Rowland S. F., Moldowan 7. M., Barbanti S. M., Fago F. F., Denisevich P., Dahl F., Trindade L. A. F. and Schouten S. (2004) The Rise of the Rhizosolenid Diatoms. Science (80-.). 304, 584-587.

Demarest M. S., Brzezinski M. A. and Beucher C. P. (2009) Fractionation of silicon isotopes during biogenic silica dissolution. Geochim. Cosmochim. Acta 73, 5572-5583. Available at: http://linkinghub.elsevier.com/retrieve/pii/S0016703709004177.

Dixit S., Van Cappellen P. and Van Bennekom A. F. (2001) Processes controlling solubility of biogenic silica and pore water build-up of silicic acid in marine sediments. Mar. Chem. 73, 333-352.

Dodd F. P. and Sharp Z. D. (2010) A laser fluorination method for oxygen isotope analysis of biogenic silica and a new oxygen isotope calibration of modern diatoms in freshwater environments. Geochim. Cosmochim. Acta 74, 1381-1390. Available at: http://dx.doi. org/10.1016/j.gca.2009.11.023.

Dodd F. P., Wiedenheft W. and Schwartz F. M. (2017) Dehydroxylation and diagenetic variations in diatom oxygen isotope values. Geochim. Cosmochim. Acta 199, 185-195. Available at: http://dx.doi.org/10.1016/j.gca.2016.11.034.

Egon T. Degens (2), Samuel Epstein (1962) Relationship Between 018/016 Ratios in Coexisting Carbonates, Cherts, and Diatomites: GEOLOGICAL NOTES. Am. Assoc. Pet. Geol. Bull. 46, 534-542.

Ehlert C., Doering K., Wallmann K., Scholz F., Sommer S., Grasse P., Geilert S. and Frank M. (2016) Stable silicon isotope signatures of marine pore waters - Biogenic opal dissolution versus authigenic clay mineral formation. Geochim. Cosmochim. Acta 191, $102-$ 117. Available at: http://dx.doi.org/10.1016/j.gca.2016.07.022.

Ellwood M. F. and Hunter K. A. (2000) The incorporation of zinc and iron into the frustule of the marine diatom Thalassiosira pseudonana. Limnol. Oceanogr. 45, 1517-1524.

Frings P.F., Clymans W., Fontorbe G., De La Rocha C. L. and Conley D. F. (2016) The continental Si cycle and its impact on the ocean Si isotope budget. Chem. Geol. 425, 12-36. Available at: http://dx.doi.org/10.1016/j.chemgeo.2016.01.020.

Froelich, P. N., Hambrick, G. A., Andreae, M. O., Mortlock, R. A., and Edmond, F. M., 1985, The geochemistry of inorganic germanium in natural waters: Journal of Geophysical Research, v. 90, p. 1133-1141.

Froelich P. N., Blanc V., Mortlock R. A., Chillrud S. N., Dunstan W., Udomkit A. and Peng T. -H (1992) River Fluxes of Dissolved Silica to the Ocean Were Higher during Glacials: Ge/Si In Diatoms, Rivers, and Oceans. Paleoceanography 7, 739-767.

Gehlen M., Beck L., Calas G., Flank A. M., Van Bennekom A. F. and Van Beusekom 7. E. E. (2002) Unraveling the atomic structure of biogenic silica: Evidence of the structural association of Al and Si in diatom frustules. Geochim. Cosmochim. Acta 66, 1601-1609.

Hendry K. R., Georg R. B., Rickaby R. E. M., Robinson L. F. and Halliday A. N. (2010) Deep ocean nutrients during the Last Glacial Maximum deduced from sponge silicon isotopic compositions. Earth Planet. Sci. Lett. 292, 290-300. Available at: http://dx.doi. org/10.1016/j.epsl.2010.02.005.

Hendry K. R., Leng M. F., Robinson L. F., Sloane H. J., Blusztjan F., Rickaby R. E. M., Georg R. B. and Halliday A. N. (2011) Silicon isotopes in Antarctic sponges: An interlaboratory comparison. Antarct. Sci. 23, 34-42.

Hendry K. R. and Rickaby R. E. M. (2008) Opal (Zn/Si) ratios as a nearshore geochemical proxy in coastal Antarctica. Paleoceanography 23, 1-12. 
Hendry K. R. and Robinson L. F. (2012) The relationship between silicon isotope fractionation in sponges and silicic acid concentration: Modern and core-top studies of biogenic opal. Geochim. Cosmochim. Acta 81, 1-12. Available at: http://dx.doi.org/10.1016/j. gca.2011.12.010.

Hildebrand M., Lerch S. F. L. and Shrestha R. P. (2018) Understanding diatom cell wall silicification-moving forward. Front. Mar. Sci. 5, 1-19.

Hutchins D. A. and Bruland K. W. (1998) Iron-limited growth and Si:N ratios in a costal upwelling regime. Nature 393, 561-564.

Iler R. K. (1973) Effect of adsorbed alumina on the solubility of amorphous silica in water. F. Colloid Interface Sci. 43, 399-408.

Ingall E. D., Diaz 7. M., Longo A. F., Oakes M., Finney L., Vogt S., Lai B., Tager P. L., Twining B. S. and Brandes F. A. (2013) Role of biogenic silica in the removal of iron from the Antarctic seas. Nat. Commun. 4, 1981. Available at: http://wwrenature. com/doifinder/10.1038/ncomms2981.

Ingalls A. E., Lee C., Wakeham S. G. and Hedges F. I. (2003) The role of biominerals in the sinking flux and preservation of amino acids in the Southern Ocean along $170^{\circ} \mathrm{W}$. Deep. Res. Part II Top. Stud. Oceanogr. 50, 713-738.

Kammer M., Hedrich R., Ehrlich H., Popp F., Brunner E. and Krafft C. (2010) Spatially resolved determination of the structure and composition of diatom cell walls by Raman and FTIR imaging. Anal. Bioanal. Chem. 398, 509-517.

Karsh, K.L., Trull, T.W., Lourey, M.F. and Sigman, D.M. (2003). Relationship of nitrogen isotope fractionation to phytoplankton size and iron availability during the Southern Ocean Iron RElease Experiment (SOIREE). Limnology and Oceanography, 48, 1058 1068.

Kilburn, M. R. and Wacey, D. (2014). Nanoscale secondary ion mass spectrometry (NanoSIMS) as an analytical tool in the geosciences. In Grice, K., editor, Principles and Practice of Analytical Techniques in Geosciences, pages 1-34. The Royal Society of Chemistry Cambridge.

Koning, E., Epping, E., van Raaphorst, W., 2002. Determinig biogenic silica in marine samples by tracking silicate and aluminum concentrations in alkaline leaching solutions. Aquat. Geochem. 8, 39-67.

Koning E., Gehlen M., Flank A. M., Calas G. and Epping E. (2007) Rapid post-mortem incorporation of aluminum in diatom frustules: Evidence from chemical and structural analyses. Mar. Chem. 103, 97-111.

Kröger $\mathcal{N}$. and Poulsen N. (2008) Diatoms - From Cell Wall Biogenesis to Nanotechnology. Annu. Rev. Genet. 42, 83-107. Available at: http://wrww.annualreviews.org/doi/10.1146/annurev.genet.41.110306.130109.

De la Rocha C. L. (2003) Silicon isotope fractionation by marine sponges and the reconstruction of the silicon isotope composition of ancient deep water. Geology 31, 423-426.

De La Rocha C. L. (2006) Opal-based isotopic proxies of paleoenvironmental conditions. Global Biogeochem. Cycles 20, 1-11.

De La Rocha C. L., Brzezinski M. A. and DeNiro M. J. (1997) Fractionation of silicon isotopes by marine diatoms during biogenic silica formation. Geochim. Cosmochim. Acta 61, 5051-5056.

Leclerc A. F. and Labeyrie L. (1987) Temperature dependence of the oxygen isotopic fractionation between diatom silica and water. Earth Planet. Sci. Lett. 84, 69-74.

Leng M. F., Swann G. E. A., Hodson M. F., Tyler F. F., Patwardhan S. V. and Sloane H. F. (2009) The potential use of silicon isotope composition of biogenic silica as a proxy for environmental change. Silicon 1, 65-77.

Leng M. F., Swann G. E. A. and Leng M. F. (2010) Stable isotopes from diatom silica. Diatoms Appl. Environ. Earth Sci. Second Ed., 575-589.

Maldonado M., López-Acosta M., Sitjà C., García-Puig M., Galobart C., Ercilla G. and Leynaert A. (2019) Sponge skeletons as an important sink of silicon in the global oceans. Nat. Geosci.

Mann, S., and Perry, C. C. (1986). "Structural aspects of biogenic silica," in Silicon Biochemistry, CIBA Foundation Symposium 121, eds D. Evered and M. O'Connor (New Tork, NY: Wiley), 40-58.

Matheney R. K. and Knauth L. P. (1989) Oxygen-isotope fractionation between marine biogenic silica and seawater. Geochim. Cosmochim. Acta 53, 3207-3214.

Matsumoto K., Sarmiento F. L. and Brzezinski M. A. (2002) Silicic acid leakage from the Southern Ocean: A possible explanation for glacial atmospheric $\mathrm{pCO}_{2}$. Global Biogeochem. Cycles 16, 5-1-5-23. 
Matteuzzo M. C., Alexandre A., Varajão A. F. D. C., Volkmer-Ribeiro C., Almeida A. C. S., Varajão C. A. C., Vallet-Coulomb C., Sonzogni C. and Miche H. (2013) Assessing the relationship between the $\delta^{18} O$ signatures of siliceous sponge spicules and water in a tropical lacustrine environment (Minas Gerais, Brazil). Biogeosciences Discuss. 10, 12887-12918.

Mejia L. M., Isensee K., Méndez-Vicente A., Pisonero 7., Shimizu N., González C., Monteleone B. and Stoll H. (2013) B content and $\mathrm{Si} / \mathrm{C}$ ratios from cultured diatoms (Thalassiosira pseudonana and Thalassiosira weissflogii): Relationship to seawater pH and diatom carbon acquisition. Geochim. Cosmochim. Acta 123, 322-337. Available at: http://linkinghub.elsevier.com/retrieve/pii/ S001670371300344X.

Michalopoulos P., Aller R. C., and Reeder R. F. (2000) Conversion of diatoms to clays during early diagenesis in tropical, continental shelf muds. Geology 28, 1095-1098.

Michalopoulos P. and Aller R. C. (2004) Early diagenesis of biogenic silica in the Amazon delta: Alteration, authigenic clay formation, and storage. Geochim. Cosmochim. Acta 68, 1061-1085.

Needoba, 7.A., Waser, N.A., Harrison, P.J. and Calvert, S.E. (2003). Nitrogen isotope fractionation in 12 species of marine phytoplankton during growth on nitrate. Marine Ecology Progress Series, 255, 81-91.

Needoba, 7.A. and Harrison, P.J. (2004). Influence of low light and a light:dark cycle on NO-3 uptake, intracellular NO-3, and nitrogen isotope fractionation by marine phytoplankton. Fournal of Phycology, 40, 505-516.

Nelson D. M., Treguer P., Brzezinski M. A., Leynaert A. and Queguiner B. (1995) Production and dissolution of biogenic silica in the ocean: Revised global estimates, comparison with regional data and relationship to biogenic sedimentation limit. Global Biogeochem. Cycles 9, 359-372.

Pichevin L. E., Ganeshram R. S., Geibert W., Thunell R. and Hinton R. (2014) Silica burial enhanced by iron limitation in oceanic upwelling margins. Nat. Geosci. 7, 541-546.

Pichevin L., Ganeshram R. S., Reynolds B. C., Prahl F, Pedersen T. F., Thunell R. and McClymont E. L. (2012) Silicic acid biogeochemistry in the Gulf of California: Insights from sedimentary Si isotopes. Paleoceanography 27, 1-14.

Popp, B. N., Lawes E.A., Bidigare R.R., Dore J.E., Hanson K.L. and Wakeham, S.G. (1998). Effect of phytoplankton cell geometry on carbon isotopic fractionation. Geochimica et Cosmochimica Acta, 62, 69-77.

Ragueneau O., Tréguer P., Leynaert a., Anderson R. F., Brzezinski M. a., DeMaster D. F., Dugdale R. C., Dymond F., Fischer G., François R., Heinze C., Maier-Reimer E., Martin-fézéquel V., Nelson D. M. and Quéguiner B. (2000) A review of the Si cycle in the modern ocean: Recent progress and missing gaps in the application of biogenic opal as a paleoproductivity proxy. Glob. Planet. Change 26, 317-365.

Rahman S., Aller R. C. and Cochran 7. K. (2017) The Missing Silica Sink: Revisiting the Marine Sedimentary Si Cycle Using Cosmogenic 32Si. Global Biogeochem. Cycles 31, 1559-1578.

Rahman S., Tamborski F. F., Charette M. A. and Cochran F. K. (2019) Dissolved silica in the subterranean estuary and the impact of submarine groundwater discharge on the global marine silica budget. Mar. Chem. 208, 29-42. Available at: https://doi. org/10.1016/j.marchem.2018.11.006.

Ren H., Brunelle B. G., Sigman D. M. and Robinson R. S. (2013) Diagenetic aluminum uptake into diatom frustules and the preservation of diatom-bound organic nitrogen. Mar. Chem. 155, 92-101. Available at: http://dx.doi.org/10.1016/j. marchem.2013.05.016.

Robinson R. S., Brunelle B. G. and Sigman D. M. (2004) Revisiting nutrient utilization in the glacial Antarctic: Evidence from a new method for diatom-bound $\mathcal{N}$ isotopic analysis. Paleoceanography 19, 1-13.

Roepert, A. (2019): Imaging element distributions within small marine calcifiers: a NanoSIMS perspective. Utrecht Studies in Earth Sciences 201. ISBN 978-90-6266-560-0.

Rosenthal, Y., M. Dahan, and A. Shemesh (2000), Southern Ocean contributions to glacial-interglacial changes of atmospheric pCO2:An assessment of carbon isotope records in diatoms, Paleoceanography, 15, 65-75.

Sarthou G., Timmermans K. R., Blain S. and Tréguer P. (2005) Growth physiology and fate of diatoms in the ocean: a review. F. Sea Res. 53, 25-42. Available at: http://linkinghub.elsevier.com/retrieve/pii/S1385110104000644.

Schneider-Mor A., Yam R., Bianchi C., Kunz-Pirrung M., Gersonde R. and Shemesh A. (2005) Diatom stable isotopes, sea ice presence and sea surface temperature records of the past $640 \mathrm{ka}$ in the Atlantic sector of the Southern Ocean. Geophys. Res. Lett. 32, 1-4. 
Shemesh, A., Charles, C.D. and Fairbanks R.G. (1992). Oxygen isotopes in biogenic silica: global changes in ocean temperature and isotopic composition. Science, 256, 1434-1436.

Shemesh, A., Macko, S. A., Charles, C. D., and Rau, G. H. (1993). Isotopic evidence for reduced productivity in the glacial Southern Ocean. Science 262, 407-410. doi: 10.1126/science.262.5132.407

Shemesh A., Burckle L. H. and Hays F. D. (1995) Late Pleistocene oxygen isotope records of biogenic silica from the Atlantic sector of the Southern Ocean. Paleoceanography 10, 179-196.

Shemesh A., Mortlock R. A., Smith R. F. and Froelich P. N. (1988) Determination of Ge/Si in marine siliceous microfossils: Separation, cleaning and dissolution of diatoms and radiolaria. Mar. Chem. 25, 305-323.

Siever R. (1992) The silica cycle in the Precambrian. Geochim. Cosmochim. Acta 56, 3265-3272.

Sigman D. M., Altabet M. A., Francois R., McCorkle D. C. and Gaillard F. F. (1999) The isotopic composition of diatom-bound nitrogen in Southern Ocean sediments. Paleoceanography 14, 118-134.

Sims P. A., Mann D. G. and Medlin L. K. (2006) Evolution of the diatoms: Insights from fossil, biological and molecular data. Phycologia 45, 361-402.

Singer, A. F., and A. Shemesh (1995), Climatically linked carbon-isotope variation during the past 430,000 years in Southern-Ocean sediments, Paleoceanography,

Snelling A. M., Swann G. E. A., Pike F. and Leng M. F. (2014) Pliocene diatom and sponge spicule oxygen isotope ratios from the Bering Sea: Isotopic offsets and future directions. Clim. Past 10, 1837-1842.

Sorhannus U. (2007) A nuclear-encoded small-subunit ribosomal RNA timescale for diatom evolution. Mar. Micropaleontol. 65, 1-12.

Stoll H. M., Mendez-Vicente A., Abrevaya L., Anderson R. F., Rigual-Hernández A. S. and Gonzalez-Lemos S. (2016) Growth rate and size effect on carbon isotopic fractionation in diatom-bound organic matter in recent Southern Ocean sediments. Earth Planet. Sci. Lett. 1, 1-13. Available at: http://linkinghub.elsevier.com/retrieve/pii/S0012821X16305088.

Sutton F. N., André L., Cardinal D., Conley D. F., De Souza G. F., Dean F., Dodd F., Ehlert C., Ellwood M. F., Frings P. F., Grasse P., Hendry K., Leng M. F., Michalopoulos P., Panizzo V.N. and Swann G. E. A. (2018) A review of the stable isotope biogeochemistry of the global silicon cycle and its associated trace elements. Front. Earth Sci. $\mathbf{5}$.

Sutton F. N., Varela D. E., Brzezinski M. A. and Beucher C. P. (2013) Species-dependent silicon isotope fractionation by marine diatoms. Geochim. Cosmochim. Acta 104, 300-309. Available at: http://dx.doi.org/10.1016/j.gca.2012.10.057.

Swann G. E. A. and Leng M. F. (2009) A review of diatom 8180 in palaeoceanography. Quat. Sci. Rev. 28, 384-398.

Swann G. E. A., Maslin M. A., Leng M. F., Sloane H. F. and Haug G. H. (2006) Diatom 8180 evidence for the development of the modern halocline system in the subarctic northwest Pacific at the onset of major Northern Hemisphere glaciation. Paleoceanography 21, 1-12.

Takeda S. (1998) Influence of iron availability on nutrient consumption ratio of diatoms in oceanic waters. Nature 393, $774-777$.

Thamatrakoln K. and Hildebrand M. (2008) Silicon uptake in diatoms revisited: A model for saturable and nonsaturable uptake kinetics and the role of silicon transporters. Plant Physiol. 146, 1397-1407.

De Tommasi E., Gielis 7. and Rogato A. (2017) Diatom Frustule Morphogenesis and Function: a Multidisciplinary Survey. Mar. Genomics 35, 1-18. Available at: https://doi.org/10.1016/j.margen.2017.07.001.

Tréguer P. F. and De La Rocha C. L. (2013) The World Ocean Silica Cycle. Ann. Rev. Mar. Sci. 5, 477-501.

Tréguer P., Nelson D. M., Van Bennekom A. F., Demaster D. J., Leynaert A. and Quéguiner B. (1995) The silica balance in the world ocean: A reestimate. Science (80-.). 268, 375-379.

Tw B. S., Baines S. B., Fisher N. S., Vogt S., Facobsen C., Tovar-sanchez A. and San S. A. (2003) Qua nt ifying T ra ce Ele $m$ e nt s in I ndividua l Aqua tic Prot ist Ce lls w it h a Sync hrot ron X-ra y Fluore sc e nc e M ic roprobe. 75, 3806-3816.

Twining B. S. and Baines S. B. (2013) The trace metal composition of marine phytoplankton. Ann. Rev. Mar. Sci. 5, 191-215.

Tyler F. F., Sloane H. F., Rickaby R. E. M., Cox E. F. and Leng M. F. (2017) Post-mortem oxygen isotope exchange within cultured diatom silica. Rapid Commun. Mass Spectrom. Available at: http://doi.wiley.com/10.1002/rcm. 7954.

Uriz M. F. (2006) Mineral skeletogenesis in sponges. Can. F. Zool. 84, 322-356.

Uriz M. J., Turon X., Becerro M. A. and Agell G. (2003) Siliceous spicules and skeleton frameworks in sponges: Origin, diversity, ultrastructural patterns, and biological functions. Microsc. Res. Tech. 62, 279-299. 
Vrieling E., Poort L., Beelen T. and Gieskes W. (1999) Growth and silica content of the diatoms thalassiosira weissflogii and navicula salinarum at different salinities and enrichments with aluminium. Eur. F. Phycol. 34, 307-316.

Walker, F.C.G., Hays, P.B., Kasting, F.F., 1981. A negative feedback mechanism for the long-term stabilization of Earth's surface temperature. 7. Geophys. Res. 86, 9776-978

Wetzel F., de Souza G. F. and Reynolds B. C. (2014) What controls silicon isotope fractionation during dissolution of diatom opal? Geochim. Cosmochim. Acta 131, 128-137. Available at: http://dx.doi.org/10.1016/j.gca.2014.01.028.

Wille, M., Sutton, F., Ellwood, M. F., Sambridge, M., Maher, W., Eggins, S., et al. (2010). Silicon isotopic fractionation in marine sponges: a new model for understanding silicon isotopic variations in sponges. Earth Planet. Sci. Lett. 292, 281-289. doi: 10.1016/j.epsl.2010.01.036

Yuan P., Liu D., Zhou Funming, Tian Q. Song Y., Wei H., Wang S., Zhou Fieyu, Deng L. and Du P. (2019) Identification of the occurrence of minor elements in the structure of diatomaceous opal using FIB and TEM-EDS. Am. Mineral. 104, 1323-1335. Zhang Y., Obata H. and Nozaki Y. (2004) Silver in the Pacific Ocean and the Bering Sea. Geochem. F. 38, 623-633.

Zheng X. Y., Beard B. L. and Fohnson C. M. (2019) Constraining silicon isotope exchange kinetics and fractionation between aqueous and amorphous Si at room temperature. Geochim. Cosmochim. Acta 253, 267-289. Available at: https://doi.org/10.1016/j. gca.2019.03.031. 


\section{Chapter 2}

Arellano-Torres E., Pichevin L. E. and Ganeshram R. S. (2011) High-resolution opal records from the eastern tropical Pacific provide evidence for silicic acid leakage from HNLC regions during glacial periods. Quat. Sci. Rev. 30, 1112-1121. Available at: http:// dx.doi.org/10.1016/j.quascirev.2011.02.002.

Van Bennekom A. F., Buma A. G. F. and Nolting R. F. (1991) Dissolved aluminium in the Weddell-Scotia Confluence and effect of Al on the dissolution kinetics of biogenic silica. Mar. Chem. 35, 423-434.

van Bennekom A. F., Fred Jansen F. H., van der Gaast S. F., van Iperen 7. M. and Pieters 7. (1989) Aluminium-rich opal: an intermediate in the preservation of biogenic silica in the Zaire (Congo) deep-sea fan. Deep Sea Res. Part A, Oceanogr. Res. Pap. 36, $173-190$.

Bridoux M. C. and Ingalls A. E. (2010) Structural identification of long-chain polyamines associated with diatom biosilica in a Southern Ocean sediment core. Geochim. Cosmochim. Acta 74, 4044-4057. Available at: http://dx.doi.org/10.1016/j.gca.2010.04.010.

Cappellen P. Van and Qiu L. (1997) Biogenic silica dissolution in sediments of the Southern Ocean: II. Kinetics. Deep. Res. Part II Top. Stud. Oceanogr. 44, 1129-1149.

Crosta X., Sturm A., Armand L. and Pichon F. F. (2004) Late Quaternary sea ice history in the Indian sector of the Southern Ocean as recorded by diatom assemblages. Mar. Micropaleontol. 50, 209-223.

DeMaster D. F. (2002) The accumulation and cycling of biogenic silica in the Southern Ocean: Revisiting the marine silica budget. Deep. Res. Part II Top. Stud. Oceanogr. 49, 3155-3167.

Dixit S., Van Cappellen P. and Van Bennekom A. F. (2001) Processes controlling solubility of biogenic silica and pore water build-up of silicic acid in marine sediments. Mar. Chem. 73, 333-352.

Dymond, F., Lyle, M., 1985. Flux comparisons between sediment and sediment traps in the eastern tropical Pacific: implications for atmospheric CO variations during the Plesitocene. Limnol. Oceanogr. 30, 699-712.

Ellwood M. F. and Hunter K. A. (1999) Determination of the Zn/Si ratio in diatom opal: A method for the separation, cleaning and dissolution of diatoms. Mar. Chem. 66, 149-160.

Hendry K. R. and Rickaby R. E. M. (2008) Opal (Zn/Si) ratios as a nearshore geochemical proxy in coastal Antarctica. Paleoceanography 23, 1-12.

Hutchins D. A. and Bruland K. W. (1998) Iron-limited growth and Si:N ratios in a costal upwelling regime. Nature 393, 561-564.

Ingall E. D., Diaz F. M., Longo A. F., Oakes M., Finney L., Vogt S., Lai B., Tager P. L., Twining B. S. and Brandes F. A. (2013) Role of biogenic silica in the removal of iron from the Antarctic seas. Nat. Commun. 4, 1981. Available at: http://wrere.nature. com/doifinder/10.1038/ncomms2981.

Koning E., Gehlen M., Flank A. M., Calas G. and Epping E. (2007) Rapid post-mortem incorporation of aluminum in diatom frustules. Evidence from chemical and structural analyses. Mar. Chem. 103, 97-111.

De La Rocha C. L., Brzezinski M. A. and DeNiro M. F. (1997) Fractionation of silicon isotopes by marine diatoms during biogenic silica formation. Geochim. Cosmochim. Acta 61, 5051-5056.

Morley D. W., Leng M. F., Mackay A. W., Sloane H. F., Rioual P. and Battarbee R. W. (2004) Cleaning of lake sediment samples for diatom oxygen isotope analysis. F. Paleolimnol. 31, 391-401.

Pichevin L. E., Ganeshram R. S., Geibert W., Thunell R. and Hinton R. (2014) Silica burial enhanced by iron limitation in oceanic upwelling margins. Nat. Geosci. 7, 541-546.

Pichevin L., Ganeshram R. S., Reynolds B. C., Prahl F., Pedersen T. F., Thunell R. and McClymont E. L. (2012) Silicic acid biogeochemistry in the Gulf of California: Insights from sedimentary Si isotopes. Paleoceanography 27, 1-14.

Ren H., Brunelle B. G., Sigman D. M. and Robinson R. S. (2013) Diagenetic aluminum uptake into diatom frustules and the preservation of diatom-bound organic nitrogen. Mar. Chem. 155, 92-101. Available at: http://dx.doi.org/10.1016/j. marchem.2013.05.016.

Robinson R. S., Brunelle B. G. and Sigman D. M. (2004) Revisiting nutrient utilization in the glacial Antarctic: Evidence from a new method for diatom-bound $\mathcal{N}$ isotopic analysis. Paleoceanography 19, 1-13. 
Shemesh A., Mortlock R. A., Smith R. F. and Froelich P. N. (1988) Determination of Ge/Si in marine siliceous microfossils: Separation, cleaning and dissolution of diatoms and radiolaria. Mar. Chem. 25, 305-323.

Sigman, D.M., Boyle, E.A., 2000. Glacial/interglacial variations in atmospheric carbon dioxide. Nature 407, 859-869.Sorhannus U. (2007) A nuclear-encoded small-subunit ribosomal RNA timescale for diatom evolution. Mar. Micropaleontol. 65, 1-12.

Takeda S. (1998) Influence of iron availability on nutrient consumption ratio of diatoms in oceanic waters. Nature 393, $774-777$.

Yuan P., Liu D., Zhou Junming, Tian Q. Song Y., Wei H., Wang S., Zhou Fieyu, Deng L. and Du P. (2019) Identification of the occurrence of minor elements in the structure of diatomaceous opal using FIB and TEM-EDS. Am. Mineral. 104, 1323-1335. 


\section{Chapter 3}

Bridoux M. C. and Ingalls A. E. (2010) Structural identification of long-chain polyamines associated with diatom biosilica in a Southern Ocean sediment core. Geochim. Cosmochim. Acta 74, 4044-4057. Available at: http://dx.doi.org/10.1016/j.gca.2010.04.010.

Des Combes H. 7., Esper O., De La Rocha C. L., Abelmann A., Gersonde R., Yam R. and Shemesh A. (2008) Diatom 813C, 815N, and $C / \mathcal{N}$ since the last glacial maximum in the southern ocean: potential impact of species composition. Paleoceanography 23, 1-12.

Hecky RE, Mopper K, Kilham P, Degens ET (1973) The amino acid and sugar composition of diatom cell walls. Mar Biol 19:323

Hendry K. R. and Rickaby R. E. M. (2008) Opal (Zn/Si) ratios as a nearshore geochemical proxy in coastal Antarctica.

Paleoceanography 23, 1-12.

Hildebrand M., Lerch S. F. L. and Shrestha R. P. (2018) Understanding diatom cell wall silicification-moving forward. Front. Mar. Sci. 5, 1-19.

Horn M. G., Beucher C. P., Robinson R. S. and Brzezinski M. A. (2011a) Southern ocean nitrogen and silicon dynamics during the last deglaciation. Earth Planet. Sci. Lett. 310, 334-339. Available at: http://dx.doi.org/10.1016/j.epsl.2011.08.016.

Horn M. G., Robinson R. S., Rynearson T. A. and Sigman D. M. (2011b) Nitrogen isotopic relationship between diatom-bound and bulk organic matter of cultured polar diatoms. Paleoceanography 26, 1-12.

Ingalls A. E., Lee C., Wakeham S. G. and Hedges 7. I. (2003) The role of biominerals in the sinking flux and preservation of amino acids in the Southern Ocean along $170^{\circ}$ W. Deep. Res. Part II Top. Stud. Oceanogr. 50, 713-738.

Kröger, N., Lorenz, S., Brunner, E. and Sumper, M. (2002). Self-assembly of highly phosphorylated silaffins and their function in biosilica morphogenesis. Science, 298, 584-586.

Kröger $\mathcal{N}$. and Poulsen $\mathcal{N}$. (2008) Diatoms - From Cell Wall Biogenesis to Nanotechnology. Annu. Rev. Genet. 42, 83-107. Available at: http://wrere.annualreviews.org/doi/10.1146/annurev.genet.41.110306.130109.

De La Rocha C. L. (2006) Opal-based isotopic proxies of paleoenvironmental conditions. Global Biogeochem. Cycles 20, 1-11.

Morley D. W., Leng M. J., Mackay A. W., Sloane H. F., Rioual P. and Battarbee R. W. (2004) Cleaning of lake sediment samples for diatom oxygen isotope analysis. F. Paleolimnol. 31, 391-401.

Pichevin L. E., Ganeshram R. S., Geibert W., Thunell R. and Hinton R. (2014) Silica burial enhanced by iron limitation in oceanic upwelling margins. Nat. Geosci. 7, 8-13. Available at: http://wwerenature.com/doifinder/10.1038/ngeo2181.

Pichevin L., Ganeshram R. S., Reynolds B. C., Prahl F., Pedersen T. F., Thunell R. and McClymont E. L. (2012) Silicic acid biogeochemistry in the Gulf of California: Insights from sedimentary Si isotopes. Paleoceanography 27, 1-14.

Polerecky L., Adam B., Milucka F., Musat N., Vagner T. and Kuypers M. M. M. (2012)Look@NanoSIMS-a tool for the analysis of nanoSIMS data in environmental microbiology. Environ. Microbiol. 14, 1009-1023.

Ren H., Brunelle B. G., Sigman D. M. and Robinson R. S. (2013) Diagenetic aluminum uptake into diatom frustules and the preservation of diatom-bound organic nitrogen. Mar. Chem. 155, 92-101. Available at: http://dx.doi.org/10.1016/j. marchem.2013.05.016.

Robinson R. S., Brunelle B. G. and Sigman D. M. (2004) Revisiting nutrient utilization in the glacial Antarctic: Evidence from a new method for diatom-bound $\mathcal{N}$ isotopic analysis. Paleoceanography 19, 1-13.

Robinson R. S., Brzezinski M. A., Beucher C. P., Horn M. G. S. and Bedsole P. (2014) The changing roles of iron and vertical mixing in regulating nitrogen and silicon cycling in the Southern Ocean over the last glacial cycle. Paleoceanography 29, 1179-1195.

Shemesh, A., Macko, S. A., Charles, C. D., and Rau, G. H. (1993). Isotopic evidence for reduced productivity in the glacial Southern Ocean. Science 262, 407-410. doi: 10.1126/science.262.5132.407

Sigman D. M., Altabet M. A., Francois R., McCorkle D. C. and Gaillard F. F. (1999) The isotopic composition of diatom-bound nitrogen in Southern Ocean sediments. Paleoceanography 14, 118-134.

Tesson B. and Hildebrand M. (2013) Characterization and Localization of Insoluble Organic Matrices Associated with Diatom Cell Walls: Insight into Their Roles during Cell Wall Formation. PLoS One $\mathbf{8}$.

Thamatrakoln K. and Hildebrand M. (2008) Silicon uptake in diatoms revisited: A model for saturable and nonsaturable uptake kinetics and the role of silicon transporters. Plant Physiol. 146, 1397-1407. 


\section{Chapter 4}

Bidle K. and Azam F. (2001) Bacterial control of silicon regeneration from diatom detritus: Significance of bacterial ectohydrolases and species identity. Limnol. Oceanogr. 46, 1606-1623.

Bidle K. D. and Azam F. (1999) Accelerated dissolution of diatom silica by marine bacterial assemblages. Nature 397, 508-512.

Bidle K. D., Brzezinski M. A., Long R. A., Fones F. L. and Azam F. (2003) Diminished efficiency in the oceanic silica pump caused by bacteria-mediated silica dissolution. Limnol. Oceanogr. 48, 1855-1868.

Brandriss M. E., O'Neil F. R., Edlund M. B. and Stoermer E. F. (1998) Oxygen isotope fractionation between diatomaceous silica and water. Geochim. Cosmochim. Acta 62, 1119-1125.

Chapligin B., Leng M. J., Webb E., Alexandre A., Dodd F. P., Ijiri A., Lücke A., Shemesh A., Abelmann A., Herzschuh U., Longstaffe F. F., Meyer H., Moschen R., Okazaki 1., Rees N. H., Sharp Z. D., Sloane H. F., Sonzogni C., Swann G. E. A., Sylvestre F., Tyler 7. F. and Yam R. (2011) Inter-laboratory comparison of oxygen isotope compositions from biogenic silica. Geochim. Cosmochim. Acta 75, 7242-7256.

Dodd F. P. and Sharp Z. D. (2010) A laser fluorination method for oxygen isotope analysis of biogenic silica and a new oxygen isotope calibration of modern diatoms in freshwater environments. Geochim. Cosmochim. Acta 74, 1381-1390. Available at: http://dx.doi. org/10.1016/j.gca.2009.11.023.

Dodd F. P., Sharp Z. D., Fawcett P. F., Brearley A. F. and McCubbin F. M. (2012) Rapid post-mortem maturation of diatom silica oxygen isotope values. Geochemistry, Geophys. Geosystems 13, 1-12.

Dodd F. P., Wiedenheft W. and Schwartz F. M. (2017) Dehydroxylation and diagenetic variations in diatom oxygen isotope values. Geochim. Cosmochim. Acta 199, 185-195. Available at: http://dx.doi.org/10.1016/j.gca.2016.11.034.

Fripiat 7. F., Letellier M. and Levitz P. (1984) Interaction of water with clay surfaces. Philos. Trans. R. Soc. London 299, $287-299$. Gendron-Badou A., Coradin T., Maquet 7., Fröhlich F. and Livage 7. (2003) Spectroscopic characterization of biogenic silica. F. Non. Cryst. Solids 316, 331-337.

Hendry K. R., Georg R. B., Rickaby R. E. M., Robinson L. F. and Halliday A. N. (2010) Deep ocean nutrients during the Last Glacial Maximum deduced from sponge silicon isotopic compositions. Earth Planet. Sci. Lett. 292, 290-300. Available at: http://dx.doi. org/10.1016/j.epsl.2010.02.005.

Hendry K. R. and Robinson L. F. (2012) The relationship between silicon isotope fractionation in sponges and silicic acid concentration: Modern and core-top studies of biogenic opal. Geochim. Cosmochim. Acta 81, 1-12. Available at: http://dx.doi.org/10.1016/j. gca.2011.12.010.

Hildebrand M., York E., Kelz F. I., Davis A. K., Frigeri L. G., Allison D. P. and Doktycz M. F. (2006) Nanoscale control of silica morphology and three-dimensional structure during diatom cell wall formation. F. Mater. Res. 21, 2689-2698.

Kammer M., Hedrich R., Ehrlich H., Popp F., Brunner E. and Krafft C. (2010) Spatially resolved determination of the structure and composition of diatom cell walls by Raman and FTIR imaging. Anal. Bioanal. Chem. 398, 509-517.

Knauth L. P. and Epstein S. (1982) The nature of water in hydrous silica. Am. Mineral. 67, 510-520.

Knauth L. P. (1973) Oxygen and hydrogen isotope ratios in cherts and related rocks. Ph.D. thesis, California Institute of Technology.

Kopp C., Meibom A., Beyssac O., Stolarski F., Djediat S., Szlachetko F. and Domart-Coulon I. (2011) Calcareous sponge biomineralization: Ultrastructural and compositional heterogeneity of spicules in Leuconia johnstoni Carter, 1871. 7. Struct. Biol. 173, 99-109. Available at: http://dx.doi.org/10.1016/j.jsb.2010.07.006.

Labeyrie L. (1974) New approach to surface seawater paleotemperatures using '80/'60 ratios in silica diatom frustules. Nature 248,4042.

Leclerc A. F. and Labeyrie L. (1987) Temperature dependence of the oxygen isotopic fractionation between diatom silica and water. Earth Planet. Sci. Lett. 84, 69-74.

Leng M. F. and Barker P. A. (2006) A review of the oxygen isotope composition of lacustrine diatom silica for palaeoclimate reconstruction. Earth-Science Rev. 75, 5-27.

Lewin 7. C. (1961) The dissolution of silica from diatom walls. Geochim. Cosmochim. Acta 21, 182-198.

Loucaides S., Behrends T. and Cappellen P. Van (2010) Reactivity of biogenic silica: Surface versus bulk charge density. Geochim. Cosmochim. Acta 74, 517-530. Available at: http://dx.doi.org/10.1016/j.gca.2009.10.038. 
Matheney R. K. and Knauth L. P. (1989) Oxygen-isotope fractionation between marine biogenic silica and seawater. Geochim. Cosmochim. Acta 53, 3207-3214.

Matteuzzo M. C., Alexandre A., Varajão A. F. D. C., Volkmer-Ribeiro C., Almeida A. C. S., Varajão C. A. C., Vallet-Coulomb C., Sonzogni C. and Miche H. (2013) Assessing the relationship between the $\delta^{18} O$ signatures of siliceous sponge spicules and water in a tropical lacustrine environment (Minas Gerais, Brazil). Biogeosciences Discuss. 10, 12887-12918.

McMillan P., Piriou B. and Navrotsky A. (1982) A Raman spectroscopic study of glasses along the joins silica-calcium aluminate, silicasodium aluminate, and silica-potassium aluminate. Geochim. Cosmochim. Acta 46, 2021-2037.

Menicucci A. F., Spero H. F., Matthews F. and Parikh S. F. (2017) Influence of exchangeable oxygen on biogenic silica oxygen isotope data. Chem. Geol. 466, 710-721. Available at: http://dx.doi.org/10.1016/j.chemgeo.2017.07.020.

Mills R. (1973) Self-diffusion in normal and heavy water in the range 1-45?? F. Phys. Chem. 77, 685-688.

Moschen R., Lücke A., Parplies 7., Radtke U. and Schleser G. H. (2006) Transfer and early diagenesis of biogenic silica oxygen isotope signals during settling and sedimentation of diatoms in a temperate freshwater lake (Lake Holzmaar, Germany). Geochim. Cosmochim. Acta 70, 4367-4379.

Moschen R., Lücke A. and Schleser G. H. (2005) Sensitivity of biogenic silica oxygen isotopes to changes in surface water temperature and palaeoclimatology. Geophys. Res. Lett. 32, 1-4.

Müller W. E. G., Li J., Schröder H. C., Qiao L. and Wang X. (2007) The unique skeleton of siliceous sponges (Porifera; Hexactinellida and Demospongiae) that evolved first from the Urmetazoa during the Proterozoic: A review. Biogeosciences 4, $219-232$.

Polerecky L., Adam B., Milucka F., Musat N., Vagner T. and Kuypers M. M. M. (2012)Look@NanoSIMS-a tool for the analysis of nanoSIMS data in environmental microbiology. Environ. Microbiol. 14, 1009-1023.

Rohling, E., Cooke, S., 1999. Stable oxygen and carbon isotopes in foraminiferal carbonate shells. In: Sen Gupta, B. (Ed.), Modern Foraminifera. Kluwer, Dordrecht, pp. 239-258.

Sandford F. (2003) Physical and chemical analysis of the siliceous skeletons in six sponges of two groups (demospongiae and hexactinellida). Microsc. Res. Tech. 62, 336-355.

Sato K., Fujimoto K., Nakata M. and Hatta T. (2011) Diffusion-reaction of water molecules in angstrom pores as basic mechanism of biogenic quartz formation. F. Phys. Chem. C115, 18131-18135.

Snelling A. M., Swann G. E. A., Pike F. and Leng M. F. (2014) Pliocene diatom and sponge spicule oxygen isotope ratios from the Bering Sea: Isotopic offsets and future directions. Clim. Past 10, 1837-1842.

Schmidt M., Botz R., Rickert D., Bohrmann G., Hall S. R. and Mann S. (2001) Oxygen isotopes of marine diatoms and relations to opal-A maturation. Geochim. Cosmochim. Acta 65, 201-211.

Shemesh, A., Macko, S. A., Charles, C. D., and Rau, G. H. (1993). Isotopic evidence for reduced productivity in the glacial Southern Ocean. Science 262, 407-410. doi: 10.1126/science.262.5132.407

Smith A. C., Leng M. F., Swann G. E. A., Barker P. A., Mackay A. W., Ryves D. B., Sloane H. F., Chenery S. R. N. and Hems M. (2016) An experiment to assess the effects of diatom dissolution on oxygen isotope ratios. Rapid Commun. Mass Spectrom. 30, 293300 .

Swann G. E. A. and Leng M. F. (2009) A review of diatom 8180 in palaeoceanography. Quat. Sci. Rev. 28, 384-398.

Swann G. E. A., Maslin M. A., Leng M. F., Sloane H. F. and Haug G. H. (2006) Diatom 8180 evidence for the development of the modern halocline system in the subarctic northwest Pacific at the onset of major Northern Hemisphere glaciation. Paleoceanography 21, 1-12.

Terpstra P., Combes D. and Zwick A. (1990) Effect of salts on dynamics of water: A Raman spectroscopy study. 7. Chem. Phys. 92, 65-70.

De Tommasi E., Congestri R., Dardano P., De Luca A. C., Managò S., Rea I. and De Stefano M. (2018) UV-shielding and wavelength conversion by centric diatom nanopatterned frustules. Sci. Rep. 8, 1-14.

Tyler F. F., Sloane H. F., Rickaby R. E. M., Cox E. F. and Leng M. F. (2017) Post-mortem oxygen isotope exchange within cultured diatom silica. Rapid Commun. Mass Spectrom. 31, 1749-1760.

Wojdyr M. Fityk: a general-purpose peak fitting program. 7. Appl. Crystallogr. 2010, 43, 1126-1128. 
Zachos, J.C., Pagani, M., Sloan, L., Thomas, E., Billups, K., 2001. Trends, rhythms, and aberrations in global climate 65 Ma to present. Science 292, 686-693.

Zhuravlev L. T. (2000) The surface chemistry of amorphous silica. Zhuravlev model. Colloids Surfaces A Physicochem. Eng. Asp. 173, $1-38$. 


\section{Chapter 5}

Van Bennekom A. F., Buma A. G. F. and Nolting R. F. (1991) Dissolved aluminium in the Weddell-Scotia Confluence and effect of Al on the dissolution kinetics of biogenic silica. Mar. Chem. 35, 423-434.

Brandriss M. E., O'Neil F. R., Edlund M. B. and Stoermer E. F. (1998) Oxygen isotope fractionation between diatomaceous silica and water. Geochim. Cosmochim. Acta 62, 1119-1125.

Brewer T. S., Leng M. F., Mackay A. W., Lamb A. L., Tyler F. F. and Marsh N. G. (2008) Unravelling contamination signals in biogenic silica oxygen isotope composition: the role of major and trace element geochemistry. F. Quat. Sci. 23, 321-330.

Cappellen P. Van and Qiu L. (1997) Biogenic silica dissolution in sediments of the Southern Ocean: II. Kinetics. Deep. Res. Part II Top. Stud. Oceanogr. 44, 1129-1149.

DeMaster D. F. (2002) The accumulation and cycling of biogenic silica in the Southern Ocean: Revisiting the marine silica budget. Deep. Res. Part II Top. Stud. Oceanogr. 49, 3155-3167.

Dixit S., Van Cappellen P. and Van Bennekom A. F. (2001) Processes controlling solubility of biogenic silica and pore water build-up of silicic acid in marine sediments. Mar. Chem. 73, 333-352.

Dodd 7. P. and Sharp Z. D. (2010) A laser fluorination method for oxygen isotope analysis of biogenic silica and a new oxygen isotope calibration of modern diatoms in freshwater environments. Geochim Cosmochim. Acta 74, 1381-1390. Available at: http://dx.doi. org/10.1016/j.gca.2009.11.023.

Dodd F. P., Sharp Z. D., Fawcett P. F., Brearley A. F. and McCubbin F. M. (2012) Rapid post-mortem maturation of diatom silica oxygen isotope values. Geochemistry, Geophys. Geosystems 13, 1-12.

Dodd 7. P., Wiedenheft W. and Schwartz F. M. (2017) Dehydroxylation and diagenetic variations in diatom oxygen isotope values. Geochim. Cosmochim. Acta 199, 185-195. Available at: http://dx.doi.org/10.1016/j.gca.2016.11.034.

Gendron-Badou A., Coradin T., Maquet F., Fröhlich F. and Livage 7. (2003) Spectroscopic characterization of biogenic silica. F. Non. Cryst. Solids 316, 331-337.

Iler R. K. (1973) Effect of adsorbed alumina on the solubility of amorphous silica in water. F. Colloid Interface Sci. 43, 399-408.

Iler, R.K., 1979. The chemistry of silica. Wiley-Interscience, New Tork.

Knauth L. P. (1973) Oxygen and hydrogen isotope ratios in cherts and related rocks. Ph.D. thesis, California Institute of Technology.

Koning E., Gehlen M., Flank A. M., Calas G. and Epping E. (2007) Rapid post-mortem incorporation of aluminum in diatom frustules: Evidence from chemical and structural analyses. Mar. Chem. 103, 97-111.

Labeyrie, L.D., 1979. La composition isotopique de l'oxygene de la silice des valves de diatomees. Mise au point d'une nouvelle methode de palaeo-climatologie. Diss. Universitie de Paris XI (in French).

Labeyrie L. D. and Fuillet A. (1982) Oxygen isotopic exchangeability of diatom valve silica; interpretation and consequences for paleoclimatic studies. Geochim. Cosmochim. Acta 46, 967-975.

Leclerc A. F. and Labeyrie L. (1987) Temperature dependence of the oxygen isotopic fractionation between diatom silica and water. Earth Planet. Sci. Lett. 84, 69-74.

Leng M., Barnker P., Greenwood P., Roberts N. and Reed F. (2001) Oxygen isotope analysis of diatom silica and authigenic calcite from Lake Pinarbasi, Turkey. F. Paleolimnol. 25, 343-349.

Leng M. F. and Barker P. A. (2006) A review ofthe oxygen isotope composition oflacustrine diatom silica for palaeoclimate reconstruction. Earth-Science Rev. 75, 5-27.

Leng, M.F., Sloane, H.F., 2008. Combined oxygen and silicon isotope analysis of biogenic silica. Fournal of Quaternary Science 23, 313-319.

Lewin 7. C. (1961) The dissolution of silica from diatom walls. Geochim. Cosmochim. Acta 21, 182-198.

Loucaides S., Behrends T. and Cappellen P. Van (2010) Reactivity of biogenic silica: Surface versus bulk charge density. Geochim. Cosmochim. Acta 74, 517-530. Available at: http://dx.doi.org/10.1016/j.gca.2009.10.038.

Matheney R. K. and Knauth L. P. (1989) Oxygen-isotope fractionation between marine biogenic silica and seawater. Geochim. Cosmochim. Acta 53, 3207-3214. 
Michalopoulos P. and Aller R. C. (2004) Early diagenesis of biogenic silica in the Amazon delta: Alteration, authigenic clay formation, and storage. Geochim. Cosmochim. Acta 68, 1061-1085.

Moschen R., Lücke A., Parplies F., Radtke U. and Schleser G. H. (2006) Transfer and early diagenesis of biogenic silica oxygen isotope signals during settling and sedimentation of diatoms in a temperate freshwater lake (Lake Holzmaar, Germany). Geochim. Cosmochim. Acta 70, 4367-4379.

Polerecky L., Adam B., Milucka F., Musat.N., Vagner T. and Kuypers M. M. M. (2012) Look@NanoSIMS-a tool for the analysis of nanoSIMS data in environmental microbiology. Environ. Microbiol. 14, 1009-1023.

Ren H., Brunelle B. G., Sigman D. M. and Robinson R. S. (2013) Diagenetic aluminum uptake into diatom frustules and the preservation of diatom-bound organic nitrogen. Mar. Chem. 155, 92-101. Available at: http://dx.doi.org/10.1016/j. marchem.2013.05.016.

Rickert D., Schlüter M. and Wallmann K. (2002) Dissolution kinetics of biogenic silica from the water column to the sediments. Geochim. Cosmochim. Acta 66, 439-455.

Rohling, E., Cooke, S., 1999. Stable oxygen and carbon isotopes in foraminiferal carbonate shells. In: Sen Gupta, B. (Ed.), Modern Foraminifera. Kluwer, Dordrecht, pp. 239-258.

Schmidt M., Botz R., Rickert D., Bohrmann G., Hall S. R. and Mann S. (2001) Oxygen isotopes of marine diatoms and relations to opal-A maturation. Geochim. Cosmochim. Acta 65, 201-211.

Shemesh, A., Charles, C.D. and Fairbanks R.G. (1992). Oxygen isotopes in biogenic silica: global changes in ocean temperature and isotopic composition. Science, 256, 1434-1436.

Smith A. C., Leng M. F., Swann G. E. A., Barker P. A., Mackay A. W., Ryves D. B., Sloane H. F., Chenery S. R. N. and Hems M. (2016) An experiment to assess the effects of diatom dissolution on oxygen isotope ratios. Rapid Commun. Mass Spectrom. 30, 293300 .

Swann, G.E.A., Leng, M.F., Sloane, H.J., Maslin, M.A., 2008. Isotope offsets in marine diatom d18O over the last 200 ka. Fournal of Quaternary Science 23, 389-400.

Swann G. E. A. and Leng M. F. (2009) A review of diatom 8180 in palaeoceanography. Quat. Sci. Rev. 28, 384-398.

Zachos, F.C., Pagani, M., Sloan, L., Thomas, E., Billups, K., 2001. Trends, rhythms, and aberrations in global climate 65 Ma to present. Science 292, 686-693.

Zhuravlev L. T. (2000) The surface chemistry of amorphous silica. Zhuravlev model. Colloids Surfaces A Physicochem. Eng. Asp. 173, $1-38$. 


\section{Chapter 6}

Dyar M. D., Wiedenbeck M., Robertson D., Cross L. R., Delaney 7. S., Ferguson K., Francis C. A., Grew E. S., Guidotti C. V., Hervig R. L., Hughes F. M., Husler F., Leeman W., McGuire A. V., Rhede D., Rothe H., Paul R. L., Richards I. and Yates M. (2001) Reference minerals for the microanalysis of light elements. Geostand. Newsl. 25, 441-463.

Fayek M. (2009) Secondary Ion Mass Spectrometry in the Earth Sciences: Gleaning the Big Picture From a Small Spot. Mineral. Assoc. Canada Short Course Ser. 41, 22-23.

Gabitov R. I., Gagnon A. C., Guan 1., Eiler F. M. and Adkins F. F. (2013) Accurate Mg/Ca, Sr/Ca, and Ba/Ca ratio measurements in carbonates by SIMS and NanoSIMS and an assessment of heterogeneity in common calcium carbonate standards. Chem. Geol. 356, 94-108. Available at: http://dx.doi.org/10.1016/j.chemgeo.2013.07.019.

Geerken E., de Nooïer L. F., Roepert A., Polerecky L., King H. E. and Reichart G. F. (2019) Element banding and organic linings within chamber walls of two benthic foraminifera. Sci. Rep. 9, 1-15. Available at: http://dx.doi.org/10.1038/s41598-019-40298-y.

Hoppe P., Cohen S. and Meibom A. (2013) NanoSIMS: Technical Aspects and Applications in Cosmochemistry and Biological Geochemistry. Geostand. Geoanalytical Res. 37, 111-154. Available at: http://doi.wiley.com/10.1111/j.1751908X.2013.00239.x.

Morley D. W., Leng M. F., Mackay A. W., Sloane H. F., Rioual P. and Battarbee R. W. (2004) Cleaning of lake sediment samples for diatom oxygen isotope analysis. F. Paleolimnol. 31, 391-401.

Nuñez F., Renslow R., CliffF. B. and Anderton C. R. (2018) NanoSIMS for biological applications: Current practices and analyses. Biointerphases 13, 03 B301.

Osterholz H., Simon H., Beck M., Maerz F., Rackebrandt S., Brumsack H.-F., Feudel U. and Simon M. (2014) Impact of diatom growth on trace metal dynamics (Mn, Mo, V, U). F. Sea Res. 87, 35-45. Available at: http://wrwresciencedirect.com/science/ article/pii/S1385110113002554.

Polerecky L., Adam B., Milucka F., Musat N., Vagner T. and Kuypers M. M. M. (2012)Look@NanoSIMS-a tool for the analysis of nanoSIMS data in environmental microbiology. Environ. Microbiol. 14, 1009-1023.

Roepert, A. (2019): Imaging element distributions within small marine calcifiers: a NanoSIMS perspective. Utrecht Studies in Earth Sciences 201. ISBN 978-90-6266-560-0

Robinson R. S., Brunelle B. G. and Sigman D. M. (2004) Revisiting nutrient utilization in the glacial Antarctic: Evidence from a new method for diatom-bound $\mathcal{N}$ isotopic analysis. Paleoceanography 19, 1-13.

Shimizu, N. (2019). Big picture geochemistry from microanalyses my four-decade odyssey inSIMS. Geochemical Perspectives, 8(1).

Snelling A. M., Swann G. E. A., Leng M. F. and Pike F. (2013) A Micro-manipulation Technique for the Purification of Diatoms for Isotope and Geochemical Analysis. Silicon 5, 13-17.

Stoll H. M. and Shimizu N. (2009) Micropicking of nannofossils in preparation for analysis by secondary ion mass spectrometry. Nat. Protoc. 4, 1038-43. Available at: http://wrere.ncbi.nlm.nih.gov/pubmed/19536271. 


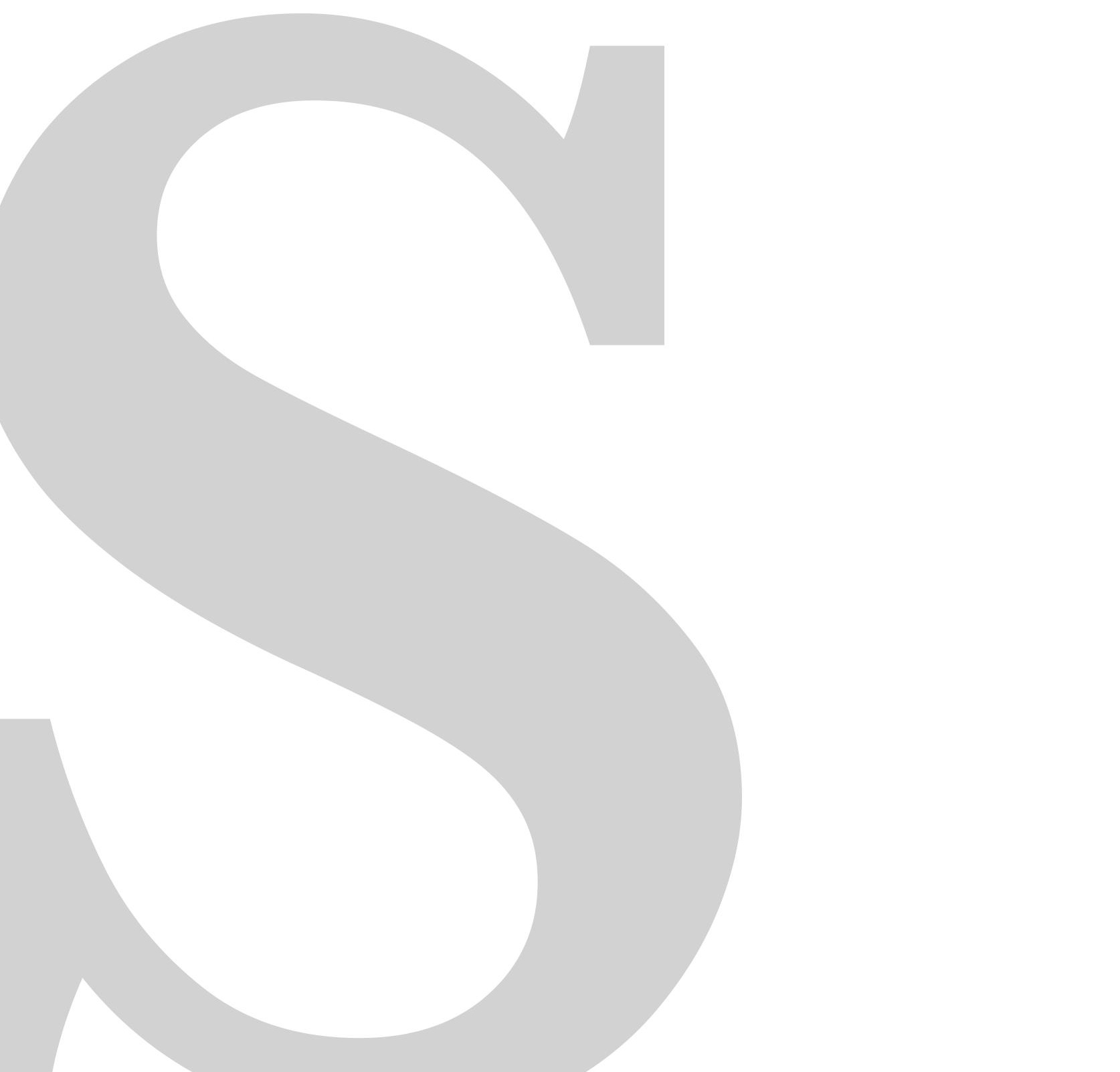




\section{Nederlandse Samenvatting (Summary in Dutch)}

\section{Achtergrond}

Silicium is het zevende meest voorkomende element in het universum en, na zuurstof, het tweede meest voorkomende element in de aardkorst. De biogene vorm (biologische oorsprong) van silica (siliciumoxide, ook wel opaal) is, na calciumcarbonaat, het tweede meest voorkomende type mineraal dat wordt gevormd door organismen. Als zodanig is de wereldwijde silicium (Si) cyclus een essentiële component van systeem Aarde en speelt deze bijvoorbeeld een cruciale rol bij het handhaven van de klimaatstabiliteit op geologische tijdschalen. De wereldwijde oceanische Si-cyclus is, op geologische tijdschalen, voornamelijk geëvolueerd tot de hedendaagse cyclus vanwege het verschijnen van biogene silica, en dan met name de opkomst van diatomeeën in de late Krijtperiode.

Diatomeeën (ook wel kiezelwieren genoemd) zijn eencellige microalgen die wijdverspreid in het oppervlaktewater leven. Ze worden in het sediment vertegenwoordigd door hun kiezelhoudende (siliciumdioxide; $\mathrm{SiO}_{2}$ ) exoskeletten. Hoewel de exoskeletten (behuizingen) van diatomeeën de dominante vorm van biogene silica zijn, worden silicastructuren ook gemaakt door verschillende andere aquatische soorten zoals radiolaria, silicoflaggelaten en sponzen.

Het bestuderen van de chemische kenmerken van biogeen fossiel materiaal is van groot belang voor het verschaffen van informatie over het klimaat van vroeger. Dit komt omdat het biogene materiaal, tijdens de groei, de chemie van het omringende water gaat weerspiegelen. Door methodes (genaamd paleoproxies) te ontwikkelen om fossiel materiaal met verschillende leeftijden te analyseren, kunnen we de veranderende waterchemie (en dus het veranderende klimaat) door de tijd heen bestuderen. De laatste decennia krijgt de chemische samenstelling van fossiel biogene silica steeds meer aandacht. Deze toegenomen interesse is deels te wijten aan de schaarste of volledige afwezigheid van carbonaten, een traditioneel doelwitmateriaal voor paleoproxyonderzoek, in delen van het sedimentaire archief (vooral in regio's met een hoge breedtegraad). Gelukkig domineren silica microfossielen, in het bijzonder de overblijfselen van diatomeeën, vaak deze sedimentaire archieven. Vanwege hun relatief kleine omvang (micrometerschaal), houdt de toegenomen interesse ook verband met de voortdurende ontwikkeling van analytische technieken met hoge resolutie zoals de Nanometer Secondary Ion Mass Spectrometer (NanoSIMS).

\section{Overzicht van dit proefschrift}

Het onderzoek in dit proefschrift heeft als doel hoge-resolutie chemische beeldvormingstechnieken toe te passen in een poging om bestaande paleoproxies op basis van biogene silica beter te begrijpen en te verbeteren. We hebben ons vooral gericht op het gebruik van de NanoSIMS, waarmee we chemische kaarten op nanometerschaal kunnen maken. We hebben ook andere complementaire hoge-resolutietechnieken gebruikt, zoals de SIMS en Raman microspectroscopie. 
In hoofdstuk 2 van dit proefschrift bestuderen we fossiele behuizingen van diatomeeën om het oorspronkelijke ijzer(Fe)gehalte, biologisch ingebouwd in het exoskelet tijdens diatomeeëngroei, te onderscheiden van secundaire Fe afkomstig van monsterverontreiniging en diagenese (postmortem fysische en chemische veranderingen die een sediment ondergaat nadat het is afgezet). De $\mathrm{Fe} / \mathrm{Si}$-proxy heeft het potentieel om informatie te verschaffen over de beschikbaarheid van Fe in vroegere kust regio's, en indirect over Si-begraving en $\mathrm{CO}_{2}$-opname door oceanen. Vraagtekens omtrent de mogelijke invloed van postmortale diagenese op de monsters belemmerden echter de ontwikkeling van deze methode. Door de nanoSIMS-techniek toe te passen, konden wij de verdeling van $\mathrm{Fe}$ en $\mathrm{Al}$ (Aluminium; een indicatie voor diagenese) in grondig schoongemaakte fossiele exoskeletten bestuderen. Ondanks de toepassing van een gevestigde reinigingstechniek, hebben we de aanwezigheid van $\mathrm{Al}$-concentraties waargenomen die co-lokaliseerden met sterkere Fe-signalen. We hebben echter ook gevonden dat de binnenkant van de behuizing over het algemeen lagere $\mathrm{Al} / \mathrm{Si}$-waarden had, wat duidt op verminderde post-mortem diagenetische invloeden. Op basis van deze resultaten werden monstervoorbereiding en meetprotocollen voor Fe/ Si-bepalingen met SIMS aangepast door (i) het toevoegen van een extra reinigingsstap en (ii) het richten op de binnenkant van de behuizingen. Deze aanpassingen verlaagden de $\mathrm{Al} / \mathrm{Si}$ en $\mathrm{Fe} / \mathrm{Si}$ ratio's van de gemeten monsters aanzienlijk, waardoor de zorgen rond diagenese worden beperkt en derhalve moeten resulteren in een robuustere paleoproxy.

In hoofdstuk 3 willen we de aanwezigheid en verdeling van organische signalen in grondig gereinigde fossiele behuizingen van diatomeeën in beeld brengen. De toepassing van $\delta 15 \mathrm{~N}$ metingen in deze behuizingen (een proxy voor het gebruik van nitraat vroegere oppervlaktewateren) gaat ervan uit dat de silicastructuur de interne organische materie beschermt tegen diagenese. De exacte locatie van de organische materie in het exoskelet is echter tot nu toe niet geïdentificeerd. Door het combineren van oppervlaktemetingen met hoge ruimtelijke resolutie (nanoSIMS) en Raman micro-spectroscopie (lagere resolutie maar met grotere sondediepte), konden wij voor het eerst de aanwezigheid en verdeling van de silica-gebonden organische materie in beeld brengen. De nanoSIMS-afbeeldingen onthulden dat de organische signalen over het hele exoskelet aanwezig waren, maar met gelokaliseerde concentraties rond de poriën. Raman-metingen bevestigden de heterogene aanwezigheid van organische stoffen, maar konden de door nanoSIMS waargenomen ruimtelijke patronen niet dupliceren.

Hoofdstuk 4 kijkt naar post-mortem chemische veranderingen in het $\delta^{18} \mathrm{O}$-signaal in verschillende vormen van silica. De $\delta^{18} \mathrm{O}$ van water is onder andere een weerspiegeling van veranderingen in het wereldwijde ijsvolume, evenals lokale veranderingen in verdamping, zoetwaterinput (en dus zoutgehalte) en temperatuur. Zowel biogene amorfe silica (exoskeletten van diatomeeën en spons stekels) en kristallijne silica monsters (kwartskristallen) werden geïncubeerd in, met ${ }^{18} \mathrm{O}$ verrijkt, zeewater gedurende verschillende tijdsintervallen. De nanoSIMS-resultaten laten zien dat, op tijdschalen die vergelijkbaar zijn met die van zinkende diatomeeën in de mariene waterkolom (dagen tot weken), het $\delta^{18} \mathrm{O}$-signaal van de biogene monsters homogeen kan worden verrijkt. Kristallijn silica daarentegen vertoont geen aanwezigheid van ${ }^{18} \mathrm{O}$-verrijking, Dit komt hoogstwaarschijnlijk vanwege een verschil in de structuur, namelijk de afwezigheid van silanol-groepen (Si-OH) in de kristallijne silica zoals waargenomen in de Raman-metingen. Samen bevestigen deze resultaten dat de gehele biogene silicastructuur gevoelig is voor diagenese. De uiteindelijk gemeten $\delta^{18} \mathrm{O}_{\text {diatom }}$ zal daarom, hoogstwaarschijnlijk, een gemengd signaal vertegenwoordigen van de initiële groeifase, het zakken door de waterkolom, het sedimentwatergrensvlak en het sedimentporiewater. 
Hoofdstuk 5 zet ons onderzoek naar secundaire zuurstofuitwisseling voort, maar verschuift de focus naar diagenetische post-mortem processen in het sediment. Nadat onze resultaten uit hoofdstuk 4 hebben aangetoond dat diagenese van het $\delta^{18}$ Odiatom signaal al in de waterkolom voorkomt, rijst de vraag of dit in het sediment zou doorgaan of dat extra processen dit zouden remmen. We hebben twee experimenten opgezet om deze vraag te beantwoorden. In het eerste ${ }^{18} \mathrm{O}$-incubatie-experiment werden pakketjes met verse diatomeeën neergelegd in een diatomeeënklei en op het grensvlak van sediment en water. Uit dit experiment konden we verschillende fasen van diagenese in het sediment vergelijken. NanoSIMS-analyse toonde aan dat de verse en fossielen diatomeeën beide significante ${ }^{18} \mathrm{O}$-verrijking ondergingen. De mate waarin dit gebeurde varieerde; het meest verrijkt waren de behuizingen van verse diatomeeën die op het grensvlak van het sediment werden geplaatst, gevolgd door de verse diatomeeën die in het sediment werden begraven en tenslotte de fossiele diatomeeën. Verder hebben we waargenomen dat concentraties van $\mathrm{Al}$ op de oppervlakte van de fossiele behuizingen, de ${ }^{18} \mathrm{O}$-verrijking van het onderliggende siliciumoxide niet remden. In een tweede experiment hebben we variërende concentraties opgelost $\mathrm{Al}(\mathrm{dAl})$ toegevoegd aan met ${ }^{18} \mathrm{O}$ verrijkt zeewater. De toename van dAl leidde tot een toename van het Al-gehalte van de monsters. De verdeling van het extra $\mathrm{Al}$ bleek homogeen te zijn door de behuizing heen, hetgeen een opnemingsmechanisme suggereert waarbij $\mathrm{Al}$ de $\mathrm{Si}$ in de matrix vervangt. De toevoeging van $\mathrm{Al}$ verlaagde de hoeveelheid ${ }^{18} \mathrm{O}$-verrijking aanzienlijk. Deze resultaten brachten ons ertoe om te veronderstellen dat, hoewel de opname van $\mathrm{Al}$ de uitwisseling van secundaire zuurstof kan vertragen, dit waarschijnlijk tot op zekere hoogte op geologische tijdschalen doorgaat. Dit bevestigt onze conclusie uit hoofdstuk 4 dat het gemeten $\delta^{18} \mathrm{O}$-signaal in fossiele diatomeeën waarschijnlijk een combinatie van signalen weerspiegelt die afkomstig zijn van de groeifase tot poriewateren.

In hoofdstuk 6 geef ik een overzicht van mijn ervaringen met de nanoSIMS en andere hoge resolutie analytische technieken, die ik heb opgedaan tijdens mijn werk met biogene silica. Er is weinig precedent wat betreft de toepassing van de nanoSIMS op biogene silica. In dit hoofdstuk worden de vele verschillende benaderingen beschreven die zijn geprobeerd om de nanoSIMS het beste te gebruiken voor deze monsters. We evalueren zowel de succesvolle als de mislukte pogingen. Dit om toekomstige onderzoekers, die geïnteresseerd zijn in het ontrafelen van de vele mysteries rond de chemie van diatomeeën en andere vormen van biogene silica, te informeren over alle onderzoeksbevindingen en te helpen met toekomstig onderzoek. 


$$
\mathbf{A}
$$




\section{Aknowledgments (Dankwoord)}

$\mathrm{Na}$ de vele uren die ik schrijvend heb doorgebracht in 'Op Zuid', kan ik mij geen betere plek bedenken om met mijn cappuccino te reflecteren op de afgelopen vier-en-een-half jaar. Al met al was het een flinke achtbaan, waarbij veel mensen direct en indirect hebben bijgedragen aan de totstandkoming van dit boekje. Het was enorm belangrijk voor mij om te weten dat ik deze uitdaging niet alleen aanging, maar met de steun van vele mensen. $\mathrm{Nu}$ ga ik een poging wagen om alle bedankjes op papier te krijgen.

Laat ik beginnen met mijn promotoren Jack Middelburg en Lubos Polerecky. Jack, bedankt dat je mij deze kans hebt geboden ondanks mijn toch wel middelmatige cijfers in jouw mastervak. Ik hoop dat ik het vertrouwen dat je in mij stopte heb kunnen terugbetalen. Je gaf mij veel vrijheid om een eigen koers te kiezen met dit onderwerp, vandaar waarschijnlijk ook dat er weinig vermeldingen naar $\mathrm{pH}$ zijn in mijn proefschrift, maar dat ik wel een aantal mooie reisjes heb kunnen maken. Ik had in ieder geval geen andere begeleider willen hebben. Jouw encyclopedische brein heeft me keer op keer verbaasd. Zonder die gave had ik menig obscuur artikel uit de jaren ' 80 gemist. Maar ook de gemakkelijke overgang in onze gesprekken, van zwaar wetenschappelijk naar herinneringen uit de studententijd of de huidige geopolitieke situatie, had ik niet willen missen.

Lubos, thank you for providing some well needed realism against my and Jack's (over)optimism. I may not always have been happy with your very thorough feedback but almost every time I had to (grudgingly) admit that you made some very valid points. I sincerely believe that the quality of this thesis received an enormous boost thanks to your input. Also, I would like to apologize to you and your family for the workload I provided you in the final months. It was quite a sprint to the finish which was only possibly because you and Jack were able to correct my manuscripts at breathtaking speeds. Despite the stress this may have provided to you and Jack both, I hope you will look back on this project with fondness.

Ook al had ik officieel maar twee begeleiders, is er toch zeker weten nog een derde begeleider die ik wil bedanken. Michiel, ik durf de uren die wij samen in het pittoreske nanoSIMS lab hebben doorgebracht niet eens te tellen. Wel weet ik dat, zonder jouw gezelschap, ik niet had kunnen garanderen dat de nanoSIMS niet wat klappen had gekregen. Op de moeilijke momenten dat de nanoSIMS niet mee wilde werken hebben we er toch het beste van gemaakt. Vooral de kerstmuziek karaoke zal me nog lang heugen, maar ook jouw geduld en begrip tijdens de wat stroevere periodes in mijn $\mathrm{PhD}$ ben ik erg dankbaar voor. Succes met de nanoSIMS en mocht het even tegenzitten, zet de vooral Mariah even vol volume aan, dan komt het allemaal goed!

Naast mijn begeleiders, zijn er nog twee wetenschappelijke steunpilaren waarvan ik ontzettend blij ben dat ze naast mij staan tijdens de defence. Anne, wat ben ik ontzettend blij dat jij mijn nanoSIMS buddy was en stiekem ook dat jij een jaar eerder bent begonnen. De veerkracht die jij tijdens je $\mathrm{PhD}$, binnen en buiten het wetenschappelijke kader, hebt getoond is ongelofelijk en was 
zeker een inspiratie voor mij. Onze vele koffies samen waren volgens mij voor beide noodzakelijk om onze PhD's door te komen met nog een greintje sanity. Robin, onze vriendschap gaat natuurlijk nog veel verder terug dan het begin van de $\mathrm{PhD}$. Vanaf het tweede jaar van de studie zijn wij vaste (veld)werk maatjes en met jouw kennis en kunde heb jij mij zeker geholpen om beter te presteren. Binnen of buiten het werk, ik kon altijd op jou terugvallen en dat had ik voor geen goud willen missen. Wat ik ook niet had willen missen is onze gezamenlijke congressen, als 'dynamisch duo' liepen we daar rond en ik vind het nog altijd jammer dat onze poging om op een congresdag echt precies hetzelfde te dragen mislukte door een griepje. Ook al gaan we nu opeens naar andere werkgevers en in andere steden wonen, ik hoop dat onze vriendschap nog lang blijft voortbestaan. Joep, eigenlijk ben jij gewoon mijn derde paranimf. Ik vind het erg jammer dat je er niet bij bent op 28 februari, maar heb ontzettend veel respect voor hoe jij bij je principes blijft. Eén van de highlights van mijn $\mathrm{PhD}$ blijft toch wel het Goldschmidt congres in Parijs, het enige congres waar jij, Robin en ik allemaal bij waren. Wat een epische tijden waren dat! Uren door de Parijse nacht lopen, lange wetenschappelijke en filosofische gesprekken voeren en ontzettend veel lachen. Dat congres was een mooie reminder dat we niet moeten stoppen met onze gezamenlijke stedentripjes. Zodra je weer in Europa bent zullen zich hopelijk weer voldoende mogelijkheden aandienen voor wat mooie reisjes met de trein want: Green will always find a way!

Laat ik in de wetenschappelijke context blijven en mijn vele fijne collega's bedanken, beginnend met Team Tremp. Ik had het grote geluk dat ik tijdens mijn PhD meerdere jaren het tweedejaars veldwerk in Tremp mocht begeleiden. En nog meer geluk dat ik dit elk jaar met João en Maarten mocht doen. João bedankt voor jouw mentorschap en vertrouwen, niet alleen in het veld van sedimentaire geologie maar ook daarbuiten. In moeilijke periodes hielp jij mij er overheen te komen en als het goed ging hielp jij mij nog beter te worden. Ook de tutoring die ik van jullie kreeg over de wereld van het songfestival en karaoke, is zeker een verrijking geweest voor mijn leven. Michiel, Chloe and Liesbeth, thanks for the times in Tremp and helping me provide some counterweight to Maarten and João. And remember: a gamba a day keeps the doctor away.

Throughout the four years I have had several office mates. Firstly Niels, Peter and Marie, thanks for surviving the dark caverns of PP9 with me. Anne, Matthias and Olivier, I really enjoyed our time under the improved conditions of the VMA building.

Next I would like to thank the PP9 and VMA lunchbuddies for all the talks about sport, science and everything in between: Wietze, Anne, Niels, Itzel, Nicole, Martijn, Matthias E, Matthias K, Madelina, Nina, Margot, Joost, Luuk, Peter K, Nikki, Thilo, Alejandra, Martin, José, Wim-Joost, Joep, Lauriane, Lex, Caroline en Mariette.

Tijdens mijn PhD heb ik toch ook redelijk wat tijd doorgebracht in de Geo Labs waarbij ik vaak ben bijgestaan door de labtechnici: Thom, John, Tilly, Coen, Helen, Arnold, Klaas, Dineke, Giovanni, Sergei, Bernadette en Katja bedankt! Also I would like to thank everyone at the NIOZ for helping me with the diatom cultures (the babies), measurements and experiment set-ups.

One of the great things about being a NESSC PhD was that I had the opportunity to meet many great people at other institutes in the Netherlands. I really enjoyed organizing and participating in NESSCwork events as well as meeting everyone at conferences and such. Loes, Esmee, Cait, Josh, Linda, Andre, Michiel, Bregje, Margot, Emily, Robin, Anne, Darci, Cindy, Gabriella, Mirjan, Lisa, Bjinse, Nadine, Carolien, I hope I will see all of you at the NESSG alumni events.

My colleagues abroad should not be forgotten as they gave me the opportunity to travel to all these wonderful places. Laetitia, thanks for showing me the ropes of diatom research. Susana, thank you for the chance to visit and work in Saudi Arabia, it was an amazing experience. Other 
amazing experiences and people include the Urbino summer school (special shoutout to Rome gang), NESSC cruise, Red Sea cruise and everyone I met at the conferences. Thanks everyone, I had a blast!

Buiten de universiteit om zijn er nog veel meer mensen die de afgelopen vier jaar mijn verhalen over de $\mathrm{PhD}$ hebben aangehoord en die mij goed hebben kunnen afleiden.

Ten eerste mijn bestuursgenootjes van '14-'15. Ik ben blij dat we nog altijd zo hecht zijn, maar ook dat jullie mij toch elke keer er weer aan herinneren dat ik nog geen publicatie heb. Heel fijn.

Een ander belangrijk bron van vermaak de afgelopen jaren is toch nog steeds mijn ploeg 'Kip onder 'n hoedje' door alle epische vakanties en verdere gezelligheid. Alles hat ein Ende nur die Wurst hat zwei.

Mijn eerste jaar van de $\mathrm{PhD}$ was verder nooit zo leuk geweest als ik niet vele, vele uren had doorgebracht met het coachen van EJD'16. Jens en Floor in het bijzonder heb ik veel tijd mee doorgebracht, fietsend langs het kanaal in elk mogelijk soort weer, en ik ben blij dat ik ze beide nog steeds regelmatig zie. Eigenlijk zijn er nog veel meer (Oud-)Orcanen die de afgelopen jaren leuker hebben gemaakt, eigenlijk teveel om op te noemen want het zijn stuk-voor-stuk super chille mensen - you know who you are! Maar met nog wel even een special mention voor de Kent-geen-grenzen/ DCl8 groep. Verder ook nog iedereen waarmee ik heb gesport in de afgelopen jaren, en dan vooral fietsen, fitness en boulderen, zonder sport had ik allang een burnoutje gehad vermoed ik.

Mijn oud-huisgenoten van J161 zijn ook nog wel het benoemen waard, aangezien mogelijk elk slecht idee dat ik heb gehad in de afgelopen jaren wel een oorsprong heeft bij deze schaduwparanimfen. Desalniettemin hoop ik dat wij doorgaan met onze huisuitjes en dat we nog vele kaarsjes samen mogen aansteken voor Charles en Ronnie.

De volgende categorie is die van (schoon)familie. De schoonfamilie heeft mijn hele $\mathrm{PhD}$ meegekregen en ik vond het fijn dat ik me zo op mijn gemak voelde bij jullie dat het nooit een probleem was als ik me even afsloot van de gezelligheden om nog wat te werken. Wie weet kan ik vanaf heden vaker meedoen met de spelletjes!

Voor mijn eigen familie is de $\mathrm{PhD}$ maar een klein deel van wie ik ben, maar ze weten zeker hoe belangrijk dat deel voor me is geweest. Ondanks dat het soms moeilijk was om uit te leggen wat ik nou precies deed, heb ik nooit gedacht dat ik mijn verhaal niet kwijt kon. Pap, speciaal voor jou heb ik onderzoek gedaan naar monsters uit het Pleistoceen. Mam, bedankt dat je lang geleden in Beijing dat overzichtje hebt gemaakt met mogelijke studies. Ook al had je toen misschien wat twijfels omdat ik niet verder keek dan de A van Aardwetenschappen, ik denk dat we ons er nu wel bij kunnen neerleggen dat het geen slechte keuze was. Zonder jullie als ouders was ik in ieder geval nooit zo ver gekomen. Mae, bedankt voor jouw (illustratieve) bijdrages aan mijn boekje! Ik hoop dat je weet hoe trots ik op jou en jouw artistieke kunsten ben. Joyce, ook al zien we elkaar helaas wat minder dan vroeger, ik vind het altijd fijn om bij jullie thuis te zijn. Het plezier dat jij en Gerben samen hebben, ook als gezin met Evi en Luna, is echt een inspiratie. Opa en Oma Stubbe, ik hoop dat jullie door dit boekje en de verdediging een beetje een idee krijgen van wat ik de afgelopen jaren allemaal heb uitgespookt, naast kaartjes sturen uit verre oorden.

Als laatste kom ik bij de persoon die mijn promotie van heel dichtbij heeft meegemaakt. Annemiek, ik denk eigenlijk dat ik het niet eens in woorden kan omzetten hoe belangrijk jouw bijdrage is geweest. Je hebt me op zoveel manieren geholpen, daar kan ik bijna een heel extra proefschrift over schrijven. Alleen wordt daar uiteindelijk niemand blij van, dus laat ik het op een manier samenvatting die we beide kunnen waarderen: Annemiek bedankt voor alles! 


\section{Curriculum Vitae}

Shaun Akse was born on the 11th of January 1991 in Delft. While growing up, he spent quite some time abroad, living for several years in Thailand and China. This affinity with travel, together with his interest in geography and the beta-sciences, led him to study Earth Sciences at Utrecht University. Here he quickly identified his interest in the changing climates of the past. This interest was further peaked while following the Master Earth, Life and Climate from which he graduated in

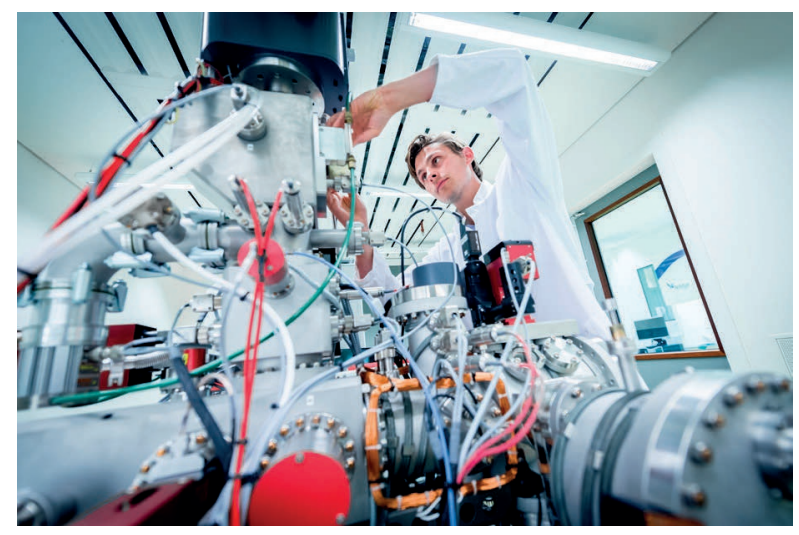
2014. However, he was also active outside the world of academia at the student rowing society A.U.S.R. Orca. This eventually accumulated in him choosing a gap year after receiving his Master's diploma, in the form of a full-time board year. During this year, he came into contact with Prof. Dr. Jack Middelburg at Utrecht University, who presented him with the opportunity of tackling a very promising and exciting Ph.D. project. This pioneering study aimed to reconstruct surface ocean $\mathrm{pH}$ in diatom frustules using the new state-of-the-art NanoSIMS facilities at the Utrecht laboratories. This project started in September 2015 and included expeditions on the Mediterranean and the Red Sea as well as international collaborations with the University of Edinburgh and the King Abdullah University for Science and Technology. During the research, it became clear that the NanoSIMS facilities were better suited for proxy validation than for proxy development. This realization eventually resulted in the chapters presented in this thesis. After finishing the research in the fall of 2019, Shaun decided to pursue a career outside academia and focused his efforts on contributing to a more sustainable world. 


Utrecht University

Faculty of Geosciences

Department of Earth Sciences 